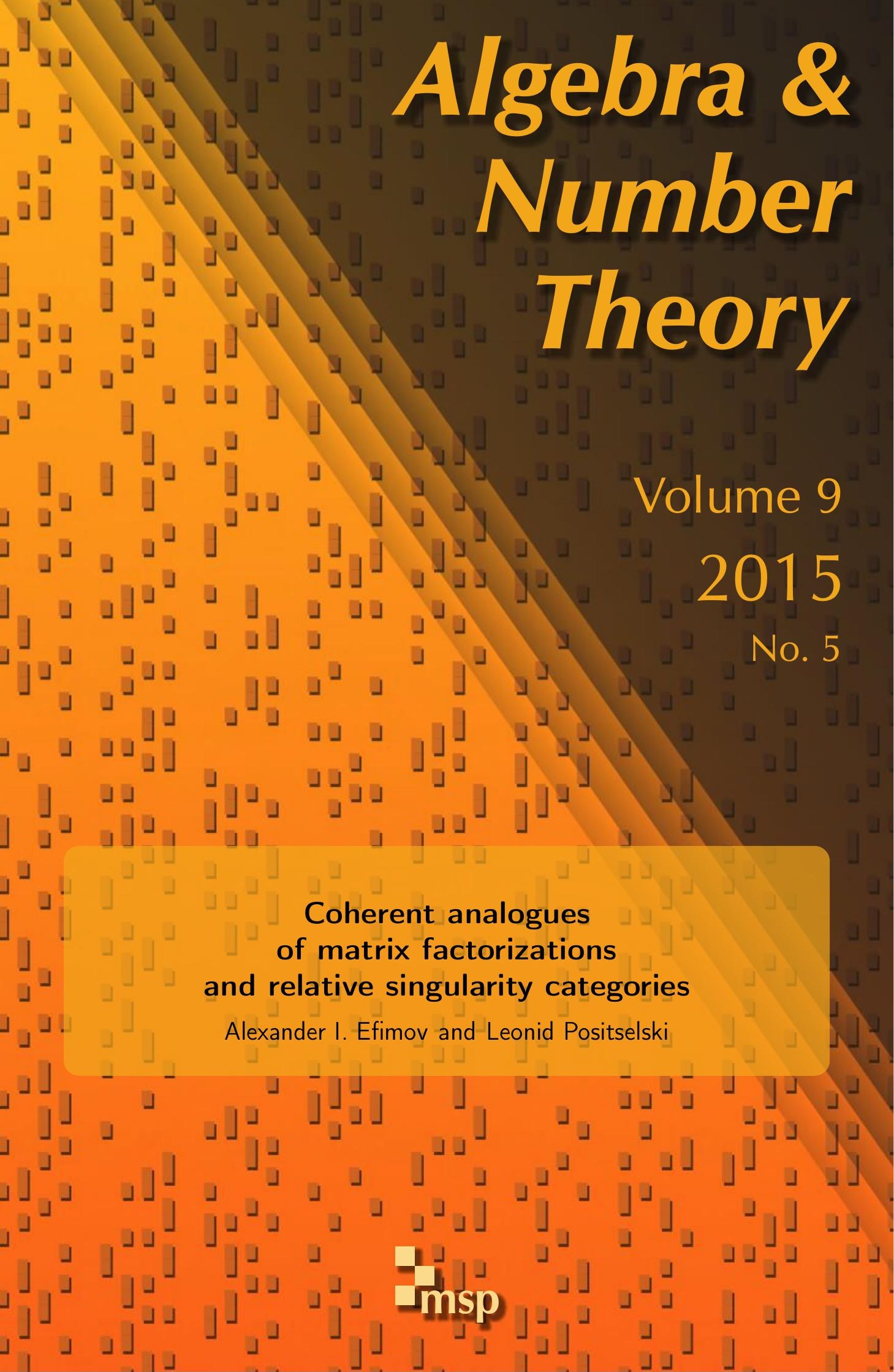




\title{
Coherent analogues of matrix factorizations and relative singularity categories
}

\author{
Alexander I. Efimov and Leonid Positselski
}

We define the triangulated category of relative singularities of a closed subscheme in a scheme. When the closed subscheme is a Cartier divisor, we consider matrix factorizations of the related section of a line bundle, and their analogues with locally free sheaves replaced by coherent ones. The appropriate exotic derived category of coherent matrix factorizations is then identified with the triangulated category of relative singularities, while the similar exotic derived category of locally free matrix factorizations is its full subcategory. The latter category is identified with the kernel of the direct image functor corresponding to the closed embedding of the zero locus and acting between the conventional (absolute) triangulated categories of singularities. Similar results are obtained for matrix factorizations of infinite rank; and two different "large" versions of the triangulated category of relative singularities, corresponding to the approaches of Orlov and Krause, are identified in the case of a Cartier divisor. A version of the Thomason-Trobaugh-Neeman localization theorem is proven for coherent matrix factorizations and disproven for locally free matrix factorizations of finite rank. Contravariant (coherent) and covariant (quasicoherent) versions of the Serre-Grothendieck duality theorems for matrix factorizations are established, and pull-backs and push-forwards of matrix factorizations are discussed at length. A number of general results about derived categories of the second kind for curved differential graded modules (CDG-modules) over quasicoherent CDGalgebras are proven on the way. Hochschild (co)homology of matrix factorization categories are discussed in an appendix.

Introduction

1. Exotic derived categories of quasicoherent CDG-modules

1.1. CDG-rings and CDG-modules

1.2. Quasicoherent CDG-algebras

MSC2010: primary 14F05; secondary 13D09, 16G99.

Keywords: matrix factorizations, relative singularities of Cartier divisors, triangulated categories of singularities, derived categories of the second kind, coderived categories, direct and inverse images, covariant Serre-Grothendieck duality, localization theory, 
1.3. Derived categories of the second kind 1167

1.4. Finite flat dimension theorem 1169

1.5. Fully faithful embedding 1173

1.6. Finite homological dimension theorem 1176

$\begin{array}{ll}\text { 1.7. Gorenstein case } & 1181\end{array}$

1.8. Pull-backs and push-forwards 1182

1.9. Morphisms of finite flat dimension $\quad 1186$

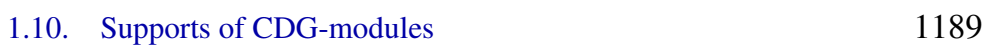

2. Triangulated categories of relative singularities 1192

2.1. Relative singularity category 1192

2.2. Matrix factorizations 1194

2.3. Exotic derived categories of matrix factorizations 1195

2.4. Regular and Gorenstein scheme cases 1196

2.5. Serre-Grothendieck duality 1198

2.6. w-flat matrix factorizations 1205

2.7. Main theorem 1206

2.8. Infinite matrix factorizations $\quad 1214$

2.9. Stable derived category 1218

2.10. Relative stable derived category 1222

3. Supports, pull-backs, and push-forwards 1225

3.1. Supports 1225

3.2. Locality of local freeness 1228

3.3. Nonlocalization of local freeness 1233

3.4. Pull-backs and push-forwards in singularity categories $\quad 1236$

3.5. Push-forwards of matrix factorizations 1238

3.6. Push-forwards for morphisms of finite flat dimension $\quad 1240$

3.7. Duality and push-forwards 1244

$\begin{array}{ll}\text { 3.8. Duality and pull-backs } & 1247\end{array}$

Appendix A. Quasicoherent graded modules 1249

$\begin{array}{ll}\text { A.1. Flat quasicoherent sheaves } & 1249\end{array}$

A.2. Locally projective quasicoherent graded modules 1250

A.3. Injective quasicoherent graded modules 1250

Appendix B. Hochschild (co)homology of matrix factorizations 1253

B.1. Locally free matrix factorizations of finite rank 1253

B.2. Coherent matrix factorizations 1264

$\begin{array}{ll}\text { Acknowledgments } & 1289\end{array}$

$\begin{array}{ll}\text { References } & 1289\end{array}$ 


\section{Introduction}

A matrix factorization of an element $w$ in a commutative ring $R$ is a pair of square matrices $(\Phi, \Psi)$ of the same size, with entries from $R$, such that both the products $\Phi \Psi$ and $\Psi \Phi$ are equal to $w$ times the identity matrix. In the coordinate-free language, a matrix factorization is a pair of finitely generated free $R$-modules $M^{0}$ and $M^{1}$ together with $R$-module homomorphisms $M^{0} \rightarrow M^{1}$ and $M^{1} \rightarrow M^{0}$ such that both the compositions $M^{0} \rightarrow M^{1} \rightarrow M^{0}$ and $M^{1} \rightarrow M^{0} \rightarrow M^{1}$ are equal to the multiplication with $w$. Matrix factorizations were introduced by Eisenbud [1980] and used by Buchweitz [1986] for the study of the maximal Cohen-Macaulay modules over hypersurface local rings.

Another name for this notion is "D-branes in the Landau-Ginzburg B model" (as suggested by Kontsevich) [Kapustin and Li 2003]; in this context, the element $w$ is called the potential. One generalizes the above definition, replacing free modules with projective modules [Kapustin and Li 2003; Orlov 2004], with locally free sheaves [Orlov 2012], and finally with coherent sheaves [Lin and Pomerleano 2013]. The importance of the latter generalization is emphasized in the present paper.

Being particular cases of curved DG-modules over a curved DG-ring [Kapustin and Li 2003; Positselski 2011b], matrix factorizations form a DG-category. So one can consider the corresponding category of closed degree-zero morphisms up to chain homotopy, which is a triangulated category. Generally speaking, however, the homotopy category is "too big" for most purposes, and one would like to pass from it to an appropriately defined derived category. One can use the homotopy category in lieu of the derived one when dealing with projective modules [Kapustin and Li 2003; Orlov 2004]; for locally free matrix factorizations over a nonaffine scheme, there is an option of working with the quotient category of the homotopy category by the locally contractible objects [Polishchuk and Vaintrob 2011, Definition 3.13]. When dealing with coherent (analogues of) matrix factorizations, having some kind of derived category construction is apparently unavoidable.

The relevant concept of a derived category is that of the derived category of the second kind, as developed in [Positselski 2010; 2011b]. There are several versions of this notion; the appropriate one for quasicoherent sheaves is called the coderived category and for coherent sheaves it is the absolute derived category. The absolute derived category of locally free matrix factorizations was studied in [Orlov 2012]; for coherent matrix factorizations over a smooth variety, it was considered in [Lin and Pomerleano 2013]. These two absolute derived categories are equivalent for regular schemes, but can be different otherwise (as we show with an explicit counterexample).

The triangulated category of singularities of a Noetherian scheme was defined by D. Orlov [2004] as the quotient category of the bounded derived category of 
coherent sheaves by its full triangulated subcategory of perfect complexes, i.e., the objects locally presentable as finite complexes of locally free sheaves. This triangulated category vanishes if and only if the Noetherian scheme is regular. It was shown in [Orlov 2004, Theorem 3.9], under mild assumptions on an affine regular Noetherian scheme $X$ and a potential (regular function) $w$ on it, that the homotopy category of locally free matrix factorizations of $w$ over $X$ is equivalent to the triangulated category of singularities of the zero locus $X_{0}$ of $w$ in $X$.

Orlov [2012] showed that the affineness assumption on $X$ can be dropped in this result if one replaces the homotopy category of locally free matrix factorizations with their absolute derived category. He also considers the general case of a nonaffine singular scheme $X$, for which he obtains a fully faithful functor from the absolute derived category of locally free matrix factorizations over $X$ to the triangulated category of singularities of $X_{0}$. The problem of studying the difference between these two triangulated categories was posed in the introduction to [Orlov 2012].

The first aim of the present paper is to provide an alternative proof of these results of Orlov for regular schemes, an alternative generalization of them to singular schemes, and a more precise version of Orlov's original generalization. We replace the triangulated category at the source of Orlov's fully faithful functor by a "larger" category (containing the original one) and the triangulated category at the target by a "smaller" category (a quotient of the original one), thereby transforming this functor into an equivalence of triangulated categories. We also describe the image of Orlov's fully faithful functor as the kernel of a certain other triangulated functor.

More precisely, we show that the absolute derived category of coherent matrix factorizations of $w$ over $X$ is equivalent to what we call the triangulated category of singularities of $X_{0}$ relative to $X$. The latter category is a certain quotient category of the triangulated category of singularities of $X_{0}$; it measures, roughly speaking, how much worse are the singularities of $X_{0}$ compared to those of $X$. As to the image of Orlov's fully faithful embedding, it consists precisely of those objects of the conventional (absolute) triangulated category of singularities of $X_{0}$ whose direct images vanish in the triangulated category of singularities of $X$.

The paper consists of three sections and two appendices. In Section 1, we prove three rather general technical assertions about derived categories of the second kind for curved differential graded modules (CDG-modules) over a quasicoherent CDG-algebra with a restriction on the homological dimension. One of them, claiming that certain embeddings of DG-categories of CDG-modules induce equivalences of the derived categories of the second kind, is a generalization of [Polishchuk and Positselski 2012, Theorem 3.2] based on a modification of the same argument, originally introduced for the proof of [Positselski 2010, Theorem 7.2.2].

The idea of the proof of the other assertion, according to which certain natural functors between derived categories of the second kind are fully faithful, is new. 
The third technical assertion explains when the coderived category coincides with the absolute derived category of the same class of CDG-modules: e.g., for the locally projective CDG-modules this is true.

A version of (the former two of) these results is used in Section 2 to extend Orlov's cokernel functor from the absolute derived category of locally free matrix factorizations to the absolute derived category of coherent ones. This extension of the cokernel functor admits a simple construction of a functor in the opposite direction, suggested in [Lin and Pomerleano 2013]. We use these constructions to obtain a new proof of Orlov's theorem, and our own generalization of it to the singular case.

When $X$ is regular, Orlov's and our results amount to the same assertion since the absolute derived categories of locally free and coherent matrix factorizations are equivalent by our Theorem 1.4. When $X$ is singular, the natural functor between these two absolute derived categories is fully faithful by our Proposition 1.5, and Orlov's full-and-faithfulness theorem follows from ours by virtue of an appropriate semiorthogonality property.

We also compare a "large" version of the triangulated category of relative singularities with the coderived category of quasicoherent matrix factorizations, strengthening some results of Polishchuk and Vaintrob [2011]. A "large" version of the absolute triangulated category of singularities, defined by Orlov [2004], is identified with H. Krause's stable derived category [2005] in the case of a divisor in a regular scheme. A similar result is proven in the case of a Cartier divisor in a singular scheme, where we extend Krause's theory by defining the relative stable derived category. For any closed subscheme of finite flat dimension in a separated Noetherian scheme, the relative stable derived category is compactly generated by its full triangulated subcategory equivalent to the triangulated category of relative singularities.

The homotopy categories of unbounded complexes of projective modules over a ring and injective quasicoherent sheaves over a scheme were studied by Jørgensen [2005] and Krause [2005]; subsequently, Iyengar and Krause [2006] constructed an equivalence between these two categories for rings with dualizing complexes. These results were extended to quasicoherent sheaves over schemes by Neeman [2008] and Murfet [2007], who found a way to define a replacement of the homotopy category of (nonexistent) projective sheaves in terms of the flat ones. The equivalence between these two categories is a covariant version of Serre-Grothendieck duality [Hartshorne 1966]. It is also very similar to the derived comodule-contramodule correspondence theory, developed by the second author [Positselski 2010; 2011b].

Serre-Grothendieck duality for matrix factorizations in the situation of a smooth variety $X$ (and an isolated singularity of $X_{0}$ ) was studied in [Murfet 2013]. In this paper we extend the duality to matrix factorizations over much more general schemes $X$, constructing an equivalence between two "large" exotic derived categories, namely, the coderived category of flat (or locally free) matrix factorizations 
of possibly infinite rank and the coderived category of quasicoherent matrix factorizations. Unless $X$ is Gorenstein, this equivalence is not provided by the natural functor induced by the embedding of DG-categories, but rather differs from it in that the tensor product with the dualizing complex has to be taken along the way. A contravariant Serre duality in the form of an auto-antiequivalence of the absolute derived category of coherent matrix factorizations is also obtained.

There was some attention paid to pull-backs and push-forwards of matrix factorizations recently [Polishchuk and Vaintrob 2011; 2014; Dyckerhoff and Murfet 2013]. In Section 3, we approach this topic with our techniques, constructing the push-forwards of locally free matrix factorizations of infinite rank for any morphism of finite flat dimension between schemes of finite Krull dimension, and the pushforwards of locally free matrix factorizations of finite rank for any such morphism for which the induced morphism of the zero loci of $w$ is proper. At the price of having to adjoin the images of idempotent endomorphisms, the preservation of finite rank under push-forwards is proven assuming only the support of the matrix factorization [Polishchuk and Vaintrob 2011] to be proper over the base.

Push-forwards of quasicoherent matrix factorizations are well-defined for any morphism of Noetherian schemes, and push-forwards of coherent matrix factorizations exist under properness assumptions similar to the above. A general study of category-theoretic and set-theoretic supports of quasicoherent and coherent CDG-modules is undertaken in this paper in order to obtain an independent proof of the preservation of coherence under the push-forwards not based on the passage to the triangulated categories of singularities.

The compatibility with pull-backs and push-forwards is an organic part of Serre-Grothendieck duality theory. The contravariant duality agrees with pushforwards of coherent sheaves (or matrix factorizations) with respect to proper morphisms [Hartshorne 1966], while the covariant duality transforms the conventional inverse image of flat matrix factorizations into the extraordinary inverse image of quasicoherent ones [Positselski 2012]. We use the latter result in order to construct the extraordinary inverse image functor of Hartshorne and Deligne, which is denoted by $f^{!}$in [Hartshorne 1966] and which we denote by $f^{+}$, in the case of quasicoherent matrix factorizations.

Appendix A contains proofs of some basic facts about flat, locally projective, and injective quasicoherent graded modules which are occasionally used in the main body of the paper. Appendix B can be viewed as a complement to the paper [Polishchuk and Positselski 2012]. While Section B.1 contains some variations of and improvements on the results about Hochschild (co)homology of (C)DG-categories and (locally free) matrix factorizations in [loc. cit.], Section B.2 presents an alternative approach to the Hochschild (co)homology of coherent matrix factorizations based on the techniques developed in the main body of this paper. 


\section{Exotic derived categories of quasicoherent CDG-modules}

1.1. CDG-rings and $C D G$-modules. A $C D G$-ring (curved differential graded ring) $B=(B, d, h)$ is defined as a graded ring $B=\bigoplus_{i \in \mathbb{Z}} B^{i}$ endowed with an odd derivation $d: B \rightarrow B$ of degree 1 and an element $h \in B^{2}$ such that $d^{2}(b)=$ $[h, b]$ for all $b \in B$ and $d(h)=0$. So one should have $d: B^{i} \rightarrow B^{i+1}$ and $d(a b)=d(a) b+(-1)^{|a|} a d(b)$; the brackets [-,-] denote the supercommutator $[a, b]=a b-(-1)^{|a||b|} b a$. The element $h$ is called the curvature element.

A morphism of CDG-rings $B \rightarrow A$ is a pair $(f, a)$, with a morphism of graded rings $f: B \rightarrow A$ and an element $a \in A^{1}$ such that $f\left(d_{B} b\right)=d_{A} f(b)+[a, f(b)]$ for all $b \in B$ and $f\left(h_{B}\right)=h_{A}+d_{A} a+a^{2}$. The composition of morphisms of CDG-rings is defined by the obvious rule $(f, a) \circ(g, b)=(f \circ g, a+f(b))$. The element $a$ is called the change-of-connection element. A discussion of the origins of these definitions can be found in the paper [Positsel'skiı 1993], where the above terminology first appeared (see also an earlier paper [Getzler and Jones 1990], where the motivation was entirely different).

A left $C D G$-module $M=\left(M, d_{M}\right)$ over a CDG-ring $B$ is a graded $B$-module endowed with an odd derivation $d_{M}: M \rightarrow M$ compatible with the derivation $d$ on $B$ such that $d_{M}^{2}(m)=h m$ for all $m \in M$. Given a morphism of CDG-rings $(f, a): B \rightarrow A$ and a CDG-module $(M, d)$ over $A$, the CDG-module $\left(M, d^{\prime}\right)$ over $B$ is defined by the rule $d^{\prime}(m)=d(m)+a m$.

Given graded left $B$-modules $M$ and $N$, homogeneous $B$-module morphisms $f: M \rightarrow N$ of degree $n$ are defined as homogeneous maps supercommuting with the action of $B$; i.e., $f(b m)=(-1)^{n|b|} b f(m)$. When $M$ and $N$ are CDG-modules, the homogeneous $B$-module morphisms $M \rightarrow N$ form a complex of abelian groups with the differential $d(f)(m)=d(f(m))-(-1)^{|f|} f(d(m))$. The curvature-related terms cancel out in the computation of the square of this differential, so one has $d^{2}(f)=0$. Therefore, left CDG-modules over $B$ form a DG-category.

Two aspects of the above definitions are worth pointing out. First, the CDG-rings or modules have no cohomology modules, as their differentials do not square to zero. Second, given a CDG-ring $B$, there is no natural way to define a CDG-module structure on the free graded $B$-module $B$ (though $B$ is naturally a CDG-bimodule over itself, in the appropriate sense).

We refer the reader to [Positselski 2011b, Section 3.1] or [Positselski 2010, Sections 0.4.3-0.4.5] for more detailed discussions of the above notions. We will not need to consider any gradings different from $\mathbb{Z}$-gradings in this paper, though all the general results will be equally applicable in the $\Gamma$-graded situation in the sense of [Polishchuk and Positselski 2012, Section 1.1].

1.2. Quasicoherent CDG-algebras. Throughout this paper, unless specified otherwise, $X$ is a separated Noetherian scheme with enough vector bundles; in other 
words, it is assumed that every coherent sheaf on $X$ is the quotient sheaf of a locally free sheaf of finite rank. Note that the class of all schemes satisfying these conditions is closed under the passages to open and closed subschemes [Orlov 2004, Section 1.2] and contains all regular separated Noetherian schemes [Hartshorne 1977, Exercise III.6.8].

Recall the definition of a quasicoherent CDG-algebra from [Positselski 2011b, Appendix B]. A quasicoherent CDG-algebra $\mathcal{B}$ over $X$ is a graded quasicoherent $\mathcal{O}_{X}$-algebra such that for each affine open subscheme $U \subset X$, the graded ring $\mathcal{B}(U)$ is endowed with a structure of CDG-ring, i.e., a (not necessarily $\mathcal{O}_{X}$-linear) odd derivation $d: \mathcal{B}(U) \rightarrow \mathcal{B}(U)$ of degree 1 and an element $h \in \mathcal{B}^{2}(U)$. For each pair of embedded affine open subschemes $U \subset V \subset X$, an element $a_{U V} \in \mathcal{B}^{1}(U)$ is fixed such that the restriction morphism $\mathcal{B}(V) \rightarrow \mathcal{B}(U)$ together with the element $a_{U V}$ form a morphism of CDG-rings. The obvious compatibility condition is imposed for triples of embedded affine open subschemes $U \subset V \subset W \subset X$.

A quasicoherent left $C D G$-module $\mathcal{M}$ over $\mathcal{B}$ is an $\mathcal{O}_{X}$-quasicoherent (or, equivalently, $\mathcal{B}$-quasicoherent) sheaf of graded left modules over $\mathcal{B}$ together with a family of differentials $d: \mathcal{M}(U) \rightarrow \mathcal{M}(U)$ defined for all affine open subschemes $U \subset X$ such that $\mathcal{M}(U)$ is a CDG-module over $\mathcal{B}(U)$ and the appropriate compatibility condition holds with respect to the restriction morphisms of CDG-rings $\mathcal{B}(V) \rightarrow \mathcal{B}(U)$. Specifically, for a quasicoherent left CDG-module $\mathcal{M}$, one should have

$$
\left.d(s)\right|_{U}=d\left(\left.s\right|_{U}\right)+\left.a_{U V} s\right|_{U} \quad \text { for any } s \in \mathcal{M}(V) .
$$

Quasicoherent left CDG-modules over a quasicoherent CDG-algebra $\mathcal{B}$ form a DG-category [Positselski 2011b]. The complex of morphisms between CDG-modules $\mathcal{N}$ and $\mathcal{M}$ is the graded abelian group of homogeneous $\mathcal{B}$-module morphisms $f: \mathcal{N} \rightarrow \mathcal{M}$ with the differential $d(f)$ defined locally as the supercommutator of $f$ with the differentials in $\mathcal{N}(U)$ and $\mathcal{M}(U)$. We denote this DG-category by $\mathcal{B}$-qcoh.

We will call a quasicoherent graded algebra $\mathcal{B}$ over $X$ Noetherian if the graded ring $\mathcal{B}(U)$ is left Noetherian for any affine open subscheme $U \subset X$. Equivalently, $\mathcal{B}$ is Noetherian if the abelian category of quasicoherent graded left $\mathcal{B}$-modules is a locally Noetherian Grothendieck category. In this case, the full DG-subcategory in $\mathcal{B}$-qcoh formed by $\mathrm{CDG}$-modules whose underlying graded $\mathcal{B}$-modules are coherent (i.e., finitely generated over $\mathcal{B}$ ) is denoted by $\mathcal{B}$-coh.

Given a quasicoherent graded left $\mathcal{B}$-module $\mathcal{M}$ and a quasicoherent graded right $\mathcal{B}$-module $\mathcal{N}$, one can define their tensor product $\mathcal{N} \otimes_{\mathcal{B}} \mathcal{M}$, which is a quasicoherent graded $\mathcal{O}_{X}$-module. A quasicoherent graded left $\mathcal{B}$-module $\mathcal{M}$ is called flat if the functor $-\otimes_{\mathcal{B}} \mathcal{M}$ is exact on the abelian category of quasicoherent graded right $\mathcal{B}$-modules. Equivalently, $\mathcal{M}$ is flat if the graded left $\mathcal{B}(U)$-module $\mathcal{M}(U)$ is flat for any affine open subscheme $U \subset X$. The flat dimension of a quasicoherent graded module $\mathcal{M}$ is the minimal length of its flat left resolution. 
The full DG-subcategory in $\mathcal{B}$-qcoh formed by CDG-modules whose underlying graded $\mathcal{B}$-modules are flat is denoted by $\mathcal{B}$-qcoh ${ }_{\mathrm{fl}}$, and the full subcategory formed by CDG-modules whose underlying graded $\mathcal{B}$-modules have finite flat dimension is denoted by $\mathcal{B}$-qcoh ffd $_{\text {. The similarly defined DG-categories of coherent CDG-modules }}$ are denoted by $\mathcal{B}$ - coh $_{\mathrm{fl}}$ and $\mathcal{B}$ - coh $_{\mathrm{ffd}}$.

All the above DG-categories of quasicoherent CDG-modules (and the similar ones defined below in this paper) admit shifts and twists, and, in particular, cones. It follows that their homotopy categories $H^{0}(\mathcal{B}$-qcoh $), H^{0}(\mathcal{B}$-qcoh fl $), H^{0}(\mathcal{B}$-coh $)$, etc. are triangulated. Besides, to any finite complex (of objects and closed morphisms) in one of these DG-categories, one can assign its total object, which is an object of (i.e., a CDG-module belonging to) the same DG-category [Positselski 2011b, Section 1.2].

The DG-categories $\mathcal{B}$-qcoh and $\mathcal{B}$-qcoh ${ }_{\mathrm{fl}}$ also admit infinite direct sums. Hence in these two DG-categories one can totalize even an unbounded complex by taking infinite direct sums along the diagonals.

The DG-category $\mathcal{B}$-qcoh also admits infinite products (which one can obtain using the coherator construction from [Thomason and Trobaugh 1990, Section B.14]), but these are not well-behaved (neither exact nor local), so we will not use them.

\subsection{Derived categories of the second kind. The nonexistence of the cohomology} groups for curved structures stands in the way of the conventional definition of the derived category of CDG-modules, which therefore does not seem to make sense. The suitable class of constructions of derived categories for CDG-modules is that of the derived categories of the second kind [Positselski 2010; 2011b].

Let $\mathcal{B}$ be a quasicoherent CDG-algebra over $X$; assume that the quasicoherent graded algebra $\mathcal{B}$ is Noetherian. Then a coherent $\mathrm{CDG}$-module over $\mathcal{B}$ is called absolutely acyclic if it belongs to the minimal thick subcategory of the homotopy category of coherent CDG-modules $H^{0}(\mathcal{B}$-coh) containing the total CDG-modules of all the short exact sequences of coherent $\mathrm{CDG}$-modules over $\mathcal{B}$ (with closed morphisms between them). The quotient category of $H^{0}(\mathcal{B}$-coh) by the thick subcategory of absolutely acyclic CDG-modules is called the absolute derived category of coherent CDG-modules over $\mathcal{B}$ and denoted by $\mathrm{D}^{\text {abs }}(\mathcal{B}$-coh) [Positselski 2011b].

For any quasicoherent $C D G$-algebra $\mathcal{B}$ over $X$, a quasicoherent CDG-module over $\mathcal{B}$ is called coacyclic if it belongs to the minimal triangulated subcategory of the homotopy category of quasicoherent CDG-modules $H^{0}(\mathcal{B}$-qcoh) containing the total CDG-modules of all the short exact sequences of quasicoherent CDG-modules over $\mathcal{B}$ and closed under infinite direct sums. The quotient category of $H^{0}(\mathcal{B}$-coh) by the thick subcategory of coacyclic CDG-modules is called the coderived category of quasicoherent $\mathrm{CDG}$-modules over $\mathcal{B}$ and denoted by $\mathrm{D}^{\mathrm{co}}(\mathcal{B}$-qcoh) [Positselski 2010; 2011b]. 
Given an exact subcategory $E$ in the abelian category of quasicoherent graded left $\mathcal{B}$-modules, one can define the absolute derived category of left $C D G$-modules over $\mathcal{B}$ with the underlying graded $\mathcal{B}$-modules belonging to $\mathrm{E}$ as the quotient category of the corresponding homotopy category by its minimal thick subcategory containing the total CDG-modules of all the exact triples of CDG-modules with the underlying graded $\mathcal{B}$-modules belonging to $\mathrm{E}$. The objects of the latter subcategory are called absolutely acyclic with respect to $\mathrm{E}$ (or with respect to the DG-category of CDG-modules with the underlying graded modules belonging to E) [Polishchuk and Positselski 2012].

In particular, one defines the absolute derived categories $D^{\text {abs }}\left(\mathcal{B}\right.$-coh $\left.{ }_{\mathrm{ffd}}\right)$ and $\mathrm{D}^{\text {abs }}\left(\mathcal{B}\right.$ - coh $\left._{\mathrm{fl}}\right)$ as the quotient categories of the homotopy categories $H^{0}\left(\mathcal{B}\right.$-coh ffd $\left._{\mathrm{fd}}\right)$ and $H^{0}\left(\mathcal{B}\right.$-coh $\left.h_{f l}\right)$ by the thick subcategories of CDG-modules absolutely acyclic with respect to $\mathcal{B}$-coh ffd $_{\text {and }} \mathcal{B}$-coh fl $_{\text {, }}$, respectively.

When the exact subcategory $E$ is closed under infinite direct sums, the thick subcategory of CDG-modules coacyclic with respect to $\mathrm{E}$ is the minimal triangulated subcategory of the homotopy category CDG-modules with the underlying graded modules belonging to $\mathrm{E}$, containing the total CDG-modules of all the exact triples of CDG-modules with the underlying graded modules belonging to $\mathrm{E}$ and closed under infinite direct sums. The quotient category by this thick subcategory is called the coderived category of left $C D G$-modules over $\mathcal{B}$ with the underlying graded modules belonging to E [Positselski 2010; Polishchuk and Positselski 2012].

Thus one defines the coderived category $\mathrm{D}^{\mathrm{co}}\left(\mathcal{B}-q \mathrm{coh}_{\mathrm{fl}}\right)$ as the quotient categories of the homotopy category $H^{0}\left(\mathcal{B}\right.$-qcoh $\left.{ }_{\mathrm{fl}}\right)$ by the thick subcategory of CDG-modules coacyclic with respect to $\mathcal{B}-q c_{\mathrm{fl}}$. A little more care is needed for the definition of the coderived category $\mathrm{D}^{\mathrm{co}}\left(\mathcal{B}\right.$-qcoh $\left.\mathrm{ffd}_{\mathrm{ff}}\right)$ since the class of graded modules of finite flat dimension is not in general closed under infinite direct sums. An object $\mathcal{M} \in H^{0}\left(\mathcal{B}\right.$-qcoh $\left.{ }_{\text {ffd }}\right)$ is said to be coacyclic with respect to $\mathcal{B}$-qcoh ${ }_{\text {ffd }}$ if there exists an integer $d \geq 0$ such that $\mathcal{M}$ is coacyclic with respect to the exact category of quasicoherent CDG-modules of flat dimension at most $d$. The coderived category of quasicoherent CDG-modules of finite flat dimension is, by the definition, the quotient category of $H^{0}\left(\mathcal{B}\right.$-qcoh $\left.{ }_{\text {ffd }}\right)$ by the above-defined thick subcategory of coacyclic CDG-modules [Polishchuk and Positselski 2012, Section 3.2].

Remark 1.3. One may wonder whether coacyclicity (absolute acyclicity) of quasicoherent CDG-modules (of a certain class) is a local notion. One general approach to this kind of problem is to consider the Mayer-Vietoris/Čech exact sequence

$$
0 \longrightarrow \mathcal{M} \longrightarrow \bigoplus_{\alpha} j_{U_{\alpha} *} j_{U_{\alpha}}^{*} \mathcal{M} \longrightarrow \bigoplus_{\alpha<\beta} j_{U_{\alpha} \cap \mathcal{U}_{\beta} *} j_{U_{\alpha} \cap U_{\beta}}^{*} \mathcal{M} \longrightarrow \cdots \longrightarrow 0
$$

for a finite affine open covering $U_{\alpha}$ of $X$. Since the inverse and direct images with respect to affine open embeddings are exact and compatible with direct sums, they 
preserve coacyclicity (absolute acyclicity). Hence if the restrictions of $\mathcal{M}$ to all $U_{\alpha}$ are coacyclic (absolutely acyclic), then so is $\mathcal{M}$ itself.

Alternatively, one can base this kind of argument on the implications of the Noetherianness assumption, rather than the separatedness assumption. For this purpose, one replaces a quasicoherent CDG-module $\mathcal{M}$ with its injective resolution (see Lemma 1.7(b)) before writing down its Čech resolution. In this approach, the covering need not be affine, as injective coacyclic objects are contractible, and direct images preserve contractibility; but it is important that the restrictions to open subschemes should preserve injectivity of quasicoherent graded $\mathcal{B}$-modules (see [Hartshorne 1966, Theorem II.7.18] and Theorem A.3; cf. [Thomason and Trobaugh 1990, Appendix B]).

When one is working with coherent CDG-modules, the Čech sequence argument is to be used in conjunction with Proposition 1.5 below. (Cf. Sections 1.10 and 3.2.)

1.4. Finite flat dimension theorem. The next theorem is our main technical result on which the proofs in Section 2 are based.

Though we generally prefer the coderived categories of (various classes of) infinitely generated CDG-modules over their absolute derived categories, technical considerations sometimes force us to deal with the latter (see Remark 1.5). Therefore, let $D^{\text {abs }}\left(\mathcal{B}\right.$-qcoh $\left.{ }_{\mathrm{fl}}\right)$, $\mathrm{D}^{\mathrm{abs}}\left(\mathcal{B}\right.$-qcoh $\left.{ }_{\mathrm{ffd}}\right)$, and $\mathrm{D}^{\mathrm{abs}}(\mathcal{B}$-qcoh) denote the absolute derived categories of (flat, of finite flat dimension, or arbitrary) quasicoherent CDG-modules over a quasicoherent CDG-algebra $\mathcal{B}$.

Theorem 1.4. (a) For any quasicoherent $C D G$-algebra $\mathcal{B}$ over $X$, the functor

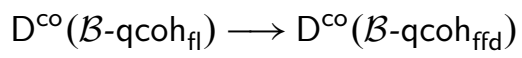

induced by the embedding of DG-categories $\mathcal{B}$-qcoh $\mathrm{fl}_{\mathrm{fl}} \rightarrow \mathcal{B}$-qcoh $\mathrm{ffd}_{\mathrm{ff}}$ is an equivalence of triangulated categories.

(b) For any quasicoherent CDG-algebra $\mathcal{B}$ over $X$, the functor

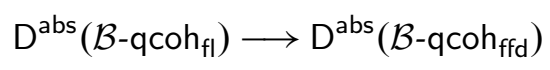

induced by the embedding of DG-categories $\mathcal{B}$ - $\mathrm{qcoh}_{\mathrm{fl}} \rightarrow \mathcal{B}$-qcoh $\mathrm{ffd}_{\mathrm{ff}}$ is an equivalence of triangulated categories.

(c) For any quasicoherent $C D G$-algebra $\mathcal{B}$ over $X$ such that the underlying quasicoherent graded algebra $\mathcal{B}$ is Noetherian, the functor

$$
\mathrm{D}^{\mathrm{abs}}\left(\mathcal{B}-\operatorname{coh}_{\mathrm{fl}}\right) \longrightarrow \mathrm{D}^{\mathrm{abs}}\left(\mathcal{B}-\mathrm{coh}_{\mathrm{ffd}}\right)
$$

induced by the embedding of DG-categories $\mathcal{B}$ - $\operatorname{coh}_{\mathrm{fl}} \rightarrow \mathcal{B}$-coh $\mathrm{chd}_{\mathrm{ff}}$ is an equivalence of triangulated categories. 
Proof. The proof follows that of [Polishchuk and Positselski 2012, Theorem 3.2] (see also [Positselski 2010, Theorem 7.2.2]) with some modifications. We will prove part (a); the proofs of parts (b) and (c) are similar. (Alternatively, parts (b) and (c) can be deduced from Proposition 1.5(a) and (b) below.)

Given an affine open subscheme $U \subset X$ and a graded module $P$ over the graded ring $\mathcal{B}(U)$, one can construct the freely generated CDG-module $G^{+}(P)$ over the CDG-ring $\mathcal{B}(U)$ in the way explained in [Positselski 2011b, proof of Theorem 3.6]. The elements of $G^{+}(P)$ are formal expressions of the form $p+d q$, where $p, q \in P$. Given a quasicoherent graded module $\mathcal{P}$ over $\mathcal{B}$, the CDG-modules $G^{+}(\mathcal{P}(U))$ glue together to form a quasicoherent CDG-module $G^{+}(\mathcal{P})$ over $\mathcal{B}$. For any quasicoherent CDG-module $\mathcal{M}$ over $\mathcal{B}$, there is a bijective correspondence between morphisms of graded $\mathcal{B}$-modules $\mathcal{P} \rightarrow \mathcal{M}$ and closed morphisms of CDG-modules $G^{+}(\mathcal{P}) \rightarrow \mathcal{M}$ over $\mathcal{B}$. There is a natural short exact sequence of quasicoherent graded $\mathcal{B}$-modules $\mathcal{P} \rightarrow G^{+}(\mathcal{P}) \rightarrow \mathcal{P}[-1]$. The quasicoherent CDG-module $G^{+}(\mathcal{P})$ is naturally contractible with the contracting homotopy $t_{\mathcal{P}}$ given by the composition

$$
G^{+}(\mathcal{P}) \longrightarrow \mathcal{P}[-1] \longrightarrow G^{+}(\mathcal{P})[-1]
$$

Due to our assumption on $X$, for any quasicoherent $\mathcal{O}_{X}$-module $\mathcal{K}$ over $X$ there exists a surjective morphism $\mathcal{E} \rightarrow \mathcal{K}$ onto $\mathcal{K}$ from a direct sum $\mathcal{E}$ of locally free sheaves of finite rank on $X$. Hence for any quasicoherent graded $\mathcal{B}$-module $\mathcal{M}$ there is a surjective morphism onto $\mathcal{M}$ from a flat quasicoherent graded $\mathcal{B}$-module $\mathcal{P}=\bigoplus_{n} \mathcal{B} \otimes_{\mathcal{O}_{X}} \mathcal{E}_{n}[n]$, and for any quasicoherent CDG-module $\mathcal{M}$ over $\mathcal{B}$ there is a surjective closed morphism onto $\mathcal{M}$ from the CDG-module $G^{+}(\mathcal{P}) \in \mathcal{B}$-qcoh fl $_{\text {. }}$. (In fact, parts (a) and (b) of this theorem can be proven without the assumption of enough vector bundles on $X$ since there are always enough flat sheaves; see Remark 2.6 and Lemma A.1.)

Now the construction from [loc. cit., proof of Theorem 3.6] provides for any

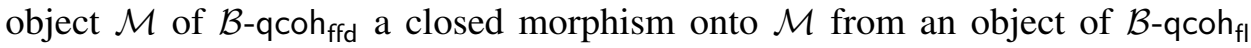
with the cone absolutely acyclic with respect to $\mathcal{B}$-qcoh ${ }_{\text {ffd }}$. To obtain this morphism, one picks a finite left resolution of $\mathcal{M}$ consisting of objects from $\mathcal{B}$-qcoh ${ }_{\mathrm{fl}}$ with closed morphisms between them, and takes the total CDG-module of this resolution. By [loc. cit., Lemma 1.6], it follows that the triangulated category $\mathrm{D}^{\mathrm{co}}\left(\mathcal{B}\right.$-qcoh ffd $\left._{\text {ff }}\right)$ is equivalent to the quotient category of $H^{0}\left(\mathcal{B}\right.$-qcoh $\left.{ }_{\mathrm{fl}}\right)$ by its intersection in $H^{0}\left(\mathcal{B}\right.$-qcoh $\left.{ }_{\text {ffd }}\right)$ with the thick subcategory of CDG-modules coacyclic

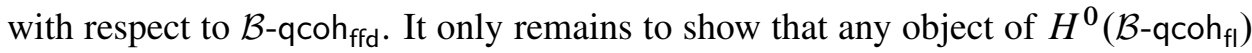
that is coacyclic with respect to $\mathcal{B}$-qcoh ffd $_{\text {is }}$ coacyclic with respect to $\mathcal{B}$-qcoh fl $_{\text {. }}$.

Let us call a quasicoherent CDG-module $\mathcal{M}$ over $\mathcal{B} d$-flat if its underlying quasicoherent graded $\mathcal{B}$-module $\mathcal{M}$ has flat dimension not exceeding $d$. A $d$-flat quasicoherent CDG-module is said to be $d$-coacyclic if it is homotopy equivalent to a CDG-module obtained from the total CDG-modules of exact triples of $d$-flat 
CDG-modules using the operations of cone and infinite direct sum. Our goal is to show that any 0 -flat $d$-coacyclic CDG-module is 0 -coacyclic. For this purpose, we will prove that any $(d-1)$-flat $d$-coacyclic CDG-module is $(d-1)$-coacyclic; the desired assertion will then follow by induction.

It suffices to construct for any $d$-coacyclic CDG-module $\mathcal{M}$ a $(d-1)$-coacyclic CDG-module $\mathcal{L}$ with a $(d-1)$-coacyclic $\mathrm{CDG}$-submodule $\mathcal{K}$ such that the quotient CDG-module $\mathcal{L} / \mathcal{K}$ is isomorphic to $\mathcal{M}$. Then if $\mathcal{M}$ is $(d-1)$-flat, it would follow that both the cone of the morphism $\mathcal{K} \rightarrow \mathcal{L}$ and the total CDG-module of the exact triple $\mathcal{K} \rightarrow \mathcal{L} \rightarrow \mathcal{M}$ are $(d-1)$-coacyclic, so $\mathcal{M}$ also is. The construction is based on four lemmas similar to those in [Polishchuk and Positselski 2012, Section 3.2].

Lemma A. Let $\mathcal{M}$ be the total CDG-module of an exact triple of $d$-flat quasicoherent $C D G$-modules $\mathcal{M}^{\prime} \rightarrow \mathcal{M}^{\prime \prime} \rightarrow \mathcal{M}^{\prime \prime \prime}$ over $\mathcal{B}$. Then there exists a surjective closed morphism onto $\mathcal{M}$ from a contractible 0 -flat $C D G$-module $\mathcal{P}$ with a $(d-1)$ coacyclic kernel $\mathcal{K}$.

Proof. Choose 0-flat quasicoherent CDG-modules $\mathcal{P}^{\prime}$ and $\mathcal{P}^{\prime \prime \prime}$ such that there exist surjective closed morphisms $\mathcal{P}^{\prime} \rightarrow \mathcal{M}^{\prime}$ and $\mathcal{P}^{\prime \prime \prime} \rightarrow \mathcal{M}^{\prime \prime}$. Then there exists a surjective morphism from the exact triple of CDG-modules $\mathcal{P}^{\prime} \rightarrow \mathcal{P}^{\prime} \oplus \mathcal{P}^{\prime \prime \prime} \rightarrow \mathcal{P}^{\prime \prime \prime}$ onto the exact triple $\mathcal{M}^{\prime} \rightarrow \mathcal{M}^{\prime \prime} \rightarrow \mathcal{M}^{\prime \prime \prime}$. The rest of the proof is similar to that in [Polishchuk and Positselski 2012].

Lemma B. (a) Let $\mathcal{K}^{\prime} \subset \mathcal{L}^{\prime}$ and $\mathcal{K}^{\prime \prime} \subset \mathcal{L}^{\prime \prime}$ be $(d-1)$-coacyclic $C D G$-submodules in $(d-1)$-coacyclic $C D G$-modules, and let $\mathcal{L}^{\prime} / \mathcal{K}^{\prime} \rightarrow \mathcal{L}^{\prime \prime} / \mathcal{K}^{\prime \prime}$ be a closed morphism of $C D G$-modules. Then there exists a $(d-1)$-coacyclic $C D G$-module $\mathcal{L}$ with a $(d-1)$-coacyclic $C D G$-submodule $\mathcal{K}$ such that

$$
\mathcal{L} / \mathcal{K} \simeq \operatorname{cone}\left(\mathcal{L}^{\prime} / \mathcal{K}^{\prime} \rightarrow \mathcal{L}^{\prime \prime} / \mathcal{K}^{\prime \prime}\right)
$$

(b) In the situation of (a), assume that the morphism $\mathcal{L}^{\prime} / \mathcal{K}^{\prime} \rightarrow \mathcal{L}^{\prime \prime} / \mathcal{K}^{\prime \prime}$ is injective with a d-flat cokernel $\mathcal{M}_{0}$. Then there exists a $(d-1)$-coacyclic $C D G$-module $\mathcal{L}_{0}$ with a $(d-1)$-coacyclic CDG-submodule $\mathcal{K}_{0}$ such that $\mathcal{L}_{0} / \mathcal{K}_{0} \simeq \mathcal{M}_{0}$.

Proof. The proof is similar to that in [Polishchuk and Positselski 2012].

Lemma C. For any contractible $d$-flat $C D G$-module $\mathcal{M}$ there exists a surjective closed morphism onto $\mathcal{M}$ from a contractible 0-flat CDG-module $\mathcal{L}$ with a $(d-1)$-coacyclic kernel $\mathcal{K}$.

Proof. Let $p: \mathcal{P} \rightarrow \mathcal{M}$ be a surjective morphism onto the quasicoherent graded $\mathcal{B}$-module $\mathcal{M}$ from a flat quasicoherent graded $\mathcal{B}$-module $\mathcal{P}$, and $\tilde{p}: G^{+}(\mathcal{P}) \rightarrow \mathcal{M}$ be the induced surjective closed morphism of quasicoherent CDG-modules. Let $t: \mathcal{M} \rightarrow \mathcal{M}$ be a contracting homotopy for $\mathcal{M}$ and $t_{\mathcal{P}}: G^{+}(\mathcal{P}) \rightarrow G^{+}(\mathcal{P})$ be the natural contracting homotopy for $G^{+}(\mathcal{P})$. Then $\tilde{u}=\tilde{p} t_{\mathcal{P}}-t \tilde{p}: G^{+}(\mathcal{P}) \rightarrow \mathcal{M}$ is a closed morphism of quasicoherent CDG-modules of degree -1 . Denote by $u$ 
the restriction of $\tilde{u}$ to $\mathcal{P} \subset G^{+}(\mathcal{P})$. There exists a surjective morphism from a flat quasicoherent graded $\mathcal{B}$-module $\mathcal{Q}$ onto the fibered product of the morphisms $p: \mathcal{P} \rightarrow \mathcal{M}$ and $u: \mathcal{P} \rightarrow \mathcal{M}$. Hence we obtain a surjective morphism of quasicoherent graded $\mathcal{B}$-modules $q: \mathcal{Q} \rightarrow \mathcal{P}$ and a morphism of quasicoherent graded $\mathcal{B}$-modules $v: \mathcal{Q} \rightarrow \mathcal{P}$ of degree -1 such that $u q=p v$.

The morphism $q$ induces a surjective closed morphism of quasicoherent CDGmodules $\tilde{q}: G^{+}(\mathcal{Q}) \rightarrow G^{+}(\mathcal{P})$. The morphism $\tilde{q}$ is homotopic to zero with the natural contracting homotopy $\tilde{q} t_{\mathcal{Q}}=t_{\mathcal{P}} \tilde{q}$. The morphism $v$ induces a closed morphism of CDG-modules $\tilde{v}: G^{+}(\mathcal{Q}) \rightarrow G^{+}(\mathcal{P})$ of degree -1 . The morphism $t_{\mathcal{P}} \tilde{q}-\tilde{v}$ is another contracting homotopy for $\tilde{q}$. The latter homotopy forms a commutative square with the morphisms $\tilde{p}, \tilde{p} \tilde{q}$, and the contracting homotopy $t$ for the CDG-module $\mathcal{M}$.

Let $\mathcal{N}$ be the kernel of the morphism $\tilde{p} \tilde{q}: G^{+}(\mathcal{Q}) \rightarrow \mathcal{M}$ and $\mathcal{K}$ be the kernel of the morphism $\tilde{p}: G^{+}(\mathcal{P}) \rightarrow \mathcal{M}$. Then the natural surjective closed morphism $r: \mathcal{N} \rightarrow \mathcal{K}$ is homotopic to zero; the restriction of the map $t_{\mathcal{P}} \tilde{q}-\tilde{v}$ provides the contracting homotopy that we need. In addition, the kernel $G^{+}(\operatorname{ker} q)$ of the morphism $r$ is contractible. So the cone of the morphism $r$ is isomorphic to $\mathcal{K} \oplus \mathcal{N}[1]$, and on the other hand, there is an exact triple $G^{+}(\operatorname{ker} q)[1] \rightarrow \operatorname{cone}(r) \rightarrow \operatorname{cone}\left(\mathrm{id}_{\mathcal{K}}\right)$. Since $\mathcal{K}$ is $(d-1)$-flat and $\operatorname{ker} q$ is flat, this proves that $\mathcal{K}$ is $(d-1)$-coacyclic. It remains to take $\mathcal{L}=G^{+}(\mathcal{P})$.

Lemma D. Let $\mathcal{M} \rightarrow \mathcal{M}^{\prime}$ be a homotopy equivalence of $d$-flat CDG-modules such that $\mathcal{M}^{\prime}$ is the quotient $C D G$-module of a $(d-1)$-coacyclic $C D G$-module by a $(d-1)$-coacyclic $C D G$-submodule. Then $\mathcal{M}$ is also such a quotient.

Proof. The proof is similar to that in [Polishchuk and Positselski 2012].

It is clear that the property of a CDG-module to be presentable as the cokernel of an injective closed morphism of $(d-1)$-coacyclic CDG-modules is stable under infinite direct sums. This finishes our construction and the proof of Theorem.

Remark 1.4. The assertion of part (c) of Theorem 1.4 can be equivalently rephrased with flat modules replaced by locally projective ones. Indeed, a finitely presented module over a ring is flat if and only if it is projective.

In the infinitely generated situation of parts (a) and (b), flatness of quasicoherent sheaves is different from their local projectivity (which is a stronger condition), but the assertions remain true after one replaces the former with the latter. The same applies to Proposition 1.5(a) below. Indeed, by Theorem A.2, for any quasicoherent graded algebra $\mathcal{B}$ over an affine scheme $U$, projectivity of a graded module over the graded ring $\mathcal{B}(U)$ is a local notion. Taking this fact into account, our proof goes through for locally projective quasicoherent graded modules in place of flat ones and the locally projective dimension (defined as the minimal length of a locally projective resolution) in place of the flat dimension. 
When $\mathcal{B}=\mathcal{O}_{X}$, local projectivity of quasicoherent modules is equivalent to local freeness [Bass 1963, Corollary 4.5]. Furthermore, in this case, assuming additionally that $X$ has finite Krull dimension, the classes of quasicoherent sheaves of finite flat dimension and of finite locally projective dimension coincide [Raynaud and Gruson 1971, Corollaire II.3.3.2].

1.5. Fully faithful embedding. The next proposition is stronger than Theorem 1.4 in some respects, and is proven by an entirely different technique.

Proposition 1.5. (a) For any quasicoherent $C D G$-algebra $\mathcal{B}$ over $X$, the functor $\mathrm{D}^{\mathrm{abs}}\left(\mathcal{B}\right.$-qcoh $\left.{ }_{\mathrm{fl}}\right) \rightarrow \mathrm{D}^{\mathrm{abs}}(\mathcal{B}$-qcoh) induced by the embedding of $D G$-categories $\mathcal{B}$-qcoh ${ }_{\mathrm{fl}} \rightarrow \mathcal{B}$-qcoh is fully faithful.

Furthermore, let $\mathcal{B}$ be a quasicoherent $C D G$-algebra over $X$ such that the underlying quasicoherent graded algebra $\mathcal{B}$ is Noetherian. Then

(b) the functor $\mathrm{D}^{\mathrm{abs}}\left(\mathcal{B}\right.$-coh $\left.\mathrm{fl}_{\mathrm{fl}}\right) \rightarrow \mathrm{D}^{\mathrm{abs}}(\mathcal{B}$-coh) induced by the embedding of $D G$-categories $\mathcal{B}$-coh ${ }_{\mathrm{fl}} \rightarrow \mathcal{B}$-coh is fully faithful;

(c) the functor $\mathrm{D}^{\mathrm{abs}}(\mathcal{B}$-coh $) \rightarrow \mathrm{D}^{\text {abs }}(\mathcal{B}$-qcoh) induced by the embedding of $D G$-categories $\mathcal{B}$-coh $\rightarrow \mathcal{B}$-qcoh is fully faithful;

(d) the functor $\mathrm{D}^{\mathrm{abs}}(\mathcal{B}$-coh $) \rightarrow \mathrm{D}^{\mathrm{co}}(\mathcal{B}$-qcoh $)$ induced by the embedding of $D G$-categories $\mathcal{B}$-coh $\rightarrow \mathcal{B}$-qcoh is fully faithful and its image forms a set of compact generators for $\mathrm{D}^{\mathrm{co}}(\mathcal{B}$-qcoh).

Proof. The proof of part (d) in the case when $X$ is affine can be found in [Positselski 2011b, Section 3.11] (the part concerning compact generation belongs to D. Arinkin). The proof in the general case is similar, and part (c) can be also proven in the way similar to [loc. cit., Theorem 3.11.1]. Part (b) in the affine case is easy and follows from the semiorthogonality property of CDG-modules with projective underlying graded modules and absolutely acyclic/contraacyclic CDG-modules [loc. cit., Theorem 3.5(b)] since finitely generated flat modules over a Noetherian ring are projective. A detailed proof of part (b) in the general case is given below; and the proof of part (a) (which does not automatically simplify in the affine case) is similar.

We will show that any morphism $\mathcal{E} \rightarrow \mathcal{L}$ from a CDG-module $\mathcal{E} \in H^{0}(\mathcal{B}$-coh fl $)$ to a CDG-module $\mathcal{L} \in H^{0}$ ( $\mathcal{B}$-coh) absolutely acyclic with respect to $\mathcal{B}$-coh can be annihilated by a morphism $\mathcal{P} \rightarrow \mathcal{E}$ from a CDG-module $\mathcal{P} \in H^{0}\left(\mathcal{B}\right.$ - coh $\left._{\mathrm{fl}}\right)$ with a cone of the morphism $\mathcal{P} \rightarrow \mathcal{E}$ being absolutely acyclic with respect to $\mathcal{B}$-coh fl $_{\mathrm{fl}}$. By the definition, the CDG-module $\mathcal{L}$ is a direct summand of a CDG-module homotopy equivalent to a CDG-module obtained from the totalizations of exact triples of CDG-modules in $\mathcal{B}$-coh using the operation of passage to the cone of a closed morphism repeatedly. It suffices to consider the case when $\mathcal{L}$ itself is obtained from totalizations of exact triples using cones. We proceed by induction in the number of operations of passage to the cone in such a construction of $\mathcal{L}$. 
So we assume that there is a distinguished triangle $\mathcal{K} \rightarrow \mathcal{L} \rightarrow \mathcal{M} \rightarrow \mathcal{K}[1]$ in $H^{0}(\mathcal{B}$-coh) such that $\mathcal{M}$ is the total CDG-module of an exact triple of CDG-modules in $\mathcal{B}$-coh, while the CDG-module $\mathcal{K}$ has the desired property with respect to morphisms into it from all CDG-modules $\mathcal{F} \in H^{0}\left(\mathcal{B}\right.$ - coh $\left._{\text {fl }}\right)$. If we knew that the object $\mathcal{M}$ also has the same property, it would follow that the composition $\mathcal{E} \rightarrow \mathcal{L} \rightarrow \mathcal{M}$ can be annihilated by a morphism $\mathcal{F} \rightarrow \mathcal{E}$ with $\mathcal{F} \in H^{0}\left(\mathcal{B}\right.$ - $\left.\operatorname{coh}_{\mathrm{fl}}\right)$ and a cone absolutely acyclic with respect to $\mathcal{B}$ - $\operatorname{coh}_{\mathrm{fl}}$. The composition $\mathcal{F} \rightarrow \mathcal{E} \rightarrow \mathcal{L}$ then factorizes through $\mathcal{K}$, and the morphism $\mathcal{F} \rightarrow \mathcal{K}$ can be annihilated by a morphism $\mathcal{P} \rightarrow \mathcal{F}$ with $\mathcal{P} \in H^{0}(\mathcal{B}$-coh fl $)$ and a cone absolutely acyclic with respect to $\mathcal{B}$ - $\operatorname{coh}_{\mathrm{fl}}$. The composition $\mathcal{P} \rightarrow \mathcal{F} \rightarrow \mathcal{E}$ provides the desired morphism $\mathcal{P} \rightarrow \mathcal{E}$.

Thus it remains to construct a morphism $\mathcal{F} \rightarrow \mathcal{E}$ with the required properties annihilating a morphism $\mathcal{E} \rightarrow \mathcal{M}$, where $\mathcal{M}$ is the total CDG-module of an exact triple of CDG-modules $\mathcal{U} \rightarrow \mathcal{V} \rightarrow \mathcal{W}$. For any graded module $\mathcal{N}$ over $\mathcal{B}$, morphisms of graded $\mathcal{B}$-modules $\mathcal{N} \rightarrow \mathcal{M}$ of degree $n$ are represented by triples $(f, g, h)$, where $f: \mathcal{N} \rightarrow \mathcal{U}$ is a morphism of degree $n+1, g: \mathcal{N} \rightarrow \mathcal{V}$ is a morphism of degree $n$, and $h: \mathcal{N} \rightarrow \mathcal{W}$ is a morphism of degree $n-1$. Denote the closed morphisms in the exact triple $\mathcal{U} \rightarrow \mathcal{V} \rightarrow \mathcal{W}$ by $j: \mathcal{U} \rightarrow \mathcal{V}$ and $k: \mathcal{V} \rightarrow \mathcal{W}$.

Lemma E. Let $\mathcal{N}$ be a $C D G$-module over $\mathcal{B}$ and $\mathcal{M}$ be the total CDG-module of an exact triple of $C D G$-modules $\mathcal{U} \rightarrow \mathcal{V} \rightarrow \mathcal{W}$ as above. Then

(a) the differential of a morphism of graded $\mathcal{B}$-modules $\mathcal{N} \rightarrow \mathcal{M}$ of degree $n$ represented by a triple $(f, g, h)$ is given by the rule

$$
d(f, g, h)=(-d f,-j f+d g, k g-d h) ;
$$

(b) when $(f, g, h)$ is a closed morphism of CDG-modules of degree $n$ and the morphism of graded $\mathcal{B}$-modules $h: \mathcal{N} \rightarrow \mathcal{W}$ can be lifted to a morphism of graded $\mathcal{B}$-modules $t: \mathcal{N} \rightarrow \mathcal{V}$ of degree $n-1$, the morphism $(f, g, h)$ is homotopic to zero.

Proof. We know that the complex of morphisms in the DG-category of CDG-modules $\operatorname{Hom}_{\mathcal{B}}(\mathcal{N}, \mathcal{M})$ is the total complex of the bicomplex of abelian groups

$$
\operatorname{Hom}_{\mathcal{B}}(\mathcal{N}, \mathcal{U}) \longrightarrow \operatorname{Hom}_{\mathcal{B}}(\mathcal{N}, \mathcal{V}) \longrightarrow \operatorname{Hom}_{\mathcal{B}}(\mathcal{N}, \mathcal{W})
$$

The formula in (a) is the formula for the differential of a total complex.

Furthermore, the sequence $0 \rightarrow \operatorname{Hom}_{\mathcal{B}}(\mathcal{N}, \mathcal{U}) \rightarrow \operatorname{Hom}_{\mathcal{B}}(\mathcal{N}, \mathcal{V}) \rightarrow \operatorname{Hom}_{\mathcal{B}}(\mathcal{N}, \mathcal{W})$ is exact. Let $\operatorname{Hom}_{\mathcal{B}}^{\prime}(\mathcal{N}, \mathcal{W})$ denote the cokernel of the morphism of complexes $\operatorname{Hom}_{\mathcal{B}}(\mathcal{N}, \mathcal{U}) \rightarrow \operatorname{Hom}_{\mathcal{B}}(\mathcal{N}, \mathcal{V})$; then $\operatorname{Hom}_{\mathcal{B}}^{\prime}(\mathcal{N}, \mathcal{W})$ is a subcomplex of $\operatorname{Hom}_{\mathcal{B}}(\mathcal{N}, \mathcal{W})$ and the total complex of the bicomplex

$$
\operatorname{Hom}_{\mathcal{B}}(\mathcal{N}, \mathcal{U}) \longrightarrow \operatorname{Hom}_{\mathcal{B}}(\mathcal{N}, \mathcal{V}) \longrightarrow \operatorname{Hom}_{\mathcal{B}}^{\prime}(\mathcal{N}, \mathcal{W})
$$


is an acyclic subcomplex of $\operatorname{Hom}_{\mathcal{B}}(\mathcal{N}, \mathcal{M})$. Hence any cocycle in $\operatorname{Hom}_{\mathcal{B}}(\mathcal{N}, \mathcal{M})$ that belongs to this subcomplex is a coboundary.

To present the same argument using our letter notation for morphisms, assume that $k t=h$. Then $k(d t-g)=d h-k g=0$, so there exists a morphism of graded $\mathcal{B}$-modules $s: \mathcal{N} \rightarrow \mathcal{U}$ of degree $n$ such that $d t-g=j s$. Then $j d s=-d g=-j f$; hence $d s=-f$ and $d(s, t, 0)=(f, g, h)$.

Recall the notation $G^{+}(\mathcal{Q})$ for the CDG-module freely generated by a graded $\mathcal{B}$-module $\mathcal{Q}$ (see the beginning of the proof of Theorem 1.4).

Lemma $\mathbf{F}$. Let $\mathcal{M}$ be the total $C D G$-module of an exact triple of $C D G$-modules $\mathcal{U} \rightarrow \mathcal{V} \rightarrow \mathcal{W}$ as above, and let $\mathcal{Q}$ be a graded $\mathcal{B}$-module. Assume that a morphism of graded $\mathcal{B}$-modules $p: \mathcal{Q} \rightarrow \mathcal{M}$ of degree $n$ with the components $(f, g, h)$ is given such that the component $h: \mathcal{Q} \rightarrow \mathcal{W}$ can be lifted to a morphism of graded $\mathcal{B}$-modules $t: \mathcal{Q} \rightarrow \mathcal{V}$ of degree $n-1$. Let $\tilde{p}: G^{+}(\mathcal{Q}) \rightarrow \mathcal{M}$ be the induced closed morphism of $C D G$-modules of degree $n$ and $(\tilde{f}, \tilde{g}, \tilde{h})$ be its three components. Then the morphism of graded $\mathcal{B}$-modules $\tilde{h}: G^{+}(\mathcal{Q}) \rightarrow \mathcal{W}$ can be lifted to a morphism of graded $\mathcal{B}$-modules $\tilde{t}: G^{+}(\mathcal{Q}) \rightarrow \mathcal{V}$ of degree $n-1$.

Proof. Notice that any closed morphism of CDG-modules $G^{+}(\mathcal{Q}) \rightarrow \mathcal{M}$ is homotopic to zero since the CDG-module $G^{+}(\mathcal{Q})$ is contractible. The conclusion of the lemma is stronger, and we will need its full strength. The argument consists of a computation in the letter notation for morphisms.

For any CDG-module $\mathcal{N}$ over $\mathcal{B}$, morphisms of graded $\mathcal{B}$-modules

$$
\tilde{r}: G^{+}(\mathcal{Q}) \longrightarrow \mathcal{N}
$$

of degree $n-1$ are uniquely determined by their restriction to $\mathcal{Q}$ and the restriction to $\mathcal{Q}$ of their differential $d \tilde{r}$, which can be arbitrary morphisms of graded $\mathcal{B}$-modules $\mathcal{Q} \rightarrow \mathcal{N}$ of degrees $n-1$ and $n$, respectively. Extend our morphism $t: \mathcal{Q} \rightarrow \mathcal{V}$ to a morphism of graded $\mathcal{B}$-modules $\tilde{t}: G^{+}(\mathcal{Q}) \rightarrow \mathcal{V}$ of degree $n-1$ such that $\left.(d \tilde{t})\right|_{\mathcal{Q}}=g$. Then $\left.k \tilde{t}\right|_{\mathcal{Q}}=k t=h=\left.\tilde{h}\right|_{\mathcal{Q}}$ and $\left.(d(k \tilde{t}))\right|_{\mathcal{Q}}=\left.k(d \tilde{t})\right|_{\mathcal{Q}}=k g=\left.k \tilde{g}\right|_{\mathcal{Q}}=\left.(d \tilde{h})\right|_{\mathcal{Q}}$ by Lemma $\mathrm{E}(\mathrm{a})$, and hence $k \tilde{t}=\tilde{h}$.

Now represent a closed morphism $\mathcal{E} \rightarrow \mathcal{M}$ by a triple $(f, g, h)$ of morphisms of degrees 1,0 , and -1 , respectively. Let $\mathcal{Q}$ be a flat coherent graded $\mathcal{B}$-module mapping surjectively onto the fibered product of the morphisms $k: \mathcal{V} \rightarrow \mathcal{W}$ and $h: \mathcal{E} \rightarrow \mathcal{W}$ (see the beginning of the proof of Theorem 1.4 again). Then there is a surjective morphism of graded $\mathcal{B}$-modules $q: \mathcal{Q} \rightarrow \mathcal{E}$ and its composition with the morphism $h: \mathcal{E} \rightarrow \mathcal{W}$ can be lifted to a morphism of graded $\mathcal{B}$-modules $t: \mathcal{Q} \rightarrow \mathcal{V}$ of degree -1 . Consider the induced morphism of CDG-modules $\tilde{q}: G^{+}(\mathcal{Q}) \rightarrow \mathcal{E}$. By Lemma F, the composition $h \tilde{q}: G^{+}(\mathcal{Q}) \rightarrow \mathcal{W}$ can be lifted to a morphism of graded $\mathcal{B}$-modules $\tilde{t}: G^{+}(\mathcal{Q}) \rightarrow \mathcal{V}$ of degree -1 . 
Let $\mathcal{R}$ denote the kernel of the closed morphism $\tilde{q}$. Then the cone $\mathcal{F}$ of the embedding $\mathcal{R} \rightarrow G^{+}(\mathcal{Q})$ maps naturally onto $\mathcal{E}$ with the cone absolutely acyclic

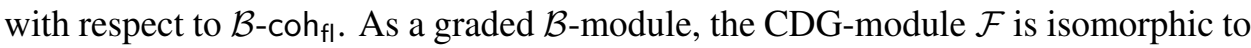
$G^{+}(\mathcal{Q}) \oplus \mathcal{R}[1]$; the composition $\mathcal{F} \rightarrow \mathcal{E} \rightarrow \mathcal{M}$ factorizes through the direct summand $G^{+}(\mathcal{Q})$, where it is defined by the triple $(f \tilde{q}, g \tilde{q}, h \tilde{q})$. Since the morphism $h \tilde{q}$ can be lifted to $\mathcal{V}$, so can the corresponding component $\mathcal{F} \rightarrow \mathcal{W}$ of the morphism $\mathcal{F} \rightarrow \mathcal{M}$. Thus the latter morphism is homotopic to zero by Lemma $\mathrm{E}(\mathrm{b})$.

In some cases, the use of Lemma $\mathrm{F}$ in the above proof of part (b) can be avoided. Assume that $X$ is a projective scheme over a Noetherian ring and the category of coherent graded $\mathcal{B}$-modules is equivalent to the category of coherent modules over some coherent (graded) $\mathcal{O}_{X}$-algebra $\mathcal{A}$. In this situation, one takes $\mathcal{Q}$ to be the graded $\mathcal{B}$-module corresponding to the (graded) $\mathcal{A}$-module induced from a large enough finite direct sum of (shifts of) copies of a sufficiently negative invertible $\mathcal{O}_{X}$-module; then there is a surjective morphism of graded $\mathcal{B}$-modules $\mathcal{Q} \rightarrow \mathcal{E}$ and any morphism of graded $\mathcal{B}$-modules $G^{+}(\mathcal{Q}) \rightarrow \mathcal{W}$ lifts to $\mathcal{V}$.

Remark 1.5. We do not know how to extend the proof of Proposition 1.5 (a) and (b) to the coderived categories of quasicoherent CDG-modules. Instead, this argument appears to be well-suited for use with the contraderived categories (see [Positselski 2011b, Section 3.3] for the definition). In particular, it allows to show that the contraderived category of left CDG-modules over a CDG-ring $B$ with a right coherent underlying graded ring is equivalent to the contraderived category of CDG-modules whose underlying graded $B$-modules are flat (cf. [loc. cit., paragraph after the proof of Theorem 3.8]).

This is the main reason why we sometimes find it easier to deal with the absolute derived rather than the coderived categories of infinitely generated CDG-modules (cf. Remark 2.8). On the other hand, for the coderived category of quasicoherent CDG-modules we have the compact generation result (part (d) of Proposition 1.5), the results and arguments of Sections 1.7, 1.10, 2.5, 2.9, etc. The conditions under which these two versions of the construction of the derived category of the second kind for a given class of CDG-modules lead to the same triangulated category are discussed below in Section 1.6.

1.6. Finite homological dimension theorem. Let $\mathcal{B}-\mathrm{qcoh}_{\mathrm{Ip}}$ denote the DG-category of quasicoherent $\mathrm{CDG}$-modules over $\mathcal{B}$ whose underlying graded $\mathcal{B}$-modules are locally projective (see Remark 1.4 and Theorem A.2). Denote by $\mathrm{D}^{\mathrm{co}}\left(\mathcal{B}\right.$-qcoh $\left.\mathrm{c}_{1 \mathrm{p}}\right)$ and $D^{\text {abs }}\left(\mathcal{B}\right.$-qcoh $\left.{ }_{1 \mathrm{p}}\right)$ the corresponding coderived and absolute derived categories.

Theorem 1.6. The triangulated categories $\mathrm{D}^{\mathrm{co}}\left(\mathcal{B}-\mathrm{q} \operatorname{coh}_{\mathrm{Ip}}\right)$ and $\mathrm{D}^{\mathrm{abs}}\left(\mathcal{B}\right.$-qcoh $\left.\mathrm{h}_{\mathrm{Ip}}\right)$ coincide; i.e., every $C D G$-module over $\mathcal{B}$ that is coacyclic with respect to $\mathcal{B}$-qcoh $\mathrm{h}_{\mathrm{Ip}}$ is also absolutely acyclic with respect to $\mathcal{B}$-qcoh ${ }_{1 \mathrm{p}}$. 
Proof. The reason for this assertion to be true is that the exact category of locally projective graded $\mathcal{B}$-modules has finite homological dimension [Orlov 2004, Lemma 1.12] and exact functors of infinite direct sums. If this exact category also had enough injectives, the simple argument from [Positselski 2011b, Theorem 3.6(a) and Remark 3.6] would suffice to establish the desired $D^{c o}=D^{\text {abs }}$ isomorphism for it (see also [Positselski 2010, Remark 2.1]). The lengthy argument below is designed to provide a way around the injective objects issue in this kind of proof.

Our aim is to show that for any closed morphism $\mathcal{P} \rightarrow \mathcal{L}$ from a CDG-module $\mathcal{P} \in \mathcal{B}$-qcoh Ip to a CDG-module $\mathcal{L}$ absolutely acyclic with respect to $\mathcal{B}$-qcoh ${ }_{\text {Ip }}$, there exists an exact sequence $0 \rightarrow \mathcal{Q}_{d} \rightarrow \mathcal{Q}_{d-1} \rightarrow \cdots \rightarrow \mathcal{Q}_{0} \rightarrow \mathcal{P} \rightarrow 0$ of CDG-modules

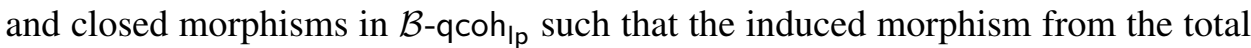
CDG-module of $\mathcal{Q}_{d} \rightarrow \cdots \rightarrow \mathcal{Q}_{0}$ to $\mathcal{L}$ is homotopic to zero. Here $d$ is a fixed integer equal to the homological dimension of the exact category of locally projective graded $\mathcal{B}$-modules, which does not exceed the number of open subsets in an affine covering of $X$ minus one.

Taking $\mathcal{P}=\mathcal{L}$ and the morphism $\mathcal{P} \rightarrow \mathcal{L}$ to be the identity, we will then conclude that $\mathcal{P}$ is isomorphic to a direct summand of the total CDG-module of $\mathcal{Q}_{d} \rightarrow \cdots \rightarrow$ $\mathcal{Q}_{0} \rightarrow \mathcal{P}$ in $H^{0}\left(\mathcal{B}\right.$-qcoh $\left.{ }_{1 \mathrm{p}}\right)$. Hence an object of $H^{0}\left(\mathcal{B}\right.$-qcoh $\left.{ }_{1 \mathrm{p}}\right)$ is absolutely acyclic with respect to $\mathcal{B}-q \operatorname{coh}_{1 \mathrm{p}}$ if and only if it is isomorphic to a direct summand of the total CDG-module of a $(d+2)$-term exact sequence of CDG-modules from $\mathcal{B}$-qcoh ${ }_{\text {Ip }}$ with closed morphisms between them. It will immediately follow that the class of CDG-modules absolutely acyclic with respect to $\mathcal{B}$-qcoh Ip $_{\text {Ip }}$ is closed under infinite direct sums, so it coincides with the class of coacyclic CDG-modules.

We can suppose that there exists a sequence of distinguished triangles

$$
\mathcal{K}_{i-1} \longrightarrow \mathcal{K}_{i} \longrightarrow \mathcal{M}_{i} \longrightarrow \mathcal{K}_{i-1}[1]
$$

in $H^{0}\left(\mathcal{B}\right.$-qcoh $\left.{ }_{\text {Ip }}\right)$ such that $\mathcal{K}_{0}=0, \mathcal{K}_{n}=\mathcal{L}$, and $\mathcal{M}_{i}$ is the total CDG-module of an exact triple $\mathcal{U}_{i} \rightarrow \mathcal{V}_{i} \rightarrow \mathcal{W}_{i}$ of CDG-modules from $\mathcal{B}$-qcoh lp for all $1 \leq i \leq n$. We will start with constructing an exact sequence $0 \rightarrow \mathcal{Q}_{n}^{\prime} \rightarrow \cdots \rightarrow \mathcal{Q}_{0}^{\prime} \rightarrow \mathcal{P} \rightarrow 0$ with the above properties, but of the length $n$ rather than $d$. Then we will use the finite homological dimension property of locally projective graded $\mathcal{B}$-modules in order to obtain the desired resolution $\mathcal{Q}$. of a fixed length $d$ from a resolution $\mathcal{Q}_{\bullet}^{\prime}$.

Lemma G. Let $\mathcal{M}$ be the total $C D G$-module of an exact triple $\mathcal{U} \rightarrow \mathcal{V} \rightarrow \mathcal{W}$ of $C D G$-modules from $\mathcal{B}$-qcoh ${ }_{1 \mathrm{p}}$ and $\mathcal{K} \rightarrow \mathcal{L} \rightarrow \mathcal{M} \rightarrow \mathcal{K}[1]$ be a distinguished triangle in $H^{0}(\mathcal{B}$-qcoh $1 \mathrm{p})$. Then for any $C D G$-module $\mathcal{P} \in \mathcal{B}$-qcoh ${ }_{\mathrm{lp}}$ and a morphism $\mathcal{P} \rightarrow \mathcal{L}$ in $H^{0}\left(\mathcal{B}\right.$-qcoh $\left.{ }_{1 \mathrm{p}}\right)$ there exists an exact triple $\mathcal{R} \rightarrow \mathcal{Q} \rightarrow \mathcal{P}$ of CDG-modules from $\mathcal{B}$-qcoh ${ }_{1 \mathrm{p}}$ and a morphism $\mathcal{R}[1] \rightarrow \mathcal{K}$ in $H^{0}\left(\mathcal{B}\right.$-qcoh $\left.{ }_{1 \mathrm{p}}\right)$ such that the composition $\mathcal{F} \rightarrow \mathcal{P} \rightarrow \mathcal{L}$, where $\mathcal{F}$ is the cone of the closed morphism $\mathcal{R} \rightarrow \mathcal{Q}$, is equal to the composition $\mathcal{F} \rightarrow \mathcal{R}[1] \rightarrow \mathcal{K} \rightarrow \mathcal{L}$ in $H^{0}\left(\mathcal{B}\right.$-qcoh $\left.{ }_{\text {Ip }}\right)$. 
Proof. The argument is based on Lemmas E and F from Section 1.5. We can assume that $\mathcal{L}$ is the cone of a closed morphism $\mathcal{M}[-1] \rightarrow \mathcal{K}$ and fix a closed morphism $\mathcal{P} \rightarrow \mathcal{L}$ representing the given morphism in the homotopy category. Arguing as in the proof of Proposition 1.5, we can construct a surjective closed morphism $\mathcal{Q}^{\prime} \rightarrow \mathcal{P}$ onto $\mathcal{P}$ from a CDG-module $\mathcal{Q}^{\prime} \in \mathcal{B}$-qcoh lp $_{\mathrm{p}}$ such that the composition $\mathcal{Q}^{\prime} \rightarrow \mathcal{P} \rightarrow \mathcal{L} \rightarrow \mathcal{M} \rightarrow \mathcal{W}[-1]$ lifts to a morphism of graded $\mathcal{B}$-modules $\mathcal{Q}^{\prime} \rightarrow \mathcal{V}[-1]$. Here it suffices to apply the functor $G^{+}$to the fibered product of the morphisms of graded $\mathcal{B}$-modules $\mathcal{P} \rightarrow \mathcal{W}[-1]$ and $\mathcal{V}[-1] \rightarrow \mathcal{W}[-1]$ and use Lemma $\mathrm{F}$.

Then the morphism $\mathcal{Q}^{\prime} \rightarrow \mathcal{M}$ is homotopic to zero with a natural contracting homotopy (provided by the proof of Lemma E), so the morphism $\mathcal{Q}^{\prime} \rightarrow \mathcal{L}$ factorizes, up to a homotopy, as the composition of a naturally defined closed morphism $\mathcal{Q}^{\prime} \rightarrow \mathcal{K}$ and the closed morphism $\mathcal{K} \rightarrow \mathcal{L}$. Set $\mathcal{Q}$ to be the cocone of the closed morphism $\mathcal{Q}^{\prime} \rightarrow \mathcal{K}$; then we have a surjective closed morphism $\mathcal{Q} \rightarrow \mathcal{Q}^{\prime}$ such that the composition $\mathcal{Q} \rightarrow \mathcal{Q}^{\prime} \rightarrow \mathcal{K}$ is homotopic to zero.

Let $\mathcal{R}$ be kernel of the morphism $\mathcal{Q} \rightarrow \mathcal{P}$ and $\mathcal{F}$ be the cone of the morphism $\mathcal{R} \rightarrow \mathcal{Q}$; then there is a natural closed morphism $\mathcal{F} \rightarrow \mathcal{P}$. Using Lemma $\mathrm{E}$ and arguing as in the end of the proof of Proposition 1.5 again, we can conclude that the composition $\mathcal{F} \rightarrow \mathcal{P} \rightarrow \mathcal{L} \rightarrow \mathcal{M}$ is homotopic to zero. Indeed, the composition $\mathcal{F} \rightarrow \mathcal{M} \rightarrow \mathcal{W}[-1]$ lifts to a graded $\mathcal{B}$-module morphism $\mathcal{F} \rightarrow \mathcal{V}[-1]$ since $\mathcal{F} \simeq$ $\mathcal{Q} \oplus \mathcal{R}[-1]$ as a graded $\mathcal{B}$-module, the morphism $\mathcal{F} \rightarrow \mathcal{M}$ factorizes through the projection of $\mathcal{F}$ onto $\mathcal{Q}$, and the morphism $\mathcal{Q} \rightarrow \mathcal{Q}^{\prime} \rightarrow \mathcal{W}[-1]$ lifts to a graded $\mathcal{B}$-module morphism $\mathcal{Q} \rightarrow \mathcal{Q}^{\prime} \rightarrow \mathcal{V}[-1]$ by our construction.

Notice that the contracting homotopy that we have obtained for the closed morphism $\mathcal{F} \rightarrow \mathcal{M}$ forms a commutative diagram with the closed morphisms $\mathcal{Q} \rightarrow \mathcal{F}, \mathcal{Q} \rightarrow \mathcal{Q}^{\prime}$, and the contracting homotopy that we have previously had for the closed morphism $\mathcal{Q}^{\prime} \rightarrow \mathcal{M}$ (since so do the liftings $\mathcal{F} \rightarrow \mathcal{V}[-1]$ and $\mathcal{Q}^{\prime} \rightarrow \mathcal{V}[-1]$ ). This allows to factorize, up to a homotopy, the closed morphism $\mathcal{F} \rightarrow \mathcal{L}$ as the composition of a closed morphism $\mathcal{F} \rightarrow \mathcal{K}$ and the closed morphism $\mathcal{K} \rightarrow \mathcal{L}$ in such a way that the morphism $\mathcal{F} \rightarrow \mathcal{K}$ forms a commutative diagram with the closed morphisms $\mathcal{Q} \rightarrow \mathcal{F}, \mathcal{Q} \rightarrow \mathcal{Q}^{\prime}$, and the closed morphism $\mathcal{Q}^{\prime} \rightarrow \mathcal{K}$ that we have previously constructed. The composition $\mathcal{Q} \rightarrow \mathcal{F} \rightarrow \mathcal{K}$, being equal to the composition $\mathcal{Q} \rightarrow \mathcal{Q}^{\prime} \rightarrow \mathcal{K}$, is homotopic to zero; hence the morphism $\mathcal{F} \rightarrow \mathcal{K}$ factorizes through the closed morphism $\mathcal{F} \rightarrow \mathcal{R}[1]$ in $H^{0}\left(\mathcal{B}\right.$-qcoh $\left.{ }_{\text {lp }}\right)$.

Applying Lemma $\mathrm{G}$ to the morphism $\mathcal{P} \rightarrow \mathcal{L}$ and the distinguished triangle $\mathcal{K}_{n-1} \rightarrow \mathcal{L} \rightarrow \mathcal{M}_{n} \rightarrow \mathcal{K}_{n-1}$, we obtain an exact triple $\mathcal{R}_{0}^{\prime} \rightarrow \mathcal{Q}_{0}^{\prime} \rightarrow \mathcal{P}$ and a morphism $\mathcal{R}_{0}^{\prime}[1] \rightarrow \mathcal{K}_{n-1}$ in $H^{0}\left(\mathcal{B}\right.$-qcoh $\left.{ }_{\text {lp }}\right)$. Applying the same lemma again to the morphism $\mathcal{R}_{0}^{\prime}[1] \rightarrow \mathcal{K}_{n-1}$ and the distinguished triangle $\mathcal{K}_{n-2} \rightarrow \mathcal{K}_{n-1} \rightarrow \mathcal{M}_{n-1} \rightarrow \mathcal{K}_{n-2}[1]$, we construct an exact triple $\mathcal{R}_{1}^{\prime} \rightarrow \mathcal{Q}_{1}^{\prime} \rightarrow \mathcal{R}_{0}^{\prime}$ and a morphism $\mathcal{R}_{1}^{\prime}[2] \rightarrow \mathcal{K}_{n-2}$, etc. Finally we obtain an exact triple $\mathcal{R}_{n-1}^{\prime} \rightarrow \mathcal{Q}_{n-1}^{\prime} \rightarrow \mathcal{R}_{n-2}^{\prime}$ and a morphism $\mathcal{R}_{n-1}^{\prime}[n] \rightarrow \mathcal{K}_{0}=0$. 
Let us check that the natural morphism from the total CDG-module of the complex $0 \rightarrow \mathcal{R}_{n-1}^{\prime} \rightarrow \mathcal{Q}_{n-1}^{\prime} \rightarrow \cdots \rightarrow \mathcal{Q}_{0}^{\prime}$ to the CDG-module $\mathcal{L}$ is homotopic to zero. Denote this morphism by $f_{n}$. It factorizes naturally through the cone $\mathcal{F}_{0}$ of the closed morphism $\mathcal{R}_{0}^{\prime} \rightarrow \mathcal{Q}_{0}^{\prime}$, and the morphism $\mathcal{F}_{0} \rightarrow \mathcal{L}$ is homotopic to the composition $\mathcal{F}_{0} \rightarrow \mathcal{R}_{0}^{\prime}[1] \rightarrow \mathcal{K}_{n-1} \rightarrow \mathcal{L}$. Hence, up to the homotopy, the morphism $f_{n}$ factorizes through the morphism $f_{n-1}$ from the total CDG-module of the complex $0 \rightarrow \mathcal{R}_{n-1}^{\prime} \rightarrow \mathcal{Q}_{n-1}^{\prime} \rightarrow \cdots \rightarrow \mathcal{Q}_{1}^{\prime}$ to $\mathcal{K}_{n-1}$ induced by the morphism $\mathcal{R}_{0}^{\prime}[1] \rightarrow \mathcal{K}_{n-1}$. Continuing to argue in this way, we conclude that the morphism $f$ factorizes, up to a homotopy, through the morphism $f_{0}: \mathcal{R}_{n-1}^{\prime}[n] \rightarrow \mathcal{K}_{0}=0$.

It remains to "cut" our exact sequence of an unknown length $n$ to a fixed size $d$. For this purpose, we will assume that $n>d$ and construct from our exact sequence of length $n$ another exact sequence with the same properties, but of the length $n-1$. This part of the argument is based on the following lemma.

Lemma $\mathbf{H}$. For any $C D G$-module $\mathcal{M} \in \mathcal{B}$-qcoh ${ }_{1 \mathrm{p}}$, locally projective graded $\mathcal{B}$-module $\mathcal{E}$, and a homogeneous surjective morphism of locally projective graded $\mathcal{B}$-modules $\mathcal{E} \rightarrow \mathcal{M}$, there exist a $C D G$-module $\mathcal{Q} \in \mathcal{B}$-qcoh ${ }_{1 \mathrm{p}}$, a surjective closed morphism of $C D G$-modules $\mathcal{Q} \rightarrow \mathcal{M}$, and a homogeneous surjective morphism of locally projective graded $\mathcal{B}$-modules $\mathcal{Q} \rightarrow \mathcal{E}$ such that the triangle $\mathcal{Q} \rightarrow \mathcal{E} \rightarrow \mathcal{M}$ commutes.

Proof. For any open subscheme $U \subset X$, one can simply define $\mathcal{Q}^{i}(U)$ as the abelian group of all pairs $\left(e^{\prime} \in \mathcal{E}^{i+1}(U), e \in \mathcal{E}^{i}(U)\right)$ such that $d f(e)=f\left(e^{\prime}\right)$, where $f$ denotes the morphism of graded $\mathcal{B}$-modules $\mathcal{E} \rightarrow \mathcal{M}$ and $d$ is the differential in $\mathcal{M}$. The action of $\mathcal{B}$ in $\mathcal{Q}$ is defined by the formula $b\left(e^{\prime}, e\right)=\left((-1)^{|b|} b e^{\prime}+d(b) e, b e\right)$; the differential in $\mathcal{Q}$ is given by the obvious rule $d\left(e^{\prime}, e\right)=\left(h e, e^{\prime}\right)$. The morphism $\mathcal{Q} \rightarrow \mathcal{E}$ is defined as $\left(e^{\prime}, e\right) \longmapsto e$; the morphism $\mathcal{Q} \rightarrow \mathcal{M}$, given by $\left(e^{\prime}, e\right) \longmapsto f(e)$, obviously commutes with the differentials.

It remains to check that the graded $\mathcal{B}$-module $\mathcal{Q}$ is locally projective. This can be done by comparing the above construction with the constructions of the freely (co)generated CDG-modules $G^{+}(\mathcal{E})$ and $G^{-}(\mathcal{E})$ from [Positselski 2011b, proof of Theorem 3.6] (see the beginning of the proof of Theorem 1.4). One can simply define $G^{-}(\mathcal{E})$ as being isomorphic to $G^{+}(\mathcal{E})[1]$. Since $\mathcal{M}$ is a CDG-module, there is a natural closed morphism of CDG-modules $\mathcal{M} \rightarrow G^{-}(\mathcal{M})$. The CDG-module $\mathcal{Q}$ is the fibered product of the surjective closed morphism of CDG-modules $G^{-}(\mathcal{E}) \rightarrow$ $G^{-}(\mathcal{M})$ and the closed morphism $\mathcal{M} \rightarrow G^{-}(\mathcal{M})$; hence the graded $\mathcal{B}$-module $\mathcal{Q}$ is locally projective. The morphism $\mathcal{Q} \rightarrow \mathcal{E}$ is induced by the natural morphism of graded $\mathcal{B}$-modules $G^{-}(\mathcal{E}) \rightarrow \mathcal{E}$. It forms a commutative diagram with the morphism $\mathcal{E} \rightarrow \mathcal{M}$, since the composition $\mathcal{M} \rightarrow G^{-}(\mathcal{M}) \rightarrow \mathcal{M}$ is the identity morphism.

The exact sequence of CDG-modules

$$
0 \longrightarrow \mathcal{R}_{n-1}^{\prime} \longrightarrow \mathcal{Q}_{n-1}^{\prime} \longrightarrow \cdots \longrightarrow \mathcal{Q}_{0}^{\prime} \longrightarrow \mathcal{P} \longrightarrow 0
$$


represents a certain Yoneda Ext class of degree $n$ between the locally projective graded $\mathcal{B}$-modules $\mathcal{P}$ and $\mathcal{R}_{n-1}^{\prime}$. Since the homological dimension of the exact category of such $\mathcal{B}$-modules is equal to $d$ and we assume that $n>d$, this Ext class has to vanish. This means that there exists an exact sequence of locally projective graded $\mathcal{B}$-modules $0 \rightarrow \mathcal{R}_{n-1}^{\prime} \rightarrow \mathcal{E}_{n-1} \rightarrow \cdots \rightarrow \mathcal{E}_{0} \rightarrow \mathcal{P} \rightarrow 0$ mapping to our original exact sequence, with the maps on the rightmost and leftmost terms being the identity maps, such that the embedding of $\mathcal{B}$-modules $\mathcal{R}_{n-1}^{\prime} \rightarrow \mathcal{E}_{n-1}$ splits.

As explained in [Positselski 2011a, proof of Lemma 4.4], one can assume the morphisms $\mathcal{E}_{i} \rightarrow \mathcal{Q}_{i}^{\prime}$ to be surjective. Applying Lemma $\mathrm{H}$, we obtain a surjective closed morphism of CDG-modules $\mathcal{Q}_{0} \rightarrow \mathcal{Q}_{0}^{\prime}$ and a morphism of graded $\mathcal{B}$-modules $\mathcal{Q}_{0} \rightarrow \mathcal{E}_{0}$ forming a commutative triangle with the morphism $\mathcal{E}_{0} \rightarrow \mathcal{Q}_{0}^{\prime}$. Applying Lemma $\mathrm{H}$ to the surjective morphism of fibered products $\mathcal{Q}_{0} \times \mathcal{E}_{0} \mathcal{E}_{1} \rightarrow \mathcal{Q}_{0} \times{ }_{\mathcal{Q}_{0}^{\prime}} \mathcal{Q}_{1}^{\prime}$, we obtain a surjective closed morphism $\mathcal{Q}_{1} \rightarrow \mathcal{Q}_{1}^{\prime}$ and a closed morphism $\mathcal{Q}_{1} \rightarrow \mathcal{Q}_{0}$ forming a commutative square with the closed morphisms $\mathcal{Q}_{0} \rightarrow \mathcal{Q}_{0}^{\prime}$ and $\mathcal{Q}_{1}^{\prime} \rightarrow \mathcal{Q}_{0}^{\prime}$. Besides, the sequence $\mathcal{Q}_{1} \rightarrow \mathcal{Q}_{0} \rightarrow \mathcal{P}$ is exact at $\mathcal{Q}_{0}$. We also obtain a morphism of graded $\mathcal{B}$-modules $\mathcal{Q}_{1} \rightarrow \mathcal{E}_{1}$ forming a commutative triangle with the morphisms to $\mathcal{Q}_{1}^{\prime}$ and a commutative square with the morphisms to $\mathcal{E}_{0}$.

Proceeding in this way, we construct a sequence $\mathcal{Q}_{n-2} \rightarrow \cdots \rightarrow \mathcal{Q}_{0} \rightarrow \mathcal{P} \rightarrow 0$, which is exact at all the middle terms, maps onto the sequence $\mathcal{Q}_{n-2}^{\prime} \rightarrow \cdots \rightarrow$ $\mathcal{Q}_{0}^{\prime} \rightarrow \mathcal{P}$ by closed morphisms, and maps into the sequence $\mathcal{E}_{n-2} \rightarrow \cdots \rightarrow \mathcal{E}_{0} \rightarrow \mathcal{P}$ so that the triangle of the maps of sequences commutes. Finally, notice that $\mathcal{E}_{n-1} \simeq \mathcal{E}_{n-2} \times_{\mathcal{Q}_{n-2}^{\prime}} \mathcal{Q}_{n-1}^{\prime}$, and set $\mathcal{Q}_{n-1}=\mathcal{Q}_{n-2} \times_{\mathcal{Q}_{n-2}^{\prime}} \mathcal{Q}_{n-1}^{\prime}$. Then the exact sequence of CDG-modules $0 \rightarrow \mathcal{R}_{n-1}^{\prime} \rightarrow \mathcal{Q}_{n-1} \rightarrow \cdots \rightarrow \mathcal{Q}_{0} \rightarrow \mathcal{P} \rightarrow 0$ maps onto the exact sequence $0 \rightarrow \mathcal{R}_{n-1}^{\prime} \rightarrow \mathcal{Q}_{n-1}^{\prime} \rightarrow \cdots \rightarrow \mathcal{Q}_{0}^{\prime} \rightarrow \mathcal{P} \rightarrow 0$ by closed morphisms, and this map of exact sequences factorizes through the exact sequence of graded $\mathcal{B}$-modules $0 \rightarrow \mathcal{R}_{n-1}^{\prime} \rightarrow \mathcal{E}_{n-1} \rightarrow \cdots \rightarrow \mathcal{E}_{0} \rightarrow \mathcal{P} \rightarrow 0$. The composition of the morphism $\mathcal{Q}_{n-1} \rightarrow \mathcal{E}_{n-1}$ with the splitting $\mathcal{E}_{n-1} \rightarrow \mathcal{R}_{n-1}^{\prime}$ of the embedding $\mathcal{R}_{n-1}^{\prime} \rightarrow$ $\mathcal{E}_{n-1}$ provides a graded $\mathcal{B}$-module splitting $\mathcal{Q}_{n-1} \rightarrow \mathcal{R}_{n-1}^{\prime}$ of the embedding of CDG-modules $\mathcal{R}_{n-1}^{\prime} \rightarrow \mathcal{Q}_{n-1}$.

Denote by $\mathcal{R}_{n-2}$ the image of the morphism of CDG-modules $\mathcal{Q}_{n-1} \rightarrow \mathcal{Q}_{n-2}$. The morphism from the total CDG-module of the complex $\mathcal{R}_{n-1}^{\prime} \rightarrow \mathcal{Q}_{n-1}^{\prime} \rightarrow \cdots \rightarrow \mathcal{Q}_{0}^{\prime}$ to the CDG-module $\mathcal{L}$ is homotopic to zero; hence so is the morphism to $\mathcal{L}$ from the total CDG-module of the complex $\mathcal{R}_{n-1}^{\prime} \rightarrow \mathcal{Q}_{n-1} \rightarrow \cdots \rightarrow \mathcal{Q}_{0}$. The latter morphism factorizes naturally through the total CDG-module of the complex $\mathcal{R}_{n-2} \rightarrow \mathcal{Q}_{n-2} \rightarrow \cdots \rightarrow \mathcal{Q}_{0}$. The cone of this closed morphism between two total CDG-modules is homotopy equivalent to the total CDG-module of the exact triple $\mathcal{R}_{n-1}^{\prime} \rightarrow \mathcal{Q}_{n-1} \rightarrow \mathcal{R}_{n-2}$. Since this exact triple splits as an exact triple of graded $\mathcal{B}$-modules, its total CDG-module is contractible. Consequently, the morphism between the total CDG-modules of $\mathcal{R}_{n-1}^{\prime} \rightarrow \mathcal{Q}_{n-1} \rightarrow \cdots \rightarrow \mathcal{Q}_{0}$ and $\mathcal{R}_{n-2} \rightarrow \mathcal{Q}_{n-2} \rightarrow \cdots \rightarrow \mathcal{Q}_{0}$ is a homotopy equivalence. 
It follows that the natural morphism from the total CDG-module of the resolution $\mathcal{R}_{n-2} \rightarrow \mathcal{Q}_{n-2} \rightarrow \cdots \rightarrow \mathcal{Q}_{0}$ of the CDG-module $\mathcal{P}$ to the CDG-module $\mathcal{L}$ is homotopic to zero, and we are done.

So far we have only considered flat coherent CDG-modules over quasicoherent CDG-algebras $\mathcal{B}$ whose underlying quasicoherent graded algebras are Noetherian. But the latter restriction is not necessary, as flat and locally finitely presented (or, which is equivalent, locally projective and finitely generated) quasicoherent graded $\mathcal{B}$-modules always form an exact subcategory of flat (or locally projective) graded $\mathcal{B}$-modules. The notation $\mathcal{B}$-coh ${ }_{1 \mathrm{p}}$ (understood in the obvious sense as the DG-category of CDG-modules over $\mathcal{B}$ with coherent and locally projective underlying graded $\mathcal{B}$-modules) is synonymous with $\mathcal{B}$-coh ${ }_{\mathrm{fl}}$ (see Remark 1.4).

Corollary 1.6. The functor $\mathrm{D}^{\text {abs }}\left(\mathcal{B}\right.$-coh $\left.\mathrm{cop}_{\mathrm{p}}\right) \rightarrow \mathrm{D}^{\mathrm{co}}\left(\mathcal{B}\right.$-qcoh $\left.\mathrm{c}_{\mathrm{lp}}\right)$ induced by the embedding of DG-categories $\mathcal{B}$-coh $\mathrm{C}_{\mathrm{p}} \rightarrow \mathcal{B}$-qcoh ${ }_{\mathrm{lp}}$ is fully faithful.

Proof. When $\mathcal{B}$ is Noetherian, one can show that the functor $\mathrm{D}^{\mathrm{abs}}\left(\mathcal{B}-\operatorname{coh}_{\mathrm{lp}}\right) \rightarrow$ $\mathrm{D}^{\mathrm{abs}}\left(\mathcal{B}\right.$-qcoh $\left.{ }_{\mathrm{Ip}}\right)$ is fully faithful by comparing parts (a)-(c) of Proposition 1.5 (with the flatness condition replaced by the local projectivity). In the general case, one proves this assertion directly, using an argument similar to the proof of Proposition 1.5(a) and (b). Then it remains to use Theorem 1.6.

When every flat quasicoherent graded module over $\mathcal{B}$ has finite locally projective dimension (see Remark 1.4), one has

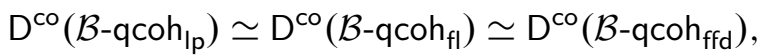

$$
\begin{aligned}
& \mathrm{D}^{\text {abs }}\left(\mathcal{B}-\text { qcoh }_{\mathrm{lp}}\right) \simeq \mathrm{D}^{\text {abs }}\left(\mathcal{B}-\mathrm{q} \operatorname{coh}_{\mathrm{fl}}\right) \simeq \mathrm{D}^{\text {abs }}\left(\mathcal{B}-\mathrm{qcoh}_{\mathrm{ffd}}\right)
\end{aligned}
$$

by appropriate versions of Theorem 1.4. Consequently, it follows from Theorem 1.6

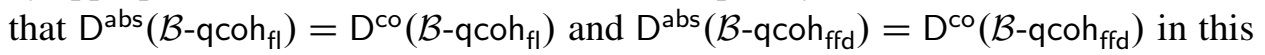
case. Thus the functor $\mathrm{D}^{\text {abs }}\left(\mathcal{B}-\operatorname{coh}_{\mathrm{fl}}\right) \rightarrow \mathrm{D}^{\mathrm{co}}\left(\mathcal{B}-\mathrm{qcoh}_{\mathrm{fl}}\right)$ is fully faithful; when $\mathcal{B}$ is Noetherian, so is the functor $D^{\text {abs }}\left(\mathcal{B}-\operatorname{coh}_{\mathrm{ffd}}\right) \rightarrow \mathrm{D}^{\mathrm{co}}\left(\mathcal{B}-\mathrm{qcoh}_{\mathrm{ffd}}\right)$.

1.7. Gorenstein case. Here we establish a sufficient condition for the functor $\mathrm{D}^{\mathrm{CO}}\left(\mathcal{B}\right.$-qcoh $\left.\mathrm{fl}_{\mathrm{fl}}\right) \rightarrow \mathrm{D}^{\mathrm{co}}(\mathcal{B}$-qcoh $)$ to be an equivalence of triangulated categories.

Let $\mathcal{B}$-qcoh ${ }_{\text {inj }}$ denote the full DG-subcategory in $\mathcal{B}$-qcoh consisting of the CDGmodules whose underlying quasicoherent graded $\mathcal{B}$-modules are injective. Furthermore, let $\mathcal{B}$-qcoh ${ }_{\text {fid }}$ be the full DG-subcategory in $\mathcal{B}$-qcoh consisting of the CDG-modules whose underlying quasicoherent graded $\mathcal{B}$-modules have finite injective dimension (i.e., admit a finite right resolution by injective quasicoherent graded $\mathcal{B}$-modules). Let $\mathrm{D}^{\mathrm{abs}}\left(\mathcal{B}\right.$-qcoh fid $\left._{\text {fid }}\right)$ and $\mathrm{D}^{\mathrm{co}}\left(\mathcal{B}\right.$-qcoh $\left.{ }_{\text {fid }}\right)$ denote the corresponding derived categories of the second kind. (The difficulty in the definition of the latter category, similar to the difficulty in the definition of $D^{\mathrm{co}}\left(\mathcal{B}\right.$-qcoh ffd $\left._{\text {f }}\right)$ discussed in Section 1.3, does not actually arise, as it is clear from part (a) of the next lemma.) 
Lemma 1.7. (a) For any quasicoherent $C D G$-algebra $\mathcal{B}$ over $X$, the natural functors $H^{0}\left(\mathcal{B}\right.$-qcoh $\left.{ }_{\text {inj }}\right) \rightarrow D^{\text {abs }}\left(\mathcal{B}\right.$-qcoh $\left.{ }_{\text {fid }}\right) \rightarrow D^{\mathrm{co}}\left(\mathcal{B}-\right.$ qcoh $\left._{\text {fid }}\right)$ are equivalences of triangulated categories.

(b) Let $\mathcal{B}$ be a quasicoherent $C D G$-algebra over $X$ whose underlying quasicoherent graded algebra $\mathcal{B}$ is Noetherian. Then the functor $H^{0}\left(\mathcal{B}-q c_{\mathrm{inj}}\right) \rightarrow$ $\mathrm{D}^{\mathrm{co}}\left(\mathcal{B}\right.$-qcoh) induced by the embedding $\mathcal{B}$-qcoh ${ }_{\mathrm{inj}} \rightarrow \mathcal{B}$-qcoh is an equivalence of triangulated categories.

Proof. Part (a) is provided by [Positselski 2011b, Theorem and Remark in Section 3.6]. Part (b) is a particular case of [loc. cit., Theorem and Remark in Section 3.7] since the class of injective quasicoherent graded $\mathcal{B}$-modules is closed under infinite direct sums in its assumptions. (Cf. [Lin and Pomerleano 2013, Proposition 2.4].)

Proposition 1.7. Let $\mathcal{B}$ be a quasicoherent $C D G$-algebra over $X$ such that the quasicoherent graded algebra $\mathcal{B}$ is Noetherian and the classes of quasicoherent graded $\mathcal{B}$-modules of finite flat dimension and of finite injective dimension coincide. Then the functors $\mathrm{D}^{\mathrm{abs}}(\mathcal{B}-\mathrm{qcoh} f \mathrm{fl}) \rightarrow \mathrm{D}^{\mathrm{co}}\left(\mathcal{B}-\mathrm{qcoh}_{\mathrm{fl}}\right) \rightarrow \mathrm{D}^{\mathrm{co}}(\mathcal{B}$-qcoh $)$ induced by the embedding $\mathcal{B}$-qcoh $\mathrm{fl}_{\mathrm{f}} \rightarrow \mathcal{B}$-qcoh are equivalences of triangulated categories.

Proof. Since $\mathcal{B}$-qcoh ${ }_{\mathrm{ffd}}=\mathcal{B}$-qcoh fid $_{\text {fid }}$, the isomorphism of categories $\mathrm{D}^{\text {abs }}\left(\mathcal{B}-\mathrm{qcoh}_{\mathrm{ffd}}\right)=$ $\mathrm{D}^{\mathrm{co}}\left(\mathcal{B}\right.$-qcoh $\left.{ }_{\mathrm{ffd}}\right)$ follows from part (a) of Lemma 1.7. Applying Theorem 1.4, we obtain the isomorphism of categories $D^{\text {abs }}\left(\mathcal{B}-q \operatorname{coh}_{\mathrm{fl}}\right) \rightarrow D^{\mathrm{co}}\left(\mathcal{B}-q\right.$ coh $\left._{\mathrm{fl}}\right)$. Similarly, it suffices to compare parts (a) and (b) of Lemma 1.7 in order to conclude that the functor $\mathrm{D}^{\mathrm{co}}\left(\mathcal{B}\right.$-qcoh $\left.\mathrm{qid}_{\mathrm{fid}}\right) \rightarrow \mathrm{D}^{\mathrm{co}}(\mathcal{B}$-qcoh $)$ is an equivalence of categories; hence so are the functors $\mathrm{D}^{\mathrm{co}}(\mathcal{B}-\mathrm{qcoh} f \mathrm{fl}) \rightarrow \mathrm{D}^{\mathrm{co}}\left(\mathcal{B}-\mathrm{qcoh}_{\mathrm{ffd}}\right) \rightarrow \mathrm{D}^{\mathrm{co}}(\mathcal{B}$-qcoh). (Cf. [Positselski 2011b, Section 3.9].)

1.8. Pull-backs and push-forwards. Let $f: Y \rightarrow X$ be a morphism of separated Noetherian schemes, $\mathcal{B}_{X}$ be a quasicoherent CDG-algebra over $X$, and $\mathcal{B}_{Y}$ be a quasicoherent CDG-algebra over $Y$. A morphism of quasicoherent CDG-algebras $\mathcal{B}_{X} \rightarrow \mathcal{B}_{Y}$ compatible with the morphism $Y \rightarrow X$ is the data of a CDG-ring morphism $\mathcal{B}_{X}(U) \rightarrow \mathcal{B}_{Y}(V)$ for each pair of affine open subschemes $U \subset X$ and $V \subset Y$ such that $f(V) \subset U$. This data should satisfy the obvious compatibility condition: for any affine open subschemes $U^{\prime} \subset U$ and $V^{\prime} \subset V$ such that $f\left(V^{\prime}\right) \subset U^{\prime}$, the square diagram of CDG-ring morphisms between the CDG-rings $\mathcal{B}_{X}(U), \mathcal{B}_{X}\left(U^{\prime}\right)$, $\mathcal{B}_{Y}(V)$, and $\mathcal{B}_{Y}\left(V^{\prime}\right)$ must be commutative.

Let $\mathcal{B}_{X} \rightarrow \mathcal{B}_{Y}$ be a morphism of quasicoherent CDG-algebras compatible with a morphism of schemes $Y \rightarrow X$. Then for any quasicoherent left CDG-module $\mathcal{M}$ over $\mathcal{B}_{X}$, the quasicoherent graded left module $f^{*} \mathcal{M}=\mathcal{B}_{Y} \otimes_{f^{-1} \mathcal{B}_{X}} f^{-1} \mathcal{M}$ over $\mathcal{B}_{Y}$ has a natural structure of quasicoherent CDG-module over $\mathcal{B}_{Y}$. Similarly, for any quasicoherent left CDG-module $\mathcal{N}$ over $\mathcal{B}_{Y}$ the quasicoherent graded left module 
$f_{*} \mathcal{N}$ over $\mathcal{B}_{X}$ has a natural structure of quasicoherent CDG-module over $\mathcal{B}_{X}$. These CDG-module structures are defined in terms of the CDG-ring morphisms $\mathcal{B}_{X}(U) \rightarrow \mathcal{B}_{Y}(V)$. The above constructions provide the underived direct and inverse image functors, which can be viewed as triangulated functors $f^{*}: H^{0}\left(\mathcal{B}_{X^{-}}\right.$qcoh $) \rightarrow$ $H^{0}\left(\mathcal{B}_{Y^{-q}}\right.$ coh $)$ and $f_{*}: H^{0}\left(\mathcal{B}_{Y^{-q}}\right.$ qcoh $) \rightarrow H^{0}\left(\mathcal{B}_{X^{-q}}\right.$ coh $)$. The functor $f_{*}$ is right adjoint to the functor $f^{*}$.

The derived inverse image functor $\mathbb{L} f^{*}$ is in general only defined on CDG-modules satisfying certain finite flat dimension conditions. Restricting the functor $f^{*}$ to flat CDG-modules, we obtain a triangulated functor

$$
H^{0}\left(\mathcal{B}_{X}-\mathrm{qcoh}{ }_{\mathrm{fl}}\right) \longrightarrow H^{0}\left(\mathcal{B}_{Y}-\mathrm{q} \operatorname{coh}_{\mathrm{fl}}\right),
$$

which takes objects coacyclic with respect to $\mathcal{B}_{X}$-qcoh ${ }_{\mathrm{fl}}$ to objects coacyclic with respect to $\mathcal{B}_{Y}$-qcoh $\mathrm{fl}_{\mathrm{fl}}$ since the inverse image preserves infinite direct sums and short exact sequences of flat quasicoherent graded modules. Hence there is the induced triangulated functor $\mathrm{D}^{\mathrm{co}}\left(\mathcal{B}_{X}-\mathrm{q} \operatorname{coh}_{\mathrm{fl}}\right) \rightarrow \mathrm{D}^{\mathrm{co}}\left(\mathcal{B}_{Y}\right.$-qcoh $\left.{ }_{\mathrm{fl}}\right)$. Applying Theorem 1.4(a), we construct the derived inverse image functor

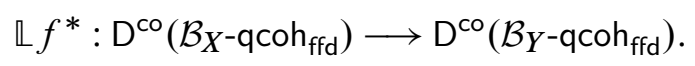

Assuming that there are enough vector bundles on $X$ and $Y$, and restricting the functor $f^{*}$ to flat coherent CDG-modules, we obtain a triangulated functor $H^{0}\left(\mathcal{B}_{X}-\operatorname{coh}_{\mathrm{fl}}\right) \rightarrow H^{0}\left(\mathcal{B}_{Y}\right.$-coh $\left.\operatorname{col}_{\mathrm{fl}}\right)$, which induces a triangulated functor

$$
\mathrm{D}^{\mathrm{abs}}\left(\mathcal{B}_{X}-\operatorname{coh}_{\mathrm{fl}}\right) \longrightarrow \mathrm{D}^{\mathrm{abs}}\left(\mathcal{B}_{Y}-\operatorname{coh}_{\mathrm{fl}}\right) \text {. }
$$

Assuming additionally that the quasicoherent graded algebras $\mathcal{B}_{X}$ and $\mathcal{B}_{Y}$ are Noetherian and applying Theorem 1.4(c), we construct the derived inverse image functor

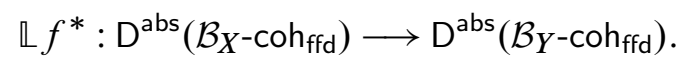

When $f$ is an affine morphism, the direct image of quasicoherent sheaves is an exact functor (preserving also infinite direct sums), so the functor $f_{*}: H^{0}\left(\mathcal{B}_{Y^{-}}\right.$qcoh) $\rightarrow$ $H^{0}\left(\mathcal{B}_{X^{-q}} \mathrm{qcoh}\right)$ induces a triangulated functor $\mathrm{D}^{\mathrm{co}}\left(\mathcal{B}_{Y^{-}} \mathrm{qcoh}\right) \rightarrow \mathrm{D}^{\mathrm{co}}\left(\mathcal{B}_{X^{-}}\right.$qcoh $)$. To construct the derived direct image functor between the coderived categories in the general case, we need to use injective resolutions.

From now on we assume that $\mathcal{B}_{X}$ and $\mathcal{B}_{Y}$ are Noetherian; so Lemma 1.7(b) is applicable to $\mathcal{B}_{Y}$. Restricting the functor $f_{*}$ to the full subcategory $H^{0}\left(\mathcal{B}_{Y}\right.$-qcoh $\left.{ }_{\text {inj }}\right) \subset$ $H^{0}\left(\mathcal{B}_{Y^{-q}} \mathrm{qcoh}\right)$ and composing it with the localization functor $H^{0}\left(\mathcal{B}_{X^{-}}\right.$qcoh $) \rightarrow$ $\mathrm{D}^{\mathrm{co}}\left(\mathcal{B}_{X}\right.$-qcoh $)$, we obtain the derived direct image functor

$$
\mathbb{R} f_{*}: \mathrm{D}^{\mathrm{co}}\left(\mathcal{B}_{Y} \text {-qcoh }\right) \longrightarrow \mathrm{D}^{\mathrm{co}}\left(\mathcal{B}_{X} \text {-qcoh }\right) .
$$


Proposition 1.8. Assume that there are enough vector bundles on $X$ and $Y$. Then the functors $\mathbb{L} f^{*}: \mathrm{D}^{\mathrm{abs}}\left(\mathcal{B}_{X}\right.$-coh $\left.{ }_{\mathrm{ffd}}\right) \rightarrow \mathrm{D}^{\mathrm{abs}}\left(\mathcal{B}_{Y}\right.$-coh $\left._{\mathrm{ffd}}\right)$ and $\mathbb{R} f_{*}: \mathrm{D}^{\mathrm{co}}\left(\mathcal{B}_{Y}\right.$-qcoh $) \rightarrow$ $\mathrm{D}^{\mathrm{co}}\left(\mathcal{B}_{X}\right.$-qcoh) are partially adjoint to each other in the following sense: for any objects $\mathcal{M} \in \mathrm{D}^{\mathrm{abs}}\left(\mathcal{B}_{X}\right.$-coh $\left.{ }_{\mathrm{ffd}}\right)$ and $\mathcal{N} \in \mathrm{D}^{\mathrm{co}}\left(\mathcal{B}_{Y}\right.$-qcoh $)$, there is a natural isomorphism of abelian groups

$$
\operatorname{Hom}_{D^{c o}\left(\mathcal{B}_{X} \text {-qcoh }\right)}\left(\iota_{X} \mathcal{M}, \mathbb{R} f_{*} \mathcal{N}\right) \simeq \operatorname{Hom}_{D^{c o}\left(\mathcal{B}_{Y} \text {-qcoh }\right)}\left(\iota_{Y} \mathbb{L} f^{*} \mathcal{M}, \mathcal{N}\right),
$$

where

$$
\begin{aligned}
& \iota_{X}: \mathrm{D}^{\mathrm{abs}}\left(\mathcal{B}_{X} \text {-coh }_{\mathrm{ffd}}\right) \longrightarrow \mathrm{D}^{\mathrm{co}}\left(\mathcal{B}_{X} \text {-qcoh }\right), \\
& \iota_{Y}: \mathrm{D}^{\mathrm{abs}}\left(\mathcal{B}_{X^{-}} \text {coh }_{\mathrm{ffd}}\right) \longrightarrow \mathrm{D}^{\mathrm{co}}\left(\mathcal{B}_{Y} \text {-qcoh }\right)
\end{aligned}
$$

are the natural fully faithful triangulated functors.

Proof. The functors $\iota_{X}$ and $\iota_{Y}$ are fully faithful by Theorem 1.4(c) and Proposition 1.5(b) and (d). Using Theorem 1.4(c), let us assume that $\mathcal{M} \in \mathrm{D}^{\text {abs }}\left(\mathcal{B}_{X}\right.$-coh $\left.{ }_{\mathrm{fl}}\right)$. We can also assume that $\mathcal{N} \in H^{0}\left(\mathcal{B}_{Y}\right.$-qcoh $\left.{ }_{\text {inj }}\right)$.

Then the left-hand side is the (filtered) inductive limit of

$$
\operatorname{Hom}_{H^{0}\left(\mathcal{B}_{X} \text {-qcoh }\right)}\left(\mathcal{M}^{\prime \prime}, f_{*} \mathcal{N}\right)
$$

over all morphisms $\mathcal{M}^{\prime \prime} \rightarrow \mathcal{M}$ in $H^{0}\left(\mathcal{B}_{X^{-}}\right.$qcoh) with a cone coacyclic with respect to $\mathcal{B}_{X}$-qcoh. According to the proofs of Proposition 1.5(b) and [Positselski 2011b, Theorem 3.11.1], any morphism from $\mathcal{M}$ to an object coacyclic with respect to $\mathcal{B}_{X}$-qcoh factorizes through an object absolutely acyclic with respect to $\mathcal{B}_{X}$-coh ${ }_{\mathrm{fl}}$. Thus the above inductive limit coincides with the similar limit taken over all morphisms $\mathcal{M}^{\prime} \rightarrow \mathcal{M}$ in $H^{0}\left(\mathcal{B}_{X}\right.$-coh $\left.{ }_{\mathrm{fl}}\right)$ with a cone absolutely acyclic with respect to $\mathcal{B}_{X}$-coh ${ }_{\mathrm{fl}}$.

By [loc. cit., Theorem 3.5(a), Remark 3.5, and Lemma 1.3], the right-hand side is isomorphic to $\operatorname{Hom}_{H^{0}\left(\mathcal{B}_{Y} \text {-qcoh }\right)}\left(f^{*} \mathcal{M}, \mathcal{N}\right)$ and to $\operatorname{Hom}_{H^{0}\left(\mathcal{B}_{Y} \text {-qcoh }\right)}\left(f^{*} \mathcal{M}^{\prime}, \mathcal{N}\right)$ since the objects of $H^{0}\left(\mathcal{B}_{Y}\right.$-qcoh inj $)$ are right orthogonal to any coacyclic objects in $H^{0}\left(\mathcal{B}_{Y}\right.$-qcoh). So the assertion follows from the adjointness of the functors $f^{*}$ and $f_{*}$ on the level of the homotopy categories of quasicoherent CDG-modules.

Remark 1.8. It is not immediately obvious from the above construction that the derived functor $\mathbb{R} f_{*}$ is compatible with the compositions; i.e., for $g: Z \rightarrow Y$ and $f: Y \rightarrow X$, one has $\mathbb{R}(f g)_{*} \simeq \mathbb{R} f_{*} \circ \mathbb{R} g_{*}$. The problem is that the direct image functor $f_{*}$ does not preserve injectivity of quasicoherent graded modules in general. When the derived direct image functors are adjoint to appropriately defined derived inverse images (see Section 1.9 below for some results of this kind), the problem reduces to checking that the derived inverse images are compatible with the compositions, which may be easier to see from our definitions.

One general approach to this problem is to replace injective quasicoherent graded $\mathcal{B}$-modules with quasicoherent graded $\mathcal{B}$-modules that are flabby as sheaves of 
graded abelian groups in our construction of the derived direct images. The class of flabby sheaves of abelian groups is closed under infinite direct sums since the underlying topological space of the scheme is Noetherian; it is also always closed under extensions and cokernels of injective morphisms. Whenever the quasicoherent graded algebra $\mathcal{B}$ is Noetherian, all injective quasicoherent graded $\mathcal{B}$-modules are flabby by Theorem A.3. Therefore, the coderived category of flabby quasicoherent CDG-modules over $\mathcal{B}$ is equivalent to the homotopy category $H^{0}\left(\mathcal{B}\right.$-qcoh $\left.{ }_{\text {inj }}\right)$ by a version of Lemma 1.7(b); hence it is also equivalent to the coderived category of all quasicoherent $C D G$-modules $\mathrm{D}^{\mathrm{co}}(\mathcal{B}$-qcoh) (cf. the proof of Proposition 1.7).

The direct images preserve exact triples of flabby sheaves, so derived direct images can be defined using flabby resolutions. The direct images also take flabby sheaves to flabby sheaves; hence the desired compatibility of their derived functors with the compositions of scheme morphisms follows.

Moreover, assuming additionally that the scheme has finite Krull dimension, the absolute derived category of flabby quasicoherent CDG-modules is equivalent to $\mathrm{D}^{\text {abs }}(\mathcal{B}$-qcoh) by a dual version of Theorem 1.4(b), as the "flabby dimension" of any quasicoherent graded $\mathcal{B}$-module is finite. This allows us to define the derived direct images on the absolute derived categories of quasicoherent CDG-modules (another approach to this question is to use the construction from the proof of Proposition 1.9 below). Notice that all our constructions of derived inverse images are also applicable to the categories $\mathrm{D}^{\text {abs }}(\mathcal{B}$-qcoh).

Finally, let us point out that for any morphism of quasicoherent CDG-algebras $\mathcal{B}_{X} \rightarrow \mathcal{B}_{Y}$ with Noetherian underlying quasicoherent graded algebras $\mathcal{B}_{X}$ and $\mathcal{B}_{Y}$ compatible with a morphism of separated Noetherian schemes $f: Y \rightarrow X$, the functor $\mathbb{R} f_{*}$ has a right adjoint functor

$$
f^{!}: \mathrm{D}^{\mathrm{co}}\left(\mathcal{B}_{X} \text {-qcoh }\right) \longrightarrow \mathrm{D}^{\mathrm{co}}\left(\mathcal{B}_{Y} \text {-qcoh }\right) .
$$

Indeed, the triangulated category $\mathrm{D}^{\mathrm{co}}\left(\mathcal{B}_{Y}\right.$-qcoh) is compactly generated by Proposition $1.5(\mathrm{~d})$, and the functor $\mathbb{R} f_{*}$ preserves infinite direct sums since the class of injective quasicoherent graded $\mathcal{B}_{Y}$-modules is closed under infinite direct sums, due to Noetherianness of $\mathcal{B}_{Y}$. So it remains to apply [Neeman 1996, Theorem 4.1].

There is a special situation when one can construct the above functor $f !$ explicitly. Assume that $f: Y \rightarrow X$ is an affine morphism. Let us say that the quasicoherent graded algebra $\mathcal{B}_{Y}$ is finite over $\mathcal{B}_{X}$ if for any affine open subscheme $U \subset X$, the graded $\mathcal{B}_{X}(U)$-module $\mathcal{B}_{Y}\left(f^{-1}(U)\right)$ is finitely generated, or in other words, if the quasicoherent graded $\mathcal{B}_{X}$-module $f_{*} \mathcal{B}_{Y}$ is coherent.

Let $\mathcal{B}_{X} \rightarrow \mathcal{B}_{Y}$ be a morphism of Noetherian quasicoherent CDG-algebras compatible with an affine morphism of separated Noetherian schemes $f: Y \rightarrow X$ such that the quasicoherent graded algebra $\mathcal{B}_{Y}$ is finite over $\mathcal{B}_{X}$. Given a quasicoherent graded left module $\mathcal{M}$ over $\mathcal{B}_{X}$, we set $\left(f^{!} \mathcal{M}\right)\left(f^{-1}(U)\right)$ to be the graded left module of 
homogeneous morphisms (of various degrees) $\operatorname{Hom}_{\mathcal{B}_{X}(U)}\left(\mathcal{B}_{Y}\left(f^{-1}(U)\right), \mathcal{M}(U)\right)$ over the graded ring $\mathcal{B}_{Y}\left(f^{-1}(U)\right)$ for any affine open subscheme $U \subset X$. Due to the finiteness condition on $\mathcal{B}_{Y}$ over $\mathcal{B}_{X}$, for any affine open subscheme $V \subset U$, there are natural isomorphisms $\left(f^{!} \mathcal{M}\right)\left(f^{-1}(V)\right) \simeq \mathcal{O}_{X}(V) \otimes_{\mathcal{O}_{X}(U)}\left(f^{!} \mathcal{M}\right)\left(f^{-1}(U)\right) \simeq$ $\mathcal{O}_{Y}\left(f^{-1}(V)\right) \otimes_{\mathcal{O}_{Y}\left(f^{-1}(U)\right)}\left(f^{!} \mathcal{M}\right)\left(f^{-1}(U)\right)$, which allow us to extend the assignment $f^{-1}(U) \longmapsto\left(f^{!} \mathcal{M}\right)\left(f^{-1}(U)\right)$ to a quasicoherent graded module $f^{!}(\mathcal{M})$ over the quasicoherent graded algebra $\mathcal{B}_{Y}$.

Given a quasicoherent CDG-module $\mathcal{M}$ over $\mathcal{B}_{X}$, the conventional rule

$$
d(g)(m)=d(g(m))-(-1)^{|g|} g(d(m))
$$

(with the usual change-of-connection modifications) defines the structure of a quasicoherent CDG-module over $\mathcal{B}_{Y}$ on the quasicoherent graded module $f^{!}(\mathcal{M})$. This construction provides a triangulated functor $f^{!}: H^{0}\left(\mathcal{B}_{X^{-}}\right.$qcoh $) \rightarrow H^{0}\left(\mathcal{B}_{Y^{-q}}\right.$-qcoh $)$ right adjoint to the triangulated functor $f_{*}: H^{0}\left(\mathcal{B}_{Y-q c o h}\right) \rightarrow H^{0}\left(\mathcal{B}_{X}\right.$-qcoh $)$. Restricting the functor $f^{!}: H^{0}\left(\mathcal{B}_{X}\right.$-qcoh $) \rightarrow H^{0}\left(\mathcal{B}_{Y}\right.$-qcoh $)$ to the full subcategory of injective quasicoherent CDG-modules in $H^{0}\left(\mathcal{B}_{X}\right.$-qcoh) and taking into account Lemma 1.7(b), we obtain the right derived functor

$$
\mathbb{R} f^{!}: \mathrm{D}^{\mathrm{co}}\left(\mathcal{B}_{X^{-}} \text {qcoh }\right) \longrightarrow \mathrm{D}^{\mathrm{co}}\left(\mathcal{B}_{Y} \text {-qcoh }\right),
$$

which is right adjoint to the (underived, as the morphism $f$ is affine) direct image functor $f_{*}: \mathrm{D}^{\mathrm{co}}\left(\mathcal{B}_{Y}\right.$-qcoh $) \rightarrow \mathrm{D}^{\mathrm{co}}\left(\mathcal{B}_{X}\right.$-qcoh $)$. In other words, the functor $\mathbb{R} f^{!}$ coincides with the above adjoint functor $f^{!}: \mathrm{D}^{\mathrm{co}}\left(\mathcal{B}_{X^{-}} \mathrm{qcoh}\right) \rightarrow \mathrm{D}^{\mathrm{co}}\left(\mathcal{B}_{Y^{-}}\right.$qcoh $)$in our special case.

1.9. Morphisms of finite flat dimension. Let $f: Y \rightarrow X$ be a morphism of separated Noetherian schemes, and let $\mathcal{B}_{X} \rightarrow \mathcal{B}_{Y}$ be a compatible morphism of quasicoherent CDG-algebras. We will say that the quasicoherent graded algebra $\mathcal{B}_{Y}$ has finite flat dimension over $\mathcal{B}_{X}$ if (the left derived functor of) the functor of inverse image $f^{*}$ acting between the abelian categories of quasicoherent graded modules over $\mathcal{B}_{X}$ and $\mathcal{B}_{Y}$ has finite homological dimension. Equivalently, for any affine open subschemes $U \subset X$ and $V \subset Y$ such that $f(V) \subset U$, the graded right $\mathcal{B}_{X}(U)$-module $\mathcal{B}_{Y}(V)$ should have finite flat dimension.

A quasicoherent graded $\mathcal{B}_{X}$-module is said to be adjusted to $f^{*}$ if its derived inverse image under $f$, as an object of the derived category of the abelian category of quasicoherent graded $\mathcal{B}_{Y}$-modules, coincides with the underived inverse image. Denote the DG-category of quasicoherent CDG-modules over $\mathcal{B}_{X}$ whose underlying graded $\mathcal{B}_{X}$-modules are adjusted to $f^{*}$ by $\mathcal{B}_{X}$-qcoh $f$-adj. When $\mathcal{B}_{X}$ is Noetherian, let $\mathcal{B}_{X}$-coh ${ }_{\text {-adj }}$ denote the similarly defined DG-category of coherent CDG-modules. We will use our usual notation for the absolute derived and coderived categories of these DG-categories of CDG-modules. 
Lemma 1.9. Assume that the quasicoherent graded algebra $\mathcal{B}_{Y}$ has finite flat dimension over $\mathcal{B}_{X}$. Then

(a) the functor $\mathrm{D}^{\mathrm{co}}\left(\mathcal{B}_{X}\right.$-qcoh $\left.{ }_{\text {-adj }}\right) \rightarrow \mathrm{D}^{\mathrm{co}}\left(\mathcal{B}_{X}\right.$-qcoh $)$ induced by the embedding of DG-categories $\mathcal{B}_{X}$-qcoh ${ }_{\text {-adj }} \rightarrow \mathcal{B}_{X}$-qcoh is an equivalence of triangulated categories;

(b) the functor $\mathrm{D}^{\mathrm{abs}}\left(\mathcal{B}_{X}\right.$-qcoh $\left.{ }_{f \text {-adj }}\right) \rightarrow \mathrm{D}^{\mathrm{abs}}\left(\mathcal{B}_{X}\right.$-qcoh) induced by the embedding of DG-categories $\mathcal{B}_{X}$-qcoh ${ }_{\text {-adj }} \rightarrow \mathcal{B}_{X}$-qcoh is an equivalence of triangulated categories;

(c) if there are enough vector bundles on $X$ and $\mathcal{B}_{X}$ is Noetherian, the functor $\mathrm{D}^{\mathrm{abs}}\left(\mathcal{B}_{X}\right.$-coh $\left.f_{\text {-adj }}\right) \rightarrow \mathrm{D}^{\mathrm{abs}}\left(\mathcal{B}_{X}\right.$-coh $)$ induced by the embedding of DG-categories $\mathcal{B}_{X}$-coh $\operatorname{codj}_{\text {-adj }} \rightarrow \mathcal{B}_{X}$-coh is an equivalence of triangulated categories.

Proof. This is a version of Theorem 1.4, provable in the same way (cf. Corollary 2.6 below). The assertions hold, because any quasicoherent graded $\mathcal{B}_{X}$-module has a finite left resolution consisting of quasicoherent CDG-modules adjusted to $f^{*}$, and it is similar for coherent CDG-modules.

The functor of inverse image $f^{*}: H^{0}\left(\mathcal{B}_{X^{-q}}\right.$ coh $) \rightarrow H^{0}\left(\mathcal{B}_{Y}\right.$-qcoh $)$ takes CDGmodules coacyclic with respect to $\mathcal{B}_{X}$-qcoh $f_{\text {-adj }}$ to CDG-modules coacyclic with respect to $\mathcal{B}_{Y}$-qcoh, and hence induces a triangulated functor $\mathrm{D}^{\mathrm{co}}\left(\mathcal{B}_{X^{-}}\right.$-qcoh $\left.{ }_{f \text {-adj }}\right) \rightarrow$ $\mathrm{D}^{\mathrm{co}}\left(\mathcal{B}_{Y}\right.$-qcoh). Taking Lemma 1.9 into account, we construct the derived inverse image functor

$$
\llbracket f^{*}: \mathrm{D}^{\mathrm{co}}\left(\mathcal{B}_{X^{-}} \mathrm{qcoh}\right) \longrightarrow \mathrm{D}^{\mathrm{co}}\left(\mathcal{B}_{Y} \text {-qcoh }\right) .
$$

One shows that this functor is left adjoint to the functor $\mathbb{R} f_{*}$ constructed in Section 1.8 in the way analogous to (but simpler than) the proof of Proposition 1.8.

When there are enough vector bundles on $X$, and $\mathcal{B}_{X}$ and $\mathcal{B}_{Y}$ are Noetherian, we construct the derived inverse image functor

$$
\mathbb{L} f^{*}: \mathrm{D}^{\mathrm{abs}}\left(\mathcal{B}_{X^{-}} \text {coh }\right) \longrightarrow \mathrm{D}^{\mathrm{abs}}\left(\mathcal{B}_{Y^{-}}-\mathrm{coh}\right)
$$

in a similar way.

Let $\mathcal{B}_{X}^{\text {op }}$ and $\mathcal{B}_{Y}^{\text {op }}$ denote the quasicoherent graded algebras with the opposite multiplication to $\mathcal{B}_{X}$ and $\mathcal{B}_{Y}$.

Proposition 1.9. When $\mathcal{B}_{Y}^{\mathrm{op}}$ has finite flat dimension over $\mathcal{B}_{X}^{\mathrm{op}}$, the derived inverse image functor $\mathbb{L} f^{*}: \mathrm{D}^{\mathrm{co}}\left(\mathcal{B}_{X}\right.$-qcoh $\left.{ }_{\mathrm{ffd}}\right) \rightarrow \mathrm{D}^{\mathrm{co}}\left(\mathcal{B}_{Y}\right.$-qcoh $\left.{ }_{\mathrm{ffd}}\right)$ constructed in Section 1.8 has a right adjoint functor

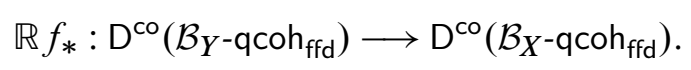


Proof. Let $\left\{U_{\alpha}\right\}$ be a finite affine covering of $Y$. To any object $\mathcal{N} \in \mathcal{B}_{Y \text {-qcoh }}$ ffd, assign the total CDG-module $\mathbb{R}_{\left\{U_{\alpha}\right\}} f_{*} \mathcal{N}$ of the finite Čech complex

$$
\left.\left.\bigoplus_{\alpha} f\right|_{U_{\alpha} *}\left(\left.\mathcal{N}\right|_{U_{\alpha}}\right) \longrightarrow \bigoplus_{\alpha<\beta} f\right|_{U_{\alpha} \cap \mathcal{U}_{\beta} *}\left(\left.\mathcal{N}\right|_{U_{\alpha} \cap U_{\beta}}\right) \longrightarrow \cdots
$$

of CDG-modules over $\mathcal{B}_{X}$.

The terms of this complex belong to $\mathcal{B}_{X}$-qcoh ${ }_{\text {ffd }}$ since the morphism $\left.f\right|_{V}: V \rightarrow X$ is affine for any intersection $V$ of a nonempty subset of affine open subschemes $U_{\alpha} \subset Y$ and the quasicoherent graded algebra $\mathcal{B}_{Y}^{\text {op }}$ has finite flat dimension over $\mathcal{B}_{X}^{\text {op }}$. Hence one has $\mathbb{R}_{\left\{U_{\alpha}\right\}} f_{*} \mathcal{N} \in \mathcal{B}_{X}$-qcoh ffd $_{\text {ff }}$; it is clear that $\mathbb{R}_{\left\{U_{\alpha}\right\}} f_{*}$ is a DG-functor $\mathcal{B}_{Y}$-qcoh ${ }_{\text {ffd }} \rightarrow \mathcal{B}_{X}$-qcoh ${ }_{\text {ffd }}$ taking coacyclic objects to coacyclic objects. So we have the induced functor $\mathbb{R} f_{*}$ between the coderived categories.

It remains to obtain the adjunction isomorphism

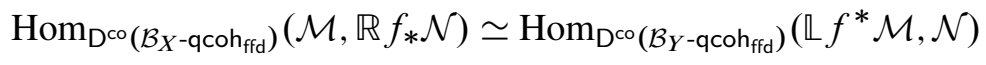

for $\mathcal{M} \in \mathrm{D}^{\mathrm{co}}\left(\mathcal{B}_{X}\right.$-qcoh $\left.{ }_{\mathrm{ffd}}\right)$. Denote by $\mathcal{N}_{+}$the total CDG-module of the finite complex

$$
C_{\left\{U_{\alpha}\right\}}^{\bullet} \mathcal{N}=\left(\bigoplus_{\alpha} j_{U_{\alpha} *} j_{U_{\alpha}}^{*} \mathcal{N} \longrightarrow \bigoplus_{\alpha<\beta} j_{U_{\alpha} \cap \mathcal{U}_{\beta} *} j_{U_{\alpha} \cap U_{\beta}}^{*} \mathcal{N} \longrightarrow \cdots\right)
$$

of CDG-modules over $\mathcal{B}_{Y}$ (where $j_{V}: V \rightarrow Y$ denotes the embedding of an affine open subscheme). Then we have $\mathbb{R}_{\left\{U_{\alpha}\right\}} f_{*} \mathcal{N} \simeq f_{*} \mathcal{N}_{+}$. There is a natural closed morphism $\mathcal{N} \rightarrow \mathcal{N}_{+}$of CDG-modules over $\mathcal{B}_{Y}$ with the cone coacyclic (and even absolutely acyclic) with respect to $\mathcal{B}_{Y}$-qcoh ${ }_{\text {ffd }}$.

For any CDG-module $\mathcal{Q} \in \mathcal{B}_{Y-q}$ coh $_{\text {ffd }}$ such that $f_{*} \mathcal{Q} \in \mathcal{B}_{X}$-qcoh ${ }_{\text {ffd }}$, there is a natural map

$$
\left.\psi: \operatorname{Hom}_{\mathrm{D}^{\mathrm{co}}\left(\mathcal{B}_{X}\right. \text {-qcoh }}(\mathcal{H f d}) f_{*} \mathcal{Q}\right) \longrightarrow \operatorname{Hom}_{\mathrm{D}^{\mathrm{co}}\left(\mathcal{B}_{Y-\mathrm{qcoh}} \mathrm{ffd}\right)}\left(\mathbb{L} f^{*} \mathcal{M}, \mathcal{Q}\right) .
$$

Indeed, by the proof of Theorem 1.4(a), any morphism $\mathcal{M} \rightarrow f_{*} \mathcal{Q}$ in $\mathrm{D}^{\mathrm{co}}\left(\mathcal{B}_{X}\right.$-qcoh $\left.{ }_{\mathrm{ffd}}\right)$ can be represented as a fraction formed by a morphism $\mathcal{M}^{\prime} \rightarrow \mathcal{M}$ in $H^{0}\left(\mathcal{B}_{X^{-}} \mathrm{qcoh}_{\mathrm{ffd}}\right)$, with $\mathcal{M}^{\prime} \in \mathcal{B}_{X}$-qcoh ${ }_{\mathrm{fl}}$ and a cone coacyclic with respect to $\mathcal{B}_{X}$-qcoh ${ }_{\mathrm{ffd}}$, and a morphism $\mathcal{M}^{\prime} \rightarrow f_{*} \mathcal{Q}$ in $H^{0}\left(\mathcal{B}_{X^{-q}}\right.$ coh $\left._{\text {ffd }}\right)$. To such a fraction, the map $\psi$ assigns the related morphism $\mathbb{L} f^{*} \mathcal{M}=f^{*} \mathcal{M}^{\prime} \rightarrow \mathcal{Q}$.

For a fixed $\mathcal{M}$, the map $\psi$ is a morphism of cohomological functors of the

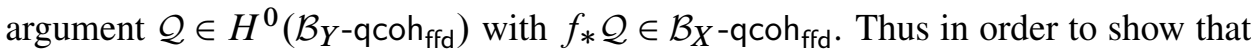
it is an isomorphism for $\mathcal{Q}=\mathcal{N}_{+}$, it suffices to check that it is an isomorphism for $\mathcal{Q}=j_{V *} \mathcal{P}$ for every affine $V \subset Y$ and $\left.\mathcal{P} \in \mathcal{B}_{Y}\right|_{V^{-}}$qcoh $_{\text {ffd }}$. This follows from the adjunction isomorphism

$$
\operatorname{Hom}_{D^{c o}\left(\mathcal{B}_{X} \text {-qcoh }_{\text {ffd }}\right)}\left(\mathcal{M},\left.f\right|_{V *} \mathcal{P}\right) \simeq \operatorname{Hom}_{D^{c o}\left(\left.\mathcal{B}_{Y}\right|_{V^{-q}} \operatorname{qcoh}_{\text {ffd }}\right)}\left(\left.\mathbb{L} f\right|_{V} ^{*} \mathcal{M}, \mathcal{P}\right)
$$


and the similar isomorphism for the embedding $j_{V}$, which hold because the functors $\left.f\right|_{V *}$ and $j_{V *}$ are exact, the morphisms $\left.f\right|_{V}$ and $j_{V}$ being affine.

Remark 1.9. One can also use the above Čech complex approach in order to construct a version of the derived functor $\mathbb{R} f_{*}: \mathrm{D}^{\mathrm{co}}\left(\mathcal{B}_{Y}\right.$-qcoh $) \rightarrow \mathrm{D}^{\mathrm{co}}\left(\mathcal{B}_{X}\right.$-qcoh $)$. One can check that this construction agrees with the injective resolution construction from Section 1.8, using the fact that the restrictions of injective quasicoherent graded $\mathcal{B}_{Y}$-modules to open subschemes are injective (Theorem A.3). Alternatively, in the assumption of finite flat dimension of $\mathcal{B}_{Y}$ over $\mathcal{B}_{X}$, one checks that both constructions provide functors right adjoint to $\mathbb{L} f^{*}$, hence they are isomorphic.

This allows us to conclude that the derived functors $\mathbb{R} f_{*}$ acting on arbitrary quasicoherent CDG-modules and quasicoherent CDG-modules of finite flat dimension form a commutative diagram with the natural functors from the coderived categories of the latter to the coderived categories of the former.

1.10. Supports of CDG-modules. Let $X$ be a Noetherian scheme. The set-theoretic support of a quasicoherent sheaf $\mathcal{M}$ on $X$ is the minimal closed subset $T \subset X$ such that the restriction of $\mathcal{M}$ to the open subscheme $X \backslash T$ vanishes. Given a Noetherian quasicoherent graded algebra $\mathcal{B}$ over $X$ and a quasicoherent graded $\mathcal{B}$-module $\mathcal{M}$, the set-theoretic support $T=\operatorname{Supp} \mathcal{M}$ of $\mathcal{M}$ is defined similarly. It only depends on the underlying quasicoherent $\mathcal{O}_{X}$-module of $\mathcal{M}$.

Let $\mathcal{B}$ be a quasicoherent CDG-algebra over $X$ whose underlying quasicoherent graded algebra $\mathcal{B}$ is Noetherian. Fix a closed subset $T \subset X$. Denote by $\mathcal{B}-q c o h_{T}$ the full DG-subcategory in $\mathcal{B}$-qcoh consisting of all the quasicoherent CDG-modules whose underlying quasicoherent graded $\mathcal{B}$-modules have their set-theoretic supports contained in $T$. The DG-category $\mathcal{B}$-coh $T$ of coherent CDG-modules with settheoretic support in $T$ is defined similarly.

Let $\mathrm{D}^{\mathrm{co}}(\mathcal{B}$-qcoh $T)$ and $\mathrm{D}^{\mathrm{abs}}\left(\mathcal{B}\right.$-coh $\left.{ }_{T}\right)$ denote the coderived and the absolute derived category of these DG-categories of CDG-modules. Finally, let $\mathcal{B}$-qcoh ${ }_{T \text {,inj }}$ denote the DG-category of quasicoherent CDG-modules over $\mathcal{B}$ whose underlying quasicoherent graded modules are injective objects of the abelian category of quasicoherent graded $\mathcal{B}$-modules with set-theoretic support contained in $T$.

Proposition 1.10. (a) The functor $H^{0}\left(\mathcal{B}\right.$-qcoh $\left.{ }_{T, \text { inj }}\right) \rightarrow \mathrm{D}^{\mathrm{co}}(\mathcal{B}$-qcoh $T)$ induced by the embedding of $D G$-categories $\mathcal{B}$-qcoh ${ }_{T \text {,inj }} \rightarrow \mathcal{B}$-qcoh ${ }_{T}$ is an equivalence of triangulated categories.

(b) The functor $\mathrm{D}^{\mathrm{abs}}\left(\mathcal{B}-\mathrm{coh}_{T}\right) \rightarrow \mathrm{D}^{\mathrm{co}}(\mathcal{B}-\mathrm{qcoh} T)$ induced by the embedding of $D G$-categories $\mathcal{B}$-coh $\rightarrow \mathcal{B}$-qcoh ${ }_{T}$ is fully faithful and its image is a set of compact generators of the target category.

(c) The functor $\mathrm{D}^{\mathrm{co}}(\mathcal{B}-\mathrm{qcoh} T) \rightarrow \mathrm{D}^{\mathrm{co}}(\mathcal{B}$-qcoh) induced by the embedding of $D G$-categories $\mathcal{B}$-qcoh ${ }_{T} \rightarrow \mathcal{B}$-qcoh is fully faithful. 
(d) The functor $\mathrm{D}^{\mathrm{abs}}(\mathcal{B}$-coh $T) \rightarrow \mathrm{D}^{\text {abs }}(\mathcal{B}$-coh) induced by the embedding of $D G$ categories $\mathcal{B}$ - $\operatorname{coh}_{T} \rightarrow \mathcal{B}$-coh is fully faithful.

Proof. Part (a) is essentially a particular case of [Positselski 2011b, Theorem and Remark in Section 3.7]. It is only important here that there are enough injective objects in the abelian category of quasicoherent graded $\mathcal{B}$-modules supported settheoretically in $T$ and the class of such injective objects is closed under infinite direct sums. This is so because the abelian category in question is a locally Noetherian Grothendieck category (since $X$ and $\mathcal{B}$ are Noetherian). Part (b) can be proven in the same way as the results of [loc. cit., Section 3.11]. Part (d) follows from parts (b) and (c) and Proposition 1.5(d).

Finally, part (c) follows from part (a), Lemma 1.7(b), and the fact that any injective object $\mathcal{J}$ in the category of quasicoherent graded $\mathcal{B}$-modules supported set-theoretically in $T$ is also an injective object in the category of arbitrary quasicoherent graded $\mathcal{B}$-modules. The latter is essentially a reformulation of the Artin-Rees lemma.

Indeed, it suffices to check that for any coherent graded $\mathcal{B}$-module $\mathcal{M}$ and its coherent graded $\mathcal{B}$-submodule $\mathcal{N}$, any morphism of quasicoherent graded $\mathcal{B}$-modules $\phi: \mathcal{N} \rightarrow \mathcal{J}$ can be extended to $\mathcal{M}$. Let $Z$ be a closed subscheme structure on the closed subset $T \subset X$. Then there is an integer $n \geq 0$ such that the morphism $\phi$ annihilates $\mathcal{I}_{Z}^{n} \mathcal{N}$ (where $\mathcal{I}_{Z}$ is the sheaf of ideals of the closed subscheme $Z$ ). By Lemma A.3, there exists $m \geq 0$ such that $\mathcal{I}_{Z}^{m} \mathcal{M} \cap \mathcal{N} \subset \mathcal{I}_{Z}^{n} \mathcal{N}$. Then there exists a morphism $\mathcal{M} / \mathcal{I}_{Z}^{m} \mathcal{M} \rightarrow \mathcal{J}$ of quasicoherent graded $\mathcal{B}$-modules supported settheoretically in $T$ which extends the given morphism into $\mathcal{J}$ from the quasicoherent graded $\mathcal{B}$-submodule $\mathcal{N} /\left(\mathcal{I}_{Z}^{m} \mathcal{M} \cap \mathcal{N}\right) \subset \mathcal{M} / \mathcal{I}_{Z}^{m} \mathcal{M}$.

Let $U \subset X$ denote the open subscheme $X \backslash T$.

Theorem 1.10. (a) The functor of restriction to the open subscheme $D^{\mathrm{co}}(\mathcal{B}$-qcoh) $\rightarrow$ $\mathrm{D}^{\mathrm{co}}\left(\left.\mathcal{B}\right|_{U}\right.$-qcoh) is the Verdier localization functor by the thick subcategory

$$
\mathrm{D}^{\mathrm{co}}(\mathcal{B}-\mathrm{qcoh} T) \subset \mathrm{D}^{\mathrm{co}}(\mathcal{B}-\mathrm{qcoh}) .
$$

In particular, the kernel of the restriction functor coincides with the subcategory $\mathrm{D}^{\mathrm{co}}(\mathcal{B}-\mathrm{qcoh} T)$.

(b) The functor of restriction to the open subscheme $\mathrm{D}^{\mathrm{abs}}(\mathcal{B}$-coh $) \rightarrow \mathrm{D}^{\mathrm{abs}}\left(\left.\mathcal{B}\right|_{U}\right.$-coh) is the Verdier localization functor by the triangulated subcategory

$$
\mathrm{D}^{\mathrm{abs}}(\mathcal{B} \text {-coh } T) \subset \mathrm{D}^{\mathrm{abs}}(\mathcal{B} \text {-coh }) \text {. }
$$

In particular, the kernel of the restriction functor coincides with the thick envelope of (i.e., the minimal thick subcategory containing) $\mathrm{D}^{\mathrm{abs}}\left(\mathcal{B}-\operatorname{coh}_{T}\right)$ in $\mathrm{D}^{\mathrm{abs}}(\mathcal{B}$-coh). 
Proof. Let $j: U \rightarrow X$ denote the natural open embedding. To prove part (a), consider the functor $\mathbb{R} j_{*}: \mathrm{D}^{\mathrm{co}}\left(\left.\mathcal{B}\right|_{U}\right.$-qcoh $) \rightarrow \mathrm{D}^{\mathrm{co}}(\mathcal{B}$-qcoh) as constructed in Section 1.8. Since the quasicoherent graded algebra $\left.\mathcal{B}\right|_{U}$ is flat over $\mathcal{B}$, the functor $\mathbb{R} j_{*}$ is right adjoint to the restriction functor $j^{*}: \mathrm{D}^{\mathrm{co}}(\mathcal{B}$-qcoh $) \rightarrow \mathrm{D}^{\mathrm{co}}\left(\left.\mathcal{B}\right|_{U}\right.$-qcoh $)$. Obviously, the composition $j^{*} \mathbb{R} j_{*}$ is the identity functor. It follows that the functor $j^{*}$ is a Verdier localization functor by its kernel, which is the full subcategory consisting of all the cones of the adjunction morphisms $\mathcal{M} \rightarrow \mathbb{R} j_{*} j^{*} \mathcal{M}$, where $\mathcal{M} \in \mathrm{D}^{\mathrm{co}}(\mathcal{B}$-qcoh).

Represent the object $\mathcal{M}$ by a CDG-module with an injective underlying quasicoherent graded $\mathcal{B}$-module. By Theorem A.3, the quasicoherent graded $\left.\mathcal{B}\right|_{U}$-module $j^{*} \mathcal{M}$ is then also injective, so we have $\mathbb{R} j_{*} j^{*} \mathcal{M}=j_{*} j^{*} \mathcal{M}$. Obviously, both the kernel and the cokernel of the closed morphism of CDG-modules $\mathcal{M} \rightarrow j_{*} j^{*} \mathcal{M}$ belong to $\mathcal{B}$-qcoh ${ }_{T}$, and it follows, in view of Proposition 1.10(c), that the cone also belongs to $\mathrm{D}^{\mathrm{co}}(\mathcal{B}-\mathrm{qcoh} T)$.

To prove part (b), notice first that any coherent CDG-module over $\left.\mathcal{B}\right|_{U}$ can be extended to a coherent CDG-module over $\mathcal{B}$ (because a coherent sheaf $\mathcal{K}$ on $U$ can be extended to a coherent subsheaf of $j_{*} \mathcal{K}$ ), so the restriction functor is essentially surjective. Taking this observation into account, part (b) follows from part (a), Proposition 1.10(b), Proposition 1.5(d), and the standard results about localization of compactly generated triangulated categories [Neeman 1992, Lemma 2.5 to Theorem 2.1].

Define the category-theoretic support $\operatorname{supp} \mathcal{M}$ of a quasicoherent CDG-module $\mathcal{M}$ over $\mathcal{B}$ as the minimal closed subset $T \subset X$ such that the restriction $\left.\mathcal{M}\right|_{U}$ of $\mathcal{M}$ to the open subscheme $U=X \backslash T$ is a coacyclic CDG-module over $\left.\mathcal{B}\right|_{U}$. In other words, $X \backslash \operatorname{supp} \mathcal{M}$ is the union of all open subschemes $V \subset X$ such that $\left.\mathcal{M}\right|_{V}$ is a coacyclic CDG-module over $\left.\mathcal{B}\right|_{V}$ (see Remark 1.3). Obviously, one has $\operatorname{supp} \mathcal{M} \subset \operatorname{Supp} \mathcal{M}$.

The category-theoretic support of a coherent CDG-module $\mathcal{M}$ over $\mathcal{B}$ can be equivalently defined as the minimal closed subset $T \subset X$ such that the restriction $\left.\mathcal{M}\right|_{U}$ of $\mathcal{M}$ to the open subscheme $U=X \backslash T$ is absolutely acyclic. Indeed, any CDG-module from $\left.\mathcal{B}\right|_{U}$-coh that is coacyclic with respect to $\left.\mathcal{B}\right|_{U}$-qcoh is also absolutely acyclic with respect to $\left.\mathcal{B}\right|_{U}$-coh by Proposition (d).

Corollary 1.10. (a) For any quasicoherent $C D G$-module $\mathcal{M}$ over $\mathcal{B}$ with categorytheoretic support $\operatorname{supp} \mathcal{M}$ contained in $T$, there exists a quasicoherent $C D G$ module $\mathcal{M}^{\prime}$ over $\mathcal{B}$ such that $\mathcal{M}$ is isomorphic to $\mathcal{M}^{\prime}$ in $\mathrm{D}^{\mathrm{co}}(\mathcal{B}$-qcoh) and set-theoretic support Supp $\mathcal{M}^{\prime}$ is contained in $T$.

(b) For any coherent $C D G$-module $\mathcal{M}$ over $\mathcal{B}$ with category-theoretic support supp $\mathcal{M}$ contained in $T$, there exists a coherent $C D G$-module $\mathcal{M}^{\prime}$ over $\mathcal{B}$ such that $\mathcal{M}$ is isomorphic to a direct summand of $\mathcal{M}^{\prime}$ in $\mathrm{D}^{\mathrm{abs}}(\mathcal{B}$-coh) and set-theoretic support Supp $\mathcal{M}^{\prime}$ is contained in $T$. 
Proof. Follows immediately from Theorem 1.10.

Remark 1.10. One can prove that the restriction functor in Theorem 1.10(a) is a Verdier localization functor without assuming the quasicoherent graded algebra $\mathcal{B}$ to be Noetherian. Indeed, one can construct a right adjoint functor $\mathbb{R} j_{*}$ to the restriction functor $j^{*}$ in the way similar to that of Proposition 1.9; then it is easy to see that $j^{*} \mathbb{R} j_{*}$ is the identity functor.

When $\mathcal{B}$ is Noetherian, Theorem 1.10 can be generalized as follows. Let $S$ and $T$ be closed subsets in $X$; set $U=X \backslash T$. Then the restriction functor $\mathrm{D}^{\mathrm{co}}\left(\mathcal{B}\right.$-qcoh $\left.{ }_{S}\right) \rightarrow$

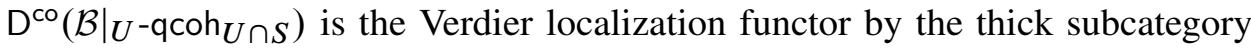

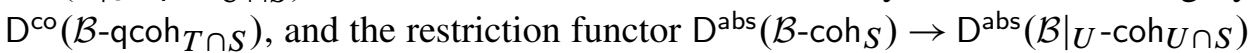
is the Verdier localization functor by the triangulated subcategory $\mathrm{D}^{\text {abs }}\left(\mathcal{B}-\operatorname{coh}_{T \cap S}\right)$. The proof is similar to the above.

It is not difficult to deduce from the latter assertions, using the result of [Neeman 1996, Theorem 2.1(5)], that the property of an object of $D^{\mathrm{co}}(\mathcal{B}$-qcoh) to belong to the thick envelope of $\mathrm{D}^{\text {abs }}(\mathcal{B}$-coh) is local in $X$. Using the Čech exact sequence as in Remark 1.3, one can easily see that the property of an object of $D^{\text {abs }}(\mathcal{B}$-qcoh) to belong to $D^{\text {abs }}\left(\mathcal{B}\right.$-qcoh $\left.{ }_{\mathrm{fl}}\right)$ is also local.

We do not know whether the property of an object of $D^{\text {abs }}\left(\mathcal{B}\right.$-coh) or $D^{\text {abs }}\left(\mathcal{B}\right.$-qcoh fl $\left._{f}\right)$ to belong to $D^{\text {abs }}\left(\mathcal{B}\right.$-coh $\left.{ }_{f l}\right)$ is local in general. In the particular case of matrix factorizations, such results will be proven in Section 3.2 using the connection with singularity categories (cf. Remark 3.6).

Notice that the theory of localization for compactly generated triangulated categories, on which the proof of Theorem 1.10(b) is based, was originally applied in algebraic geometry for the purposes of the Thomason-Trobaugh localization theory of perfect complexes. In this section we apply it to coherent CDG-modules. In fact, we will see in Section 3.3 that the localization theory fails for locally free matrix factorizations of finite rank.

\section{Triangulated categories of relative singularities}

2.1. Relative singularity category. Recall that $X$ denotes a separated Noetherian scheme with enough vector bundles. The triangulated category of singularities $\mathrm{D}_{\text {Sing }}^{\mathrm{b}}(X)$ of the scheme $X$ is defined [Orlov 2004, Section 1.2] as the quotient category of the bounded derived category $\mathrm{D}^{\mathrm{b}}(X$-coh) of coherent sheaves on $X$ by its thick subcategory $\operatorname{Perf}(X)$ of perfect complexes on $X$.

The perfect complexes, in our assumptions, can be simply defined as bounded complexes of locally free sheaves of finite rank, so $\operatorname{Perf}(X)=\mathrm{D}^{\mathrm{b}}\left(X\right.$-coh $\left.\mathrm{cof}_{\mathrm{ff}}\right)$ is the

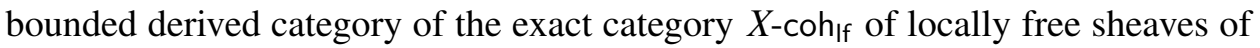
finite rank on $X$. Equivalently, the perfect complexes are the compact objects of the 
unbounded derived category of quasicoherent sheaves $\mathrm{D}(X$-qcoh) on the scheme $X$ [Neeman 1996, Examples 1.10-1.11 and Corollary 2.3].

Let $Z \subset X$ be a closed subscheme such that $\mathcal{O}_{Z}$ has finite flat dimension as an $\mathcal{O}_{X}$-module. In this case the derived inverse image functor $\llbracket i^{*}$ for the closed embedding $i: Z \rightarrow X$ acts on the bounded derived categories of coherent sheaves, $\mathrm{D}^{\mathrm{b}}(X$-coh $) \rightarrow \mathrm{D}^{\mathrm{b}}(Z$-coh $)$. We call the quotient category of $\mathrm{D}^{\mathrm{b}}(Z$-coh $)$ by the thick subcategory generated by the objects in the image of this functor the triangulated category of singularities of $Z$ relative to $X$ and denote it by $D_{\text {Sing }}^{b}(Z / X)$.

Note that the triangulated category of relative singularities $D_{\text {Sing }}^{b}(Z / X)$ is a quotient category of the conventional (absolute) triangulated category of singularities $\mathrm{D}_{\text {Sing }}^{\mathrm{b}}(Z)$ of the scheme $Z$. Indeed, the thick subcategory $\operatorname{Perf}(Z) \subset \mathrm{D}^{\mathrm{b}}(Z$-coh $)$ is generated by any ample family of vector bundles on $Z$ since any such family is a set of compact generators of the unbounded derived category of quasicoherent sheaves $\mathrm{D}(Z$-qcoh) on $Z$ [Neeman 1996]; in particular, it is generated by the restrictions to $Z$ of vector bundles from $X$ (see also Lemma 2.8).

The functor $\mathbb{L} i^{*}: \mathrm{D}^{\mathrm{b}}(X$-coh $) \rightarrow \mathrm{D}^{\mathrm{b}}(Z$-coh $)$ induces a triangulated functor

$$
i^{\circ}: \mathrm{D}_{\text {Sing }}^{\mathrm{b}}(X) \longrightarrow \mathrm{D}_{\text {Sing }}^{\mathrm{b}}(Z) .
$$

Furthermore, since the sheaf $i_{*} \mathcal{O}_{Z}$ belongs to $\operatorname{Perf}(X)$, the functor $i_{*}: \mathrm{D}^{\mathrm{b}}(Z$-coh $) \rightarrow$ $\mathrm{D}^{\mathrm{b}}(X$-coh) takes $\operatorname{Perf}(Z)$ to $\operatorname{Perf}(X)$ (cf. [Orlov 2004, paragraphs before Proposition 1.14]). Hence the functor $i_{*}$ induces a triangulated functor $i_{\circ}: \mathrm{D}_{\text {Sing }}^{\mathrm{b}}(Z) \rightarrow$ $\mathrm{D}_{\text {Sing }}^{\mathrm{b}}(X)$ right adjoint to $i^{\circ}$. The triangulated category $\mathrm{D}_{\text {Sing }}^{\mathrm{b}}(Z / X)$ is the quotient category of $D_{\text {Sing }}^{b}(Z)$ by the thick subcategory generated by the image of the functor $i^{\circ}$.

When $X$ is regular, any coherent sheaf on $X$ has a finite resolution by locally free sheaves of finite rank. So $D_{\text {Sing }}^{\mathrm{b}}(X)=0$, and hence the triangulated categories $\mathrm{D}_{\text {Sing }}^{\mathrm{b}}(Z)$ and $\mathrm{D}_{\text {Sing }}^{\mathrm{b}}(Z / X)$ coincide. The converse is also true: the structure sheaf of the reduced scheme structure on the closure of any singular point of $X$ is not a perfect complex on $X$, so $\mathrm{D}_{\text {Sing }}^{\mathrm{b}}(X) \neq 0$ when $X$ is not regular.

Remark 2.1. Roughly speaking, the triangulated category of relative singularities $\mathrm{D}_{\text {Sing }}^{\mathrm{b}}(Z / X)$ measures how much worse the singularities of $Z$ are compared to the singularities of $X$ in a neighborhood of $Z$.

The basic formal properties of $D_{\text {Sing }}^{b}(Z / X)$ are similar to those of $D_{\text {Sing }}^{b}(Z)$. When the $\mathcal{O}_{X}$-module $\mathcal{O}_{Z}$ has finite flat dimension, the derived category $\mathrm{D}^{\mathrm{b}}(X$-coh) is generated by coherent sheaves adjusted to $i^{*}$. Let $\mathrm{E}_{Z / X}$ denote the minimal full subcategory of the abelian category of coherent sheaves on $Z$ containing the restrictions of such coherent sheaves from $X$ and closed under extensions and the kernels of epimorphisms of sheaves. Then $\mathrm{E}_{Z / X}$ is naturally an exact category and its bounded derived category $\mathrm{D}^{\mathrm{b}}\left(\mathrm{E}_{Z / X}\right)$ is equivalent to the thick subcategory of 
$\mathrm{D}^{\mathrm{b}}(Z$-coh) generated by the derived restrictions of coherent sheaves from $X$, so $\mathrm{D}_{\text {Sing }}^{\mathrm{b}}(Z / X)=\mathrm{D}^{\mathrm{b}}(Z$-coh $) / \mathrm{D}^{\mathrm{b}}\left(\mathrm{E}_{Z / X}\right)$. One can define the E-homological dimension of a coherent sheaf (or bounded complex) on $Z$ as the minimal length of a left resolution consisting of objects from $\mathrm{E}_{Z / X}$. This dimension does not depend on the choice of a resolution (in the same sense that the conventional flat dimension doesn't). The thick subcategory $\mathrm{D}^{\mathrm{b}}\left(\mathrm{E}_{Z / X}\right)$ consists of those objects of $\mathrm{D}^{\mathrm{b}}(Z$-coh $)$ that have finite E-homological dimensions.

Unlike in the case of perfect complexes, we do not know whether the property to belong to $\mathrm{E}_{Z / X}$ or $\mathrm{D}^{\mathrm{b}}\left(\mathrm{E}_{Z / X}\right)$ is local, though. In the case when $Z$ is a Cartier divisor, locality can be established using Theorem 2.7 below and Remark 1.3.

2.2. Matrix factorizations. Following [Polishchuk and Vaintrob 2011], we will consider matrix factorizations of a global section of a line bundle. So let $\mathcal{L}$ be a line bundle (invertible sheaf) on $X$ and $w \in \mathcal{L}(X)$ be a fixed section, called the potential.

Let $\mathcal{B}=(X, \mathcal{L}, w)$ denote the following $\mathbb{Z}$-graded quasicoherent CDG-algebra over $X$. The component $\mathcal{B}^{n}$ is isomorphic to $\mathcal{L}^{\otimes n / 2}$ for $n \in 2 \mathbb{Z}$ and vanishes for $n \in 2 \mathbb{Z}+1$, the multiplication in $\mathcal{B}$ being given by the natural isomorphisms $\mathcal{L}^{\otimes n / 2} \otimes \mathcal{O}_{X} \mathcal{L}^{\otimes m / 2} \rightarrow \mathcal{L}^{\otimes(n+m) / 2}$. For any affine open subscheme $U \subset X$, the differential on $\mathcal{B}(U)$ is zero, and the curvature element is $\left.w\right|_{U} \in \mathcal{B}^{2}(U)=\mathcal{L}(U)$. The elements $a_{U V}$ defining the restriction morphisms of CDG-rings $\mathcal{B}(V) \rightarrow \mathcal{B}(U)$ all vanish.

The category of quasicoherent $\mathbb{Z}$-graded $\mathcal{B}$-modules is equivalent to the category of quasicoherent $\mathbb{Z} / 2$-graded $\mathcal{O}_{X}$-modules, the equivalence assigning to a graded $\mathcal{B}$-module $\mathcal{M}$ the pair of $\mathcal{O}_{X}$-modules which we denote symbolically by $\mathcal{U}^{0}=\mathcal{M}^{0}$ and $\mathcal{U}^{1} \otimes \mathcal{L}^{\otimes 1 / 2}=\mathcal{M}^{1}$. Conversely, $\mathcal{M}^{n} \simeq \mathcal{U}^{n \bmod 2} \otimes_{\mathcal{O}_{X}} \mathcal{L}^{\otimes n / 2}$ for all $n \in \mathbb{Z}$ (the meaning of the notation in the right-hand side being the obvious one). This equivalence of abelian categories preserves all the properties of coherence, flatness, flat dimension, local projectivity/local freeness, etc. that we were interested in in Section 1.

Following [Lin and Pomerleano 2013], we will consider CDG-modules over $\mathcal{B}=(X, \mathcal{L}, w)$ whose underlying graded $\mathcal{B}$-modules correspond to coherent or quasicoherent $\mathcal{O}_{X}$-modules, rather than just locally free sheaves (as in the conventional matrix factorizations). A quasicoherent CDG-module over $(X, \mathcal{L}, w)$ is the same thing as a pair of quasicoherent $\mathcal{O}_{X}$-modules $\mathcal{U}^{0}$ and $\mathcal{U}^{1} \otimes \mathcal{L}^{\otimes 1 / 2}$ endowed with $\mathcal{O}_{X}$-linear morphisms $\mathcal{U}^{0} \rightarrow \mathcal{U}^{1} \otimes \mathcal{L}^{\otimes 1 / 2}$ and $\mathcal{U}^{1} \otimes \mathcal{L}^{\otimes 1 / 2} \rightarrow \mathcal{U}^{0} \otimes \mathcal{O}_{X} \mathcal{L}$ such that both compositions

$\mathcal{U}^{0} \rightarrow \mathcal{U}^{1} \otimes \mathcal{L}^{\otimes 1 / 2} \rightarrow \mathcal{U}^{0} \otimes \mathcal{O}_{X} \mathcal{L} \quad$ and $\quad \mathcal{U}^{1} \otimes \mathcal{L}^{\otimes 1 / 2} \rightarrow \mathcal{U}^{0} \otimes_{\mathcal{O}_{X}} \mathcal{L} \rightarrow \mathcal{U}^{1} \otimes_{\mathcal{O}_{X}} \mathcal{L}^{\otimes 3 / 2}$

are equal to the multiplications with $w$. 
2.3. Exotic derived categories of matrix factorizations. The following corollary is a restatement of the results of Section 1 in the application to the quasicoherent CDG-algebra $\mathcal{B}=(X, \mathcal{L}, w)$. We will use the notation $(X, \mathcal{L}, w)$-coh If $_{\text {(instead of }}$ the previously introduced $\mathcal{B}$-coh $\mathrm{fl}_{\mathrm{fl}}$ ) for the DG-category of locally free matrix factorizations of finite rank, and the notation $(X, \mathcal{L}, w)$-qcoh If (instead of the previously introduced $\mathcal{B}$-qcoh ${ }_{\text {Ip }}$ ) for the DG-category of locally free matrix factorizations of possibly infinite rank (see Remark 1.4). The rest of our notation system for various classes of quasicoherent $\mathrm{CDG}$-modules over $\mathcal{B}=(X, \mathcal{L}, w)$ remains in use.

In addition, we also denote by $(X, \mathcal{L}, w)$-qcoh ${ }_{\text {Ifd }}$ the DG-category of quasicoherent CDG-modules of finite locally free/locally projective dimension over $(X, \mathcal{L}, w)$ (see Remark 1.4 again). Let $\mathrm{D}^{\mathrm{co}}\left((X, \mathcal{L}, w)\right.$-qcoh $\left.{ }_{\text {Ifd }}\right)$ and $\mathrm{D}^{\text {abs }}\left((X, \mathcal{L}, w)\right.$-qcoh $\left.{ }_{\text {Ifd }}\right)$ be the corresponding derived categories of the second kind.

Corollary 2.3. (a) The functor $\mathrm{D}^{\mathrm{co}}\left((X, \mathcal{L}, w)\right.$-qcoh $\left.\mathrm{fl}_{\mathrm{fl}}\right) \rightarrow \mathrm{D}^{\mathrm{co}}\left((X, \mathcal{L}, w)\right.$-qcoh ffd $\left._{\text {f }}\right)$ induced by the embedding of DG-categories $(X, \mathcal{L}, w)$-qcoh $_{\mathrm{fl}} \rightarrow(X, \mathcal{L}, w)$-qcoh $_{\mathrm{ffd}}$ is an equivalence of triangulated categories.

(b) The functor $\mathrm{D}^{\mathrm{abs}}\left((X, \mathcal{L}, w)-\mathrm{qcoh}_{\mathrm{fl}}\right) \rightarrow \mathrm{D}^{\mathrm{abs}}\left((X, \mathcal{L}, w)\right.$-qcoh $\left._{\mathrm{ffd}}\right)$ induced by the embedding of DG-categories $(X, \mathcal{L}, w)$-qcoh $_{\mathrm{fl}} \rightarrow(X, \mathcal{L}, w)$-qcoh ${ }_{\mathrm{ffd}}$ is an equivalence of triangulated categories.

(c) The functors

$$
\begin{gathered}
\mathrm{D}^{\mathrm{co}}\left((X, \mathcal{L}, w)-\text { qcoh }_{\text {If }}\right) \longrightarrow \mathrm{D}^{\mathrm{co}}\left((X, \mathcal{L}, w)-\text { qcoh }_{\mathrm{Ifd}}\right), \\
\mathrm{D}^{\mathrm{abs}}\left((X, \mathcal{L}, w)-\mathrm{qcoh}_{\mathrm{If}}\right) \longrightarrow \mathrm{D}^{\mathrm{abs}}\left((X, \mathcal{L}, w)-\text { qcoh }_{\mathrm{Ifd}}\right)
\end{gathered}
$$

induced by the embedding of DG-categories $(X, \mathcal{L}, w)$-qcoh ${ }_{\mathrm{lf}} \rightarrow(X, \mathcal{L}, w)$-qcoh $\mathrm{Ifd}_{\mathrm{fd}}$ are equivalences of triangulated categories.

(d) The triangulated categories $\mathrm{D}^{\mathrm{co}}\left((X, \mathcal{L}, w)-\mathrm{qcoh}_{\mathrm{If}}\right)$ and $\mathrm{D}^{\mathrm{abs}}\left((X, \mathcal{L}, w)-\mathrm{qcoh}_{\mathrm{If}}\right)$ coincide, as do the categories $\mathrm{D}^{\mathrm{co}}\left((X, \mathcal{L}, w)\right.$-qcoh $\left.{ }_{\mathrm{lfd}}\right)$ and $\mathrm{D}^{\mathrm{abs}}\left((X, \mathcal{L}, w)-\mathrm{qcoh}_{\mathrm{lfd}}\right)$. The natural functors between these four categories form a commutative square of equivalences of triangulated categories.

(e) When the scheme $X$ has finite Krull dimension, the functors

$$
\begin{aligned}
& \mathrm{D}^{\mathrm{co}}\left((X, \mathcal{L}, w)-\mathrm{qcoh}_{\mathrm{If}}\right) \longrightarrow \mathrm{D}^{\mathrm{co}}\left((X, \mathcal{L}, w)-\mathrm{qcoh}_{\mathrm{fl}}\right), \\
& \mathrm{D}^{\mathrm{abs}}\left((X, \mathcal{L}, w)-\mathrm{qcoh}_{\mathrm{lf}}\right) \longrightarrow \mathrm{D}^{\mathrm{abs}}\left((X, \mathcal{L}, w)-\mathrm{qcoh}_{\mathrm{fl}}\right)
\end{aligned}
$$

induced by the embedding of DG-categories $(X, \mathcal{L}, w)-\mathrm{qcoh}_{\mathrm{lf}} \rightarrow(X, \mathcal{L}, w)-\mathrm{qcoh}_{\mathrm{fl}}$ are equivalences of triangulated categories. The natural functors between these four categories form a commutative square of equivalences.

(f) When the scheme $X$ has finite Krull dimension, the triangulated category $\mathrm{D}^{\mathrm{co}}\left((X, \mathcal{L}, w)\right.$-qcoh $\left.{ }_{\mathrm{fl}}\right)$ coincides with $\mathrm{D}^{\mathrm{abs}}\left((X, \mathcal{L}, w)\right.$-qcoh $\left.\mathrm{fl}_{\mathrm{fl}}\right)$ and the triangulated 
category $\mathrm{D}^{\mathrm{co}}\left((X, \mathcal{L}, w)\right.$-qcoh $\left.{ }_{\mathrm{ffd}}\right)$ coincides with $\mathrm{D}^{\mathrm{abs}}\left((X, \mathcal{L}, w)\right.$-qcoh $\left.{ }_{\mathrm{ffd}}\right)$. The natural functors between these four categories form a commutative square of equivalences.

(g) The functor $\mathrm{D}^{\mathrm{abs}}\left((X, \mathcal{L}, w)\right.$-coh $\left.\mathrm{ff}_{\mathrm{ff}}\right) \rightarrow \mathrm{D}^{\mathrm{abs}}\left((X, \mathcal{L}, w)\right.$-coh $\left.\mathrm{cffd}_{\mathrm{fd}}\right)$ induced by the embedding of $D G$-categories $(X, \mathcal{L}, w)$-coh ${ }_{\mathrm{If}} \rightarrow(X, \mathcal{L}, w)$-coh ${ }_{\mathrm{ffd}}$ is an equivalence of triangulated categories.

(h) The triangulated functors

$$
\mathrm{D}^{\text {abs }}\left((X, \mathcal{L}, w)-\text { qcoh }_{\text {If }}\right) \rightarrow \mathrm{D}^{\text {abs }}\left((X, \mathcal{L}, w) \text {-qcoh }_{\text {fl }}\right) \rightarrow \mathrm{D}^{\text {abs }}((X, \mathcal{L}, w) \text {-qcoh })
$$

induced by the embeddings of DG-categories $(X, \mathcal{L}, w)$-qcoh $\mathrm{If}_{\mathrm{f}} \rightarrow(X, \mathcal{L}, w)-\mathrm{qcoh}_{\mathrm{fl}} \rightarrow$ $(X, \mathcal{L}, w)$-qcoh are fully faithful.

(i) The triangulated functor $\mathrm{D}^{\mathrm{abs}}\left((X, \mathcal{L}, w)-\right.$ coh $\left._{\mathrm{If}}\right) \rightarrow \mathrm{D}^{\mathrm{abs}}((X, \mathcal{L}, w)$-coh) induced by the embedding of DG-categories $(X, \mathcal{L}, w)$-coh $\mathrm{I}_{\mathrm{If}} \rightarrow(X, \mathcal{L}, w)$-coh is fully faithful.

(j) The triangulated functor $\mathrm{D}^{\mathrm{abs}}\left((X, \mathcal{L}, w)\right.$-coh $\left.\mathrm{If}_{\mathrm{f}}\right) \rightarrow \mathrm{D}^{\mathrm{co}}\left((X, \mathcal{L}, w)\right.$-qcoh $\left.\cos _{\mathrm{If}}\right)$ induced by the embedding of DG-categories $(X, \mathcal{L}, w)$-coh ${ }_{\mathrm{If}} \rightarrow(X, \mathcal{L}, w)$-qcoh ${ }_{\mathrm{If}}$ is fully faithful.

(k) The triangulated functor $\mathrm{D}^{\mathrm{abs}}((X, \mathcal{L}, w)$-coh $) \rightarrow \mathrm{D}^{\mathrm{abs}}((X, \mathcal{L}, w)$-qcoh) induced by the embedding of $D G$-categories $(X, \mathcal{L}, w)$-coh $\rightarrow(X, \mathcal{L}, w)$-qcoh is fully faithful. (l) The triangulated functor $\mathrm{D}^{\mathrm{abs}}((X, \mathcal{L}, w)$-coh $) \rightarrow \mathrm{D}^{\mathrm{co}}((X, \mathcal{L}, w)$-qcoh) induced by the embedding of $D G$-categories $(X, \mathcal{L}, w)$-coh $\rightarrow(X, \mathcal{L}, w)$-qcoh is fully faithful and its image forms a set of compact generators for $\mathrm{D}^{\mathrm{co}}((X, \mathcal{L}, w)$-qcoh).

Proof. Parts (a), (b) and (g) are particular cases of Theorem 1.4, and the proof of part (c) is similar (see Remark 1.4). Part (g) also essentially follows from Proposition 1.5(b) (and part (b) can be proven similarly). Parts (h), (i), (k) and (l) are particular cases of Proposition 1.5 (except for "locally free half" of part (h), which is similar to the "flat half"). Part (d) is Theorem 1.6 together with part (c). Part (j) is Corollary 1.6. Part (e) follows from parts (a)-(c) and Remark 1.4 (cf. the discussion in the end of Section 1.6). Part (f) follows from parts (a), (b), (d) and (e); alternatively, it can be proven directly in the way similar to part (d), using the fact that the exact category of flat quasicoherent sheaves on $X$ has finite homological dimension when the Krull dimension of $X$ is finite.

2.4. Regular and Gorenstein scheme cases. When the scheme $X$ is regular or Gorenstein, the assertions of Corollary 2.3 simplify as follows.

Corollary 2.4. (a) When the scheme $X$ is Gorenstein of finite Krull dimension, the functors

$$
\mathrm{D}^{\text {abs }}\left((X, \mathcal{L}, w)-\text { qcoh }_{\mathrm{fl}}\right) \longrightarrow \mathrm{D}^{\mathrm{co}}\left((X, \mathcal{L}, w)-\mathrm{qcoh}_{\mathrm{fl}}\right) \longrightarrow \mathrm{D}^{\mathrm{co}}((X, \mathcal{L}, w)-\mathrm{qcoh})
$$


induced by the embedding of DG-categories $(X, \mathcal{L}, w)-\mathrm{qcoh}_{\mathrm{fl}} \rightarrow(X, \mathcal{L}, w)$-qcoh are equivalences of triangulated categories.

(b) When the scheme $X$ is regular of finite Krull dimension, the natural functors between the categories $\mathrm{D}^{\mathrm{abs}}\left((X, \mathcal{L}, w)-\mathrm{qcoh}_{\mathrm{fl}}\right), \mathrm{D}^{\mathrm{co}}\left((X, \mathcal{L}, w)-\mathrm{qcoh}_{\mathrm{fl}}\right)$, $\mathrm{D}^{\text {abs }}((X, \mathcal{L}, w)$-qcoh $)$, and $\mathrm{D}^{\mathrm{co}}((X, \mathcal{L}, w)$-qcoh $)$ form a commutative square of equivalences of triangulated categories.

(c) When the scheme $X$ is regular, the natural functor $\mathrm{D}^{\mathrm{abs}}\left((X, \mathcal{L}, w)-\right.$ coh $\left._{\mathrm{If}}\right) \rightarrow$ $\mathrm{D}^{\mathrm{abs}}((X, \mathcal{L}, w)$-coh) is an equivalence of triangulated categories.

Proof. Part (a) is a particular case of Proposition 1.7. Part (c) follows from Corollary $2.3(\mathrm{~g})$ since any coherent sheaf on a regular scheme has finite flat dimension. In the assumptions of part (b), the functor $\mathrm{D}^{\text {abs }}((X, \mathcal{L}, w)$-qcoh) $\rightarrow$ $\mathrm{D}^{\mathrm{co}}((X, \mathcal{L}, w)$-qcoh $)$ is an isomorphism of triangulated categories by [Positselski 2011b, Theorem 3.6(a) and Remark 3.6] since the abelian category of quasicoherent sheaves on a regular scheme of finite Krull dimension has finite homological dimension and enough injectives (cf. Theorem 1.6). The remaining assertions of part (b) follow from Corollary 2.3(a) and (b), or alternatively from part (a).

Assuming that $X$ has finite Krull dimension, the assertions of Corollaries 2.3 and 2.4 may be summarized by the following commutative diagram of triangulated functors. Here, as above, $\mathcal{B}$ denotes the quasicoherent CDG-algebra $(X, \mathcal{L}, w)$ :

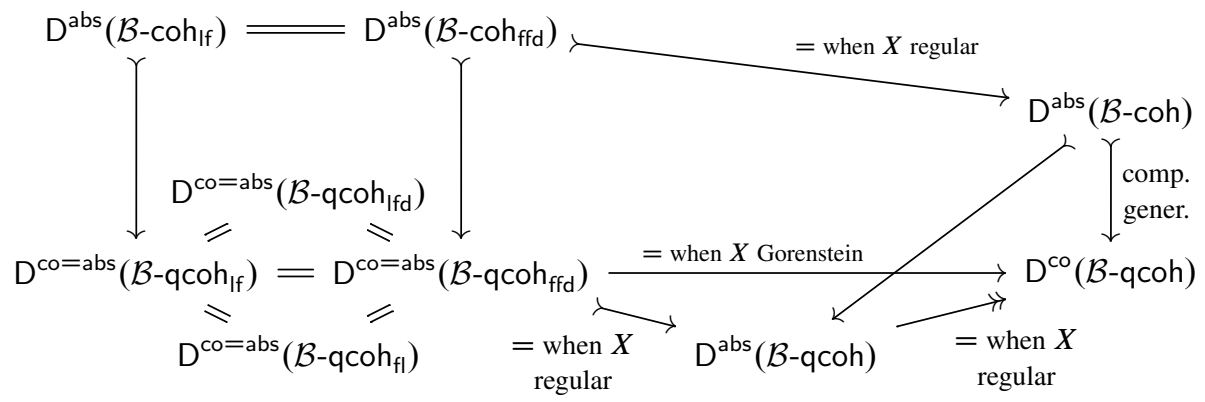

The four categories in the left lower area are coderived categories coinciding with absolute derived categories (of the same classes of quasicoherent CDG-modules). The five double lines between these four categories are equivalences, as is the upper left horizontal line. All the arrows going down are fully faithful functors. The image of the rightmost vertical arrow is a set of compact generators in the target category. The only arrow going up is a Verdier localization functor.

Nothing is claimed about the long horizontal arrow in the right lower area of the diagram in general; but when $X$ is Gorenstein, this functor is an equivalence of categories. When $X$ is regular, all the arrows going right are equivalences of categories (so the whole diagram reduces to one triangulated category with infinite direct sums, containing a full triangulated subcategory of compact generators). 
Recall also that, by Lemma 1.7, for any $X$ we have a commutative diagram of triangulated functors

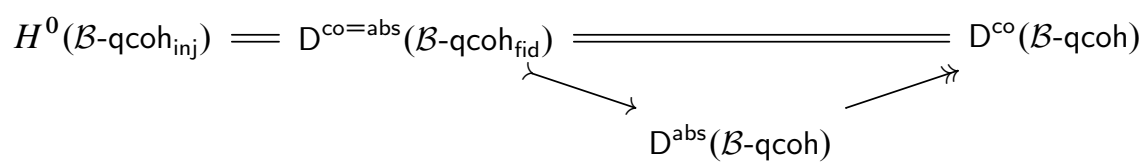

with equivalences of categories in the upper line. The fully faithful embedding $\mathrm{D}^{\mathrm{abs}}\left(\mathcal{B}\right.$-qcoh $\left.\mathrm{cod}_{\text {fid }}\right) \rightarrow \mathrm{D}^{\mathrm{abs}}(\mathcal{B}$-qcoh), which in the Gorenstein case (of finite Krull dimension) coincides with the embedding $\mathrm{D}^{\mathrm{abs}}\left(\mathcal{B}\right.$-qcoh $\left.{ }_{\mathrm{ffd}}\right) \rightarrow \mathrm{D}^{\text {abs }}(\mathcal{B}$-qcoh $)$, is always right adjoint to the localization functor $\mathrm{D}^{\text {abs }}(\mathcal{B}$-qcoh $) \rightarrow \mathrm{D}^{\mathrm{co}}(\mathcal{B}$-qcoh $)$.

Remark 2.4. When $X$ is an affine Noetherian scheme of finite Krull dimension, the embeddings of DG-categories $(X, \mathcal{L}, w)$-qcoh $\cos _{\mathrm{p}} \rightarrow(X, \mathcal{L}, w)$-qcoh $_{\mathrm{fl}} \rightarrow$ $(X, \mathcal{L}, w)$-qcoh induce equivalences $H^{0}\left(\mathcal{B}\right.$-qcoh Ip $\left._{\mathrm{p}}\right) \simeq \mathrm{D}^{\text {abs }}\left(\mathcal{B}\right.$-qcoh $\left.{ }_{\mathrm{fl}}\right) \simeq \mathrm{D}^{\mathrm{ctr}}(\mathcal{B}$-qcoh $)$ between the homotopy category of (locally) projective matrix factorizations of infinite rank, the absolute derived category of flat matrix factorizations, and the contraderived category of arbitrary quasicoherent matrix factorizations (see [Positselski 2011b, Section 3.8]; cf. Remark 1.5).

2.5. Serre-Grothendieck duality. The aim of this section is to show that the somewhat mysterious long horizontal arrow in the above large diagram is actually a functor between two equivalent triangulated categories, for a rather wide class of schemes $X$. The functor $\mathrm{D}^{\mathrm{co}}\left((X, \mathcal{L}, w)\right.$-qcoh $\left._{\mathrm{fl}}\right) \rightarrow \mathrm{D}^{\mathrm{co}}((X, \mathcal{L}, w)$-qcoh) in the above diagram, which is induced by the embedding of DG-categories $(X, \mathcal{L}, w)$-qcoh $_{\mathrm{fl}} \rightarrow(X, \mathcal{L}, w)$-qcoh, is not the equivalence that we have in mind, however (unless the scheme is Gorenstein). Instead, the equivalence between the categories $\mathrm{D}^{\mathrm{co}}\left((X, \mathcal{L}, w)\right.$-qcoh $\left.{ }_{\mathrm{fl}}\right)$ and $\mathrm{D}^{\mathrm{co}}((X, \mathcal{L}, w)$-qcoh) is constructed using a dualizing complex on $X$ [Hartshorne 1966, Section V.2].

Before recalling the definition of a dualizing complex, let us discuss the notion of the quasicoherent internal Hom. Given quasicoherent sheaves $\mathcal{M}$ and $\mathcal{N}$ over $X$, the quasicoherent sheaf $\mathcal{H o m}_{X \text {-qc }}(\mathcal{M}, \mathcal{N})$ is defined by the isomorphism $\operatorname{Hom}_{\mathcal{O}_{X}}\left(-\otimes_{\mathcal{O}_{X}} \mathcal{M}, \mathcal{N}\right) \simeq \operatorname{Hom}_{\mathcal{O}_{X}}\left(-, \mathcal{H} \operatorname{mom}_{X \text {-qc }}(\mathcal{M}, \mathcal{N})\right)$ of functors from the category of quasicoherent sheaves to the category of abelian groups. Equivalently, the quasicoherent sheaf $\mathcal{H o m}_{X \text {-qc }}(\mathcal{M}, \mathcal{N})$ can be obtained by applying the coherator functor [Thomason and Trobaugh 1990, Sections B.12-B.14] to the sheaf of $\mathcal{O}_{X}$-modules $\operatorname{Hom}_{\mathcal{O}_{X}}(\mathcal{M}, \mathcal{N})$. Whenever $\mathcal{M}$ is a coherent sheaf, the sheaf $\operatorname{Hom}_{\mathcal{O}_{X}}(\mathcal{M}, \mathcal{N})$ of $\mathcal{O}_{X}$-module internal Hom is quasicoherent, and $\mathcal{H o m}_{X \text {-qc }}(\mathcal{M}, \mathcal{N}) \simeq \mathcal{H o m}_{\mathcal{O}_{X}}(\mathcal{M}, \mathcal{N})$.

Notice that the construction of the sheaf $\mathcal{H o m}_{X \text {-qc }}(\mathcal{M}, \mathcal{N})$ is not local in general; i.e., it does not commute with the restrictions of quasicoherent sheaves to open subschemes; when the sheaf $\mathcal{M}$ is coherent, it does. 
Lemma 2.5. (a) For any injective quasicoherent sheaf $\mathcal{J}$ over a separated Noetherian scheme $X$, the functor $\mathcal{M} \longmapsto \mathcal{H o m}_{X \text {-qc }}(\mathcal{M}, \mathcal{J})$ is exact.

(b) For any flat quasicoherent sheaf $\mathcal{F}$ and injective quasicoherent sheaf $\mathcal{J}$ over $X$, the quasicoherent sheaves $\mathcal{F} \otimes_{\mathcal{O}_{X}} \mathcal{J}$ and $\mathcal{H o m}_{X \text {-qc }}(\mathcal{F}, \mathcal{J})$ are injective.

(c) For any injective quasicoherent sheaves $\mathcal{J}^{\prime}$ and $\mathcal{J}$ over $X$, the quasicoherent sheaf $\mathcal{H o m}_{X \text {-qc }}\left(\mathcal{J}^{\prime}, \mathcal{J}\right)$ is flat.

Proof. The second assertion of part (b) is obvious from the universal property defining $\mathcal{H o m}_{X \text {-qc }}$. To prove the first one, notice that injectivity of quasicoherent sheaves over a Noetherian scheme is a local property ([Hartshorne 1966, Lemma II.7.16 and Theorem II.7.18] or Theorem A.3), a flat quasicoherent sheaf over an affine scheme is a filtered inductive limit of locally free sheaves of finite rank [Bourbaki 1980, Numéros 1.5-6], and injectivity of modules over a Noetherian ring is preserved by filtered inductive limits.

The proof of parts (a) and (c) follows the argument in [Murfet 2007, Lemma 8.7]. Choose a finite affine covering $U_{\alpha}$ of the scheme $X$ and consider the morphism $\mathcal{J} \rightarrow \bigoplus_{\alpha} j_{U_{\alpha} *} j_{U_{\alpha}}^{*} \mathcal{J}$. Being an embedding of injective quasicoherent sheaves, it splits, so $\mathcal{J}$ is a direct summand of the direct sum of $j_{U_{\alpha} *} j_{U_{\alpha}}^{*} \mathcal{J}$. Hence it suffices to prove both assertions in the case when $\mathcal{J}=j_{V *} \mathcal{J}^{\prime \prime}$, where $\mathcal{J}^{\prime \prime}$ is an injective quasicoherent sheaf on an affine open subscheme $V \subset X$.

Now we have $\mathcal{H o m}_{X \text {-qc }}\left(\mathcal{M}, j_{V *} \mathcal{J}^{\prime \prime}\right) \simeq j_{V *} \mathcal{H o m}_{V \text {-qc }}\left(j_{V}^{*} \mathcal{M}, \mathcal{J}^{\prime \prime}\right)$. Since $V \rightarrow X$ is a flat affine morphism, the functor $j_{V *}$ is exact and preserves the flatness of quasicoherent sheaves. This proves part (a) and reduces (c) to the case of an affine scheme $X=V$. It remains to apply [Cartan and Eilenberg 1956, Proposition VI.5.3].

For our purposes, a dualizing complex $\mathcal{D}_{X}^{\bullet}$ on $X$ is a finite complex of injective quasicoherent sheaves such that the cohomology sheaves of $\mathcal{D}_{X}^{\bullet}$ are coherent and for any coherent sheaf $\mathcal{M}$ over $X$, the natural morphism of finite complexes of quasicoherent sheaves $\mathcal{M} \rightarrow \mathcal{H o m}_{X \text {-qc }}\left(\mathcal{H o m}_{X \text {-qc }}\left(\mathcal{M}, \mathcal{D}_{X}^{\bullet}\right), \mathcal{D}_{X}^{\bullet}\right)$ is a quasi-isomorphism. Note that it follows from the former two conditions on $\mathcal{D}_{X}^{\bullet}$ that the complex $\mathcal{H o m}_{X \text {-qc }}\left(\mathcal{M}, \mathcal{D}_{X}^{\bullet}\right)$ has coherent cohomology sheaves. This makes the conditions imposed on $\mathcal{D}_{X}^{\bullet}$ actually local in $X$, so the restriction $\mathcal{D}_{U}^{\bullet}=\left.\mathcal{D}_{X}^{\bullet}\right|_{U}$ of the complex of sheaves $\mathcal{D}_{X}^{\circ}$ to an open subscheme $U \subset X$ is a dualizing complex on $U$.

Given a quasicoherent CDG-algebra $\mathcal{B}$ over $X$, a quasicoherent left CDG-module $\mathcal{M}$ over $\mathcal{B}$, and a complex of quasicoherent sheaves $\mathcal{F}^{\bullet}$ on $X$, consider the complexes of quasicoherent left CDG-modules $\mathcal{F}^{\bullet} \otimes_{\mathcal{O}_{X}} \mathcal{M}$ and $\mathcal{H o m}_{X \text {-qc }}\left(\mathcal{F}^{\bullet}, \mathcal{M}\right)$ over $\mathcal{B}$. Taking their totalizations (formed, if necessary, by taking infinite direct sums along the diagonals), construct two triangulated functors $H^{0}(\mathcal{B}$-qcoh $) \rightarrow H^{0}(\mathcal{B}$-qcoh) depending on a complex $\mathcal{F}^{\bullet}$. Given a right CDG-module $\mathcal{N}$ over $\mathcal{B}$ (see [Positselski 2011b, Sections 3.1 and B.1]), similarly construct a complex of quasicoherent left 
CDG-modules $\operatorname{Hom}_{X \text {-qc }}\left(\mathcal{N}, \mathcal{F}^{\bullet}\right)$ over $\mathcal{B}$, obtaining a triangulated functor from the homotopy category of right CDG-modules $H^{0}$ (qcoh- $\left.\mathcal{B}\right)$ to $H^{0}(\mathcal{B}$-qcoh).

In the particular case of matrix factorizations, we conclude that the covariant functors $\mathcal{F}^{\bullet} \otimes_{\mathcal{O}_{X}}-$ and $\mathcal{H o m}_{X \text {-qc }}\left(\mathcal{F}^{\bullet},-\right)$ take quasicoherent matrix factorizations of a potential $w \in \mathcal{L}(X)$ to (complexes of) quasicoherent matrix factorizations of $w$, while the contravariant functor $\mathcal{H o m}_{X \text {-qc }}\left(-, \mathcal{F}^{\bullet}\right)$ transforms quasicoherent matrix factorizations of the opposite potential $-w \in \mathcal{L}(X)$ into (complexes of) quasicoherent matrix factorizations of $w$. Of course, the quasicoherent CDG-algebras $(X, \mathcal{L}, w)$ and $(X, \mathcal{L},-w)$ over a scheme $X$ are naturally isomorphic, but we prefer to keep the distinction between the two.

The next proposition provides the matrix factorization version of the conventional (contravariant) Serre-Grothendieck duality for bounded complexes of coherent sheaves. We assume that $X$ is a separated Noetherian scheme with a dualizing complex $\mathcal{D}_{X}^{\bullet}$. Recall that any such scheme has finite Krull dimension [Hartshorne 1966, Corollary V.7.2]. We denote by D ${ }^{\text {op }}$ the opposite category to a category D.

\section{Proposition 2.5. The triangulated functor}

$$
\mathcal{H o m}_{X \text {-qc }}\left(-, \mathcal{D}_{X}^{\bullet}\right): H^{0}((X, \mathcal{L},-w) \text {-qcoh })^{\mathrm{op}} \longrightarrow H^{0}((X, \mathcal{L}, w) \text {-qcoh })
$$

induces a well-defined triangulated functor between the absolute derived categories $\mathrm{D}^{\mathrm{abs}}((X, \mathcal{L},-w) \text {-qcoh })^{\mathrm{op}}$ and $\mathrm{D}^{\mathrm{abs}}((X, \mathcal{L}, w)$-qcoh) taking the full triangulated subcategory $\mathrm{D}^{\mathrm{abs}}((X, \mathcal{L},-w) \text {-coh })^{\mathrm{op}} \subset \mathrm{D}^{\mathrm{abs}}((X, \mathcal{L},-w) \text {-qcoh })^{\text {op }}$ into the full subcategory $\mathrm{D}^{\mathrm{abs}}((X, \mathcal{L}, w)$-coh $) \subset \mathrm{D}^{\mathrm{abs}}((X, \mathcal{L}, w)$-qcoh). The composition of the duality functors $\mathrm{D}^{\text {abs }}((X, \mathcal{L}, w)$-coh $) \rightarrow \mathrm{D}^{\text {abs }}((X, \mathcal{L},-w) \text {-coh })^{\text {op }} \rightarrow \mathrm{D}^{\text {abs }}((X, \mathcal{L}, w)$-coh $)$ is the identity functor.

Proof. The functor $\mathcal{H} m_{X \text {-qc }}\left(-, \mathcal{D}_{X}^{\bullet}\right)$ preserves absolute acyclicity, because $\mathcal{D}_{X}^{\bullet}$ is a complex of injective quasicoherent sheaves, so Lemma 2.5(a) applies. Given a coherent matrix factorization $\mathcal{M}$, the finite complex of matrix factorizations $\mathcal{H}_{\text {om }}{ }_{X \text {-qc }}\left(-, \mathcal{D}_{X}^{\bullet}\right)$ has coherent cohomology matrix factorizations, so one can use its canonical truncations in order to prove by induction that its totalization belongs to the triangulated subcategory $\mathrm{D}^{\text {abs }}((X, \mathcal{L}, w)$-coh).

Finally, for any quasicoherent matrix factorization $\mathcal{M}$, consider the bicomplex of matrix factorizations $\mathcal{H o m}_{X \text {-qc }}\left(\mathcal{H o m}_{X \text {-qc }}\left(\mathcal{M}, \mathcal{D}_{X}^{\bullet}\right), \mathcal{D}_{X}^{\bullet}\right)$ and take its totalization in the two directions where it is a complex, obtaining a complex of matrix factorizations. Then there is a natural morphism of finite complexes of matrix factorizations $\mathcal{M} \rightarrow$ $\mathcal{H o m}_{X \text {-qc }}\left(\mathcal{H o m}_{X \text {-qc }}\left(\mathcal{M}, \mathcal{D}_{X}^{\bullet}\right), \mathcal{D}_{X}^{\bullet}\right)$, which is a quasi-isomorphism of complexes of matrix factorizations when $\mathcal{M}$ is coherent. The induced closed morphism of the total matrix factorizations is an isomorphism in $\mathrm{D}^{\text {abs }}((X, \mathcal{L}, w)$-qcoh) since the totalization of a finite acyclic complex of matrix factorizations is absolutely acyclic. It 
remains to use the fact that the functor $\mathrm{D}^{\text {abs }}((X, \mathcal{L}, w)$-coh $) \rightarrow \mathrm{D}^{\text {abs }}((X, \mathcal{L}, w)$-qcoh $)$ is fully faithful (see Corollary $2.3(\mathrm{k}))$ again.

The next result is our covariant Serre-Grothendieck duality theorem for matrix factorizations. It is the matrix factorization analogue of the similar results for complexes of projective and injective modules [Iyengar and Krause 2006, Theorem 4.2] and sheaves [Murfet 2007, Theorem 8.4]. It also strongly resembles the derived comodule-contramodule correspondence theory (see [Positselski 2010, Corollaries 5.4 and 6.3; 2011b, Theorem 5.2]; cf. Remark 2.4 above). Notice that our proof is more akin to the arguments in [Positselski 2010; 2011b] than those of [Iyengar and Krause 2006; Murfet 2007] in that we give a direct proof of the covariant duality independent of both the contravariant duality and any descriptions of the compact objects in the categories to be compared.

Theorem 2.5. The functors

$$
\begin{gathered}
\mathcal{D}_{X}^{\bullet} \otimes_{\mathcal{O}_{X}}-: H^{0}\left((X, \mathcal{L}, w)-\text { qcoh }_{\mathrm{fl}}\right) \longrightarrow H^{0}\left((X, \mathcal{L}, w) \text {-qcoh }_{\text {inj }}\right), \\
\mathcal{H o m}_{X \text {-qc }}\left(\mathcal{D}_{X}^{\bullet},-\right): H^{0}\left((X, \mathcal{L}, w) \text {-qcoh }_{\text {inj }}\right) \longrightarrow H^{0}\left((X, \mathcal{L}, w) \text {-qcoh }_{\mathrm{fl}}\right)
\end{gathered}
$$

induce mutually inverse equivalences between the coderived categories

$$
\mathrm{D}^{\mathrm{co}}\left((X, \mathcal{L}, w)-\mathrm{qcoh}_{\mathrm{fl}}\right) \text { and } \mathrm{D}^{\mathrm{co}}((X, \mathcal{L}, w) \text {-qcoh }) .
$$

Proof. Recall that $H^{0}\left((X, \mathcal{L}, w)\right.$-qcoh $\left.{ }_{\text {inj }}\right) \simeq \mathrm{D}^{\mathrm{co}}((X, \mathcal{L}, w)$-qcoh) by Lemma 1.7(b) and $\mathrm{D}^{\text {abs }}\left((X, \mathcal{L}, w)-\mathrm{qcoh}_{\mathrm{fl}}\right)=\mathrm{D}^{\mathrm{co}}\left((X, \mathcal{L}, w)\right.$-q $\left.\operatorname{coh}_{\mathrm{fl}}\right)$ by Corollary 2.3(f) (though we will reprove the latter fact rather than use it in the following argument; see also Remark 2.6 below and Lemma A.1). The functor

$$
\mathcal{D}_{X}^{\bullet} \otimes_{\mathcal{O}_{X}}-: H^{0}\left((X, \mathcal{L}, w)-\text { qcoh }_{\mathrm{fl}}\right) \longrightarrow H^{0}\left((X, \mathcal{L}, w)-\text { qcoh }_{\text {inj }}\right)
$$

obviously takes matrix factorizations coacyclic with respect to $(X, \mathcal{L}, w)$-qcoh $\mathrm{fl}_{\mathrm{fl}}$ to matrix factorizations coacyclic with respect to $(X, \mathcal{L}, w)$-qcoh ${ }_{\mathrm{inj}}$, which are all contractible. It remains to check that the induced functors are mutually inverse.

Let $\mathcal{E}$ be a matrix factorization from $(X, \mathcal{L}, w)$-qcoh $_{\mathrm{fl}}$. As in the previous proof, consider the bicomplex of matrix factorizations $\mathcal{H o m}_{X \text {-qc }}\left(\mathcal{D}_{X}^{\bullet}, \mathcal{D}_{X}^{\bullet} \otimes_{\mathcal{O}_{X}} \mathcal{E}\right)$ and take its total complex of matrix factorizations. Then there is a natural morphism $\mathcal{E} \rightarrow \mathcal{H o m}_{X \text {-qc }}\left(\mathcal{D}_{X}^{\bullet}, \mathcal{D}_{X}^{\bullet} \otimes_{\mathcal{O}_{X}} \mathcal{E}\right)$ of finite complexes of matrix factorizations from $(X, \mathcal{L}, w)$-qcoh $\mathrm{fl}_{\mathrm{fl}}$. To prove that the induced morphism of the total matrix factorizations is an isomorphism in $\mathrm{D}^{\mathrm{co}}\left((X, \mathcal{L}, w)\right.$-qcoh $\left._{\mathrm{fl}}\right)$, we once again use the fact that the totalization of a finite acyclic complex of matrix factorizations is absolutely acyclic. So it suffices to check that for any flat quasicoherent sheaf $\mathcal{F}$ over $X$, the natural morphism $\mathcal{F} \rightarrow \mathcal{H} \operatorname{mom}_{X \text {-qc }}\left(\mathcal{D}_{X}^{\bullet}, \mathcal{D}_{X}^{\bullet} \otimes_{\mathcal{O}_{X}} \mathcal{F}\right)$ is a quasi-isomorphism of complexes of flat quasicoherent sheaves. This will be done below. 
Similarly, let $\mathcal{M}$ be a matrix factorization from $(X, \mathcal{L}, w)$-qcoh ${ }_{\text {inj }}$. Consider the morphism of finite complexes of injective matrix factorizations given by $\mathcal{D}_{X}^{\bullet} \otimes_{\mathcal{O}_{X}} \mathcal{H} o_{X \text {-qc }}\left(\mathcal{D}_{X}^{\bullet}, \mathcal{M}\right) \rightarrow \mathcal{M}$. To prove that the cone of the induced morphism of the total matrix factorizations is contractible, it suffices to check that for any injective quasicoherent sheaf $\mathcal{J}$ over $X$, the natural morphism of complexes of injective sheaves $\mathcal{D}_{X}^{\bullet} \otimes_{\mathcal{O}_{X}} \mathcal{H o m}_{X \text {-qc }}\left(\mathcal{D}_{X}^{\bullet}, \mathcal{J}\right) \rightarrow \mathcal{J}$ is a quasi-isomorphism.

Let ' $\mathcal{D}_{X}^{\circ}$ denote a finite complex of coherent sheaves over $X$ endowed with a quasi-isomorphism ' $\mathcal{D}_{X}^{\bullet} \rightarrow \mathcal{D}_{X}^{\bullet}$. Then the morphism $\mathcal{H o m}_{X \text {-qc }}\left(\mathcal{D}_{X}^{\bullet}, \mathcal{D}_{X}^{\bullet} \otimes_{\mathcal{O}_{X}} \mathcal{F}\right) \rightarrow$ $\mathcal{H} m_{X \text {-qc }}\left({ }^{\prime} \mathcal{D}_{X}^{\bullet}, \mathcal{D}_{X}^{\bullet} \otimes_{\mathcal{O}_{X}} \mathcal{F}\right)$ is a quasi-isomorphism for any flat quasicoherent sheaf $\mathcal{F}$. The construction of the composition

$$
\mathcal{F} \longrightarrow \mathcal{H o m}_{X-\mathrm{qc}}\left(\mathcal{D}_{X}^{\bullet}, \mathcal{D}_{X}^{\bullet} \otimes_{\mathcal{O}_{X}} \mathcal{F}\right) \longrightarrow \mathcal{H o m}_{X \text {-qc }}\left({ }^{\prime} \mathcal{D}_{X}^{\bullet}, \mathcal{D}_{X}^{\bullet} \otimes_{\mathcal{O}_{X}} \mathcal{F}\right)
$$

is local in $X$, so it suffices to check that the composition is a quasi-isomorphism when $X$ is affine. Then, using the passage to the filtered inductive limit, we may assume that $\mathcal{F}$ is locally free of finite rank, and further that $\mathcal{F}=\mathcal{O}_{X}$. It remains to recall that the morphism $\mathcal{O}_{X} \rightarrow \mathcal{H o m}_{X \text {-qc }}\left({ }^{\prime} \mathcal{D}_{X}^{\bullet}, \mathcal{D}_{X}^{\bullet}\right)$ is a quasi-isomorphism by the definition of $\mathcal{D}_{X}^{\bullet}$.

Let " $\mathcal{D}_{X}^{\bullet}$ be a bounded-above complex of flat quasicoherent sheaves mapping quasi-isomorphically to ${ }^{\prime} \mathcal{D}_{X}^{\bullet}$. Then for any injective quasicoherent sheaf $\mathcal{J}$ over $X$ there are quasi-isomorphisms

$$
\begin{aligned}
& { }^{\prime \prime} \mathcal{D}_{X}^{\bullet} \otimes_{\mathcal{O}_{X}} \mathcal{H o m}_{X \text {-qc }}\left(\mathcal{D}_{X}^{\bullet}, \mathcal{J}\right) \longrightarrow \mathcal{D}_{X}^{\bullet} \otimes_{\mathcal{O}_{X}} \mathcal{H o m}_{X \text {-qc }}\left(\mathcal{D}_{X}^{\bullet}, \mathcal{J}\right), \\
& { }^{\prime \prime} \mathcal{D}_{X}^{\bullet} \otimes_{\mathcal{O}_{X}} \mathcal{H o m}_{X \text {-qc }}\left(\mathcal{D}_{X}^{\bullet}, \mathcal{J}\right) \longrightarrow{ }^{\prime \prime} \mathcal{D}_{X}^{\bullet} \otimes_{\mathcal{O}_{X}} \mathcal{H o m}_{X \text {-qc }}\left({ }^{\prime} \mathcal{D}_{X}^{\bullet}, \mathcal{J}\right)
\end{aligned}
$$

forming a commutative diagram with the evaluation morphisms into $\mathcal{J}$. Hence it remains to check that the morphism " $\mathcal{D}_{X}^{\bullet} \otimes_{\mathcal{O}_{X}} \mathcal{H o m}_{X \text {-qc }}\left({ }^{\prime} \mathcal{D}_{X}^{\bullet}, \mathcal{J}\right) \rightarrow \mathcal{J}$ is a quasi-isomorphism, which is a local question. Assume further that ${ }^{\prime \prime} \mathcal{D}_{X}^{\bullet}$ is a bounded-above complex of locally free sheaves of finite rank. Then there is a natural isomorphism of complexes of sheaves

$$
{ }^{\prime \prime} \mathcal{D}_{X}^{\bullet} \otimes_{\mathcal{O}_{X}} \mathcal{H o m}_{X \text {-qc }}\left({ }^{\prime} \mathcal{D}_{X}^{\bullet}, \mathcal{J}\right) \simeq \mathcal{H o m}_{X \text {-qc }}\left(\mathcal{H o m}_{X \text {-qc }}\left(" \mathcal{D}_{X}^{\bullet},{ }^{\prime} \mathcal{D}_{X}^{\bullet}\right), \mathcal{J}\right) .
$$

The related morphism

$$
\mathcal{H o m}_{X \text {-qc }}\left(\mathcal{H o m}_{X \text {-qc }}\left({ }^{\prime \prime} \mathcal{D}_{X}^{\bullet}, \mathcal{D}_{X}^{\bullet}\right), \mathcal{J}\right) \longrightarrow \mathcal{J}
$$

is induced by the natural morphism of complexes $\mathcal{O}_{X} \rightarrow \mathcal{H o m}_{X \text {-qc }}\left({ }^{\prime \prime} \mathcal{D}_{X}^{\bullet},{ }^{\prime} \mathcal{D}_{X}^{\bullet}\right)$. The latter is again a quasi-isomorphism essentially by the definition of $\mathcal{D}_{X}^{\bullet}$.

From this point on we resume assuming that $X$ has enough vector bundles.

Notice that the equivalence functor

$$
\mathcal{D}_{X}^{\bullet} \otimes_{\mathcal{O}_{X}}-: \mathrm{D}^{\mathrm{co}}\left((X, \mathcal{L}, w)-\mathrm{q} \operatorname{coh}_{\mathrm{lf}}\right) \longrightarrow \mathrm{D}^{\mathrm{co}}((X, \mathcal{L}, w)-\mathrm{qcoh})
$$


that we constructed takes the full triangulated subcategory $\mathrm{D}^{\text {abs }}\left((X, \mathcal{L}, w)\right.$-coh $\left.{ }_{\text {If }}\right) \subset$ $\mathrm{D}^{\mathrm{co}}\left((X, \mathcal{L}, w)\right.$-qcoh $\left.{ }_{\text {If }}\right)$ into the full triangulated subcategory $\mathrm{D}^{\text {abs }}((X, \mathcal{L}, w)$-coh $) \subset$ $\mathrm{D}^{\mathrm{co}}\left((X, \mathcal{L}, w)\right.$-qcoh). This is so because the dualizing complex $\mathcal{D}_{X}^{\bullet}$ has bounded coherent cohomology sheaves.

Now we will use Proposition 2.5 and Theorem 2.5 in order to construct compact generators of the triangulated category $\mathrm{D}^{\mathrm{co}}\left((X, \mathcal{L}, w)\right.$-qcoh $\left.{ }_{\text {If }}\right)$ (cf. [Jørgensen 2005; Neeman 2008]).

Consider the abelian category $Z^{0}((X, \mathcal{L},-w)$-coh) of coherent matrix factorizations of $-w$ and closed morphisms of degree 0 between them, and its exact subcategory of locally free matrix factorizations of finite rank $Z^{0}\left((X, \mathcal{L},-w)\right.$-coh If $\left._{\text {f }}\right)$. The natural functor between the bounded-above derived categories of our abelian category and its exact subcategory

$$
\mathrm{D}^{-}\left(Z^{0}\left((X, \mathcal{L},-w)-\text { coh }_{\text {If }}\right)\right) \longrightarrow \mathrm{D}^{-}\left(Z^{0}((X, \mathcal{L},-w)-\text { coh })\right)
$$

is an equivalence of triangulated categories. The vector bundle duality functor $\mathcal{H o m}_{X \text {-qc }}\left(-, \mathcal{O}_{X}\right): Z^{0}\left((X, \mathcal{L},-w) \text {-coh }{ }_{\text {If }}\right)^{\text {op }} \rightarrow Z^{0}\left((X, \mathcal{L}, w)\right.$-coh $\left.{ }_{\text {If }}\right)$ induces a triangulated functor $\mathrm{D}^{-}\left(Z^{0}\left((X, \mathcal{L},-w) \text {-coh }{ }_{\mathrm{If}}\right)\right)^{\mathrm{op}} \rightarrow \mathrm{D}^{+}\left(Z^{0}\left((X, \mathcal{L}, w)\right.\right.$-coh $\left.\left.\mathrm{If}_{\mathrm{ff}}\right)\right)$ taking bounded-above complexes to bounded-below ones.

Let $\mathrm{D}^{+}\left(Z^{0}\left((X, \mathcal{L}, w)\right.\right.$-qcoh If $\left.\left._{1}\right)\right)$ denote the bounded-below derived category of the exact category of locally free matrix factorizations of possibly infinite rank. Since the bounded-below acyclic complexes over any exact category with infinite direct sums are coacyclic [Positselski 2010, Lemma 2.1], there is a well-defined, triangulated direct sum totalization functor $\mathrm{D}^{+}\left(Z^{0}\left((X, \mathcal{L}, w)\right.\right.$-qcoh $\left.\left.{ }_{\text {If }}\right)\right) \rightarrow \mathrm{D}^{\mathrm{co}}\left((X, \mathcal{L}, w)-\mathrm{qcoh}_{\text {If }}\right)$. Consider the composition

$$
\begin{aligned}
& Z^{0}((X, \mathcal{L},-w)-\mathrm{coh})^{\mathrm{op}} \longrightarrow \mathrm{D}^{-}\left(Z^{0}((X, \mathcal{L},-w)-\mathrm{coh})\right)^{\mathrm{op}} \\
& \simeq \mathrm{D}^{-}\left(Z^{0}\left((X, \mathcal{L},-w)-\operatorname{coh}_{\mathrm{If}}\right)\right)^{\mathrm{op}} \longrightarrow \mathrm{D}^{+}\left(Z^{0}\left((X, \mathcal{L}, w)-\operatorname{coh}_{\mathrm{If}}\right)\right) \\
& \quad \longrightarrow \mathrm{D}^{+}\left(Z^{0}\left((X, \mathcal{L}, w)-\mathrm{qcoh}_{\mathrm{If}}\right)\right) \longrightarrow \mathrm{D}^{\mathrm{co}}\left((X, \mathcal{L}, w)-\mathrm{qcoh}_{\mathrm{If}}\right),
\end{aligned}
$$

where two of the functors are the duality and the totalization discussed above, while the other two are the natural embedding and the functor induced by such.

One easily checks that this composition takes cones of closed morphisms in $Z^{0}((X, \mathcal{L},-w)$-coh $)$ to cocones in $\mathrm{D}^{\mathrm{co}}\left((X, \mathcal{L}, w)\right.$-qcoh $\left.{ }_{\text {If }}\right)$; hence it induces a triangulated functor $H^{0}((X, \mathcal{L},-w) \text {-coh })^{\mathrm{op}} \rightarrow \mathrm{D}^{\mathrm{co}}\left((X, \mathcal{L}, w)\right.$-qcoh $\left.{ }_{\text {lf }}\right)$. Similarly, the above composition takes the totalizations of short exact sequences in $(X, \mathcal{L},-w)$-coh to objects corresponding to the totalizations of short exact sequences in $(X, \mathcal{L}, w)$-qcoh if $_{\text {; }}$; one checks this by considering a left locally free resolution of a short exact sequence of coherent matrix factorizations. Thus we obtain a triangulated functor

$$
\Omega: \mathrm{D}^{\mathrm{abs}}((X, \mathcal{L},-w)-\mathrm{coh})^{\mathrm{op}} \longrightarrow \mathrm{D}^{\mathrm{co}}\left((X, \mathcal{L}, w)-\text { qcoh }_{\text {If }}\right) .
$$


Corollary 2.5. The functor $\Omega$ is fully faithful, and its image forms a set of compact generators in $\mathrm{D}^{\mathrm{co}}\left((X, \mathcal{L}, w)\right.$-qcoh $\left.{ }_{\mathrm{lf}}\right)$. The following diagram of triangulated functors is commutative:

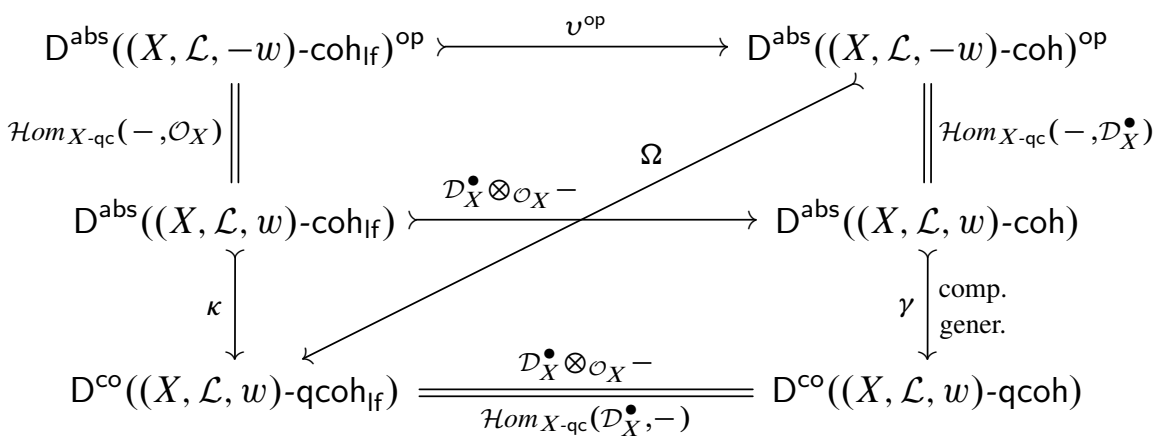

Here $v, \kappa$, and $\gamma$ denote the fully faithful functors induced by the natural embeddings of DG-categories of CDG-modules. The two upper vertical lines are the natural contravariant dualities (antiequivalences) on the (absolute derived) categories of locally free matrix factorizations of finite rank and coherent matrix factorizations. The lower horizontal line is the equivalence of categories from Theorem 2.5, and the middle horizontal arrow is the fully faithful functor discussed after the proof of Theorem 2.5.

The above diagram is to be compared with the following subdiagram of the large diagram in the end of Section 2.4:

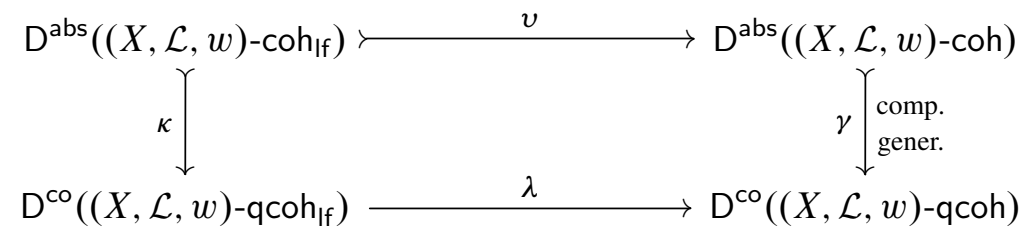

Here $\lambda$ denotes the triangulated functor induced by the embedding of DG-categories of CDG-modules $(X, \mathcal{L}, w)$-qcoh ${ }_{\text {If }} \rightarrow(X, \mathcal{L}, w)$-qcoh.

Notice that it is clear from these two diagrams that the functor $\lambda$ is an equivalence of triangulated categories whenever the functor $v$ is. Indeed, if $v$ is an equivalence of categories, then the image of $\kappa$ is a set of compact generators in the target category, and $\lambda$ is an infinite direct sum-preserving triangulated functor identifying triangulated subcategories of compact generators, and hence $\lambda$ is an equivalence. In this case, the functor $\mathcal{D}_{X}^{\bullet} \otimes_{\mathcal{O}_{X}}$ - becomes an autoequivalence of the triangulated category $\mathrm{D}^{\mathrm{co}}((X, \mathcal{L}, w)$-qcoh) and restricts to an autoequivalence of its full subcategory of compact generators $\mathrm{D}^{\text {abs }}((X, \mathcal{L}, w)$-coh).

Proof of Corollary 2.5. The assertions in the first sentence follow from the second one, as we know $\gamma$ to be fully faithful and its image to be a set of compact generators 
by Corollary 2.3(1). The commutativity of both squares and the upper left triangle is clear. To check commutativity of the lower right triangle, consider a coherent matrix factorization $\mathcal{M}$ of the potential $-w$; let $\mathcal{E}_{\bullet}$ be its left resolution in the abelian category $Z^{0}\left((X, \mathcal{L},-w)\right.$-coh) whose terms $\mathcal{E}_{n}$ belong to $Z^{0}\left((X, \mathcal{L},-w)\right.$-coh $\left.{ }_{\text {If }}\right)$. Then the finite complex of matrix factorizations $\mathcal{H o m}_{X \text {-qc }}\left(\mathcal{M}, \mathcal{D}_{X}^{\bullet}\right)$ maps quasiisomorphically to the bounded-below complex of injective matrix factorizations $\mathcal{H o m}_{X \text {-qc }}\left(\mathcal{E}_{\bullet}, \mathcal{D}_{X}^{\bullet}\right) \simeq \mathcal{D}_{X}^{\bullet} \otimes_{\mathcal{O}_{X}} \mathcal{H o m}_{X \text {-qc }}\left(\mathcal{E}_{\bullet}, \mathcal{O}_{X}\right)$, so the cone of the corresponding morphism of the total matrix factorizations is coacyclic.

2.6. w-flat matrix factorizations. From now on we will assume that for any affine open subscheme $U \subset X$ the element $\left.w\right|_{U}$ is not a zero divisor in the $\mathcal{O}(U)$-module $\mathcal{L}(U)$; in other words, the morphism of sheaves $w: \mathcal{O}_{X} \rightarrow \mathcal{L}$ is injective.

The following results will be used in the proof of the main theorem and its analogues below. Let us call a quasicoherent $\mathcal{O}_{X}$-module $\mathcal{E} w$-flat if the map $w: \mathcal{E} \rightarrow \mathcal{E} \otimes_{\mathcal{O}_{X}} \mathcal{L}$ is injective. Notice that any submodule of a $w$-flat module is $w$-flat, so the " $w$-flat dimension" of a quasicoherent sheaf over $X$ never exceeds 1 .

Denote by $(X, \mathcal{L}, w)$-coh ${ }_{w-\text { fl }}$ the DG-category of coherent CDG-modules over $(X, \mathcal{L}, w)$ with $w$-flat underlying graded $\mathcal{O}_{X}$-modules and by $(X, \mathcal{L}, w)$-qcoh ${ }_{w \text {-fl }}$ the similar DG-category of quasicoherent CDG-modules. Let $\mathrm{D}^{\text {abs }}\left((X, \mathcal{L}, w)-\operatorname{coh}_{w \text {-fl }}\right)$, $\mathrm{D}^{\text {abs }}\left((X, \mathcal{L}, w)\right.$-qcoh ${ }_{w \text {-fl }}$, and $\mathrm{D}^{\mathrm{co}}\left((X, \mathcal{L}, w)\right.$-qcoh $\left._{w \text {-fl }}\right)$ denote the corresponding derived categories of the second kind.

Furthermore, denote by $(X, \mathcal{L}, w)$-coh ${ }_{w \text {-finffd }}$ the DG-category of coherent CDGmodules over $(X, \mathcal{L}, w)$ whose underlying graded $\mathcal{O}_{X}$-modules are both $w$-flat and of finite flat dimension, and by $(X, \mathcal{L}, w)$-qcoh ${ }_{w \text {-finlfd }}$ the DG-category of $w$-flat quasicoherent CDG-modules of finite locally free dimension. Let the corresponding exotic derived categories be denoted by $\mathrm{D}^{\text {abs }}\left((X, \mathcal{L}, w)\right.$-coh $\left.{ }_{w \text {-finffd }}\right)$,

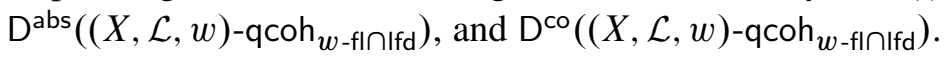

Corollary 2.6. (a) The functor $\mathrm{D}^{\mathrm{co}}\left((X, \mathcal{L}, w)\right.$-qcoh $\left.{ }_{w \text {-fl }}\right) \rightarrow \mathrm{D}^{\mathrm{co}}((X, \mathcal{L}, w)$-qcoh) induced by the embedding of DG-categories $(X, \mathcal{L}, w)$-qcoh $_{w-f l} \rightarrow(X, \mathcal{L}, w)$-qcoh is an equivalence of triangulated categories.

(b) The functor $\mathrm{D}^{\mathrm{abs}}\left((X, \mathcal{L}, w)\right.$-qcoh $\left.{ }_{w-\mathrm{fl}}\right) \rightarrow \mathrm{D}^{\mathrm{abs}}((X, \mathcal{L}, w)$-qcoh) induced by the embedding of DG-categories $(X, \mathcal{L}, w)$-qcoh $_{w-\mathrm{fl}} \rightarrow(X, \mathcal{L}, w)$-qcoh is an equivalence of triangulated categories.

(c) The functor $\mathrm{D}^{\mathrm{abs}}\left((X, \mathcal{L}, w)-\operatorname{coh}_{w-\mathrm{fl}}\right) \rightarrow \mathrm{D}^{\mathrm{abs}}((X, \mathcal{L}, w)$-coh) induced by the embedding of $D G$-categories $(X, \mathcal{L}, w)$-coh ${ }_{w-f l} \rightarrow(X, \mathcal{L}, w)$-coh is an equivalence of triangulated categories.

(d) The functor $\mathrm{D}^{\mathrm{co}}\left((X, \mathcal{L}, w)\right.$-qcoh $\left.{ }_{w-\mathrm{fl} \cap \mathrm{ffd}}\right) \rightarrow \mathrm{D}^{\mathrm{co}}\left((X, \mathcal{L}, w)\right.$-qcoh $\left.\mathrm{Ifd}_{1}\right)$ induced by the embedding of DG-categories $(X, \mathcal{L}, w)$-qcoh ${ }_{w \text {-fln Ifd }} \rightarrow(X, \mathcal{L}, w)$-qcoh ${ }_{\text {Ifd }}$ is an equivalence of triangulated categories. 
(e) The functor $\mathrm{D}^{\mathrm{abs}}\left((X, \mathcal{L}, w)\right.$-qcoh $\left.{ }_{w \text {-fIn Ifd }}\right) \rightarrow \mathrm{D}^{\mathrm{abs}}\left((X, \mathcal{L}, w)\right.$-qcoh $\left.\mathrm{Ifd}_{\mathrm{fd}}\right)$ induced by the embedding of DG-categories $(X, \mathcal{L}, w)$-qcoh ${ }_{w \text {-fln Ifd }} \rightarrow(X, \mathcal{L}, w)$-qcoh ${ }_{\mathrm{Ifd}}$ is an equivalence of triangulated categories.

(f) The functor $\mathrm{D}^{\mathrm{abs}}((X, \mathcal{L}, w)$-coh $w$-finffd $) \rightarrow \mathrm{D}^{\mathrm{abs}}\left((X, \mathcal{L}, w)\right.$-coh ffd $\left._{\text {ff }}\right)$ induced by the embedding of DG-categories $(X, \mathcal{L}, w)$-coh $_{w \text {-finffd }} \rightarrow(X, \mathcal{L}, w)$-coh $\operatorname{cff}_{\text {is }}$ is equivalence of triangulated categories.

Proof. The proofs are analogous to those of Corollary 2.3(a)-(c) and (g) (except that no induction in $d$ is needed, as it suffices to consider the case $d=1$ ). Parts (d), (e), (f) are analogous to parts (a), (b), (c), respectively. Parts (b), (c), (e), and (f) can be also proven in the way similar to Corollary 2.3(h) and (i).

Remark 2.6. The assertions of parts (a) and (b) hold under somewhat weaker assumptions than above: namely, one does not need to assume the existence of enough vector bundles on $X$. And one can make parts (d) and (e) hold without vector bundles by replacing the finite locally free dimension condition in their formulation with the finite flat dimension condition. The reason is that there are enough flat sheaves on any reasonable scheme (see Lemma A.1).

In fact, even part (c) does not depend on the existence of vector bundles since a surjective morphism onto a given coherent sheaf $\mathcal{M}$ from a $w$-flat coherent sheaf can be easily constructed, e.g., by starting from a surjective morphism onto $\mathcal{M}$ from a flat quasicoherent sheaf $\mathcal{F}$ and picking a large enough coherent subsheaf in $\mathcal{F}$. Accordingly, one does not need vector bundles to prove the equivalence of categories in the lower horizontal line in Theorem 2.7 below and the other two equivalences in Theorem 2.8. Replacing locally free sheaves with flat ones in the relevant definitions and assuming the Krull dimension to be finite, one can have the whole of Proposition 2.8 hold without vector bundles as well.

Another alternative is to use very flat quasicoherent sheaves, which there are always enough of and which always form a category of finite homological dimension on a quasicompact semiseparated scheme [Positselski 2012, Section 4.1]. Similarly, the existence of vector bundles is not needed for the validity of Theorem 1.4(a) and (b), Proposition 1.5(a), (c), and (d), all the assertions of Sections 1.7 and 1.10, Corollary 2.3(a), (b), (f), (k), and (1), Corollary 2.4(a) and (b), Proposition 2.5, Theorem 2.5, and some other results.

2.7. Main theorem. Let $X_{0} \subset X$ be the closed subscheme defined locally by the equation $w=0$, and $i: X_{0} \rightarrow X$ be the natural closed embedding. The next theorem is the main result of this paper.

Theorem 2.7. There is a natural equivalence of triangulated categories

$$
\mathrm{D}^{\text {abs }}((X, \mathcal{L}, w)-\text { coh }) \simeq \mathrm{D}_{\text {Sing }}^{\mathrm{b}}\left(X_{0} / X\right) .
$$


Together with the functor $\Sigma: \mathrm{D}^{\mathrm{abs}}\left((X, \mathcal{L}, w)\right.$-coh $\left.\mathrm{cof}_{\mathrm{f}}\right) \rightarrow \mathrm{D}_{\text {Sing }}^{\mathrm{b}}\left(X_{0}\right)$ constructed in [Orlov 2012], this equivalence forms the following diagram of triangulated functors:

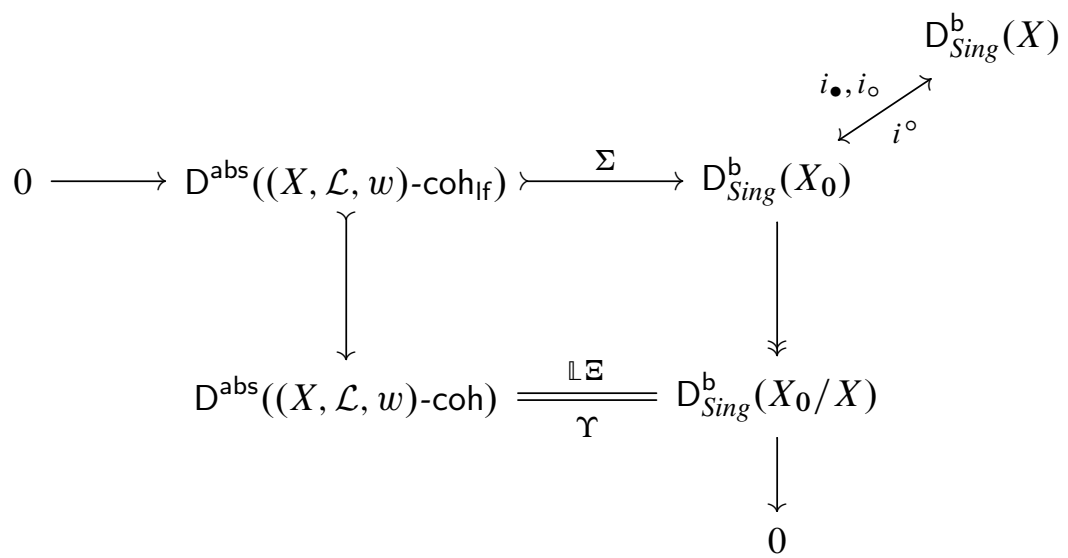

where the upper horizontal arrow $\Sigma$ is fully faithful, the left vertical arrow is fully faithful, the right vertical arrow is a Verdier localization functor, and the lower horizontal line $\mathbb{L} \Xi=\Upsilon^{-1}$ is an equivalence of categories. The square is commutative; the three diagonal arrows $i_{\bullet}, i^{\circ}, i_{\circ}$ (the middle one pointing down and the two other ones pointing up) are adjoint.

Furthermore, the image of the functor $\Sigma$ is precisely the full subcategory of objects annihilated by the functor $i_{\circ}$, or equivalently, by the functor $i_{\text {. }}$ In other words, the image of $\Sigma$ is equal both to the left and to the right orthogonal complements to the thick subcategory generated by the image of the functor $i^{\circ}$; that is, an object $\mathcal{F} \in \mathrm{D}_{\text {Sing }}^{\mathrm{b}}\left(X_{0}\right)$ is isomorphic to $\Sigma(\mathcal{M})$ for some $\mathcal{M} \in \mathrm{D}^{\mathrm{abs}}\left((X, \mathcal{L}, w)\right.$-coh $\left.\mathrm{co}_{\mathrm{If}}\right)$ if and only if for every $\mathcal{E} \in \mathrm{D}_{\text {Sing }}^{\mathrm{b}}(X)$, one has

$$
\operatorname{Hom}_{\mathrm{D}_{\text {Sing }}^{\mathrm{b}}\left(X_{0}\right)}\left(i^{\circ} \mathcal{E}, \mathcal{F}\right)=0,
$$

or equivalently, for every $\mathcal{E} \in \mathrm{D}_{\text {Sing }}^{\mathrm{b}}(X)$, one has $\operatorname{Hom}_{\mathrm{D}_{\text {Sing }}^{\mathrm{b}}\left(X_{0}\right)}\left(\mathcal{F}, i^{\circ} \mathcal{E}\right)=0$.

The thick subcategory generated by the image of the functor $i^{\circ}$ is the kernel of the right vertical arrow. So the upper horizontal arrow and the right vertical arrow are included into "exact sequences" of triangulated categories (as marked by the zeros at the ends; there is no exactness at the uppermost rightmost end).

When $X$ is a regular scheme, the functor

$$
\mathrm{D}^{\text {abs }}\left((X, \mathcal{L}, w)-\text { coh }_{\text {If }}\right) \longrightarrow \mathrm{D}^{\text {abs }}((X, \mathcal{L}, w) \text {-coh })
$$

is an equivalence of categories by Corollary 2.4(c), and so is the functor $D_{\text {Sing }}^{\mathrm{b}}\left(X_{0}\right) \rightarrow$ $\mathrm{D}_{\text {Sing }}^{\mathrm{b}}\left(X_{0} / X\right)$ (as explained in Section 2.1). Hence it follows that the functor $\Sigma$ is 
an equivalence of categories, too. Thus we recover the result of Orlov [2012, Theorem 3.5] claiming the equivalence of triangulated categories $\mathrm{D}^{\text {abs }}\left((X, \mathcal{L}, w)-\operatorname{coh}_{\text {lf }}\right) \simeq$ $\mathrm{D}_{\text {Sing }}^{\mathrm{b}}\left(X_{0}\right)$ for a regular $X$.

A counterexample in Section 3.3 will show that when $X$ is not regular, the functor $\mathrm{D}^{\text {abs }}((X, \mathcal{L}, w)$-coh If $) \rightarrow \mathrm{D}^{\text {abs }}((X, \mathcal{L}, w)$-coh $)$ does not have to be an equivalence, and indeed, the thick subcategory generated by $\mathrm{D}^{\mathrm{abs}}\left((X, \mathcal{L}, w)-\operatorname{coh}_{\mathrm{lf}}\right)$ can be a proper strictly full subcategory in $\mathrm{D}^{\text {abs }}((X, \mathcal{L}, w)$-coh).

Proof of the lower horizontal equivalence. To obtain the equivalence of triangulated categories in the lower horizontal line, we will construct triangulated functors in both directions and then check that they are mutually inverse. Given a bounded complex of coherent sheaves $\mathcal{F}^{\bullet}$ over $X_{0}$, consider the CDG-module $\Upsilon\left(\mathcal{F}^{\bullet}\right)$ over $(X, \mathcal{L}, w)$ with the underlying coherent graded module given by the rule

$$
\Upsilon^{n}\left(\mathcal{F}^{\bullet}\right)=\bigoplus_{m \in \mathbb{Z}} i_{*} \mathcal{F}^{n-2 m} \otimes_{\mathcal{O}_{X}} \mathcal{L}^{\otimes m}
$$

and the differential induced by the differential on $\mathcal{F}^{\bullet}$. Since $d^{2}=0$ on $\mathcal{F}^{\bullet}$ and $w$ acts by zero in $i_{*} \mathcal{F}^{j}$, this is a CDG-module. It is clear that $\Upsilon$ is a well-defined triangulated functor $\mathrm{D}^{\mathrm{b}}\left(X_{0}\right.$-coh $) \rightarrow \mathrm{D}^{\text {abs }}((X, \mathcal{L}, w)$-coh) since the derived category of bounded complexes over an abelian category coincides with their absolute derived category.

Let us check that $\Upsilon$ annihilates the image of the functor $\mathbb{L} i^{*}$. It suffices to consider a $w$-flat coherent sheaf $\mathcal{E}$ on $X$ and check that $\Upsilon(\operatorname{coker} w)=0$, where $w: \mathcal{E} \otimes_{\mathcal{O}_{X}} \mathcal{L}^{\otimes-1} \rightarrow \mathcal{E}$. Indeed, $\Upsilon($ coker $w)$ is the cokernel of the injective morphism of contractible coherent CDG-modules $\mathcal{N} \rightarrow \mathcal{M}$, where $\mathcal{N}^{2 n+1}=\mathcal{M}^{2 n+1}=$ $\mathcal{E} \otimes_{\mathcal{O}_{X}} \mathcal{L}^{\otimes n}$ and $\mathcal{N}^{2 n}=\mathcal{E} \otimes_{\mathcal{O}_{X}} \mathcal{L}^{\otimes n-1}$, while $\mathcal{M}^{2 n}=\mathcal{E} \otimes_{\mathcal{O}_{X}} \mathcal{L}^{\otimes n}$ for $n \in \mathbb{Z}$.

This provides the desired triangulated functor

$$
\Upsilon: \mathrm{D}_{\text {Sing }}^{\mathrm{b}}\left(X_{0} / X\right) \longrightarrow \mathrm{D}^{\mathrm{abs}}((X, \mathcal{L}, w)-\mathrm{coh}) .
$$

The functor in the opposite direction is a version of Orlov's cokernel functor, but in our situation it has to be constructed as a derived functor since the functor of the cokernel of an arbitrary morphism is not exact. Recall the equivalence of triangulated categories $\mathrm{D}^{\text {abs }}((X, \mathcal{L}, w)$-coh $w$-fl $) \rightarrow \mathrm{D}^{\text {abs }}((X, \mathcal{L}, w)$-coh) from Corollary 2.6(c).

Define the functor $\Xi: Z^{0}((X, \mathcal{L}, w)$-coh $w$-fl $) \rightarrow \mathrm{D}_{\text {Sing }}^{\mathrm{b}}\left(X_{0} / X\right)$ from the category of $w$-flat coherent CDG-modules over $(X, \mathcal{L}, w)$ and closed morphisms of degree 0 between them to the triangulated category of relative singularities by the rule

$$
\Xi(\mathcal{M})=\operatorname{coker}\left(d: \mathcal{M}^{-1} \rightarrow \mathcal{M}^{0}\right)=\operatorname{coker}\left(i^{*} d: i^{*} \mathcal{M}^{-1} \rightarrow i^{*} \mathcal{M}^{0}\right),
$$

where the former cokernel, which is by definition a coherent sheaf on $X$ annihilated by $w$, is considered as a coherent sheaf on $X_{0}$. One can immediately see that the functor $\Xi$ transforms morphisms homotopic to zero into morphisms factorizable 
through the restrictions to $X_{0}$ of $w$-flat coherent sheaves on $X$. Hence the functor $\Xi$ factorizes through the homotopy category $H^{0}\left((X, \mathcal{L}, w)-\operatorname{coh}_{w \text {-fl }}\right)$.

It is explained in [Polishchuk and Vaintrob 2011, Lemma 3.12] that the functor $\Xi$ is triangulated (see also Lemma 3.6 below) and in [Orlov 2012, Proposition 3.2] that the functor $\Xi$ factorizes through $\mathrm{D}^{\text {abs }}\left((X, \mathcal{L}, w)-\operatorname{coh}_{w \text {-fl }}\right)$. The latter assertion can be also deduced by considering the complex (1.3) from [Polishchuk and Vaintrob 2011]. Indeed, the complex $i^{*} \mathcal{M}$ corresponding to the total CDG-module $\mathcal{M}$ of an exact triple in $\mathcal{B}$-coh $w$-fl is the total complex of an exact triple of complexes in the exact category $\mathrm{E}_{X_{0} / X}$ from Remark 2.1; hence the complex $i^{*} \mathcal{M}$ is exact with respect to $\mathrm{E}_{X_{0} / X}$ and the cokernels of its differentials belong to this exact subcategory in the abelian category of coherent sheaves over $X_{0}$. So we obtain the triangulated functor

$$
\Xi: \mathrm{D}^{\mathrm{abs}}\left((X, \mathcal{L}, w)-\operatorname{coh}_{w-\mathrm{fl}}\right) \longrightarrow \mathrm{D}_{\text {Sing }}^{\mathrm{b}}\left(X_{0} / X\right),
$$

and consequently, the left derived functor

$$
\mathbb{L} \Xi: \mathrm{D}^{\mathrm{abs}}((X, \mathcal{L}, w)-\mathrm{coh}) \longrightarrow \mathrm{D}_{\text {Sing }}^{\mathrm{b}}\left(X_{0} / X\right) .
$$

Let us check that the two functors $\Upsilon$ and $\mathbb{E} \Xi$ are mutually inverse. For any $w$-flat coherent CDG-module $\mathcal{M}$ over $(X, \mathcal{L}, w)$, there is a natural surjective closed morphism of CDG-modules $\phi: \mathcal{M} \rightarrow \Upsilon \Xi(\mathcal{M})$ with a contractible kernel. Clearly, $\phi: \operatorname{Id} \rightarrow \Upsilon \mathbb{L} \Xi$ is an (iso)morphism of functors.

Conversely, any object of $D_{\text {Sing }}^{\mathrm{b}}\left(X_{0} / X\right)$ can be represented by a coherent sheaf on $X_{0}$, and any morphism in $\mathrm{D}_{\text {Sing }}^{\mathrm{b}}\left(X_{0} / X\right)$ is isomorphic to a morphism coming from the abelian category of such coherent sheaves. Indeed, the bounded-above derived category $\mathrm{D}^{-}\left(X_{0}\right.$-coh $)$ of coherent sheaves over $X_{0}$ is equivalent to the boundedabove derived category $\mathrm{D}^{-}\left(X_{0}-\mathrm{coh}_{\mathrm{lf}}\right)$ of locally free sheaves; using a truncation far enough to the left, one can represent any object or morphism in $D_{\text {Sing }}^{\mathrm{b}}\left(X_{0} / X\right)$ by a long enough shift of a coherent sheaf or a morphism of coherent sheaves. Now for any coherent sheaf $\mathcal{F}$ on $X_{0}$, there is a natural distinguished triangle

$$
\mathcal{F} \otimes_{\mathcal{O}_{X_{0}}} i^{*} \mathcal{L}^{\otimes-1}[1] \longrightarrow \mathbb{L} i^{*} i_{*} \mathcal{F} \longrightarrow \mathcal{F} \longrightarrow \mathcal{F} \otimes_{\mathcal{O}_{X_{0}}} i^{*} \mathcal{L}^{\otimes-1}[2]
$$

in $\mathrm{D}^{\mathrm{b}}\left(X_{0}\right.$-coh), which provides a natural isomorphism $\mathcal{F} \simeq \mathcal{F} \otimes_{\mathcal{O}_{X_{0}}} i^{*} \mathcal{L}^{\otimes-1}[2]$ in $\mathrm{D}_{\text {Sing }}^{\mathrm{b}}\left(X_{0} / X\right)$.

Let $\mathcal{F}$ be a coherent sheaf on $X_{0}$; pick a vector bundle $\mathcal{E}$ on $X$ together with a surjective morphism $\mathcal{E} \rightarrow i_{*} \mathcal{F}$ with the kernel $\mathcal{E}^{\prime}$. Then the CDG-module $\mathcal{M}$ over $(X, \mathcal{L}, w)$ with the components $\mathcal{M}^{2 n}=\mathcal{E} \otimes_{\mathcal{O}_{X}} \mathcal{L}^{\otimes n}$ and $\mathcal{M}^{2 n-1}=\mathcal{E}^{\prime} \otimes_{\mathcal{O}_{X}} \mathcal{L}^{\otimes n}$ maps surjectively onto $\Upsilon(\mathcal{F})$ with a contractible kernel, and $\mathbb{\Xi} \Upsilon(\mathcal{F})=\Xi(\mathcal{M})=\mathcal{F}$ (cf. [Lin and Pomerleano 2013, Lemma 2.18]). Denote the isomorphism we have constructed by $\psi: \mathbb{L} \Xi \Upsilon(\mathcal{F}) \rightarrow \mathcal{F}$. The composition $\Upsilon \psi \circ \phi \Upsilon: \Upsilon(\mathcal{F}) \rightarrow$ $\Upsilon \mathbb{\Xi} \Upsilon(\mathcal{F}) \rightarrow \Upsilon(\mathcal{F})$ is clearly the identity morphism. It is obvious that $\psi$ commutes 
with any morphism of coherent sheaves $\mathcal{F}$ on $X_{0}$, but checking that it commutes with all morphisms, or all isomorphisms, in $\mathrm{D}_{\text {Sing }}^{\mathrm{b}}\left(X_{0} / X\right)$ is a little delicate (cf. Remark 2.7 below).

Notice that $\Upsilon \psi$ is an (iso)morphism of functors since $\phi \Upsilon$ is, and consequently $\mathbb{L} \Xi \Upsilon \psi$ is an (iso)morphism of functors. Thus it remains to check that the functor $\mathbb{L} \Xi \Upsilon$ is faithful, i.e., does not annihilate any morphisms. Indeed, any morphism in $\mathrm{D}_{\text {Sing }}^{\mathrm{b}}\left(X_{0} / X\right)$ is isomorphic to a morphism coming from the abelian category of coherent sheaves on $X_{0}$, and the functor $\mathbb{L} \Xi \Upsilon$ transforms such morphisms into isomorphic ones. The construction of the equivalence of categories in the lower horizontal line is finished. One still has to check that the isomorphisms $\phi$ commute with the isomorphisms $\Upsilon \Xi(\mathcal{M}[1]) \simeq \Upsilon \Xi(\mathcal{M})[1]$, but this is straightforward.

Alternatively, one can use $w$-flat coherent sheaves on $X$ or objects of the exact category $\mathrm{E}_{X_{0} / X}$ of coherent sheaves on $X_{0}$ (as applicable) instead of the locally free sheaves everywhere in the above argument.

Proof of "exactness" in the upper line. We start with a discussion of the three adjoint functors in the right upper corner. The functor $i_{\circ}$ right adjoint to the functor $i^{\circ}: \mathrm{D}_{\text {Sing }}^{\mathrm{b}}(X) \rightarrow \mathrm{D}_{\text {Sing }}^{\mathrm{b}}\left(X_{0}\right)$ was constructed in Section 2.1.

To construct the left adjoint functor to $i^{\circ}$, notice that the right derived functor of the subsheaf with scheme-theoretic support in the closed subscheme

$$
\mathbb{R} i^{!}: \mathrm{D}^{\mathrm{b}}(X \text {-coh }) \longrightarrow \mathrm{D}^{\mathrm{b}}\left(X_{0} \text {-coh }\right)
$$

only differs from the functor $\mathbb{L} i^{*}$ by a shift and a twist; $\mathbb{R} i i^{\bullet} \mathcal{E}^{\bullet} \simeq \mathbb{L} i^{*} \mathcal{E}^{\bullet} \otimes_{\mathcal{O}_{X_{0}}}$ $\left.\mathcal{L}\right|_{X_{0}}[-1]$. One can check this first for $w$-flat coherent sheaves $\mathcal{E}$, when both objects to be identified are shifts of sheaves, so it suffices to compare their direct images under $i$, which are both computed by the same two-term complex $\mathcal{E} \rightarrow \mathcal{E} \otimes_{\mathcal{O}_{X}} \mathcal{L}$; then replace a complex $\mathcal{E}^{\bullet}$ with a finite complex of $w$-flat coherent sheaves (for a general result of this kind, see [Neeman 1996, Theorem 5.4]).

Hence the functor $\mathbb{R} i !$ takes $\operatorname{Perf}(X)$ to $\operatorname{Perf}\left(X_{0}\right)$ and induces a triangulated functor $i^{\bullet}: \mathrm{D}_{\text {Sing }}^{\mathrm{b}}(X) \rightarrow \mathrm{D}_{\text {Sing }}^{\mathrm{b}}\left(X_{0}\right)$ right adjoint to $i_{\circ}$. It follows that the functor $i_{\bullet}(\mathcal{F})=i_{\circ}(\mathcal{F}) \otimes_{\mathcal{O}_{X}} \mathcal{L}[-1]$ is left adjoint to the functor $i^{\circ}$.

To prove the vanishing of the composition of functors in the upper line and the orthogonality assertions, notice that

$$
\operatorname{Hom}_{\mathrm{D}_{\text {Sing }}^{\mathrm{b}}\left(X_{0}\right)}\left(i^{\circ} \mathcal{E}, \Sigma \mathcal{M}\right) \simeq \operatorname{Hom}_{\mathrm{D}_{\text {Sing }}^{\mathrm{b}}(X)}\left(\mathcal{E}, i_{\circ} \Sigma \mathcal{M}\right)
$$

and $i_{*} \Sigma(\mathcal{M})=\operatorname{coker}\left(\mathcal{M}^{-1} \rightarrow \mathcal{M}^{0}\right) \in \operatorname{Perf}(X)$ for any $\mathcal{M} \in \mathrm{D}^{\text {abs }}\left((X, \mathcal{L}, w)-\operatorname{coh}_{\text {If }}\right)$ since the morphism $\mathcal{M}^{-1} \rightarrow \mathcal{M}^{0}$ of locally free sheaves on $X$ is injective. Similarly,

$$
\operatorname{Hom}_{D_{\text {Sing }}^{\mathrm{b}}\left(X_{0}\right)}\left(\Sigma \mathcal{M}, i^{\circ} \mathcal{E}\right) \simeq \operatorname{Hom}_{D_{\text {Sing }}^{\mathrm{b}}(X)}\left(i_{\bullet} \Sigma \mathcal{M}, \mathcal{E}\right)
$$

and $i_{\bullet} \Sigma(\mathcal{M})=i_{\circ} \Sigma(\mathcal{M}) \otimes_{\mathcal{O}_{X}} \mathcal{L}[-1]=0$ in $\mathrm{D}_{\text {Sing }}^{\mathrm{b}}(X)$. 
Obviously, our derived cokernel functor $\mathbb{L} \Xi$ makes a commutative diagram with the cokernel functor $\Sigma$ from [Orlov 2012]. The left vertical arrow is fully faithful by Corollary 2.3(i). The assertion that the upper horizontal arrow is fully faithful is due to Orlov [2012, Theorem 3.4]. We have just obtained a new proof of it with our methods. Indeed, it follows from orthogonality that the functor $\mathrm{D}_{\text {Sing }}^{\mathrm{b}}\left(X_{0}\right) \rightarrow$ $\mathrm{D}_{\text {Sing }}^{\mathrm{b}}\left(X_{0} / X\right)$ induces isomorphisms on the groups of morphisms between any two objects, one of which comes from $\mathrm{D}^{\text {abs }}((X, \mathcal{L}, w)$-coh If $)$. Conversely, Orlov's theorem together with the orthogonality argument and the equivalence of categories in the lower horizontal line imply that the left vertical arrow is fully faithful.

Now assume that $i_{\circ} \mathcal{F}=0$ for some $\mathcal{F} \in \mathrm{D}_{\text {Sing }}^{\mathrm{b}}\left(X_{0}\right)$. Clearly, there exists $m \geq 0$ and a coherent sheaf $\mathcal{K}$ on $X_{0}$ such that $\mathcal{F} \simeq \mathcal{K}[m]$ in $D_{\text {Sing }}^{\mathrm{b}}\left(X_{0}\right)$. Then $i_{*} \mathcal{K}$ is a perfect complex, i.e., a coherent sheaf of finite flat dimension on $X$. Let us view it as an object of $(X, \mathcal{L}, w)$-coh ffd $_{\text {f }}$ i.e., consider the CDG-module $\mathcal{N}$ over $(X, \mathcal{L}, w)$ with the components $\mathcal{N}^{2 n}=i_{*} \mathcal{K} \otimes_{\mathcal{O}_{X}} \mathcal{L}^{\otimes n}$ and $\mathcal{N}^{2 n+1}=0$.

The construction of the cokernel functor $\Sigma$ can be straightforwardly extended to $w$-flat coherent matrix factorizations of finite flat dimension, providing a triangulated functor

$$
\widetilde{\Sigma}: \mathrm{D}^{\mathrm{abs}}\left((X, \mathcal{L}, w)-\operatorname{coh}_{w-\mathrm{fl} \cap \mathrm{ffd}}\right) \longrightarrow \mathrm{D}_{\text {Sing }}^{\mathrm{b}}\left(X_{0}\right) .
$$

The functor $\tilde{\Sigma}$ is well-defined since one has $i^{*} \mathcal{M} \in \operatorname{Perf}\left(X_{0}\right)$ for any $w$-flat coherent sheaf $\mathcal{M}$ of finite flat dimension on $X$. Using the equivalence of triangulated categories $\mathrm{D}^{\text {abs }}\left((X, \mathcal{L}, w)-\right.$ coh $\left._{w \text {-finffd }}\right) \simeq \mathrm{D}^{\text {abs }}\left((X, \mathcal{L}, w)\right.$-coh $\left.{ }_{\text {ffd }}\right)$ from Corollary 2.6(f), one constructs the derived functor

$$
\mathbb{L} \tilde{\Sigma}: \mathrm{D}^{\mathrm{abs}}\left((X, \mathcal{L}, w)-\text { coh }_{\mathrm{ffd}}\right) \longrightarrow \mathrm{D}_{\text {Sing }}^{\mathrm{b}}\left(X_{0}\right)
$$

in the same way as it was done above for the derived functor $\mathbb{L} \Xi$. Since the functor $\mathrm{D}^{\text {abs }}((X, \mathcal{L}, w)$-coh If $) \rightarrow \mathrm{D}^{\text {abs }}\left((X, \mathcal{L}, w)\right.$-coh $\left.{ }_{\mathrm{ffd}}\right)$ is an equivalence of categories by Corollary $2.3(\mathrm{~g})$, the (essential) images of the functors $\Sigma$ and $\mathbb{L} \widetilde{\Sigma}$ coincide.

Let us check that $\mathbb{L} \tilde{\Sigma}(\mathcal{N}) \simeq \mathcal{K}$ as an object of $D_{\text {Sing }}^{\mathrm{b}}\left(X_{0}\right)$. We argue as above, picking a vector bundle $\mathcal{E}$ on $X$ together with a surjective morphism $\mathcal{E} \rightarrow i_{*} \mathcal{K}$ with the kernel $\mathcal{E}^{\prime}$. Then the CDG-module $\mathcal{M}$ over $(X, \mathcal{L}, w)$ with the components $\mathcal{M}^{2 n}=\mathcal{E} \otimes_{\mathcal{O}_{X}} \mathcal{L}^{\otimes n}$ and $\mathcal{M}^{2 n-1}=\mathcal{E}^{\prime} \otimes{ }_{O_{X}} \mathcal{L}^{\otimes n}$ maps surjectively onto $\mathcal{N}$ with a contractible kernel. Hence the object $\mathcal{M} \in(X, \mathcal{L}, w)$ - $\operatorname{coh}_{w \text {-flnffd }}$ is isomorphic to $\mathcal{N}$ in $\mathrm{D}^{\text {abs }}\left((X, \mathcal{L}, w)\right.$-coh ffd $\left._{\text {ff }}\right)$, and we have $\mathbb{\Sigma} \widetilde{\Sigma}(\mathcal{N})=\widetilde{\Sigma}(\mathcal{M})=\mathcal{K}$. Therefore, the object $\mathcal{K} \in \mathrm{D}_{\text {Sing }}^{\mathrm{b}}\left(X_{0}\right)$ belongs to the (essential) image of the functor $\Sigma$, and it follows that so does the object $\mathcal{F} \simeq \mathcal{K}[m]$.

One can strengthen the above argument so as to obtain a construction of the (partial) inverse functor $\Delta$ to the functor $\Sigma$ similar to the above construction of the functor $\Upsilon$ inverse to the functor $\mathbb{\Xi}$. Consider the full subcategory $\mathrm{F}_{X_{0} / X} \subset X_{0}$-coh in the abelian category of coherent sheaves on $X_{0}$ consisting of all the sheaves $\mathcal{F}$ 
such that the sheaf $i_{*} \mathcal{F}$ has finite flat dimension (i.e., is a perfect complex) on $X$. The category $\mathrm{F}_{X_{0} / X}$ contains all the locally free sheaves on $X_{0}$ and is closed under the kernels of surjections, the cokernels of embeddings, and the extensions.

Hence $\mathrm{F}_{X_{0} / X}$ is an exact subcategory in $X_{0}$-coh. The natural functor

$$
\mathrm{D}^{\mathrm{b}}\left(\mathrm{F}_{X_{0} / X}\right) \longrightarrow \mathrm{D}^{\mathrm{b}}\left(X_{0}-\mathrm{coh}\right)
$$

is fully faithful; its image coincides with the kernel of the composition of the direct image and Verdier localization functors $\mathrm{D}^{\mathrm{b}}\left(X_{0}\right.$-coh $) \rightarrow \mathrm{D}^{\mathrm{b}}(X$-coh $) \rightarrow \mathrm{D}_{\text {Sing }}^{\mathrm{b}}(X)$. Accordingly, the quotient category $\mathrm{D}^{\mathrm{b}}\left(\mathrm{F}_{X_{0} / X}\right) / \mathrm{D}^{\mathrm{b}}\left(X_{0}\right.$-coh $\left.\mathrm{cof}_{\mathrm{f}}\right)$ is identified with the kernel of the direct image functor $i_{\circ}: \mathrm{D}_{\text {Sing }}^{\mathrm{b}}\left(X_{0}\right) \rightarrow \mathrm{D}_{\text {Sing }}^{\mathrm{b}}(X)$.

Now the functor

$$
\Delta: \mathrm{D}^{\mathrm{b}}\left(\mathrm{F}_{X_{0} / X}\right) / \mathrm{D}^{\mathrm{b}}\left(X_{0}-\operatorname{coh}_{\mathrm{lf}}\right) \longrightarrow \mathrm{D}^{\mathrm{abs}}\left((X, \mathcal{L}, w)-\operatorname{coh}_{\mathrm{ffd}}\right)
$$

is constructed in the way similar to the construction of the functor $\Upsilon$, by taking the direct image from $X_{0}$ to $X$ and applying the periodicity summation. That is,

$$
\Delta^{n}\left(\mathcal{F}^{\bullet}\right)=\bigoplus_{m \in \mathbb{Z}} i_{*} \mathcal{F}^{n-2 m} \otimes_{\mathcal{O}_{X}} \mathcal{L}^{\otimes m}
$$

for any $\mathcal{F}^{\bullet} \in \mathrm{D}^{\mathrm{b}}\left(\mathrm{F}_{X_{0} / X}\right)$. One checks that the functor $\Delta$ is inverse to the functor $\mathbb{\Sigma} \widetilde{\Sigma}$, the latter being viewed as a functor taking values in the triangulated subcategory $\mathrm{D}^{\mathrm{b}}\left(\mathrm{F}_{X_{0} / X}\right) / \mathrm{D}^{\mathrm{b}}\left(X_{0}\right.$-coh $\left.\mathrm{lf}\right) \subset \mathrm{D}_{\text {Sing }}^{\mathrm{b}}\left(X_{0}\right)$, in the same way as it was done above for the functors $\Upsilon$ and $\mathbb{\Xi}$. This provides yet another proof of the fact that the functor $\Sigma$ is fully faithful, together with another proof of our description of its image. It is also obvious from the constructions that the functor $\Delta$ makes a commutative diagram with the functor $\Upsilon$.

Remark 2.7. The somewhat tricky technical argument in the first part of the above proof can be clarified and generalized using the approach developed by the first author in [Efimov 2013, Appendix A].

Let $\mathrm{C}$ be an abelian category, $L: \mathrm{C} \rightarrow \mathrm{C}$ be its covariant autoequivalence, and $w: \operatorname{Id} \rightarrow L$ be a natural transformation commuting with $L$ (that is for any object $B \in \mathrm{C}$, one has $\left.w_{L(B)}=L\left(w_{B}\right)\right)$. Let $M F(\mathrm{C}, L, w)$ denote the abelian category of "matrix factorizations of $w$ in C", i.e., pairs of objects $U^{0}, L^{1 / 2}\left(U^{1}\right) \in C$ endowed with pairs of morphisms $U^{0} \rightarrow L^{1 / 2}\left(U^{1}\right), L^{1 / 2}\left(U^{1}\right) \rightarrow L\left(U^{0}\right)$ such that the compositions

$$
U^{0} \longrightarrow L^{1 / 2}\left(U^{1}\right) \longrightarrow L\left(U^{0}\right) \quad \text { and } \quad L^{1 / 2}\left(U^{1}\right) \longrightarrow L\left(U^{0}\right) \longrightarrow L^{3 / 2}\left(U^{1}\right)
$$

are equal to $w_{U^{0}}$ and $w_{L^{1 / 2}\left(U^{1}\right)}$, respectively. Given a matrix factorization $M=$ $\left(U^{0}, U^{1}\right)$, one sets

$$
M^{n}=L^{n / 2}\left(U^{n \bmod 2}\right) .
$$


Passing to the quotient category by the ideal of morphisms homotopic to zero, one obtains the homotopy category of matrix factorizations of $w$ in $\mathrm{C}$, and their absolute derived category, denoted by $\mathrm{D}^{\mathrm{abs}}(\mathrm{C}, L, w)$, is produced by the Verdier localization procedure similar to the one discussed in Section 1.3. (Cf. [Positselski 2011a, Remark 4.3].)

Let $\mathrm{C}_{0} \subset \mathrm{C}$ denote the full subcategory formed by all the objects $A \in \mathrm{C}$ for which $w_{A}=0$; so $C_{0}$ is an abelian subcategory in $C$ closed under subobjects and quotient objects. An object $B \in \mathrm{C}$ is said to have no $w$-torsion if the morphism $w_{B}$ is injective; and one says that the potential (natural transformation) $w$ does not divide zero in $C$ if every object of $C$ is the quotient object of an object without $w$-torsion. Let $i_{*}: \mathrm{C}_{0} \rightarrow \mathrm{C}$ denote the exact identity embedding functor and $i^{*}: \mathrm{C} \rightarrow \mathrm{C}_{0}$ be the functor left adjoint to $i_{*}$, so $i^{*}(B)=\operatorname{coker}\left(w_{L^{-1}(B)}: L^{-1}(B) \rightarrow B\right)$. Assuming that $w$ does not divide zero (as we do in the sequel), one can construct the left derived functor $\mathbb{L} i^{*}: \mathrm{D}^{\mathrm{b}}(\mathrm{C}) \rightarrow \mathrm{D}^{\mathrm{b}}\left(\mathrm{C}_{0}\right)$ with $\mathbb{L}_{s} i^{*}(B)=0$ for all $s \neq 0,1$ and any object $B \in \mathrm{C}$. The functor $\mathbb{L} i^{*}$ is left adjoint to the triangulated functor $i_{*}: \mathrm{D}^{\mathrm{b}}\left(\mathrm{C}_{0}\right) \rightarrow \mathrm{D}^{\mathrm{b}}(\mathrm{C})$ induced by the identity embedding $i_{*}: \mathrm{C}_{0} \rightarrow \mathrm{C}$.

Similarly, let $v^{n}: \mathrm{C}_{0} \rightarrow M F(\mathrm{C}, L, w)$ denote the exact functor assigning to an object $A \in \mathrm{C}_{0}$ the matrix factorization $M$ with $M^{n}=A$ and $M^{n+1}=0$, and let $\xi^{n}: M F(\mathrm{C}, L, w) \rightarrow \mathrm{C}_{0}$ be the functor left adjoint to $v^{n}$, assigning the object $\operatorname{coker}\left(M^{n-1} \rightarrow M^{n}\right) \in \mathrm{C}_{0}$ to a matrix factorization $M$. Considering the bounded derived category $\mathrm{D}^{\mathrm{b}} \operatorname{MF}(\mathrm{C}, L, w)$ of the abelian category $M F(\mathrm{C}, L, w)$, one can construct the left derived functor

$$
\llbracket \xi^{n}: \mathrm{D}^{\mathrm{b}} M F(\mathrm{C}, L, w) \longrightarrow \mathrm{D}^{\mathrm{b}}\left(\mathrm{C}_{0}\right) ;
$$

once again, the functor $\mathbb{L} \xi^{n}$ is left adjoint to $v^{n}: \mathrm{D}^{\mathrm{b}}\left(\mathrm{C}_{0}\right) \rightarrow \mathrm{D}^{\mathrm{b}} M F(\mathrm{C}, L, w)$ and one has $\mathbb{L}_{s} \xi^{n}(M)=0$ for all $s \neq 0,1$ and any matrix factorization $M \in M F(C, L, w)$.

Then the composition of the functor $v^{n}: \mathrm{D}^{\mathrm{b}}\left(\mathrm{C}_{0}\right) \rightarrow \mathrm{D}^{\mathrm{b}} M F(\mathrm{C}, L, w)$ with the totalization functor $\mathrm{D}^{\mathrm{b}} \operatorname{MF}(\mathrm{C}, L, w) \rightarrow \mathrm{D}^{\mathrm{abs}}(\mathrm{C}, L, w)$ induces an equivalence of triangulated categories

$$
\Upsilon^{n}: \mathrm{D}^{\mathrm{b}}\left(\mathrm{C}_{0}\right) /\left\langle\mathbb{L} i^{*} \mathrm{D}^{\mathrm{b}}(\mathrm{C})\right\rangle \longrightarrow \mathrm{D}^{\mathrm{abs}}(\mathrm{C}, L, w)
$$

between the quotient category of the derived category $D^{b}\left(C_{0}\right)$ by the thick subcategory generated by the image of the functor $\mathbb{L} i^{*}$ and the absolute derived category of matrix factorizations. The composition of the functor $\mathbb{L} \xi^{n}: \mathrm{D}^{\mathrm{b}} M F(\mathrm{C}, L, w) \rightarrow$ $\mathrm{D}^{\mathrm{b}}\left(\mathrm{C}_{0}\right)$ with the Verdier localization functor $\mathrm{D}^{\mathrm{b}}\left(\mathrm{C}_{0}\right) \rightarrow \mathrm{D}^{\mathrm{b}}\left(\mathrm{C}_{0}\right) /\left\langle\mathbb{L} i^{*} \mathrm{D}^{\mathrm{b}}(\mathrm{C})\right\rangle$ factorizes through the totalization functor $\mathrm{D}^{\mathrm{b}} M F(\mathrm{C}, L, w) \rightarrow \mathrm{D}^{\text {abs }}(\mathrm{C}, L, w)$, providing the triangulated functor

$$
\mathbb{L} \Xi^{n}: \mathrm{D}^{\mathrm{abs}}(\mathrm{C}, L, w) \longrightarrow \mathrm{D}^{\mathrm{b}}\left(\mathrm{C}_{0}\right) /\left\langle\mathbb{L} i^{*} \mathrm{D}^{\mathrm{b}}(\mathrm{C})\right\rangle
$$

inverse to $\Upsilon^{n}$. 
Indeed, let $F^{n}: M F(C, L, w) \rightarrow C$ denote the forgetful functor taking a matrix factorization $M$ to the object $M^{n} \in \mathrm{C}$, and let $G^{n-}: \mathrm{C} \rightarrow M F(\mathrm{C}, L, w)$ denote the functor left adjoint to $F^{n-1}$ (and right adjoint to $F^{n}$ ); so the functor $G^{n-}$ takes an object $B \in \mathrm{C}$ to a contractible matrix factorization $M$ with $M^{n-1}=M^{n}=B$ (cf. the constructions of the functors $G^{+}$and $G^{-}$in the proofs in Sections 1.4 and 1.6). It is claimed that the induced triangulated functors $G^{n-}: \mathrm{D}^{\mathrm{b}}(\mathrm{C}) \rightarrow \mathrm{D}^{\mathrm{b}} M F(\mathrm{C}, L, w)$ and $v^{n}: \mathrm{D}^{\mathrm{b}}\left(\mathrm{C}_{0}\right) \rightarrow \mathrm{D}^{\mathrm{b}} M F(\mathrm{C}, L, w)$ are fully faithful and their images form a semiorthogonal decomposition of the derived category $\mathrm{D}^{\mathrm{b}} M F(\mathrm{C}, L, w)$.

To check the first assertion, it suffices to notice that the triangulated functor $G^{n-}$ is left adjoint to the functor $F^{n-1}: \mathrm{D}^{\mathrm{b}} M F(\mathrm{C}, L, w) \rightarrow \mathrm{D}^{\mathrm{b}}(\mathrm{C})$, and their composition $F^{n-1} \circ G^{n-}$ is the identity endofunctor on $\mathrm{D}^{\mathrm{b}}(\mathrm{C})$. Similarly, the composition of triangulated functors $\llbracket \xi^{n} \circ v^{n}$ is the identity endofunctor on $\mathrm{D}^{\mathrm{b}}\left(\mathrm{C}_{0}\right)$, so $v^{n}$ is fully faithful as a functor between the derived categories. Furthermore, one has $F^{n-1} \circ v^{n}=0=\mathbb{L} \xi^{n} \circ G^{n-}$, implying the semiorthogonality. Finally, for any matrix factorization $M$ whose terms are objects without $w$-torsion, there is a short exact sequence

$$
0 \longrightarrow G^{n-} F(M) \longrightarrow M \longrightarrow v^{n} \xi^{n} M \longrightarrow 0
$$

in $M F(C, L, w)$ and $\mathbb{L} \xi^{n} M=\xi^{n} M$, proving the decomposition claim.

Now we notice that for any object $B \in \mathrm{C}$ having no $w$-torsion, there is a short exact sequence

$$
0 \longrightarrow G^{(n+2)-}(B) \longrightarrow G^{(n+1)-}(B) \longrightarrow v^{n} j^{*} B \longrightarrow 0
$$

in $M F(C, L, w)$. According to (the proof of) [Efimov 2013, Proposition A.3(1) and (2)], the totalization functor $\mathrm{D}^{\mathrm{b}} M F(\mathrm{C}, L, w) \rightarrow \mathrm{D}^{\text {abs }}(\mathrm{C}, L, w)$ is the Verdier localization functor by the thick subcategory generated by the objects of the form $G^{n-}(B)=G^{(n+2)-} L(B)$ and $G^{(n+1)-}(B) \in M F(C, L, w) \subset \mathrm{D}^{\mathrm{b}} M F(\mathrm{C}, L, w)$. The assertions about the existence of triangulated functors $\Upsilon^{n}$ and $\mathbb{L} \Xi^{n}$ and their being mutually inverse equivalences of categories follow from these observations.

Returning to a separated Noetherian scheme $X$ with enough vector bundles and the Cartier divisor $X_{0} \subset X$ of a global section $w$ of a line bundle $\mathcal{L}$ on $X$, the above approach based on [loc. cit., Proposition A.3] provides an elegant construction of Orlov's triangulated cokernel functor $\Sigma: \mathrm{D}^{\mathrm{abs}}\left((X, \mathcal{L}, w)\right.$-coh $\left.{ }_{\mathrm{lf}}\right) \rightarrow \mathrm{D}_{\text {Sing }}^{\mathrm{b}}\left(X_{0}\right)$ in addition to a proof of our equivalence of categories $\mathrm{D}^{\mathrm{abs}}((X, \mathcal{L}, w)$-coh $) \simeq \mathrm{D}_{\text {Sing }}^{\mathrm{b}}\left(X_{0} / X\right)$.

2.8. Infinite matrix factorizations. Following [Orlov 2004, paragraphs after Remark 1.9], one can define a "large" version of the triangulated category of singularities $\mathrm{D}_{\text {Sing }}^{\prime}(X)$ of a scheme $X$ as the quotient category of the bounded derived category of quasicoherent sheaves $\mathrm{D}^{\mathrm{b}}\left(X\right.$-qcoh) by the thick subcategory $\mathrm{D}^{\mathrm{b}}\left(X\right.$-qcoh $\left._{\text {If }}\right)$ of bounded complexes of locally free sheaves (of infinite rank). When $X$ has 
finite Krull dimension, the latter subcategory coincides with the thick subcategory $\mathrm{D}^{\mathrm{b}}\left(X\right.$-qcoh $\left._{\mathrm{fl}}\right)$ of bounded complexes of flat sheaves (see Remark 1.4).

Similarly, let $Z \subset X$ be a closed subscheme such that $\mathcal{O}_{Z}$ has finite flat dimension as an $\mathcal{O}_{X}$-module. Let us define a "large" triangulated category of relative singularities $\mathrm{D}_{\text {Sing }}^{\prime}(Z / X)$ as the quotient category of $D^{\mathrm{b}}(Z$-qcoh) by the minimal thick subcategory containing the image of the functor $\mathbb{L} i^{*}: \mathrm{D}^{\mathrm{b}}(X$-qcoh $) \rightarrow \mathrm{D}^{\mathrm{b}}(Z$-qcoh $)$ and closed under those infinite direct sums that exist in $D^{\mathrm{b}}(Z$-qcoh $)$. The quotient category of $\mathrm{D}^{\mathrm{b}}(Z$-qcoh $)$ by the minimal thick subcategory containing $\mathbb{L} i^{*} \mathrm{D}^{\mathrm{b}}(X$-qcoh $)$ (without the direct sum closure) will be also of interest to us; let us denote it by $\mathrm{D}_{\text {Sing }}^{\prime \prime}(Z / X)$.

Lemma 2.8. The triangulated categories $\mathrm{D}_{\text {Sing }}^{\prime}(Z / X)$ and $\mathrm{D}_{\text {Sing }}^{\prime \prime}(Z / X)$ are quotient categories of $\mathrm{D}_{\text {Sing }}^{\prime}(Z)$. When the scheme $X$ is regular of finite Krull dimension, these three triangulated categories coincide.

Proof. To prove the first assertion, let us show that any locally free sheaf on $Z$, considered as an object of $\mathrm{D}^{\mathrm{b}}(Z$-qcoh), is a direct summand of a bounded complex whose terms are direct sums of locally free sheaves of finite rank restricted from $X$. Indeed, pick a finite left resolution of a given locally free sheaf on $Z$ with the middle terms as above, long enough compared to the number of open subsets in an affine covering of $Z$. Then the corresponding Ext class between the cohomology sheaves at the rightmost and leftmost terms has to vanish in view of the Mayer-Vietoris sequence for Ext groups between quasicoherent sheaves [Orlov 2004, Lemma 1.12]. Hence the rightmost term is a direct summand of the complex formed by the middle terms.

The second assertion holds for the categories $\mathrm{D}_{\text {Sing }}^{\prime \prime}(Z / X)$ and $\mathrm{D}_{\text {Sing }}^{\prime}(Z)$ since any quasicoherent sheaf on a regular scheme of finite Krull dimension has a finite left resolution consisting of locally free sheaves. To identify these two categories with $\mathrm{D}_{\text {Sing }}^{\prime}(Z / X)$, one needs to know that the subcategory of bounded complexes of locally free sheaves on $Z$ is closed under those infinite direct sums that exist in $\mathrm{D}^{\mathrm{b}}(Z$-qcoh). The latter is true for any Noetherian scheme $Z$ of finite Krull dimension with enough vector bundles since the finitistic projective dimension of a commutative ring of finite Krull dimension is finite [Raynaud and Gruson 1971, Théorème II.3.2.6].

Now let $\mathcal{L}$ be a line bundle on $X, w \in \mathcal{L}(X)$ be a global section corresponding to an injective morphism of sheaves $\mathcal{O}_{X} \rightarrow \mathcal{L}$, and $X_{0} \subset X$ be the locus of $w=0$.

Proposition 2.8. There is a natural equivalence of triangulated categories

$$
\mathrm{D}^{\text {abs }}((X, \mathcal{L}, w) \text {-qcoh }) \simeq \mathrm{D}_{\text {Sing }}^{\prime \prime}\left(X_{0} / X\right) .
$$

Together with the infinite-rank version $\Sigma^{\prime}: \mathrm{D}^{\mathrm{abs}}\left((X, \mathcal{L}, w)\right.$-qcoh $\left.{ }_{\mathrm{lf}}\right) \rightarrow \mathrm{D}_{\text {Sing }}^{\prime}\left(X_{0}\right)$ of Orlov's cokernel functor $\Sigma$ from [Orlov 2012], this equivalence forms the following diagram of triangulated functors: 


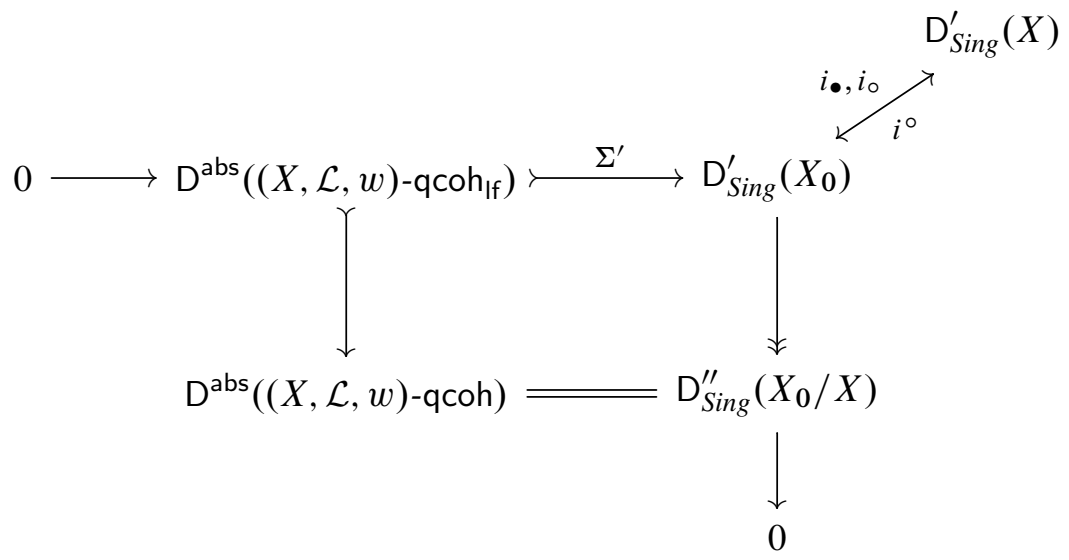

where the upper horizontal arrow $\Sigma^{\prime}$ is fully faithful, the left vertical arrow is fully faithful, the right vertical arrow is the Verdier localization functor by the thick subcategory generated by the image of the diagonal down arrow $i^{\circ}$, and the lower horizontal line is an equivalence of categories. The square is commutative; the three diagonal arrows $i_{\bullet}, i^{\circ}, i_{\circ}$ are adjoint.

Furthermore, the image of the functor $\Sigma^{\prime}$ is precisely the full subcategory of objects annihilated by the functor $i_{\circ}$, or equivalently, by the functor $i_{\text {. }}$. In other words, the image of $\Sigma^{\prime}$ is equal both to the left and to the right orthogonal complements to (the thick subcategory generated by) the image of the functor $i^{\circ}$.

Proof. The proof is completely similar to that of Theorem 2.7. It uses Corollaries 2.6(b), 2.3(h), 2.6(e), and 2.3(c). The first assertion can be also obtained as a particular case of the result of Remark 2.7.

Alternatively, one can prove that the functor $\Sigma^{\prime}$ is fully faithful in the same way as it was done for the functor $\Sigma$ in [Orlov 2012, Theorem 3.4], and deduce the assertion that the left vertical arrow is fully faithful from the orthogonality.

Note that one can check in a straightforward way that the functor $\Sigma^{\prime}$ annihilates the objects coacyclic with respect to $(X, \mathcal{L}, w)$-qcoh lf $_{\text {. This provides another proof }}$ of Corollary 2.3(d), working in the assumption that $w$ is a local nonzero-divisor.

The functors $\Sigma$ and $\Sigma^{\prime}$ together with the direct image functors $i_{\circ}$ form the commutative diagram of an embedding of "exact sequences" of triangulated functors:

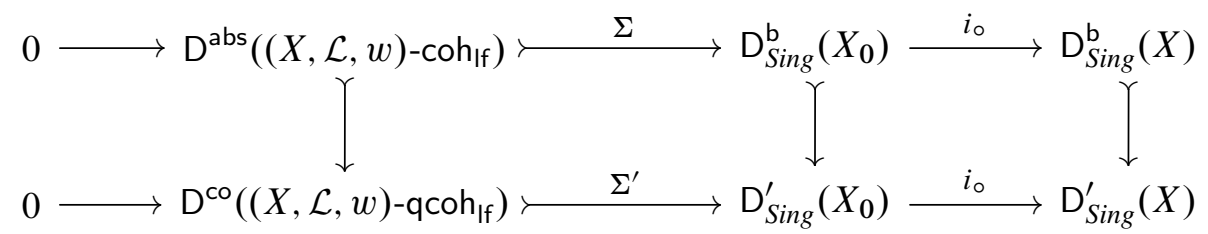

The leftmost vertical arrow is fully faithful by Corollary 2.3(j). The other two vertical arrows are fully faithful by Orlov's theorem [2004, Proposition 1.13] claiming 
that the functor $\mathrm{D}_{\text {Sing }}^{\mathrm{b}}(X) \rightarrow \mathrm{D}_{\text {Sing }}^{\prime}(X)$ is fully faithful for any separated Noetherian scheme $X$ with enough vector bundles. The leftmost nontrivial terms in both lines are the kernels of the rightmost arrows by Theorem 2.7 and Proposition 2.8.

Theorem 2.8. There is a natural equivalence of triangulated categories

$$
\mathrm{D}^{\mathrm{co}}((X, \mathcal{L}, w)-\mathrm{qcoh}) \simeq \mathrm{D}_{\text {Sing }}^{\prime}\left(X_{0} / X\right)
$$

forming a commutative diagram of triangulated functors:

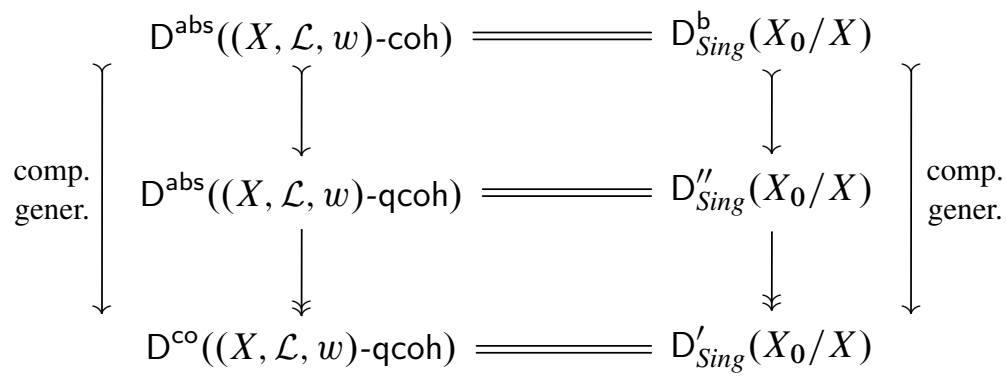

with the equivalences of categories from Theorem 2.7 and Proposition 2.8. The upper vertical arrows are fully faithful, the lower ones are Verdier localization functors, and the vertical compositions are fully faithful. The categories in the lower line admit arbitrary direct sums, and the images of the vertical compositions are sets of compact generators in the target categories.

Proof. The construction of the desired equivalence of categories is very similar to the construction of the equivalence of categories in Theorem 2.7 and Proposition 2.8. Using Corollary 2.6(a), one defines the infinite-rank version of the functor $\mathbb{L} \Xi$, then shows that the obvious infinite-rank version of the functor $\Upsilon$ is inverse to it. Notice that the functor $\Xi: Z^{0}\left((X, \mathcal{L}, w)\right.$-q $\left.\operatorname{coh}_{w \text {-fl }}\right) \rightarrow \mathrm{D}^{\mathrm{b}}\left(X_{0}\right.$-qcoh $)$ preserves infinite direct sums and the functor $\Upsilon: \mathrm{D}^{\mathrm{b}}\left(X_{0}\right.$-qcoh $) \rightarrow \mathrm{D}^{\mathrm{co}}((X, \mathcal{L}, w)$-qcoh $)$ preserves those infinite direct sums that exist in $D^{\mathrm{b}}\left(X_{0}\right.$-qcoh), so the functors

$$
\begin{gathered}
\Xi: \mathrm{D}^{\mathrm{co}}\left((X, \mathcal{L}, w)-\mathrm{qcoh}_{w-\mathrm{fl}}\right) \longrightarrow \mathrm{D}_{\text {Sing }}^{\prime}\left(X_{0} / X\right), \\
\Upsilon: \mathrm{D}_{\text {Sing }}^{\prime}\left(X_{0} / X\right) \longrightarrow \mathrm{D}^{\mathrm{co}}((X, \mathcal{L}, w) \text {-qcoh })
\end{gathered}
$$

are well-defined.

The upper left vertical arrow is fully faithful by Corollary $2.3(\mathrm{k})$; it follows that the upper right vertical arrow is fully faithful, too. The assertions about the vertical compositions are proved similarly. The category $\mathrm{D}_{\text {Sing }}^{\prime}\left(X_{0} / X\right)$ admits arbitrary direct sums, since the category $\mathrm{D}^{\mathrm{co}}((X, \mathcal{L}, w)$-qcoh) does. By Corollary $2.3(1)$, the left vertical composition is fully faithful and its image is a set of compact generators in the target, so the right vertical composition has the same properties. 
The following square diagram of triangulated functors is commutative:

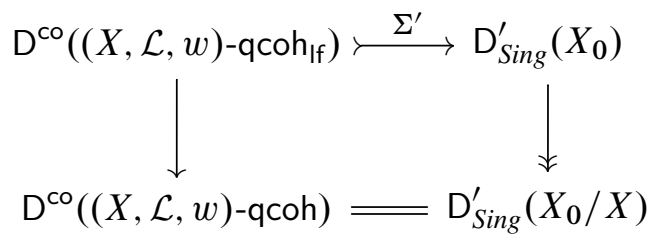

The upper horizontal arrow $\Sigma^{\prime}$ is fully faithful; the right vertical arrow is a Verdier localization functor. The lower line is an equivalence of triangulated categories. Nothing is claimed about the left vertical arrow in general.

When the scheme $X$ is Gorenstein of finite Krull dimension, the left vertical arrow is an equivalence of categories by Corollary 2.4(a). When $X$ is also regular, the right vertical arrow is an equivalence of categories by Lemma 2.8. So $\Sigma^{\prime}$ is an equivalence of categories $\mathrm{D}^{\text {abs }}\left((X, \mathcal{L}, w)\right.$-qcoh $\left.\mathrm{h}_{\mathrm{If}}\right) \simeq \mathrm{D}_{\text {Sing }}^{\prime}\left(X_{0}\right)$ and we have obtained a strengthened version of [Polishchuk and Vaintrob 2011, Theorem 4.2] (in the scheme case).

Remark 2.8. It is well-known that the Verdier localization functor of a triangulated category with infinite direct sums by a thick subcategory closed under infinite direct sums preserves infinite direct sums [Neeman 2001, Lemma 3.2.10]. This result is not applicable to the localization functors $\mathrm{D}^{\mathrm{b}}(X$-qcoh $) \rightarrow \mathrm{D}_{\text {Sing }}^{\prime}(X)$ and $\mathrm{D}^{\mathrm{b}}(Z$-qcoh $) \rightarrow \mathrm{D}_{\text {Sing }}^{\prime}(Z / X)$, as the category $\mathrm{D}^{\mathrm{b}}(X$-qcoh $)$ does not admit arbitrary infinite direct sums.

Using the equivalence of categories from Theorem 2.8 and the observation that the functor $\Upsilon$ preserves infinite direct sums, one can show that the localization functor $\mathrm{D}^{\mathrm{b}}\left(X_{0}\right.$-qcoh $) \rightarrow \mathrm{D}_{\text {Sing }}^{\prime}\left(X_{0} / X\right)$ takes those infinite direct sums that exist in $\mathrm{D}^{\mathrm{b}}\left(X_{0}\right.$-qcoh $)$ into direct sums in the triangulated category of relative singularities $\mathrm{D}_{\text {Sing }}^{\prime}\left(X_{0} / X\right)$ of the zero locus of $w$ in $X$. However, there is no obvious reason why the localization functor $\mathrm{D}^{\mathrm{b}}\left(X_{0}\right.$-qcoh $) \rightarrow \mathrm{D}_{\text {Sing }}^{\prime}\left(X_{0}\right)$ should take those infinite direct sums that exist in $\mathrm{D}^{\mathrm{b}}\left(X_{0}\right.$-qcoh) into direct sums in the absolute triangulated category of singularities $\mathrm{D}_{\text {Sing }}^{\prime}\left(X_{0}\right)$.

That is the problem one encounters attempting to prove that the kernel of the localization functor $\mathrm{D}_{\text {Sing }}^{\prime}\left(X_{0}\right) \rightarrow \mathrm{D}_{\text {Sing }}^{\prime}\left(X_{0} / X\right)$ is semiorthogonal to the image of the functor $\Sigma^{\prime}$.

2.9. Stable derived category. Following Krause [2005], we define the stable derived category of a Noetherian scheme $X$ as the homotopy category of acyclic unbounded complexes of injective quasicoherent sheaves on $X$. As explained below, this is another (and in some respects better) "large" version of the triangulated category of singularities of $X$; for this reason, we denote it by $\mathrm{D}_{\text {Sing }}^{\text {st }}(X)$. 
In view of Lemma 1.7(b) (see also [Positselski 2010, Remark 5.4]), one can equivalently define $\mathrm{D}_{\text {Sing }}^{\text {st }}(X)$ as the quotient category of the homotopy category of acyclic complexes of quasicoherent sheaves over $X$ by the thick subcategory of coacyclic complexes, or as the full subcategory of acyclic complexes in the coderived category $\mathrm{D}^{\mathrm{co}}(X$-qcoh) of (complexes of) quasicoherent sheaves over $X$. It is the latter definition that will be used in the sequel.

Clearly, the category $\mathrm{D}_{\text {Sing }}^{\text {st }}(X)$ has arbitrary infinite direct sums. Krause [2005, Corollary 5.4] constructs a fully faithful functor $\mathrm{D}_{\text {Sing }}^{\mathrm{b}}(X) \rightarrow \mathrm{D}_{\text {Sing }}^{\text {st }}(X)$ and proves that its image is a set of compact generators of the target category.

Theorem 2.9. For any separated Noetherian scheme $Z$ with enough vector bundles, there is a natural triangulated functor $\mathrm{D}_{\text {Sing }}^{\prime}(Z) \rightarrow \mathrm{D}_{\text {Sing }}^{\text {st }}(Z)$ forming a commutative diagram with the natural functors from $\mathrm{D}_{\text {Sing }}^{\mathrm{b}}(Z)$ into both these categories. The composition

$$
\mathrm{D}^{\mathrm{b}}(Z \text {-qcoh }) \longrightarrow \mathrm{D}_{\text {Sing }}^{\prime}(Z) \longrightarrow \mathrm{D}_{\text {Sing }}^{\mathrm{st}}(Z)
$$

preserves those infinite direct sums that exist in $\mathrm{D}^{\mathrm{b}}\left(Z\right.$-qcoh). When $Z=X_{0}$ is a divisor in a regular separated Noetherian scheme of finite Krull dimension, the functor $\mathrm{D}_{\text {Sing }}^{\prime}\left(X_{0}\right) \rightarrow \mathrm{D}_{\text {Sing }}^{\text {st }}\left(X_{0}\right)$ is an equivalence of triangulated categories.

Proof. The construction of the functor $D_{\text {Sing }}^{\mathrm{b}}(Z) \rightarrow \mathrm{D}_{\text {Sing }}^{\text {st }}(Z)$ in [Krause 2005] is given in terms of the Verdier localization functor $Q: \mathrm{D}^{\mathrm{co}}(Z$-qcoh $) \rightarrow \mathrm{D}(Z$-qcoh $)$ by the triangulated subcategory $\mathrm{D}_{\text {Sing }}^{\text {st }}(Z) \subset \mathrm{D}^{\mathrm{co}}(Z$-qcoh $)$ and its adjoint functors on both sides, which exist according to [loc. cit., Corollary 4.3]. The proof of our theorem is based on explicit constructions of the restrictions of these adjoint functors to some subcategories of bounded complexes in $\mathrm{D}(Z$-qcoh).

It is well known that the Verdier localization functor $H^{0}(Z$-qcoh $) \rightarrow \mathrm{D}(Z$-qcoh $)$ from the homotopy category of (complexes of) quasicoherent sheaves on $Z$ to their derived category has a right adjoint functor $\mathrm{D}(Z$-qcoh $) \rightarrow H^{0}(Z$-qcoh $)$. The objects in the image of this functor are called homotopy injective complexes of quasicoherent sheaves on $Z$. The composition $\mathrm{D}(Z$-qcoh $) \rightarrow H^{0}(Z$-qcoh $) \rightarrow \mathrm{D}^{\mathrm{co}}(Z$-qcoh $)$ provides the functor $Q_{\rho}: \mathrm{D}(Z$-qcoh $) \rightarrow \mathrm{D}^{\mathrm{co}}(Z$-qcoh $)$ right adjoint to $Q$. In particular, any bounded-below complex in $\mathrm{D}(Z$-qcoh) has a bounded-below injective resolution and any bounded-below complex of injectives is homotopy injective. Furthermore, any bounded-below acyclic complex is coacyclic [Positselski 2010, Lemma 2.1]. It follows that any bounded-below complex from $\mathrm{D}^{+}(Z$-qcoh $)$, considered as an object of $\mathrm{D}^{\mathrm{co}}\left(Z\right.$-qcoh), represents its own image under the functor $Q_{\rho}$.

On the other hand, any bounded-above complex from $\mathrm{D}(Z$-qcoh $)$ has a locally free left resolution defined uniquely up to a quasi-isomorphism of complexes in the exact category of locally free sheaves; i.e., there is an equivalence of bounded above derived categories $\mathrm{D}^{-}\left(Z\right.$-qcoh $\left.{ }_{\mathrm{lf}}\right) \simeq \mathrm{D}^{-}(Z$-qcoh $)$. Since the exact category $Z$-qcoh ${ }_{\text {lf }}$ has finite homological dimension, any acyclic complex in it is coacyclic 
(and even absolutely acyclic [loc. cit., Remark 2.1]), so there are natural functors $\mathrm{D}^{-}\left(Z\right.$-qcoh $\left.{ }_{\mathrm{lf}}\right) \rightarrow \mathrm{D}(Z$-qcoh $\mathrm{lf}) \simeq \mathrm{D}^{\mathrm{co}}(Z$-qcoh lf $) \rightarrow \mathrm{D}^{\mathrm{co}}(Z$-qcoh $)$.

Lemma 2.9. The composition of the embedding $\mathrm{D}^{-}(Z$-qcoh $) \rightarrow \mathrm{D}(Z$-qcoh $)$ with the functor $Q_{\lambda}: \mathrm{D}(Z$-qcoh $) \rightarrow \mathrm{D}^{\mathrm{co}}(Z$-qcoh $)$ left adjoint to $Q$ is isomorphic to the functor $\mathrm{D}^{-}(Z$-qcoh $) \rightarrow \mathrm{D}^{\mathrm{co}}(Z$-qcoh $)$ constructed above.

Proof. We have to show that $\operatorname{Hom}_{\mathrm{D}^{\text {co }}(Z \text {-qcoh })}\left(\mathcal{L}^{\bullet}, \mathcal{E}^{\bullet}\right)=0$ for any bounded-above complex of locally free sheaves $\mathcal{L}^{\bullet}$ and any acyclic complex $\mathcal{E}^{\bullet}$ of quasicoherent sheaves on $Z$. Let us check that any morphism $\mathcal{L}^{\bullet} \rightarrow \mathcal{E}^{\bullet}$ in $H^{0}(Z$-qcoh) factorizes through a coacyclic complex of quasicoherent sheaves. Clearly, we can assume that the complex $\mathcal{E}^{\bullet}$ is bounded above, too. Let $\mathcal{K}^{\bullet}$ be the cocone of a closed morphism of complexes $\mathcal{L}^{\bullet} \rightarrow \mathcal{E}^{\bullet}$; then $\mathcal{K}^{\bullet}$ is bounded above and the composition $\mathcal{K}^{\bullet} \rightarrow \mathcal{L}^{\bullet} \rightarrow \mathcal{E}^{\bullet}$ is homotopic to zero. Pick a bounded-above complex of locally free sheaves $\mathcal{F}^{\bullet}$ together with a quasi-isomorphism $\mathcal{F}^{\bullet} \rightarrow \mathcal{K}^{\bullet}$. Then the cone of the composition $\mathcal{F}^{\bullet} \rightarrow \mathcal{K}^{\bullet} \rightarrow \mathcal{L}^{\bullet}$, being a bounded-above acyclic complex of locally free sheaves, is coacyclic. Since the composition $\mathcal{F}^{\bullet} \rightarrow \mathcal{L}^{\bullet} \rightarrow \mathcal{E}^{\bullet}$ is homotopic to zero, the morphism $\mathcal{L}^{\bullet} \rightarrow \mathcal{E}^{\bullet}$ factorizes, up to homotopy, through this cone.

Now we can describe the action of the functor $I_{\lambda}: \mathrm{D}^{\mathrm{co}}(Z$-qcoh $) \rightarrow \mathrm{D}_{\text {Sing }}^{\text {st }}(Z$-qcoh $)$ left adjoint to the embedding $\mathrm{D}_{\text {Sing }}^{\text {st }}(Z$-qcoh $) \rightarrow \mathrm{D}^{\mathrm{co}}(Z$-qcoh $)$ on bounded-above complexes in $\mathrm{D}^{\mathrm{co}}(Z$-qcoh $)$. If $\mathcal{K}^{\bullet}$ is a bounded-above complex of quasicoherent sheaves and $\mathcal{F}^{\bullet}$ is its locally free left resolution, then the cone of the closed morphism $\mathcal{F}^{\bullet} \rightarrow \mathcal{K}^{\bullet}$ represents the object $I_{\lambda}\left(\mathcal{K}^{\bullet}\right) \in \mathrm{D}_{\text {Sing }}^{\text {st }}(Z$-qcoh $)$. In view of Lemma 2.9, this cone is functorial and does not depend on the choice of $\mathcal{F}^{\bullet}$ for the usual semiorthogonality reasons.

The embedding of compact generators $D_{\text {Sing }}^{\mathrm{b}}(Z) \rightarrow \mathrm{D}_{\text {Sing }}^{\text {st }}(Z)$ is constructed in [Krause 2005] as the functor induced by the restriction of the composition $I_{\lambda} \circ Q_{\rho}: \mathrm{D}(Z$-qcoh $) \rightarrow \mathrm{D}_{\text {Sing }}^{\text {st }}(Z)$ to the full subcategory $\mathrm{D}^{\mathrm{b}}(Z$-coh $) \subset \mathrm{D}(Z$-qcoh $)$. Let us explain why this is so. By Proposition 1.5(d) (cf. [loc. cit., Proposition 2.3 and Remark 3.8]), the natural functor $D^{b}(Z$-coh $) \rightarrow D^{c o}(Z$-qcoh $)$ is fully faithful and its image is a set of compact generators in the target. This is the image of $\mathrm{D}^{\mathrm{b}}(Z$-coh $) \subset \mathrm{D}(Z$-qcoh $)$ under the functor $Q_{\rho}$, as constructed above. It is clear from the above construction of the functor $Q_{\lambda}$ that it preserves compactness (and in fact coincides with the functor $Q_{\rho}$ on perfect complexes in $\mathrm{D}(Z$-qcoh) [loc. cit., Lemma 5.2]). Since the functors $Q_{\lambda}$ and $I_{\lambda}$, being left adjoints, preserve infinite direct sums, and $I_{\lambda}$ is a Verdier localization functor by the image of $Q_{\lambda}$, it follows that the image of any set of compact generators of $\mathrm{D}^{\mathrm{co}}(Z$-qcoh $)$ under $I_{\lambda}$ is a set of compact generators of $D_{\text {Sing }}^{\text {st }}(Z)$ [Neeman 1996, Theorem 2.1(4)].

In order to define the desired functor

$$
\mathrm{D}_{\text {Sing }}^{\prime}(Z) \longrightarrow \mathrm{D}_{\text {Sing }}^{\mathrm{st}}(Z)
$$


restrict the same composition $I_{\lambda} \circ Q_{\rho}$ to the full subcategory $\mathrm{D}^{\mathrm{b}}(Z$-qcoh $) \subset$ $\mathrm{D}(Z$-qcoh $)$. According to the above, this restriction assigns to any bounded complex of quasicoherent sheaves $\mathcal{K}^{\bullet}$ on $Z$ the cone of a morphism $\mathcal{F}^{\bullet} \rightarrow \mathcal{K}^{\bullet}$ into it from its locally free left resolution $\mathcal{F}^{\bullet}$. Clearly, the functor

$$
\mathrm{D}^{\mathrm{b}}(Z \text {-qcoh }) \longrightarrow \mathrm{D}_{\text {Sing }}^{\text {st }}(Z)
$$

that we have constructed preserves those infinite direct sums that exist in $D^{\mathrm{b}}(Z$-qcoh) and annihilates the triangulated subcategory $\mathrm{D}^{\mathrm{b}}\left(Z\right.$-qcoh $\left.\mathrm{ff}_{\mathrm{If}}\right) \subset \mathrm{D}^{\mathrm{b}}(Z$-qcoh $)$. So we have the induced functor $\mathrm{D}_{\text {Sing }}^{\prime}(Z) \rightarrow \mathrm{D}_{\text {Sing }}^{\text {st }}(Z)$, and the first two assertions of the theorem are proven.

To prove the last assertion, we use the results of Section 2.8. Assume that $Z=X_{0}$ is the zero locus of a section $w \in \mathcal{L}(X)$ of a line bundle on $X$; as usual, $w: \mathcal{O}_{X} \rightarrow \mathcal{L}$ has to be an injective morphism of sheaves. Then by Theorem 2.8 and Lemma 2.8, the category $\mathrm{D}_{\text {Sing }}^{\prime}(Z)$ admits infinite direct sums and the image of the fully faithful functor $\mathrm{D}_{\text {Sing }}^{\mathrm{b}}\left(X_{0}\right) \rightarrow \mathrm{D}_{\text {Sing }}^{\prime}\left(X_{0}\right)$ is a set of compact generators in the target. Furthermore, it follows from the proof of Theorem 2.8 that any object of $\mathrm{D}_{\text {Sing }}^{\prime}\left(X_{0}\right)$ can be represented by a quasicoherent sheaf on $X_{0}$ and the direct sum of an infinite family of such objects is represented by the direct sums of such sheaves (see Remark 2.8). Thus the functor $D_{\text {Sing }}^{\prime}(Z) \rightarrow D_{\text {Sing }}^{\text {st }}(Z)$, being an infinite direct sum-preserving triangulated functor identifying triangulated subcategories of compact generators, is an equivalence of triangulated categories.

We keep the assumptions of Theorem 2.9 and the notation of the last paragraph of its proof; i.e., $X$ is a regular separated Noetherian scheme of finite Krull dimension with enough vector bundles and $X_{0} \subset X$ is the divisor of zeros of a locally nonzerodividing section $w \in \mathcal{L}(X)$. The closed embedding $X_{0} \rightarrow X$ is denoted by $i$.

\section{Corollary 2.9. The functor}

$$
\Lambda: \mathrm{D}^{\mathrm{co}}\left((X, \mathcal{L}, w)-\mathrm{qcoh}_{\mathrm{lf}}\right) \simeq \mathrm{D}^{\mathrm{co}}((X, \mathcal{L}, w)-\mathrm{qcoh}) \longrightarrow \mathrm{D}_{\text {Sing }}^{\text {st }}\left(X_{0}\right)
$$

assigning to a locally free (or just w-flat) quasicoherent matrix factorization $\mathcal{M}$ the acyclic complex of locally free (or quasicoherent) sheaves $i^{*} \mathcal{M}$ on $X_{0}$ is an equivalence of triangulated categories.

Proof. Given a $w$-flat matrix factorization $\mathcal{M}$, the complex of sheaves $i^{*} \mathcal{M}$ on $X_{0}$ is acyclic by [Polishchuk and Vaintrob 2011, Lemma 1.5]. Clearly, the assignment $\mathcal{M} \longmapsto i^{*} \mathcal{M}$ defines a triangulated functor $\mathrm{D}^{\mathrm{co}}\left((X, \mathcal{L}, w)\right.$-qcoh $\left.{ }_{w-\mathrm{fl}}\right) \rightarrow \mathrm{D}_{\text {Sing }}^{\text {st }}\left(X_{0}\right)$.

To prove that this functor is an equivalence of categories, it suffices to identify it, up to a shift, with the composition of the equivalences

$$
\mathrm{D}^{\mathrm{co}}\left((X, \mathcal{L}, w)-\mathrm{qcoh}_{\mathrm{lf}}\right) \longrightarrow \mathrm{D}_{\text {Sing }}^{\prime}\left(X_{0}\right) \longrightarrow \mathrm{D}_{\text {Sing }}^{\text {st }}\left(X_{0}\right) .
$$


Here one simply notices that for any $\mathcal{M} \in \mathrm{D}^{\mathrm{co}}\left((X, \mathcal{L}, w)\right.$-qcoh $\left.{ }_{\text {If }}\right)$ the complex $i^{*} \mathcal{M}$ is isomorphic in $\mathrm{D}_{\text {Sing }}^{\text {st }}\left(X_{0}\right)$ to its canonical truncation $\tau_{\leq 1} i^{*} \mathcal{M}$, and the latter complex is the cocone of the morphism into $\Sigma(\mathcal{M})$ from one of its left locally free resolutions. So the functor $\Lambda$ is identified with $\Sigma[-1]$.

2.10. Relative stable derived category. The goal of this section is to generalize the results of the previous one to the case of a singular Noetherian scheme $X$. The relative version of stable derived category, defined for a closed embedding of finite flat dimension $i: Z \rightarrow X$, is equivalent to the categories $\mathrm{D}_{\text {Sing }}^{\prime}\left(X_{0} / X\right)$ and $\mathrm{D}^{\mathrm{CO}}((X, \mathcal{L}, w)$-qcoh $)$ in the case of the Cartier divisor $Z=X_{0}$ corresponding to a locally nonzero-dividing section $w$ of a line bundle $\mathcal{L}$ on $X$.

Let $X$ be a separated Noetherian scheme of finite Krull dimension and $i: Z \rightarrow X$ be a closed embedding of schemes such that $i_{*} \mathcal{O}_{Z}$ has finite flat dimension as an $\mathcal{O}_{X}$-module. According to Section 1.9, there is a left derived inverse image functor $\mathbb{L} i^{*}: \mathrm{D}^{\mathrm{co}}(X$-qcoh $) \rightarrow \mathrm{D}^{\mathrm{co}}(Z$-qcoh $)$. This functor forms a commutative diagram with the similar functor $\mathbb{L} i^{*}: \mathrm{D}(X$-qcoh $) \rightarrow \mathrm{D}(Z$-qcoh $)$, and consequently, takes acyclic complexes in $\mathrm{D}^{\mathrm{co}}\left(X\right.$-qcoh) to acyclic complexes in $\mathrm{D}^{\mathrm{co}}(Z$-qcoh $)$.

Proposition 2.10. The following four triangulated categories are naturally equivalent:

(a) the full subcategory in $\mathrm{D}^{\mathrm{co}}(Z$-qcoh) consisting of all the objects annihilated by the direct image functor $i_{*}: \mathrm{D}^{\mathrm{co}}(Z$-qcoh $) \rightarrow \mathrm{D}^{\mathrm{co}}(X$-qcoh $)$;

(b) the quotient category of the homotopy category of complexes over $Z$-qcoh whose direct images are coacyclic complexes over $X$-qcoh by the thick subcategory of coacyclic complexes over $Z$-qcoh;

(c) the quotient category of $\mathrm{D}^{\mathrm{co}}(Z$-qcoh) by its minimal triangulated subcategory, containing the objects in $\mathbb{L} i^{*} \mathrm{D}^{\mathrm{co}}(X$-qcoh $)$ and closed under infinite direct sums;

(d) the quotient category of the full subcategory of acyclic complexes in $\mathrm{D}^{\mathrm{co}}(Z$-qcoh) by its minimal triangulated subcategory, containing the left derived inverse images of acyclic complexes in $\mathrm{D}^{\mathrm{co}}(X$-qcoh) and closed under infinite direct sums.

Proof. The equivalence of (a) and (b) is obvious. To show that the natural functor from the category (d) to the category (c) is an equivalence, notice that the minimal triangulated subcategory containing flat quasicoherent sheaves and closed under infinite direct sums together with the triangulated subcategory of acyclic complexes form a semiorthogonal decomposition of $\mathrm{D}^{\mathrm{co}}(X$-qcoh $)$, and similarly for $Z$ [Positselski 2012, Corollary A.4.7]. Since flat quasicoherent sheaves on $Z$ belong to the thick subcategory in $\mathrm{D}^{\mathrm{b}}(Z$-qcoh $) \subset \mathrm{D}^{\mathrm{co}}(Z$-qcoh $)$ generated by the inverse images of flat quasicoherent sheaves from $X$ (see the proof of Lemma 2.8), the assertion follows.

Finally, the functor $\mathbb{L} i^{*}$ preserves infinite direct sums and compactness of objects since its right adjoint functor $i_{*}$ preserves infinite direct sums. Hence the minimal 
triangulated subcategory in $\mathrm{D}^{\mathrm{co}}(Z$-qcoh $)$ containing $\mathbb{L} i^{*} \mathrm{D}^{\mathrm{co}}(X$-qcoh $)$ and closed under infinite direct sums is compactly generated by some objects which are compact in $\mathrm{D}^{\mathrm{co}}(Z$-qcoh). By Brown representability, the quotient category in (c) is equivalent to the right orthogonal complement to this triangulated subcategory, which is the kernel category in (a).

We call any of the equivalent triangulated categories in Proposition 2.10 the relative stable derived category of $Z$ over $X$ and denote it by $D_{\text {Sing }}^{\text {st }}(Z / X)$ (cf. [Becker 2014, Section 2]). In particular, defining the relative stable derived category by the construction (c), we have natural triangulated functors

$$
\mathrm{D}^{\mathrm{b}}(Z \text {-qcoh }) \longrightarrow \mathrm{D}^{\mathrm{co}}(Z \text {-qcoh }) \longrightarrow \mathrm{D}_{\text {Sing }}^{\mathrm{st}}(Z / X) .
$$

Clearly, the composition $\mathrm{D}^{\mathrm{b}}(Z$-qcoh $) \rightarrow \mathrm{D}_{\text {Sing }}^{\text {st }}(Z / X)$ factorizes through the relative singularity category $\mathrm{D}_{\text {Sing }}^{\prime}(Z / X)$, providing a natural functor $\mathrm{D}_{\text {Sing }}^{\prime}(Z / X) \rightarrow$ $\mathrm{D}_{\text {Sing }}^{\text {st }}(Z / X)$.

Lemma 2.10. The composition of triangulated functors

$$
\mathrm{D}_{\text {Sing }}^{\mathrm{b}}(Z / X) \longrightarrow \mathrm{D}_{\text {Sing }}^{\prime}(Z / X) \longrightarrow \mathrm{D}_{\text {Sing }}^{\text {st }}(Z / X)
$$

is fully faithful and its image forms a set of compact generators for the triangulated category $\mathrm{D}_{\text {Sing }}^{\text {st }}(Z / X)$.

Proof. By Proposition 1.5(d), the full triangulated subcategory $\mathrm{D}^{\mathrm{abs}}(Z$-coh) compactly generates the triangulated category $\mathrm{D}^{\mathrm{co}}(Z$-qcoh), and similarly this holds for $X$. In view of the construction (c) and the argument in the proof of Proposition 2.10, the assertion follows from [Neeman 1992, Theorem 2.1].

Now let $\mathcal{L}$ be a line bundle on $X$, let $w \in \mathcal{L}(X)$ be a locally nonzero-dividing section of $\mathcal{L}$, and let $i: X_{0} \rightarrow X$ be closed embedding of the zero locus of $w$. Defining the category $\mathrm{D}_{\text {Sing }}^{\text {st }}\left(X_{0} / X\right)$ by the construction $(\mathrm{d})$, let $\mathbb{L} \Lambda: \mathrm{D}^{\mathrm{co}}((X, \mathcal{L}, w)$-qcoh $) \rightarrow$ $\mathrm{D}_{\text {Sing }}^{\text {st }}\left(X_{0} / X\right)$ be the triangulated functor assigning to a $w$-flat quasicoherent matrix factorization $\mathcal{M}$ the acyclic complex $i^{*} \mathcal{M}$ over $X_{0}$-qcoh.

Since any bounded-below acyclic complex over $X_{0}$-qcoh is coacyclic, and any bounded-above complex belongs to the minimal triangulated subcategory in $\mathrm{D}^{\mathrm{co}}\left(X_{0}\right.$-qcoh $)$ generated by its terms and closed under infinite direct sums, the following diagram of triangulated functors is commutative (cf. Corollary 2.9):

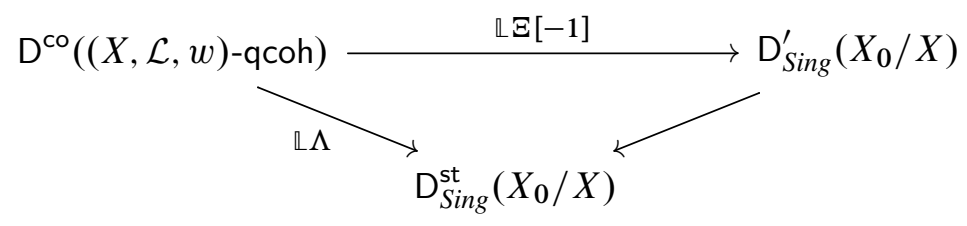


Theorem 2.10. For any locally nonzero-dividing section $w$ of a line bundle $\mathcal{L}$ on a separated Noetherian scheme $X$ of finite Krull dimension, all the three functors on the above diagram are equivalences of triangulated categories.

Proof. The functor $\mathbb{E}$ is an equivalence by Theorem 2.8. To show that the functor $\mathbb{L} \Lambda$ is an equivalence, let us check that it identifies compact generators. By Proposition $1.5(\mathrm{~d})$, the category $\mathrm{D}^{\mathrm{co}}((X, \mathcal{L}, w)$-qcoh) is compactly generated by its full triangulated subcategory $\mathrm{D}^{\text {abs }}((X, \mathcal{L}, w)$-coh), while according to Lemma 2.10 the category $\mathrm{D}_{\text {Sing }}^{\text {st }}\left(X_{0} / X\right)$ is compactly generated by its full triangulated subcategory $\mathrm{D}_{\text {Sing }}^{\mathrm{b}}\left(X_{0} / X\right)$. The restriction of the functor $\mathbb{L} \Lambda$ being an equivalence between these two subcategories (in view of commutativity of the diagram and) by Theorem 2.7, it follows that the functor $\mathbb{\Lambda} \Lambda$ itself is an equivalence, too.

Remark 2.10. Another proof of Theorem 2.10 can be obtained using the approach based on [Efimov 2013, Appendix A]. In the notation and assumptions of Remark 2.7, suppose that $C$ is an abelian category with exact functors of arbitrary infinite direct sums. Then so is the abelian category $\operatorname{MF}(\mathrm{C}, L, w)$; the full abelian subcategory $\mathrm{C}_{0} \subset \mathrm{C}$ is closed under infinite direct sums; and the triangulated functors $i_{*}, \mathbb{L} i^{*}, v^{n}, \mathbb{L} \xi^{n}, F^{n}, G^{n-}$ act between the coderived categories $\mathrm{D}^{\mathrm{co}}(\mathrm{C}), \mathrm{D}^{\mathrm{co}}\left(\mathrm{C}_{0}\right)$, and $\mathrm{D}^{\mathrm{CO}} M F(\mathrm{C}, L, w)$.

As in Remark 2.7, one proves that the functors $G^{n-}: \mathrm{D}^{\mathrm{co}}(\mathrm{C}) \rightarrow \mathrm{D}^{\mathrm{co}} M F(\mathrm{C}, L, w)$ and $v^{n}: \mathrm{D}^{\mathrm{co}}\left(\mathrm{C}_{0}\right) \rightarrow \mathrm{D}^{\mathrm{co}} M F(\mathrm{C}, L, w)$ are fully faithful and their images form a semiorthogonal decomposition of the coderived category $\mathrm{D}^{\mathrm{co}} M F(\mathrm{C}, L, w)$. By (the proof of) [loc. cit., Proposition A.3(3) and (4)], the totalization functor

$$
\mathrm{D}^{\mathrm{co}} \operatorname{MF}(\mathrm{C}, L, w) \longrightarrow \mathrm{D}^{\mathrm{co}}(\mathrm{C}, L, w)
$$

acting between the coderived category of the abelian category $\operatorname{MF}(\mathrm{C}, L, w)$ and the coderived category of matrix factorizations $\mathrm{D}^{\mathrm{CO}}(\mathrm{C}, L, w)$ (defined as in Section 1.3) is the Verdier localization by the minimal triangulated subcategory containing the objects $G^{n-}(B)$ and $G^{(n+1)-}(B)$ for all $B \in C$ and closed under infinite direct sums.

It follows that the composition of the functor $v^{n}: \mathrm{D}^{\mathrm{co}}\left(\mathrm{C}_{0}\right) \rightarrow \mathrm{D}^{\mathrm{co}} M F(\mathrm{C}, L, w)$ with the totalization functor $\mathrm{D}^{\mathrm{co}} M F(\mathrm{C}, L, w) \rightarrow \mathrm{D}^{\mathrm{co}}(\mathrm{C}, L, w)$ induces an equivalence of triangulated categories

$$
\mathrm{D}^{\mathrm{CO}}\left(\mathrm{C}_{0}\right) /\left\langle\mathbb{L} i^{*} \mathrm{D}^{\mathrm{CO}}(\mathrm{C})\right\rangle_{\oplus} \longrightarrow \mathrm{D}^{\mathrm{co}}(\mathrm{C}, L, w)
$$

between the quotient category of the coderived category $\mathrm{D}^{\mathrm{co}}\left(\mathrm{C}_{0}\right)$ by its minimal triangulated subcategory containing the image of the functor $\mathbb{L} i^{*}: \mathrm{D}^{\mathrm{CO}}(\mathrm{C}) \rightarrow \mathrm{D}^{\mathrm{CO}}\left(\mathrm{C}_{0}\right)$ and closed under infinite direct sums, and the coderived category of matrix factorizations. The composition of the functor $\mathbb{L} \xi^{n}: \mathrm{D}^{\mathrm{co}} M F(\mathrm{C}, L, w) \rightarrow \mathrm{D}^{\mathrm{co}}\left(\mathrm{C}_{0}\right)$ with the Verdier localization functor $\mathrm{D}^{\mathrm{CO}}\left(\mathrm{C}_{0}\right) \rightarrow \mathrm{D}^{\mathrm{CO}}\left(\mathrm{C}_{0}\right) /\left\langle\mathbb{L} i^{*} \mathrm{D}^{\mathrm{CO}}(\mathrm{C})\right\rangle_{\oplus}$ factorizes 
through the totalization functor, providing the inverse equivalence $\mathrm{D}^{\mathrm{co}}(\mathrm{C}, L, w) \rightarrow$ $\mathrm{D}^{\mathrm{CO}}\left(\mathrm{C}_{0}\right) /\left\langle\mathbb{L} i^{*} \mathrm{D}^{\mathrm{co}}(\mathrm{C})\right\rangle_{\oplus}$.

Returning to quasicoherent matrix factorizations of a global section $w \in \mathcal{L}(X)$ of a line bundle $\mathcal{L}$ on a separated Noetherian scheme $X$ with the zero locus $X_{0} \subset X$, we obtain direct constructions of two mutually inverse triangulated equivalences between the coderived category $\mathrm{D}^{\mathrm{co}}((X, \mathcal{L}, w)$-qcoh) and the relative stable derived category $\mathrm{D}_{\text {Sing }}^{\text {st }}\left(X_{0} / X\right)$ as defined in part (c) of Proposition 2.10.

\section{Supports, pull-backs, and push-forwards}

3.1. Supports. This section paves the ground for the results about preservation of finite rank or coherence by the push-forwards of matrix factorizations with proper supports, which will be proven in Sections 3.5-3.6.

Let $X$ be a separated Noetherian scheme and $T \subset X$ be a Zariski closed subset. Denote by $X$-coh ${ }_{T}$ the abelian category of coherent sheaves on $X$ with set-theoretic support in $T$; and we will use similar notation for quasicoherent sheaves.

It is a well-known fact (essentially, a reformulation of the Artin-Rees lemma) that the embedding of abelian categories $X$-qcoh ${ }_{T} \rightarrow X$-qcoh takes injectives to injectives. It follows that the functor $\mathrm{D}^{\mathrm{b}}\left(X-\mathrm{coh}_{T}\right) \rightarrow \mathrm{D}^{\mathrm{b}}(X$-coh $)$ is fully faithful. Clearly, its image is a thick subcategory and the corresponding quotient category can be naturally identified with $\mathrm{D}^{\mathrm{b}}(U$-coh), where $U=X \backslash T$ (cf. Section 1.10).

Assume additionally that $X$ has enough vector bundles. Let $\operatorname{Perf}_{T}(X) \subset \operatorname{Perf}(X)$ denote the full subcategory of perfect complexes with the cohomology sheaves settheoretically supported in $T$. By the above result, $\operatorname{Perf}_{T}(X)$ can be considered as a thick subcategory in $\mathrm{D}^{\mathrm{b}}\left(X-\operatorname{coh}_{T}\right)$. According to [Orlov 2011, Lemma 2.6], the functor $\mathrm{D}^{\mathrm{b}}(X$-coh $T) / \operatorname{Perf}_{T}(X) \rightarrow \mathrm{D}_{\text {Sing }}^{\mathrm{b}}(X)$ induced by the embedding $\mathrm{D}^{\mathrm{b}}\left(X\right.$-coh $\left._{T}\right) \rightarrow$ $\mathrm{D}^{\mathrm{b}}(X$-coh) is fully faithful. We denote the source (or the image) category of this functor by $\mathrm{D}_{\text {Sing }}^{\mathrm{b}}(X, T)$.

By [Chen 2010, Theorem 1.3], the restriction functor $D_{\text {Sing }}^{\mathrm{b}}(X) \rightarrow \mathrm{D}_{\text {Sing }}^{\mathrm{b}}(U)$ is the Verdier localization functor by the triangulated subcategory $\mathrm{D}_{\text {Sing }}^{\mathrm{b}}(X, T)$. In particular, the kernel of the restriction functor coincides with the thick envelope of (i.e., the minimal thick subcategory containing) $\mathrm{D}_{\text {Sing }}^{\mathrm{b}}(X, T)$ in $\mathrm{D}_{\text {Sing }}^{\mathrm{b}}(X)$.

Now we are going to establish the similar results for the triangulated categories of relative singularities. Let $i: Z \rightarrow X$ be a closed subscheme such that $i_{*} \mathcal{O}_{Z} \in \operatorname{Perf}(X)$, and let $\operatorname{Perf}(Z / X)=\mathrm{D}^{\mathrm{b}}\left(\mathrm{E}_{Z / X}\right)$ (see Remark 2.1) denote the thick subcategory in $\mathrm{D}^{\mathrm{b}}\left(Z\right.$-coh) generated by $\mathbb{L} i^{*} \mathrm{D}^{\mathrm{b}}(X$-coh $)$. Let $T \subset Z$ be a Zariski closed subset; put $U=X \backslash T$ and $V=Z \backslash T$. We denote by $\operatorname{Perf}_{T}(Z / X)$ the full subcategory of all objects of $\operatorname{Perf}(Z / X)$ with the cohomology sheaves set-theoretically supported in $T$. Consider it as a thick subcategory in $\mathrm{D}^{\mathrm{b}}(Z$-coh $T)$, and denote by $\mathrm{D}_{\text {Sing }}^{\mathrm{b}}(Z / X, T)$ the quotient category $\mathrm{D}^{\mathrm{b}}(Z$-coh $T) / \operatorname{Perf}_{T}(Z / X)$. 
Lemma 3.1. (a) The functor $\mathrm{D}_{\text {Sing }}^{\mathrm{b}}(Z / X, T) \rightarrow \mathrm{D}_{\text {Sing }}^{\mathrm{b}}(Z / X)$ induced by the embedding $\mathrm{D}^{\mathrm{b}}\left(Z\right.$ - $\left.\operatorname{coh}_{T}\right) \rightarrow \mathrm{D}^{\mathrm{b}}(Z$-coh $)$ is fully faithful.

(b) The restriction functor $\mathrm{D}_{\text {Sing }}^{\mathrm{b}}(Z / X) \rightarrow \mathrm{D}_{\text {Sing }}^{\mathrm{b}}(V / U)$ is the Verdier localization functor by the triangulated subcategory $\mathrm{D}_{\text {Sing }}^{\mathrm{b}}(Z / X, T)$. In particular, the kernel of the restriction functor coincides with the thick envelope of $\mathrm{D}_{\text {Sing }}^{\mathrm{b}}(Z / X, T)$ in $\mathrm{D}_{\text {Sing }}^{\mathrm{b}}(Z / X)$.

Proof. The proof of (a) is similar to that of [Orlov 2011, Lemma 2.6]. One only needs to notice that the tensor product of an object of $\operatorname{Perf}(Z / X)$ with an object of $\operatorname{Perf}(Z)$ belongs to $\operatorname{Perf}(Z / X)$. This follows from the fact that $\operatorname{Perf}(Z)$ as a thick subcategory in $\mathrm{D}^{\mathrm{b}}(Z$-coh $)$ is generated by the restrictions of vector bundles from $X$ (see Section 2.1). Part (b) is true since the thick subcategory $\operatorname{Perf}(V / U) \subset \mathrm{D}^{\mathrm{b}}(V$-coh $)$ is generated by the image of the restriction functor $\operatorname{Perf}(Z / X) \rightarrow \operatorname{Perf}(V / U)$, which is true because any coherent sheaf on $U$ can be extended to a coherent sheaf on $X$.

Let $\mathcal{L}$ be a line bundle over $X$ and $w \in \mathcal{L}(X)$ be a section; set $X_{0}=\{w=0\} \subset X$. The definitions of the set-theoretic and category-theoretic supports $\operatorname{Supp} \mathcal{M}$ and supp $\mathcal{M}$ of a coherent matrix factorization $\mathcal{M} \in(X, \mathcal{L}, w)$-coh were given (in a greater generality of coherent CDG-modules) in Section 1.10.

Given a locally free matrix factorization of finite rank $\mathcal{M} \in(X, \mathcal{L}, w)$-coh ${ }_{\mathrm{If}}$, define the (category-theoretic) support $\operatorname{supp} \mathcal{M} \subset X$ as the minimal closed subset $T \subset X$ such that the restriction $\left.\mathcal{M}\right|_{U}$ of $\mathcal{M}$ to the open subscheme $U=X \backslash T$

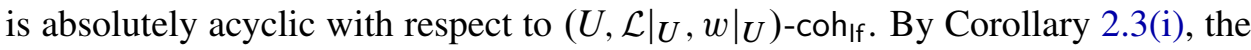
definitions of category-theoretic supports of coherent matrix factorizations and of locally free matrix factorizations of finite rank agree when they are both applicable.

Equivalently, for a locally free matrix factorization $\mathcal{M}$ of finite rank over $X$, the open subscheme $X \backslash \operatorname{supp} \mathcal{M}$ is the union of all affine open subschemes $U \subset X$ such that the matrix factorization $\left.\mathcal{M}\right|_{U}$ is contractible (see Remark 1.3). For any coherent matrix factorization $\mathcal{M}$, one has supp $\mathcal{M} \subset X_{0}$ since any matrix factorization of an invertible potential is contractible (cf. [Polishchuk and Vaintrob 2011, Section 5]).

Let $T \subset X$ be a closed subset. Denote by $\mathrm{D}_{T}^{\mathrm{abs}}((X, \mathcal{L}, w)$-coh If $)$ (respectively $\mathrm{D}_{T}^{\text {abs }}((X, \mathcal{L}, w)$-coh $\left.)\right)$ the quotient category of the homotopy category of locally free matrix factorizations of finite rank (resp. coherent matrix factorizations) supported category-theoretically inside $T$ by the thick subcategory of matrix factorizations absolutely acyclic with respect to $(X, \mathcal{L}, w)$-coh If $_{\text {(resp. }}(X, \mathcal{L}, w)$-coh). Clearly, the functors $\mathrm{D}_{T}^{\text {abs }}((X, \mathcal{L}, w)$-coh If $) \rightarrow \mathrm{D}^{\text {abs }}((X, \mathcal{L}, w)$-coh If $)$ and $\mathrm{D}_{T}^{\text {abs }}((X, \mathcal{L}, w)$-coh $) \rightarrow$ $\mathrm{D}^{\text {abs }}((X, \mathcal{L}, w)$-coh $)$ are fully faithful [loc. cit.].

By the definition, the thick subcategories

$$
\begin{gathered}
\mathrm{D}_{T}^{\text {abs }}\left((X, \mathcal{L}, w)-\text { coh }_{\text {If }}\right) \subset \mathrm{D}^{\text {abs }}\left((X, \mathcal{L}, w)-\text { coh }_{\text {If }}\right), \\
\mathrm{D}_{T}^{\text {abs }}((X, \mathcal{L}, w) \text {-coh }) \subset \mathrm{D}^{\text {abs }}((X, \mathcal{L}, w)-c o h)
\end{gathered}
$$


only depend on the intersection $X_{0} \cap T$ (rather than the whole of $T$ ). Equivalently, they can be defined as the full subcategories of objects annihilated by the restriction functors $\mathrm{D}^{\mathrm{abs}}\left((X, \mathcal{L}, w)\right.$-coh $\left.{ }_{\text {If }}\right) \rightarrow \mathrm{D}^{\text {abs }}\left(\left(U,\left.\mathcal{L}\right|_{U},\left.w\right|_{U}\right)\right.$-coh $\left.{ }_{\text {If }}\right)$ and $\mathrm{D}^{\text {abs }}((X, \mathcal{L}, w)$-coh $) \rightarrow \mathrm{D}^{\text {abs }}\left(\left(U,\left.\mathcal{L}\right|_{U},\left.w\right|_{U}\right)\right.$-coh $)$, where $U=X \backslash T$.

As in Section 1.10 , we denote by $\mathrm{D}^{\text {abs }}\left((X, \mathcal{L}, w)-\operatorname{coh}_{T}\right)$ the absolute derived category of coherent matrix factorizations with set-theoretic support in $T$. The functor $\mathrm{D}^{\text {abs }}\left((X, \mathcal{L}, w)-\operatorname{coh}_{T}\right) \rightarrow \mathrm{D}^{\text {abs }}((X, \mathcal{L}, w)$-coh) is fully faithful by Proposition 1.10(d). By Corollary 1.10(b), the full subcategory

$$
\mathrm{D}_{T}^{\mathrm{abs}}((X, \mathcal{L}, w) \text {-coh }) \subset \mathrm{D}^{\mathrm{abs}}((X, \mathcal{L}, w) \text {-coh })
$$

is the thick envelope of the full subcategory $\mathrm{D}^{\mathrm{abs}}\left((X, \mathcal{L}, w)-\operatorname{coh}_{T}\right)$.

Now assume that $w: \mathcal{O}_{X} \rightarrow \mathcal{L}$ is an injective morphism of sheaves.

Proposition 3.1. (a) The equivalence of categories

$$
\mathrm{D}^{\mathrm{abs}}((X, \mathcal{L}, w)-\mathrm{coh}) \simeq \mathrm{D}_{\text {Sing }}^{\mathrm{b}}\left(X_{0} / X\right)
$$

identifies the triangulated subcategory $\mathrm{D}^{\mathrm{abs}}\left((X, \mathcal{L}, w)-\operatorname{coh}_{T}\right)$ with the triangulated subcategory $\mathrm{D}_{\text {Sing }}^{\mathrm{b}}\left(X_{0} / X, X_{0} \cap T\right)$. In particular, the former triangulated subcategory only depends on the intersection $X_{0} \cap T$.

(b) The full preimage of the thick envelope of the triangulated subcategory

$$
\mathrm{D}_{\text {Sing }}^{\mathrm{b}}\left(X_{0}, X_{0} \cap T\right) \subset \mathrm{D}_{\text {Sing }}^{\mathrm{b}}\left(X_{0}\right)
$$

under the fully faithful functor $\Sigma: \mathrm{D}^{\mathrm{abs}}\left((X, \mathcal{L}, w)\right.$-coh $\left.\mathrm{ch}_{\mathrm{ff}}\right) \rightarrow \mathrm{D}_{\text {Sing }}^{\mathrm{b}}\left(X_{0}\right)$ coincides with the triangulated subcategory $\mathrm{D}_{T}^{\text {abs }}\left((X, \mathcal{L}, w)-\mathrm{coh}_{\mathrm{If}}\right)$.

Proof. Part (b) follows from the fact that the thick envelope of $D_{\text {Sing }}^{\mathrm{b}}\left(X_{0}, X_{0} \cap T\right)$ is the kernel of the restriction functor $D_{\text {Sing }}^{\mathrm{b}}\left(X_{0}\right) \rightarrow \mathrm{D}_{\text {Sing }}^{\mathrm{b}}\left(X_{0} \backslash T\right)$, the similar fact for $\mathrm{D}_{T}^{\text {abs }}\left((X, \mathcal{L}, w)\right.$-coh $\left.{ }_{\text {If }}\right)$, and the compatibility of the functors $\Sigma$ with the restrictions to open subschemes, together with their full-and-faithfulness.

To prove part (a), notice first that the functor $\Upsilon$ obviously takes $D_{\text {Sing }}^{\mathrm{b}}\left(X_{0} / X\right.$, $\left.X_{0} \cap T\right)$ into $\mathrm{D}^{\text {abs }}\left((X, \mathcal{L}, w)-\operatorname{coh}_{T}\right)$. Let us check that the functor $\mathbb{E} \Xi$ takes $\mathrm{D}^{\text {abs }}((X, \mathcal{L}, w)$-coh $T)$ into $\mathrm{D}_{\text {Sing }}^{\mathrm{b}}\left(X_{0} / X, X_{0} \cap T\right)$. Let $\mathcal{M}$ be a coherent matrix factorization supported set-theoretically in $T$. Present $\mathcal{M}$ as the cokernel of an injective morphism of $w$-flat coherent matrix factorizations $\mathcal{K} \rightarrow \mathcal{N}$. Since the functor $\mathbb{L} \Xi$ is triangulated, the object $\mathbb{\Xi}(\mathcal{M}) \in \mathrm{D}_{\text {Sing }}^{\mathrm{b}}\left(X_{0} / X\right)$ is isomorphic to the cone of the morphism $\Xi(\mathcal{K}) \rightarrow \Xi(\mathcal{N})$ (cf. Lemma 3.6). The morphism $\Xi(\mathcal{K}) \rightarrow \Xi(\mathcal{N})$ of coherent sheaves on $X_{0}$ is an isomorphism outside $T$, so its kernel and cokernel are supported in $X_{0} \cap T$. Thus the cone is quasi-isomorphic to a two-term complex of coherent sheaves on $X_{0}$ with the terms supported set-theoretically in $X_{0} \cap T$. 
3.2. Locality of local freeness. The aim of this section is to show that the property of an object of $\mathrm{D}^{\text {abs }}\left((X, \mathcal{L}, w)\right.$-qcoh $\left.{ }_{\mathrm{fl}}\right)$ or $\mathrm{D}^{\text {abs }}((X, \mathcal{L}, w)$-coh) to be a direct summand of an object from $\mathrm{D}^{\text {abs }}\left((X, \mathcal{L}, w)\right.$-coh $\left.{ }_{\text {lf }}\right)$ is local in a separated Noetherian scheme $X$ with a dualizing complex and enough vector bundles, assuming that the potential $w \in \mathcal{L}(X)$ is not locally zero-dividing.

Let $Z$ be a Noetherian scheme of finite Krull dimension with enough vector bundles. Recall that the natural functor $\mathrm{D}_{\text {Sing }}^{\mathrm{b}}(Z) \rightarrow \mathrm{D}_{\text {Sing }}^{\prime}(Z)$ is fully faithful [Orlov 2004, Proposition 1.13] (cf. Section 2.8).

Proposition 3.2. Let $Z=U \cup V$ be a covering by two open subschemes. Then any object of $\mathrm{D}_{\text {Sing }}^{\prime}(Z)$ whose restrictions to $U$ and $V$ belong to the full subcategories $\mathrm{D}_{\text {Sing }}^{\mathrm{b}}(U) \subset \mathrm{D}_{\text {Sing }}^{\prime}(U)$ and $\mathrm{D}_{\text {Sing }}^{\mathrm{b}}(V) \subset \mathrm{D}_{\text {Sing }}^{\prime}(V)$, respectively, is a direct summand of an object belonging to the full subcategory $\mathrm{D}_{\text {Sing }}^{\mathrm{b}}(Z) \subset \mathrm{D}_{\text {Sing }}^{\prime}(Z)$.

Proof. Consider the bounded derived category of quasicoherent sheaves $\mathrm{D}^{\mathrm{b}}(Z$-qcoh) on $Z$ and two full triangulated subcategories $\mathrm{D}^{\mathrm{b}}(Z$-coh $)$ and $\mathrm{D}^{\mathrm{b}}\left(Z\right.$-qcoh $\left.\mathrm{fl}_{\mathrm{fl}}\right)$ in it. Clearly, the intersection $D^{\mathrm{b}}(Z$-coh $) \cap D^{\mathrm{b}}\left(Z\right.$-qcoh $\left.{ }_{\mathrm{fl}}\right)$ coincides with the full subcategory of perfect complexes $\operatorname{Perf}(Z)=\mathrm{D}^{\mathrm{b}}(Z$-coh lf $) \subset \mathrm{D}^{\mathrm{b}}(Z$-qcoh $)$.

Lemma 3.2. Any morphism from an object of the full subcategory $D^{b}\left(Z-q c o h_{f l}\right)$ into an object of the full subcategory $\mathrm{D}^{\mathrm{b}}(Z$-coh $) \subset \mathrm{D}^{\mathrm{b}}(Z$-qcoh $)$ factorizes through an object belonging to $\mathrm{D}^{\mathrm{b}}(Z$-coh $\mathrm{lf})$.

Proof. See the proof of [Orlov 2004, Proposition 1.13].

It follows from Lemma 3.2 ( by the way of the octahedron axiom) that any object $\mathcal{K}^{\bullet}$ of the full triangulated subcategory $\mathrm{D}^{\mathrm{b}}(Z \text {-qcoh })_{\mathrm{fl}-\mathrm{c}}$ generated by $\mathrm{D}^{\mathrm{b}}\left(Z\right.$-qcoh $\left.\mathrm{fl}_{\mathrm{fl}}\right)$ and $D^{\mathrm{b}}(Z$-coh $)$ in $\mathrm{D}^{\mathrm{b}}(Z$-qcoh $)$ can be included in a distinguished triangle

$$
\mathcal{F}^{\bullet} \longrightarrow \mathcal{K}^{\bullet} \longrightarrow \mathcal{M}^{\bullet} \longrightarrow \mathcal{F}^{\bullet}[1],
$$

with $\mathcal{F}^{\bullet} \in \mathrm{D}^{\mathrm{b}}(Z$-qcoh fl $)$ and $\mathcal{M}^{\bullet} \in \mathrm{D}^{\mathrm{b}}(Z$-coh $)$. Besides, the natural functor $\mathrm{D}^{\mathrm{b}}(Z$-qcoh $\mathrm{fl}) / \mathrm{D}^{\mathrm{b}}(Z$-coh ff $) \rightarrow \mathrm{D}^{\mathrm{b}}(Z$-qcoh $) / \mathrm{D}^{\mathrm{b}}(Z$-coh $)$ is fully faithful.

To prove Proposition 3.2, one has to show that any object $\mathcal{K}^{\bullet} \in \mathrm{D}^{\mathrm{b}}(Z$-qcoh $)$ whose restrictions to $U$ and $V$ belong to the subcategories $\mathrm{D}^{\mathrm{b}}(U-\mathrm{qcoh})_{\mathrm{fl-c}}$ and $\mathrm{D}^{\mathrm{b}}\left(V-\mathrm{qcoh} \mathrm{fl-c}_{\mathrm{c}}\right)$, respectively, is a direct summand of an object from $\mathrm{D}^{\mathrm{b}}(Z \text {-qcoh })_{\mathrm{fl}-\mathrm{c}} \subset$ $\mathrm{D}^{\mathrm{b}}(Z$-qcoh $)$. According to the above, there exist two objects $\mathcal{F}_{U}^{\bullet} \in \mathrm{D}^{\mathrm{b}}\left(U\right.$-qcoh $\left.\mathrm{fl}_{\mathrm{fl}}\right)$ and $\mathcal{F}_{V}^{\bullet} \in \mathrm{D}^{\mathrm{b}}(V$-qcoh fl $)$ and two morphisms $\left.\mathcal{F}_{U}^{\bullet} \rightarrow \mathcal{K}^{\bullet}\right|_{U}$ and $\left.\mathcal{F}_{V}^{\bullet} \rightarrow \mathcal{K}^{\bullet}\right|_{V}$ whose cones belong to $\mathrm{D}^{\mathrm{b}}(U$-coh $)$ and $\mathrm{D}^{\mathrm{b}}(V$-coh $)$, respectively.

Set $W=U \cap V \subset Z$; then the restrictions of $\mathcal{F}_{U}^{\bullet}$ and $\mathcal{F}_{V}^{\bullet}$ to $W$ are isomorphic in $\mathrm{D}^{\mathrm{b}}(W-\mathrm{q} c o h) / \mathrm{D}^{\mathrm{b}}(W-c o h)$, and consequently, in $\mathrm{D}^{\mathrm{b}}\left(W-\mathrm{qcoh}_{\mathrm{fl}}\right) / \mathrm{D}^{\mathrm{b}}\left(W-\operatorname{coh}_{\mathrm{If}}\right)$, too. Notice that the category $\operatorname{Perf}(W)=\mathrm{D}^{\mathrm{b}}\left(W-\operatorname{coh}_{\mathrm{If}}\right)$ is idempotent complete, and therefore, a thick subcategory in $\mathrm{D}^{\mathrm{b}}\left(W-\mathrm{q} c o \mathrm{~h}_{\mathrm{fl}}\right)$. It follows that there exists a finite complex of flat quasicoherent sheaves $\mathcal{F}_{W}^{\bullet}$ on $W$ together with two morphisms 
$\left.\mathcal{F}_{U}^{\bullet}\right|_{W} \rightarrow \mathcal{F}_{W}^{\bullet}$ and $\left.\mathcal{F}_{V}^{\bullet}\right|_{W} \rightarrow \mathcal{F}_{W}^{\bullet}$ whose cones are perfect complexes. Denote the cocones of these morphisms by $\mathcal{G}_{W}^{\bullet}$ and $\mathcal{H}_{W}^{\bullet}$.

For any object $A$ of a triangulated category $\mathrm{D}$, let us denote by ' $A$ the object $A \oplus A[1]$. For any triangulated subcategory $\mathrm{C} \subset \mathrm{D}$, whenever an object $A \in \mathrm{D}$ is a direct summand of an object from C, the object ' $A$ belongs to C, as $A \oplus B \in \mathrm{C}$ implies $A \oplus A[1] \in \mathrm{C}$ in view of the distinguished triangle $A \oplus B \rightarrow A \oplus B \rightarrow$ $A \oplus A[1] \rightarrow A[1] \oplus B[1]$ [Thomason 1997, Theorem 2.1].

By the Thomason-Trobaugh theorem [1990, Section 5], the objects ' $\mathcal{G}_{W}^{\bullet}$ and $^{\prime} \mathcal{H}_{W}^{\bullet}$ can be extended to perfect complexes on $U$ and $V$, respectively. Moreover, these extensions $\mathcal{G}_{U}^{\bullet} \in \mathrm{D}^{\mathrm{b}}(U$-coh If $)$ and $\mathcal{H}_{V}^{\bullet} \in \mathrm{D}^{\mathrm{b}}\left(V\right.$-coh $\left.{ }_{\text {If }}\right)$ can be chosen in such a way that the morphisms $\left.{ }^{\prime} \mathcal{G}_{W}^{\bullet} \rightarrow{ }^{\prime} \mathcal{F}_{U}^{\bullet}\right|_{W}$ and $\left.{ }^{\prime} \mathcal{H}_{W}^{\bullet} \rightarrow{ }^{\prime} \mathcal{F}_{V}^{\bullet}\right|_{W}$ would be extendable to morphisms $\mathcal{G}_{U}^{\bullet} \rightarrow^{\prime} \mathcal{F}_{U}^{\bullet}$ and $\mathcal{H}_{V}^{\bullet} \rightarrow^{\prime} \mathcal{F}_{V}^{\bullet}$ [Neeman 1996, Theorem 2.1(4) and (5)].

Furthermore, the objects ' $\mathcal{G}_{U}^{\bullet}$ and ${ }^{\prime} \mathcal{H}_{V}^{\bullet}$ can be extended to perfect complexes $\mathcal{G}^{\bullet}$ and $\mathcal{H}^{\bullet}$ on the whole scheme $Z$ so that the compositions of morphisms

$$
\left.{ }^{\prime} \mathcal{G}_{U}^{\bullet} \longrightarrow{ }^{\prime \prime} \mathcal{F}_{U}^{\bullet} \longrightarrow{ }^{\prime \prime} \mathcal{K} \bullet\right|_{U} \quad \text { and }\left.\quad{ }^{\prime} \mathcal{H}_{V}^{\bullet} \longrightarrow{ }^{\prime \prime} \mathcal{F}_{V}^{\bullet} \longrightarrow{ }^{\prime \prime} \mathcal{K}^{\bullet}\right|_{V}
$$

would be extendable to morphisms $\mathcal{G}^{\bullet} \rightarrow^{\prime \prime} \mathcal{K}^{\bullet}$ and $\mathcal{H}^{\bullet} \rightarrow{ }^{\prime \prime} \mathcal{K} \bullet$. Denote by $\mathcal{K}_{(1)}^{\bullet}$ a cone of the morphism $\mathcal{G}^{\bullet} \oplus \mathcal{H}^{\bullet} \rightarrow^{\prime \prime} \mathcal{K}^{\bullet}$, by $\mathcal{F}_{U,(1)}^{\bullet}$ a cone of the morphism ${ }^{\prime} \mathcal{G}_{U}^{\bullet} \rightarrow{ }^{\prime \prime} \mathcal{F}_{U}^{\bullet}$, and by $\mathcal{F}_{V,(1)}^{\bullet}$ a cone of the morphism ' $\mathcal{H}_{V}^{\bullet} \rightarrow{ }^{\prime \prime} \mathcal{F}_{V}^{\bullet}$. We have come back to the original situation with an object $\mathcal{K}_{(1)}^{\bullet} \in \mathrm{D}^{\mathrm{b}}(Z$-qcoh $)$, two objects $\mathcal{F}_{U,(1)}^{\bullet} \in \mathrm{D}^{\mathrm{b}}\left(U\right.$-qcoh $\left._{\mathrm{fl}}\right)$ and $\mathcal{F}_{V,(1)}^{\bullet} \in \mathrm{D}^{\mathrm{b}}\left(V\right.$-qcoh $\left.\mathrm{fl}_{\mathrm{fl}}\right)$, and two morphisms

$$
\left.\mathcal{F}_{U,(1)}^{\bullet} \longrightarrow \mathcal{K}_{(1)}^{\bullet}\right|_{U} \quad \text { and }\left.\quad \mathcal{F}_{V,(1)}^{\bullet} \longrightarrow \mathcal{K}_{(1)}^{\bullet}\right|_{V}
$$

whose cones belong to $\mathrm{D}^{\mathrm{b}}(U-\mathrm{coh})$ and $\mathrm{D}^{\mathrm{b}}(V$-coh $)$, respectively. In addition, the objects $\mathcal{F}_{U,(1)}^{\bullet} \mid W$ and $\mathcal{F}_{V,(1)}^{\bullet} \mid W$ are now isomorphic in $\mathrm{D}^{\mathrm{b}}\left(W\right.$-qcoh $\left._{\mathrm{fl}}\right)$.

The construction does not guarantee commutativity of the diagram formed by the isomorphism

$$
\left.\mathcal{F}_{U,(1)}^{\bullet}\right|_{W}=\left.\mathcal{F}_{W,(1)}^{\bullet} \simeq \mathcal{F}_{V,(1)}^{\bullet}\right|_{W}
$$

and the restrictions of the morphisms $\mathcal{F}_{U,(1)}^{\bullet} \rightarrow \mathcal{K}_{(1)}^{\bullet}$ and $\mathcal{F}_{V,(1)}^{\bullet} \rightarrow \mathcal{K}_{(1)}^{\bullet}$ to $W$. However, the original choice of the morphisms

$$
\left.\mathcal{F}_{U}^{\bullet}\right|_{W} \longrightarrow \mathcal{F}_{W}^{\bullet} \quad \text { and }\left.\quad \mathcal{F}_{V}^{\bullet}\right|_{W} \longrightarrow \mathcal{F}_{W}^{\bullet}
$$

makes this diagram commute in the quotient category $\mathrm{D}^{b}(W$-qcoh $) / \mathrm{D}^{\mathrm{b}}(W$-coh $)$. Hence the difference of two morphisms $\mathcal{F}_{W,(1)}^{\bullet} \rightrightarrows \mathcal{K}_{(1)}^{\bullet} \mid W$ factorizes through a bounded complex of coherent sheaves on $W$, and consequently (according to Lemma 3.2) also through a perfect complex on $W$. Denote the latter by $\mathcal{E}^{\bullet} \in$ $\mathrm{D}^{\mathrm{b}}\left(W-\mathrm{coh}_{\mathrm{If}}\right)$. 
Now let $j: U \rightarrow Z, k: V \rightarrow Z$, and $h: W \rightarrow Z$ denote the natural open embeddings. Consider the square diagram formed by the morphisms

$$
\begin{gathered}
\left.\mathbb{R} j_{*} \mathcal{F}_{U,(1)}^{\bullet} \oplus \mathbb{R} k_{*} \mathcal{F}_{V,(1)}^{\bullet} \longrightarrow \mathbb{R} h_{*} \mathcal{F}_{U,(1)}^{\bullet}\right|_{W}, \\
\left.\left.\left.\mathbb{R} j_{*} \mathcal{K}_{(1)}^{\bullet}\right|_{U} \oplus \mathbb{R} k_{*} \mathcal{K}_{(1)}^{\bullet}\right|_{V} \longrightarrow \mathbb{R} h_{*} \mathcal{K}_{(1)}^{\bullet}\right|_{W}
\end{gathered}
$$

According to the above, this diagram is not necessarily commutative; but it can be made commutative by adding the new direct summand $\mathbb{R} h_{*} \mathcal{E}^{\bullet}$ to the term $\left.\left.\mathbb{R} j_{*} \mathcal{K}_{(1)}^{\bullet}\right|_{U} \oplus \mathbb{R} k_{*} \mathcal{K}_{(1)}^{\bullet}\right|_{V}$ with the morphism $\left.\mathbb{R} h_{*} \mathcal{E}^{\bullet} \rightarrow \mathbb{R} h_{*} \mathcal{K}_{(1)}^{\bullet}\right|_{W}$ induced by the morphism $\mathcal{E}^{\bullet} \rightarrow \mathcal{K}_{(1)}^{\bullet} \mid W$ and the morphism $\mathbb{R} j_{*} \mathcal{F}_{U,(1)}^{\bullet} \oplus \mathbb{R} k_{*} \mathcal{F}_{V,(1)}^{\bullet}$ equal to zero on the first direct summand and induced by the morphism $\mathcal{F}_{V,(1)}^{\bullet} \mid W \underset{W}{\simeq \mathcal{F}_{W,(1)}^{\bullet}} \rightarrow \mathcal{E}^{\bullet}$ on the second one.

Let $\mathcal{F}^{\bullet}$ denote a cocone of the morphism

$$
\left.\mathbb{R} j_{*} \mathcal{F}_{U,(1)}^{\bullet} \oplus \mathbb{R} k_{*} \mathcal{F}_{V,(1)}^{\bullet} \longrightarrow \mathbb{R} h_{*} \mathcal{F}_{U,(1)}^{\bullet}\right|_{W}
$$

and $\mathcal{L}^{\bullet}$ denote a cocone of the morphism

$$
\left.\left.\left.\mathbb{R} j_{*} \mathcal{K}_{(1)}^{\bullet}\right|_{U} \oplus \mathbb{R} k_{*} \mathcal{K}_{(1)}^{\bullet}\right|_{V} \oplus \mathbb{R} h_{*} \mathcal{E}^{\bullet} \longrightarrow \mathbb{R} h_{*} \mathcal{K}_{(1)}^{\bullet}\right|_{W}
$$

Then the commutative square can be extended to a morphism of distinguished triangles, so we obtain a morphism $\mathcal{F}^{\bullet} \rightarrow \mathcal{L}^{\bullet}$. Since $\mathcal{K}_{(1)}^{\bullet}$ is a cocone of the morphism

$$
\left.\left.\left.\mathbb{R} j_{*} \mathcal{K}_{(1)}^{\bullet}\right|_{U} \oplus \mathbb{R} k_{*} \mathcal{K}_{(1)}^{\bullet}\right|_{V} \longrightarrow \mathbb{R} h_{*} \mathcal{K}_{(1)}^{\bullet}\right|_{W},
$$

there is also a distinguished triangle $\mathcal{K}_{(1)}^{\bullet} \rightarrow \mathcal{L}^{\bullet} \rightarrow \mathbb{R} h_{*} \mathcal{E} \rightarrow \mathcal{K}_{(1)}^{\bullet}[1]$.

Notice that the complexes $\mathcal{F}^{\bullet}$ and $\mathbb{R} h_{*} \mathcal{E}^{\bullet}$ belong to $\mathrm{D}^{\mathrm{b}}\left(Z\right.$-qcoh $\left.{ }_{\mathrm{fl}}\right)$ (since the class of bounded complexes of flat quasicoherent sheaves is preserved by the derived direct images with respect to flat morphisms of Noetherian schemes; cf. Proposition 1.9). Furthermore, the complex $\mathbb{R} h_{*} \mathcal{E}^{\bullet}$ is perfect over $W$. Restricting to $W$ our morphism of distinguished triangles, and recalling that cones of the morphisms

$$
\left.\mathcal{F}_{U,(1)}^{\bullet} \longrightarrow \mathcal{K}_{(1)}^{\bullet}\right|_{U} \quad \text { and }\left.\quad \mathcal{F}_{V,(1)}^{\bullet} \longrightarrow \mathcal{K}_{(1)}^{\bullet}\right|_{V}
$$

are coherent complexes over $U$ and $V$, one easily concludes that a cone of the morphism $\mathcal{F}^{\bullet} \rightarrow \mathcal{L}^{\bullet}$ is a coherent complex over $W$.

Denote this cone temporarily by $\mathcal{K}_{(2)}^{\bullet}$. Clearly, in order to show that the original complex $\mathcal{K}^{\bullet}$ is a direct summand of an object from $D^{\mathrm{b}}(Z \text {-qcoh })_{\mathrm{fl}-\mathrm{c}}$ in $\mathrm{D}^{\mathrm{b}}(Z$-qcoh $)$ (which is our goal), it suffices to check that the complex $\mathcal{K}_{(2)}^{\bullet}$ is as well. It also follows from the constructions that the restrictions of the complex $\mathcal{K}_{(2)}^{\bullet}$ to $U$ and $V$ belong to $\mathrm{D}^{\mathrm{b}}(U-\mathrm{qcoh})_{\mathrm{fl}-\mathrm{c}}$ and $\mathrm{D}^{\mathrm{b}}\left(V\right.$-qcoh $\left._{\mathrm{fl}-\mathrm{c}}\right)$, respectively. Dropping the lower index and redenoting $\mathcal{K}_{(2)}^{\bullet}$ simply by $\mathcal{K}^{\bullet}$, we are coming back to the situation in the beginning of the proof with the new knowledge that $\mathcal{K}^{\bullet}$ may be assumed to be a coherent complex over $W$. 
The next segment of our proof is based on the localization theory for coderived categories of quasicoherent sheaves on Noetherian schemes (similar to the ThomasonTrobaugh-Neeman theorem for the conventional derived categories, the difference being that arbitrary bounded complexes of coherent sheaves play the role of perfect complexes). What we need is a particular case of the theory developed in Section 1.10 (corresponding to the choice of the quasicoherent CDG-algebra $\mathcal{O}_{Z}$ over $Z$ ).

Specifically, it follows from Proposition 1.5(d) and Theorem 1.10 together with [Neeman 1996, Theorem 2.1(5)] that any morphism from an object of $D^{\mathrm{b}}(W$-coh) into a restriction to $W$ of an object $\mathcal{K}^{\bullet}$ from $\mathrm{D}^{\mathrm{b}}\left(Z\right.$-qcoh) (or even from $\mathrm{D}^{\mathrm{co}}(Z$-qcoh $\left.)\right)$ can be extended to a morphism to $\mathcal{K}^{\bullet}$ from an object of $\mathrm{D}^{\mathrm{b}}(Z$-coh). Applying this assertion to the identity morphism $\left.\left.\mathcal{K}^{\bullet}\right|_{W} \rightarrow \mathcal{K}^{\bullet}\right|_{W}$ in the above situation, we obtain a morphism $\mathcal{M}^{\bullet} \rightarrow \mathcal{K}^{\bullet}$ into $\mathcal{K}^{\bullet}$ from a coherent complex $\mathcal{M}^{\bullet}$ over $Z$ that is a quasiisomorphism over $W$. Passing to a cone of this morphism, we may assume $\mathcal{K} \bullet$ to be acyclic over $W$.

By Corollary 1.10, such a complex $\mathcal{K} \bullet$ is quasi-isomorphic to a (bounded) complex of quasicoherent sheaves on $Z$ whose terms are concentrated set-theoretically in the complement $Z \backslash W$. The latter is a disjoint union of two nonintersecting closed subsets in $Z$, namely, the complements $S=Z \backslash U$ and $T=Z \backslash V$. Now the complex $\mathcal{K}^{\bullet}$ decomposes into a direct sum of two complexes with set-theoretic supports inside $S$ and $T$, respectively.

One can consider the two direct summands separately. We have to show that any bounded complex of quasicoherent sheaves $\mathcal{K}^{\bullet}$ on $Z$, which is supported set-theoretically in $T$ and whose restriction to $U$ belongs to $\mathrm{D}^{\mathrm{b}}(U \text {-qcoh })_{\mathrm{fl}-\mathrm{c}}$, itself belongs to $\mathrm{D}^{\mathrm{b}}(Z \text {-qcoh })_{\mathrm{fl}-\mathrm{c}}$. Arguing as in the beginning of this proof, we have an object $\mathcal{G}^{\bullet} \in \mathrm{D}^{\mathrm{b}}(U$-qcoh fl $)$ together with a morphism $\left.\mathcal{G}^{\bullet} \rightarrow \mathcal{K}^{\bullet}\right|_{U}$ whose cone belongs to $\mathrm{D}^{\mathrm{b}}(U$-coh $)$. The restriction $\left.\mathcal{G}^{\bullet}\right|_{W}$ then belongs to both $\mathrm{D}^{\mathrm{b}}\left(W-\right.$ qcoh $\left._{\mathrm{fl}}\right)$ and $\mathrm{D}^{\mathrm{b}}(W$-coh), and is, therefore, a perfect complex on $W$.

Again by the Thomason-Trobaugh theorem, the object $\left.{ }^{\prime} \mathcal{G}^{\bullet}\right|_{W}$ can be extended to a perfect complex $\mathcal{H}^{\bullet}$ on $V$. A cocone of the morphism

$$
\left.\mathbb{R} j_{*}{ }^{\prime} \mathcal{G}^{\bullet} \oplus \mathbb{R} k_{*} \mathcal{H}^{\bullet} \longrightarrow \mathbb{R} h_{*}{ }^{\prime} \mathcal{G}^{\bullet}\right|_{W}
$$

provides an object $\mathcal{F}^{\bullet} \in \mathrm{D}^{\mathrm{b}}\left(Z\right.$-qcoh fl $\left._{\mathrm{fl}}\right)$ isomorphic to ' $\mathcal{G}^{\bullet}$ over $U$ and to $\mathcal{H}^{\bullet}$ over $V$. Now the morphism $\left.{ }^{\prime} \mathcal{G}^{\bullet} \rightarrow^{\prime} \mathcal{K}^{\bullet}\right|_{U}$ over $U$ extends uniquely to a morphism $\mathcal{F}^{\bullet} \rightarrow^{\prime} \mathcal{K}^{\bullet}$ over $Z$ since the set-theoretic support of ' $\mathcal{K}^{\bullet}$ is contained in a closed subset lying inside $U$. A cone of the morphism $\mathcal{F}^{\bullet} \rightarrow^{\prime} \mathcal{K}^{\bullet}$ is a coherent complex on $Z$ since it is so in restrictions to $U$ and $V$. Thus, the proposition is proven.

Now let $X$ be a separated Noetherian scheme of finite Krull dimension with enough vector bundles, $\mathcal{L}$ be a line bundle on $X$, and $w \in \mathcal{L}(X)$ be a locally nonzero-dividing potential. Let $X_{0} \subset X$ be the zero locus of $w$. 
Corollary 3.2. Let $X=U \cap V$ be a covering by two open subschemes. Then any object of $\mathrm{D}^{\mathrm{co}}\left((X, \mathcal{L}, w)\right.$-qcoh $\left.\mathrm{fl}_{\mathrm{fl}}\right)$ whose restrictions to $U$ and $V$ belong to the full triangulated subcategories

$$
\begin{gathered}
\mathrm{D}^{\text {abs }}\left(\left(U,\left.\mathcal{L}\right|_{U},\left.w\right|_{U}\right)-\operatorname{coh}_{\text {If }}\right) \subset \mathrm{D}^{\mathrm{co}}\left(\left(U,\left.\mathcal{L}\right|_{U},\left.w\right|_{U}\right)-\mathrm{qcoh}_{\mathrm{fl}}\right), \\
\mathrm{D}^{\mathrm{abs}}\left(\left(V,\left.\mathcal{L}\right|_{V},\left.w\right|_{V}\right)-\operatorname{coh}_{\mathrm{If}}\right) \subset \mathrm{D}^{\mathrm{co}}\left(\left(V,\left.\mathcal{L}\right|_{V},\left.w\right|_{V}\right)-\mathrm{qcoh}_{\mathrm{fl}}\right),
\end{gathered}
$$

respectively, is a direct summand of an object from the full triangulated subcategory $\mathrm{D}^{\text {abs }}\left((X, \mathcal{L}, w)-\operatorname{coh}_{\text {If }}\right) \subset \mathrm{D}^{\mathrm{co}}\left((X, \mathcal{L}, w)-\mathrm{qcoh}_{\mathrm{fl}}\right)$.

Proof. By Proposition 2.8, the category $\mathrm{D}^{\mathrm{co}}\left((X, \mathcal{L}, w)-\mathrm{qcoh}_{\mathrm{fl}}\right)$ is a full triangulated subcategory of the triangulated category $\mathrm{D}_{\text {Sing }}^{\prime}\left(X_{0}\right)$. The (essential) intersection of the full subcategories $\mathrm{D}^{\mathrm{co}}\left((X, \mathcal{L}, w)\right.$-qcoh $\left.\mathrm{fl}_{\mathrm{fl}}\right)$ and $\mathrm{D}_{\text {Sing }}^{\mathrm{b}}\left(X_{0}\right)$ in $\mathrm{D}_{\text {Sing }}^{\prime}\left(X_{0}\right)$ is the triangulated category $\mathrm{D}^{\text {abs }}\left((X, \mathcal{L}, w)\right.$-coh lf $\left._{\mathrm{f}}\right)$.

Indeed, an object of $\mathcal{F} \in \mathrm{D}_{\text {Sing }}^{\mathrm{b}}\left(X_{0}\right)$ belongs to $\mathrm{D}^{\text {abs }}\left((X, \mathcal{L}, w)\right.$-coh $\left.{ }_{\text {If }}\right)$ if and only if the object $i_{\circ} \mathcal{F}$ vanishes in $\mathrm{D}_{\text {Sing }}^{\mathrm{b}}(X)$ (Theorem 2.7); an object $\mathcal{F} \in \mathrm{D}_{\text {Sing }}^{\prime}\left(X_{0}\right)$ belongs to $\mathrm{D}^{\mathrm{CO}}\left((X, \mathcal{L}, w)\right.$-qcoh $\left.\mathrm{fl}_{\mathrm{fl}}\right)$ if and only if the object $i_{\circ} \mathcal{F}$ vanishes in $\mathrm{D}_{\text {Sing }}^{\prime}(X)$ (Proposition 2.8); and the functor $\mathrm{D}_{\text {Sing }}^{\mathrm{b}}(X) \rightarrow \mathrm{D}_{\text {Sing }}^{\prime}(X)$ is fully faithful.

Moreover, the (essential) intersection of $\mathrm{D}^{\mathrm{co}}\left((X, \mathcal{L}, w)\right.$-qcoh $\left.\mathrm{fl}_{\mathrm{fl}}\right)$ with the thick envelope of $\mathrm{D}_{\text {Sing }}^{\mathrm{b}}\left(X_{0}\right)$ in $\mathrm{D}_{\text {Sing }}^{\prime}\left(X_{0}\right)$ is the thick envelope of $\mathrm{D}^{\text {abs }}\left((X, \mathcal{L}, w)\right.$-coh $\left.{ }_{\text {If }}\right)$ in $\mathrm{D}_{\text {Sing }}^{\prime}\left(X_{0}\right)$. Indeed, let $\mathcal{M}$ be an object of the intersection; then $\mathcal{M} \oplus \mathcal{M}[1]$ belongs to both $\mathrm{D}^{\mathrm{co}}\left((X, \mathcal{L}, w)\right.$-qcoh $\left._{\mathrm{fl}}\right)$ and $\mathrm{D}_{\text {Sing }}^{\mathrm{b}}\left(X_{0}\right)$, hence also to $\mathrm{D}^{\text {abs }}\left((X, \mathcal{L}, w)\right.$-coh $\left.\mathrm{C}_{\mathrm{ff}}\right)$, and consequently $\mathcal{M}$ belongs to the thick envelope of $D^{\text {abs }}\left((X, \mathcal{L}, w)\right.$-coh $\left.{ }_{\text {lf }}\right)$.

Now let $\mathcal{K}$ be our object of $\mathrm{D}^{\mathrm{co}}\left((X, \mathcal{L}, w)\right.$-qcoh $\left.\mathrm{fl}_{\mathrm{fl}}\right)$; it can be also viewed as an object of $\mathrm{D}_{\text {Sing }}^{\prime}\left(X_{0}\right)$. If its restrictions to $U$ and $V$ belong to $\mathrm{D}^{\text {abs }}\left(\left(U,\left.\mathcal{L}\right|_{U},\left.w\right|_{U}\right)\right.$-coh $\left.{ }_{\text {If }}\right)$ and $\mathrm{D}^{\mathrm{abs}}\left(\left(V,\left.\mathcal{L}\right|_{V},\left.w\right|_{V}\right)\right.$-coh $\left.{ }_{\text {If }}\right)$, they also belong to $\mathrm{D}_{\text {Sing }}^{\mathrm{b}}\left(U_{0}\right) \subset \mathrm{D}_{\text {Sing }}^{\prime}\left(U_{0}\right)$ and $\mathrm{D}_{\text {Sing }}^{\mathrm{b}}\left(V_{0}\right) \subset \mathrm{D}_{\text {Sing }}^{\prime}\left(V_{0}\right)$ (where we set $U_{0}=U \cap X_{0}$ and $\left.V_{0}=V \cap X_{0}\right)$.

Applying Proposition 3.2, we can conclude that $\mathcal{K}$ belongs to the thick envelope of $\mathrm{D}_{\text {Sing }}^{\mathrm{b}}\left(X_{0}\right)$ in $\mathrm{D}_{\text {Sing }}^{\prime}\left(X_{0}\right)$. The assertion of Corollary 3.2 follows from the above.

Assume additionally that the scheme $X$ admits a dualizing complex $\mathcal{D}_{X}^{\bullet}$.

Theorem 3.2. Let $X=U \cap V$ be a covering by two open subschemes. Then any object of $\mathrm{D}^{\mathrm{abs}}((X, \mathcal{L}, w)$-coh) whose restrictions to $U$ and $V$ belong to the thick envelopes of the triangulated subcategories

$$
\begin{gathered}
\mathrm{D}^{\text {abs }}\left(\left(U,\left.\mathcal{L}\right|_{U},\left.w\right|_{U}\right)-\operatorname{coh}_{\text {If }}\right) \subset \mathrm{D}^{\text {abs }}\left(\left(U,\left.\mathcal{L}\right|_{U},\left.w\right|_{U}\right) \text {-coh }\right), \\
\mathrm{D}^{\text {abs }}\left(\left(V,\left.\mathcal{L}\right|_{V},\left.w\right|_{V}\right)-\operatorname{coh}_{\text {If }}\right) \subset \mathrm{D}^{\text {abs }}\left(\left(V, \mathcal{L}_{V},\left.w\right|_{V}\right) \text {-coh }\right)
\end{gathered}
$$

itself belongs to the thick envelope of the triangulated subcategory

$$
\mathrm{D}^{\text {abs }}\left((X, \mathcal{L}, w) \text {-coh }{ }_{\text {If }}\right) \subset \mathrm{D}^{\text {abs }}((X, \mathcal{L}, w) \text {-coh }) .
$$

Proof. The argument is based on the Serre-Grothendieck duality theory for matrix factorizations as developed in Section 2.5, which allows us to reduce the question to 
the result of Corollary 3.2. Specifically, let $\mathcal{M}$ be our coherent matrix factorization over $X$. Replacing, if necessary, $\mathcal{M}$ with $\mathcal{M} \oplus \mathcal{M}[1]$, we may assume the restrictions of $\mathcal{M}$ to $U$ and $V$ to be isomorphic to locally free matrix factorizations of finite rank.

Let us apply the construction of functor

$$
\Omega: \mathrm{D}^{\mathrm{abs}}((X, \mathcal{L}, w)-\mathrm{coh})^{\mathrm{op}} \longrightarrow \mathrm{D}^{\mathrm{co}}\left((X, \mathcal{L},-w)-\mathrm{qcoh}_{\mathrm{fl}}\right)
$$

from Section 2.5 to the matrix factorization $\mathcal{M}$. That is, we pick a left resolution of $\mathcal{M}$ by locally free matrix factorizations of finite rank, dualize by applying $\operatorname{Hom}_{X \text {-qc }}\left(-, \mathcal{O}_{X}\right)$, and totalize using infinite direct sums. By Corollary 2.5 , the functor $\Omega$ is fully faithful; it also identifies $\mathrm{D}^{\mathrm{abs}}\left((X, \mathcal{L}, w) \text {-coh }{ }_{\mathrm{lf}}\right)^{\mathrm{op}}$ with $\mathrm{D}^{\text {abs }}\left((X, \mathcal{L},-w)\right.$-coh $\left.{ }_{\mathrm{lf}}\right)$. Hence it suffices to check that the matrix factorization $\Omega(\mathcal{M})$ belongs to the thick envelope of $\mathrm{D}^{\text {abs }}\left((X, \mathcal{L},-w)-\operatorname{coh}_{\mathrm{lf}}\right)$ in $\mathrm{D}^{\mathrm{co}}\left((X, \mathcal{L},-w)\right.$-qcoh $\left.{ }_{\text {If }}\right)$. But we know as much from Corollary 3.2.

3.3. Nonlocalization of local freeness. The lack of a workable notion of the conventional derived category (as opposed to the coderived category) for quasicoherent matrix factorizations stands in the way of a direct extension of the ThomasonTrobaugh-Neeman localization theorem for perfect complexes [Thomason and Trobaugh 1990; Neeman 1992; 1996] to locally free matrix factorizations of finite rank. We have seen in Section 1.10 how the localization theory can be developed for coherent matrix factorizations. In this section we demonstrate a counterexample showing that the localization theory, in its conventional form, actually does not hold for locally free matrix factorizations.

In other words, the restriction

$$
\mathrm{D}^{\mathrm{abs}}\left((X, \mathcal{L}, w)-\operatorname{coh}_{\text {If }}\right) \longrightarrow \mathrm{D}^{\mathrm{abs}}\left(\left(U,\left.\mathcal{L}\right|_{U},\left.w\right|_{U}\right)-\operatorname{coh}_{\mathrm{If}}\right)
$$

for an open subscheme $U \subset X$ is not always a Verdier quotient functor, even up to the direct summands. Moreover, the triangulated category $\mathrm{D}^{\mathrm{abs}}\left((X, \mathcal{L}, w)\right.$-coh $\left.\mathrm{co}_{\mathrm{ff}}\right)$ may fail to be generated by a single object, unlike in the case of the categories of perfect complexes on quasicompact quasiseparated schemes.

All the potentials in our example will be simply regular functions, i.e., sections of the trivial line bundle $\mathcal{O}_{X}$ or $\mathcal{O}_{U}$, etc.; so we drop the line bundle $\mathcal{L}$ from our notation in the rest of the section and write simply $\mathrm{D}^{\mathrm{abs}}\left((X, w)-\right.$ coh $\left._{\mathrm{If}}\right)$ or $\mathrm{D}^{\mathrm{abs}}((X, w)$-coh), etc. For simplicity, we will work over the basic field of complex numbers $\mathbb{C}$.

Consider the 3-dimensional affine quadratic cone

$$
X=\{x y=z w\} \subset \mathbb{A}^{4}=\operatorname{Spec} \mathbb{C}[x, y, z, w] .
$$

Further, let us take the open subset

$$
U=\{z \neq 0\} \subset X .
$$


Clearly, we have an isomorphism of pairs (algebraic variety, regular function on it)

$$
(U, w) \stackrel{\sim}{\longrightarrow}\left(\mathbb{A}_{t_{1}, t_{2}}^{2} \times \mathbb{G}_{m}, t_{1} t_{2}\right), \quad(x, y, z, w) \longmapsto\left(\left(x, \frac{y}{z}\right), z\right),
$$

where we denote $\mathbb{A}_{t_{1}, t_{2}}^{2}=\operatorname{Spec} \mathbb{C}\left[t_{1}, t_{2}\right]$ and, as usual, $\mathbb{G}_{m}=\mathbb{A}^{1} \backslash\{0\}$.

Lemma 3.3. (a) We have a natural equivalence of triangulated categories

$$
\mathrm{D}^{\mathrm{abs}}((U, w)-\mathrm{coh}) \simeq \mathrm{D}^{\mathrm{abs}}\left(\left(\mathbb{G}_{m}, 0\right)-\mathrm{coh}\right) .
$$

(b) The restriction functor

$$
\mathrm{D}^{\mathrm{abs}}((X, w)-\mathrm{coh}) \longrightarrow \mathrm{D}^{\mathrm{abs}}((U, w)-\mathrm{coh})
$$

is an equivalence.

Here the category of matrix factorizations of the zero potential $\mathrm{D}^{\mathrm{abs}}((Y, 0)$-coh) is, of course, simply the derived category of 2-periodic complexes of coherent sheaves on a smooth variety $Y$.

Proof. Part (a): By (1), we have the equivalence

$$
\mathrm{D}^{\mathrm{abs}}((U, w)-\mathrm{coh}) \simeq \mathrm{D}^{\mathrm{abs}}\left(\left(\mathbb{A}_{t_{1}, t_{2}}^{2} \times \mathbb{G}_{m}, t_{1} t_{2}\right)-\mathrm{coh}\right) .
$$

By Knörrer periodicity (cf. [Orlov 2006, Theorem 3.1]), we have the equivalence

$$
\mathrm{D}^{\mathrm{abs}}\left(\left(\mathbb{A}_{t_{1}, t_{2}}^{2} \times \mathbb{G}_{m}, t_{1} t_{2}\right)-\mathrm{coh}\right) \simeq \mathrm{D}^{\mathrm{abs}}\left(\left(\mathbb{G}_{m}, 0\right)-\mathrm{coh}\right) .
$$

Part (b): Let us put $D=X \backslash U$. By Theorem 1.10(b) (see also Section 3.1), we have the short exact sequence of triangulated categories

$$
0 \longrightarrow \mathrm{D}_{D}^{\mathrm{abs}}((X, w) \text {-coh }) \longrightarrow \mathrm{D}^{\mathrm{abs}}((X, w) \text {-coh }) \longrightarrow \mathrm{D}^{\mathrm{abs}}((U, w) \text {-coh }) \longrightarrow 0
$$

Thus, we need to show that the category $\mathrm{D}_{D}^{\text {abs }}((X, w))$-coh) is zero. It suffices to check that the category $\mathrm{D}^{\text {abs }}((D, w)$-coh) is zero.

Let us put $S=\{x y=0\} \subset \mathbb{A}^{2}$. Then we have the isomorphism

$$
(D, w) \stackrel{\sim}{\longrightarrow}\left(S \times \mathbb{A}_{t}^{1}, t\right), \quad(x, y, 0, w) \longmapsto((x, y), w) .
$$

Since $\mathrm{D}^{\mathrm{abs}}\left(\left(\mathbb{A}_{t}^{1}, t\right)\right.$-coh $)=0$, it follows that

$$
\mathrm{D}^{\mathrm{abs}}((D, w)-\mathrm{coh}) \simeq \mathrm{D}^{\mathrm{abs}}\left(\left(S \times \mathbb{A}_{t}^{1}, t\right)-\mathrm{coh}\right)=0 .
$$

Since $U$ is smooth, we have the equivalence

$$
\mathrm{D}^{\mathrm{abs}}\left((U, w)-\text { coh }_{\mathrm{If}}\right) \simeq \mathrm{D}^{\mathrm{abs}}((U, w)-\mathrm{coh}) .
$$

Now we turn to the category $\mathrm{D}^{\mathrm{abs}}\left((X, w)\right.$-coh $\left.{ }_{\mathrm{If}}\right)$. As usual, we put

$$
X_{0}=\{w=0\} \subset X .
$$


According to Theorem 2.7, the triangulated category $\mathrm{D}^{\text {abs }}\left((X, w)\right.$-coh $\left.\mathrm{If}_{\mathrm{f}}\right)$ is equivalent to the kernel of the direct image functor $i_{\circ}: \mathrm{D}_{\text {Sing }}^{\mathrm{b}}\left(X_{0}\right) \rightarrow \mathrm{D}_{\text {Sing }}^{\mathrm{b}}(X)$ acting between the triangulated categories of singularities of the schemes $X_{0}$ and $X$. This can be rephrased by saying that $\mathrm{D}^{\text {abs }}\left((X, w)\right.$-coh $\left.{ }_{\mathrm{If}}\right)$ is equivalent to the quotient category of the category of bounded complexes of coherent sheaves on $X_{0}$ whose direct images are perfect complexes on $X$ by the category of perfect complexes on $X_{0}$. Denoting the triangulated category of coherent complexes on $X_{0}$ whose direct images are perfect on $X$ by $\operatorname{Perf}\left(X_{0}, X\right) \subset \mathrm{D}^{\mathrm{b}}\left(X_{0}\right.$-coh), we have the equivalence of triangulated categories

$$
\mathrm{D}^{\mathrm{abs}}\left((X, w)-\mathrm{coh}_{\mathrm{lf}}\right) \simeq \operatorname{Perf}\left(X_{0}, X\right) / \operatorname{Perf}\left(X_{0}\right) .
$$

Note that we have the natural isomorphism

$$
X_{0} \simeq S \times \mathbb{A}^{1}, \quad(x, y, z, 0) \longmapsto((x, y), z) .
$$

It follows immediately that

$$
\mathrm{D}_{\text {Sing }}^{\mathrm{b}}\left(X_{0}\right) \simeq \mathrm{D}^{\mathrm{abs}}\left(\left(\mathbb{A}^{1}, 0\right) \text {-coh }\right) .
$$

Proposition 3.3. (a) We have the natural equivalence of triangulated categories

$$
\operatorname{Perf}\left(X_{0}, X\right) / \operatorname{Perf}\left(X_{0}\right) \simeq \mathrm{D}^{\mathrm{abs}}\left(\left(\mathbb{G}_{m}, 0\right) \text {-coh }\right)_{0 \text {-dim }},
$$

where $\mathrm{D}^{\mathrm{abs}}\left(\left(\mathbb{G}_{m}, 0\right) \text {-coh }\right)_{0 \text {-dim }} \subset \mathrm{D}^{\mathrm{abs}}\left(\left(\mathbb{G}_{m}, 0\right)\right.$-coh) is the subcategory of complexes with zero-dimensional support.

Moreover, we have a commutative diagram of fully faithful triangulated functors:

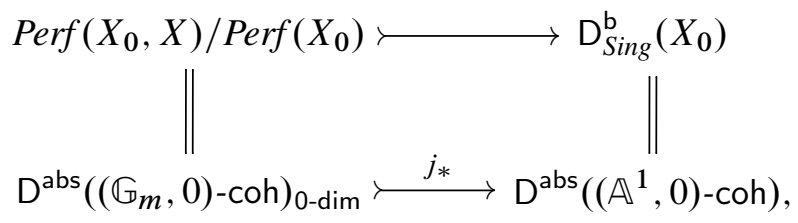

where $j: \mathbb{G}_{m} \rightarrow \mathbb{A}^{1}$ is the open embedding.

(b) We have a commutative diagram of fully faithful triangulated functors and equivalences:

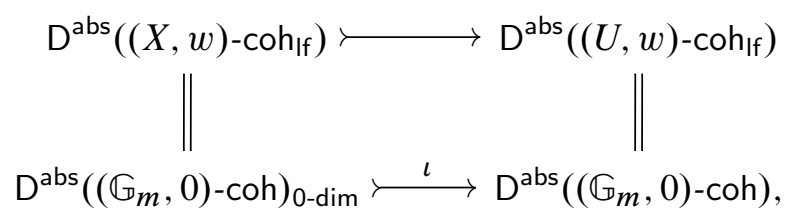

where $\mathrm{c}$ is the tautological embedding. 
Proof. Part (a): Indeed, from the equivalence (3) we have the natural fully faithful triangulated functor

$$
\operatorname{Perf}\left(X_{0}, X\right) / \operatorname{Perf}\left(X_{0}\right) \longrightarrow \mathrm{D}^{\mathrm{abs}}\left(\left(\mathbb{A}^{1}, 0\right)-\mathrm{coh}\right) .
$$

Let us denote by $T \subset D^{\text {abs }}\left(\left(\mathbb{A}^{1}, 0\right)\right.$-coh) the essential image of this functor. For each $z_{0} \in \mathbb{C} \backslash\{0\}$ we have a line $l_{z_{0}}:=\left\{y=0, z=z_{0}\right\} \subset X_{0}$. Since $l_{z_{0}} \subset U$ and $U$ is smooth, the coherent sheaf $\mathcal{O}_{l_{z_{0}}}$ is contained in $\operatorname{Perf}\left(X_{0}, X\right)$. Further, its image in $D_{\text {Sing }}^{\mathrm{b}}\left(X_{0}\right)$ corresponds to the skyscraper $\mathcal{O}_{z_{0}} \in \mathrm{D}^{\mathrm{abs}}\left(\left(\mathbb{A}^{1}, 0\right)\right.$-coh $)$ under the equivalence (3). It follows that the triangulated category $T$ contains $j_{*}\left(D^{\text {abs }}\left(\left(\mathbb{G}_{m}, 0\right) \text {-coh }\right)_{0-\text { dim }}\right)$ as a full subcategory.

Suppose that $\mathrm{T}$ is strictly bigger than $j_{*}\left(\mathrm{D}^{\mathrm{abs}}\left(\left(\mathbb{G}_{m}, 0\right) \text {-coh }\right)_{0 \text {-dim }}\right)$. Then it contains an object $\mathcal{F}_{0}=\mathcal{O}_{0} \oplus \mathcal{O}_{0}[1] \in \mathrm{D}^{\text {abs }}\left(\left(\mathbb{A}^{1}, 0\right)\right.$-coh), where $\mathcal{O}_{0}$ is the structure sheaf of the origin. Denote by $O \in X_{0}$ the origin $(0,0,0,0)$. Then the image of the coherent sheaf $\mathcal{O}_{O} \in X_{0}$-coh in $\mathrm{D}_{\text {Sing }}^{\mathrm{b}}\left(X_{0}\right)$ corresponds to $\mathcal{F}_{0}$ under the equivalence (3). But the object $\mathcal{O}_{O} \in \mathrm{D}^{\mathrm{b}}\left(X_{0}\right.$-coh) is not relatively perfect under the inclusion $X_{0} \rightarrow X$ (i.e., it does not belong to $\left.\operatorname{Perf}\left(X_{0}, X\right)\right)$ since $O$ is the singular point of $X$. We get a contradiction.

Thus, we have an equivalence $T \simeq j_{*}\left(\mathrm{D}^{\text {abs }}\left(\left(\mathbb{G}_{m}, 0\right) \text {-coh }\right)_{0 \text {-dim }}\right)$. This proves (a).

Part (b) follows immediately from part (a) and the equivalence (2).

In particular, we see that the functor $\mathrm{D}^{\text {abs }}\left((X, w)-\operatorname{coh}_{\mathrm{If}}\right) \rightarrow \mathrm{D}^{\mathrm{abs}}\left((U, w)-\operatorname{coh}_{\mathrm{If}}\right)$ is not essentially surjective, even up to the direct summands. Moreover, the triangulated category $\mathrm{D}^{\mathrm{abs}}\left((X, w)\right.$-coh $\left.{ }_{\mathrm{lf}}\right)$ does not even have a countable set of generators.

3.4. Pull-backs and push-forwards in singularity categories. Let $f: Y \rightarrow X$ be a morphism of separated Noetherian schemes with enough vector bundles. The morphism $f$ is said to have finite flat dimension if the derived inverse image functor $\mathbb{L} f^{*}: \mathrm{D}^{-}(X$-qcoh $) \rightarrow \mathrm{D}^{-}(Y$-qcoh $)$ takes $\mathrm{D}^{\mathrm{b}}(X$-qcoh $)$ to $\mathrm{D}^{\mathrm{b}}(Y$-qcoh $)$.

In this case, the functor $\mathbb{L} f^{*}$ induces the inverse image functors on the triangulated categories of singularities

$$
\begin{aligned}
& f^{\circ}: \mathrm{D}_{\text {Sing }}^{\prime}(X) \longrightarrow \mathrm{D}_{\text {Sing }}^{\prime}(Y) \\
& f^{\circ}: \mathrm{D}_{\text {Sing }}^{\mathrm{b}}(X) \longrightarrow \mathrm{D}_{\text {Sing }}^{\mathrm{b}}(Y) .
\end{aligned}
$$

Under the same assumption of finite flat dimension, the derived direct image functor $\mathbb{R} f_{*}: \mathrm{D}^{\mathrm{b}}(Y$-qcoh $) \rightarrow \mathrm{D}^{\mathrm{b}}(X$-qcoh $)$ takes $\mathrm{D}^{\mathrm{b}}\left(Y-\mathrm{q} c o h_{\mathrm{fl}}\right)$ to $\mathrm{D}^{\mathrm{b}}\left(X\right.$-qcoh $\left._{\mathrm{fl}}\right)$, as one can see by computing $\mathbb{R} f_{*}$ in terms of an affine covering of $Y$ in the spirit of the proof of Proposition 1.9. When the scheme $X$ has finite Krull dimension, one has $\mathrm{D}^{\mathrm{b}}\left(X\right.$-qcoh $\left._{\mathrm{fl}}\right)=\mathrm{D}^{\mathrm{b}}(X$-qcoh $\mathrm{If})$, so the functor $\mathbb{R} f_{*}$ induces the direct image functor

$$
f_{\circ}: \mathrm{D}_{\text {Sing }}^{\prime}(Y) \longrightarrow \mathrm{D}_{\text {Sing }}^{\prime}(X),
$$

which is right adjoint to $f^{\circ}$. 
Whenever the morphism $f$ is proper of finite type and has finite flat dimension, the functor $\mathbb{R} f_{*}$ takes $\mathrm{D}^{\mathrm{b}}\left(Y\right.$-coh) to $\mathrm{D}^{\mathrm{b}}(X$-coh) [Grothendieck 1961, Théorème 3.2.1] and induces the direct image functor

$$
f_{\circ}: \mathrm{D}_{\text {Sing }}^{\mathrm{b}}(Y) \longrightarrow \mathrm{D}_{\text {Sing }}^{\mathrm{b}}(X),
$$

which is right adjoint to $f^{\circ}$ [Orlov 2004, paragraphs before Proposition 1.14]. More generally, for a morphism $f$ of finite flat dimension and any closed subset $T \subset Y$ such that (a closed subscheme structure on) $T$ is proper of finite type over $X$, the functor $\mathbb{R} f_{*}$ takes $\mathrm{D}^{\mathrm{b}}(Y$-coh $T)$ to $\mathrm{D}^{\mathrm{b}}(X$-coh $)$ and induces the direct image functor

$$
f_{\circ}: \mathrm{D}_{\text {Sing }}^{\mathrm{b}}(Y, T) \longrightarrow \mathrm{D}_{\text {Sing }}^{\mathrm{b}}(X) .
$$

Indeed, the intersection of $\mathrm{D}^{\mathrm{b}}\left(X-\mathrm{qcoh}{ }_{\mathrm{fl}}\right)$ and $\mathrm{D}^{\mathrm{b}}(X$-coh $)$ in $\mathrm{D}^{\mathrm{b}}(X$-qcoh $)$ is equal to $\mathrm{D}^{\mathrm{b}}(X$-coh $\mathrm{lf})$, as any complex of finite flat dimension with bounded coherent cohomology is easily seen to be perfect.

Let $Z \subset X$ and $W \subset Y$ be closed subschemes such that $\mathcal{O}_{Z}$ is a perfect $\mathcal{O}_{X}$-module, $\mathcal{O}_{W}$ is a perfect $\mathcal{O}_{Y}$-module, and $f(W) \subset Z$. Assume that both morphisms $f: Y \rightarrow X$ and $\left.f\right|_{W}: W \rightarrow Z$ have finite flat dimensions. Then the derived inverse image functor $\left.\mathbb{L} f\right|_{W} ^{*}: \mathrm{D}^{\mathrm{b}}(Z$-qcoh $) \rightarrow \mathrm{D}^{\mathrm{b}}(W$-qcoh $)$ induces the inverse image functors on the triangulated categories of relative singularities

$$
\begin{aligned}
& f^{\circ}: \mathrm{D}_{\text {Sing }}^{\prime}(Z / X) \longrightarrow \mathrm{D}_{\text {Sing }}^{\prime}(W / Y) \\
& f^{\circ}: \mathrm{D}_{\text {Sing }}^{\mathrm{b}}(Z / X) \longrightarrow \mathrm{D}_{\text {Sing }}^{\mathrm{b}}(W / Y) .
\end{aligned}
$$

Now let $Z \subset X$ be a closed subscheme; set $W=Z \times_{X} Y$. Denote the closed embeddings $Z \rightarrow X$ and $W \rightarrow Y$ by $i$ and $i^{\prime}$, respectively; also let $f^{\prime}$ denote the morphism $\left.f\right|_{W}: W \rightarrow Z$. Assume that $W$ coincides with the derived product of $Z$ and $Y$ over $X$; i.e., $\mathbb{L} f^{*} i_{*} \mathcal{O}_{Z}=i_{*}^{\prime} \mathcal{O}_{W}$. Assume further that $i_{*} \mathcal{O}_{Z}$ is a perfect $\mathcal{O}_{X}$-module; then also $i_{*}^{\prime} \mathcal{O}_{W}$ is a perfect $\mathcal{O}_{Y}$-module.

For any $\mathcal{M} \in \mathrm{D}^{\mathrm{b}}(Y$-qcoh $)$, there is a natural morphism

$$
\phi_{\mathcal{M}}: \mathbb{L} i^{*} \mathbb{R} f_{*} \mathcal{M} \longrightarrow \mathbb{R} f_{*}^{\prime} \mathbb{L} i^{\prime *} \mathcal{M}
$$

in $\mathrm{D}^{\mathrm{b}}(Z$-qcoh). Using the projection formula for tensor products with perfect complexes, one easily checks that the morphism $i_{*} \phi_{\mathcal{M}}$ is an isomorphism. Hence, so is the morphism $\phi_{\mathcal{M}}$ since the functor $i_{*}$ does not annihilate any objects of the derived category. Hence we obtain the induced functor of direct image

$$
f_{\circ}: \mathrm{D}_{\text {Sing }}^{\prime}(W / Y) \longrightarrow \mathrm{D}_{\text {Sing }}^{\prime}(Z / X) .
$$

When the morphism $f$ is proper of finite type, there is also the induced functor

$$
f_{\circ}: \mathrm{D}_{\text {Sing }}^{\mathrm{b}}(W / Y) \longrightarrow \mathrm{D}_{\text {Sing }}^{\mathrm{b}}(Z / X) .
$$


Assume additionally that the morphism $f$ has finite flat dimension; then so does the morphism $f^{\prime}$. In this case, the functor $f_{0}: \mathrm{D}_{\text {Sing }}^{\prime}(W / Y) \rightarrow \mathrm{D}_{\text {Sing }}^{\prime}(Z / X)$ is right adjoint to the functor $f^{\circ}: \mathrm{D}_{\text {Sing }}^{\prime}(Z / X) \rightarrow \mathrm{D}_{\text {Sing }}^{\prime}(W / Y)$. When the morphism $f$ is proper of finite type, the functor $f_{\circ}: \mathrm{D}_{\text {Sing }}^{\mathrm{b}}(W / Y) \rightarrow \mathrm{D}_{\text {Sing }}^{\mathrm{b}}(Z / X)$ is right adjoint to the functor $f^{\circ}: \mathrm{D}_{\text {Sing }}^{\mathrm{b}}(Z / X) \rightarrow \mathrm{D}_{\text {Sing }}^{\mathrm{b}}(W / Y)$.

Remark 3.4. In the case when $Z$ is a Cartier divisor in $X$, we will construct the functor $f_{\circ}: \mathrm{D}_{\text {Sing }}^{\mathrm{b}}(W / Y) \rightarrow \mathrm{D}_{\text {Sing }}^{\mathrm{b}}(Z / X)$ under somewhat weaker assumptions below in Section 3.5. Namely, it will suffice that the morphism $f^{\prime}: W \rightarrow Z$ be proper of finite type, while the morphism $f: Y \rightarrow Z$ need not be. A generalization to the case of proper support will also be obtained.

3.5. Push-forwards of matrix factorizations. Let $f: Y \rightarrow X$ be a morphism of separated Noetherian schemes with enough vector bundles, $\mathcal{L}$ be a line bundle on $X$, and $w \in \mathcal{L}(X)$ be a section.

Set $\mathcal{B}_{X}=(X, \mathcal{L}, w)$ and $\mathcal{B}_{Y}=\left(Y, f^{*} \mathcal{L}, f^{*} w\right)$; then there is a natural morphism of CDG-algebras $\mathcal{B}_{X} \rightarrow \mathcal{B}_{Y}$ compatible with the morphism of schemes $f: Y \rightarrow X$. Therefore, according to Section 1.8, there are the derived inverse image functors

$$
\begin{gathered}
\mathbb{L} f^{*}: \mathrm{D}^{\mathrm{co}}\left((X, \mathcal{L}, w)-\mathrm{qcoh}_{\mathrm{ffd}}\right) \longrightarrow \mathrm{D}^{\mathrm{co}}\left(\left(Y, f^{*} \mathcal{L}, f^{*} w\right)-\text { qcoh }_{\mathrm{ffd}}\right), \\
\mathbb{L} f^{*}: \mathrm{D}^{\mathrm{abs}}\left((X, \mathcal{L}, w)-\operatorname{coh}_{\mathrm{ffd}}\right) \longrightarrow \mathrm{D}^{\text {abs }}\left(\left(Y, f^{*} \mathcal{L}, f^{*} w\right)-\text { coh }_{\mathrm{ffd}}\right)
\end{gathered}
$$

and the derived direct image functor

$$
\mathbb{R} f_{*}: \mathrm{D}^{\mathrm{co}}\left(\left(Y, f^{*} \mathcal{L}, f^{*} w\right) \text {-qcoh }\right) \longrightarrow \mathrm{D}^{\mathrm{co}}((X, \mathcal{L}, w) \text {-qcoh }) .
$$

The latter two functors are "partially adjoint" to each other.

Given a triangulated category $\mathrm{D}$, we denote by $\overline{\mathrm{D}}$ its idempotent completion. By [Balmer and Schlichting 2001, Section 1], the category $\bar{D}$ has the natural structure of a triangulated category.

Lemma 3.5. For any closed subset $T \subset Y$ such that (for a closed subscheme structure on $T$ ) the morphism $\left.f\right|_{T}: T \rightarrow X$ is proper of finite type, the functor $\mathbb{R} f_{*}$ takes the full subcategory $\mathrm{D}^{\mathrm{abs}}\left(\left(Y, f^{*} \mathcal{L}, f^{*} w\right)\right.$-coh $\left.\cos _{T}\right) \subset \mathrm{D}^{\mathrm{co}}\left(\left(Y, f^{*} \mathcal{L}, f^{*} w\right)\right.$-qcoh $)$ into the full subcategory $\mathrm{D}^{\mathrm{abs}}((X, \mathcal{L}, w)$-coh $) \subset \mathrm{D}^{\mathrm{co}}((X, \mathcal{L}, w)$-qcoh $)$, thus defining a triangulated functor of direct image

$$
\mathbb{R} f_{*}: \mathrm{D}^{\mathrm{abs}}\left(\left(Y, f^{*} \mathcal{L}, f^{*} w\right)-\operatorname{coh}_{T}\right) \longrightarrow \mathrm{D}^{\text {abs }}((X, \mathcal{L}, w) \text {-coh }) .
$$

Consequently, there is the triangulated functor

$$
\overline{\mathbb{R} f_{*}}: \overline{\mathrm{D}_{T}^{\mathrm{abs}}\left(\left(Y, f^{*} \mathcal{L}, f^{*} w\right)-\mathrm{coh}\right)} \longrightarrow \overline{\mathrm{D}^{\mathrm{abs}}((X, \mathcal{L}, w)-\mathrm{coh})} .
$$

Proof. We will use the construction of the functor

$$
\mathbb{R} f_{*}: \mathrm{D}^{\mathrm{co}}\left(\left(Y, f^{*} \mathcal{L}, f^{*} w\right) \text {-qcoh }\right) \longrightarrow \mathrm{D}^{\mathrm{co}}((X, \mathcal{L}, w) \text {-qcoh })
$$


similar to the one in the proof of Proposition 1.9 (see Remark 1.9). According to this construction, given a matrix factorization $\mathcal{M} \in\left(Y, f^{*} \mathcal{L}, f^{*} w\right)$-qcoh, the object $\mathbb{R} f_{*} \mathcal{M} \in \mathrm{D}^{\mathrm{co}}((X, \mathcal{L}, w)$-qcoh $)$ is represented by the total matrix factorization $\mathbb{R}_{\left\{U_{\alpha}\right\}} f_{*} \mathcal{M}$ of the finite Čech complex $f_{*} C_{\left\{U_{\alpha}\right\}}^{\bullet} \mathcal{M}$ of matrix factorizations on $X$. The derived functor of direct image of complexes of quasicoherent sheaves $\mathbb{R} f_{*}: \mathrm{D}^{\mathrm{b}}(Y$-qcoh $) \rightarrow \mathrm{D}^{\mathrm{b}}(X$-qcoh $)$ can be constructed in the same way.

By [Grothendieck 1961, Théorème 3.2.1], the latter functor takes $\mathrm{D}^{\mathrm{b}}\left(Y-\operatorname{coh}_{T}\right)$ into $\mathrm{D}^{\mathrm{b}}(X$-coh). Hence the cohomology matrix factorizations of the finite complex of matrix factorizations $f_{*} C_{\left\{U_{\alpha}\right\}}^{\bullet} \mathcal{M}$ belong to $(X, \mathcal{L}, w)$-coh when the matrix fac-

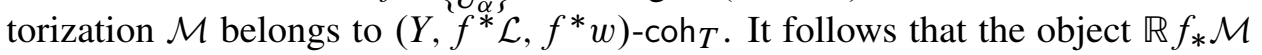
belongs to $\mathrm{D}^{\text {abs }}((X, \mathcal{L}, w)$-coh $) \subset \mathrm{D}^{\mathrm{co}}((X, \mathcal{L}, w)$-qcoh) in this case.

To prove the last assertion, it remains to apply Corollary 1.10(b).

Now assume that both morphisms of sheaves $w: \mathcal{O}_{X} \rightarrow \mathcal{L}$ and $f^{*} w: \mathcal{O}_{Y} \rightarrow f^{*} \mathcal{L}$ are injective. Let $X_{0} \subset X$ and $Y_{0} \subset Y$ denote the closed subschemes defined locally by the equations $w=0$ and $f^{*} w=0$, respectively. In this setting, we will compare the constructions of direct image functors for matrix factorizations and for the triangulated categories of relative singularities, and prove the assertions of Lemma 3.5 in a different way. Recall that in Section 3.4 we constructed the functor of direct image $f_{\circ}: \mathrm{D}_{\text {Sing }}^{\prime}\left(Y_{0} / Y\right) \rightarrow \mathrm{D}_{\text {Sing }}^{\prime}\left(X_{0} / X\right)$.

Proposition 3.5. (a) Whenever the morphism $f_{0}=\left.f\right|_{Y_{0}}: Y_{0} \rightarrow X_{0}$ is proper of finite type, the functor $\mathbb{R} f_{*}$ takes the full subcategory

$$
\mathrm{D}^{\text {abs }}\left(\left(Y, f^{*} \mathcal{L}, f^{*} w\right) \text {-coh }\right) \subset \mathrm{D}^{\mathrm{co}}\left(\left(Y, f^{*} \mathcal{L}, f^{*} w\right) \text {-qcoh }\right)
$$

into the full subcategory

$$
\mathrm{D}^{\text {abs }}((X, \mathcal{L}, w) \text {-coh }) \subset \mathrm{D}^{\mathrm{co}}((X, \mathcal{L}, w) \text {-qcoh }),
$$

thus defining a triangulated functor

$$
\mathbb{R} f_{*}: \mathrm{D}^{\text {abs }}\left(\left(Y, f^{*} \mathcal{L}, f^{*} w\right) \text {-coh }\right) \longrightarrow \mathrm{D}^{\text {abs }}((X, \mathcal{L}, w) \text {-coh }) .
$$

(b) For any closed subset $T \subset Y_{0}$ such that (for a closed subscheme structure on $T$ ) the morphism $\left.f_{0}\right|_{T}: T \rightarrow X_{0}$ is proper of finite type, the functor $f_{\circ}$ takes the full subcategory $\mathrm{D}_{\text {Sing }}^{\mathrm{b}}\left(Y_{0} / Y, T\right) \subset \mathrm{D}_{\text {Sing }}^{\prime}\left(Y_{0} / Y\right)$ into the full subcategory $\mathrm{D}_{\text {Sing }}^{\mathrm{b}}\left(X_{0} / X\right) \subset \mathrm{D}_{\text {Sing }}^{\prime}\left(X_{0} / X\right)$, thus defining a triangulated functor

$$
f_{\circ}: \mathrm{D}_{\text {Sing }}^{\mathrm{b}}\left(Y_{0} / Y, T\right) \longrightarrow \mathrm{D}_{\text {Sing }}^{\mathrm{b}}\left(X_{0} / X\right) \text {. }
$$

(c) The equivalences of categories $\mathrm{D}^{\mathrm{abs}}\left(\left(Y, f^{*} \mathcal{L}, f^{*} w\right)-\operatorname{coh}_{T}\right) \simeq \mathrm{D}_{\text {Sing }}^{\mathrm{b}}\left(X_{0} / X, T\right)$ from Proposition 3.1 (a) and $\mathrm{D}^{\mathrm{abs}}((X, \mathcal{L}, w)$-coh $) \simeq \mathrm{D}_{\text {Sing }}^{\mathrm{b}}\left(X_{0} / X\right)$ from Theorem 2.7 transform the direct image functor

$$
\mathbb{R} f_{*}: \mathrm{D}^{\mathrm{abs}}\left(\left(Y, f^{*} \mathcal{L}, f^{*} w\right)-\mathrm{coh}_{T}\right) \longrightarrow \mathrm{D}^{\mathrm{abs}}((X, \mathcal{L}, w)-\mathrm{coh})
$$

from Lemma 3.5 into the direct image functor $f_{\circ}$ from part $(\mathrm{b})$. 
Proof. Part (a) follows from Lemma 3.5 and Proposition 3.1(a), or alternatively, from part (b) and the proof of part (c) below. In part (b), the fact of key importance is that the functor $\mathrm{D}_{\text {Sing }}^{\mathrm{b}}\left(X_{0} / X\right) \rightarrow \mathrm{D}_{\text {Sing }}^{\prime}\left(X_{0} / X\right)$ is fully faithful (by Theorem 2.8). The functor $f_{\circ}$ takes $\mathrm{D}_{\text {Sing }}^{\mathrm{b}}\left(Y_{0} / Y, T\right)$ into $\mathrm{D}_{\text {Sing }}^{\mathrm{b}}\left(X_{0} / X\right)$ because the functor $\mathbb{R} f_{0 *}: \mathrm{D}^{\mathrm{b}}\left(Y_{0}\right.$-qcoh $) \rightarrow \mathrm{D}^{\mathrm{b}}\left(X_{0}\right.$-qcoh $)$ takes $\mathrm{D}^{\mathrm{b}}\left(Y_{0}\right.$-coh $\left.T\right)$ into $\mathrm{D}^{\mathrm{b}}\left(X_{0}\right.$-coh $)$ [Grothendieck 1961]. To prove part (c), we will check that the equivalences of categories from Theorem 2.8 transform the functor $\mathbb{R} f_{*}: \mathrm{D}^{\mathrm{co}}\left(\left(Y, f^{*} \mathcal{L}, f^{*} w\right)\right.$-qcoh) $\rightarrow$ $\mathrm{D}^{\mathrm{co}}\left((X, \mathcal{L}, w)\right.$-qcoh) into the functor $f_{\circ}: \mathrm{D}_{\text {Sing }}^{\prime}\left(Y_{0} / Y\right) \rightarrow \mathrm{D}_{\text {Sing }}^{\prime}\left(X_{0} / X\right)$. (Together with part (b) and Proposition 3.1(a), this will also provide another proof of Lemma 3.5.)

For this purpose, extend the functor

$$
\Upsilon_{Y}: \mathrm{D}^{\mathrm{b}}\left(Y_{0} \text {-qcoh }\right) \longrightarrow \mathrm{D}^{\mathrm{co}}\left(\left(Y, f^{*} \mathcal{L}, f^{*} w\right) \text {-qcoh }\right)
$$

to the functor $\widetilde{\Upsilon}_{Y}: \mathrm{D}^{+}\left(Y_{0}\right.$-qcoh $) \rightarrow \mathrm{D}^{\mathrm{co}}\left(\left(Y, f^{*} \mathcal{L}, f^{*} w\right)\right.$-qcoh $)$ in the obvious way (taking infinite direct sums of quasicoherent sheaves in the construction of the matrix factorization $\widetilde{\Upsilon}_{Y}\left(\mathcal{F}^{\bullet}\right)$ ). The functor $\widetilde{\Upsilon}_{Y}$ is well-defined since any boundedbelow acyclic complex of quasicoherent sheaves is coacyclic [Positselski 2010, Lemma 2.1]. Furthermore, the functor $\widetilde{\Upsilon}_{Y}$ can be presented as the composition of the "periodicity summation" functor $\mathrm{D}^{+}\left(Y_{0}\right.$-qcoh $) \rightarrow \mathrm{D}^{\mathrm{co}}\left(\left(Y_{0}, i^{\prime *} f^{*} \mathcal{L}, 0\right)\right.$-qcoh) taking values in the coderived category of quasicoherent matrix factorizations of the zero potential on $Y_{0}$, and the functor of direct image $i_{*}^{\prime}: \mathrm{D}^{\mathrm{co}}\left(\left(Y_{0}, i^{\prime *} f^{*} \mathcal{L}, 0\right)\right.$-qcoh $) \rightarrow$ $\mathrm{D}^{\mathrm{co}}\left(\left(Y, f^{*} \mathcal{L}, f^{*} w\right)\right.$-qcoh) with respect to the closed embedding $i^{\prime}$.

The functors

$$
\begin{gathered}
\mathbb{R} f_{0 *}: \mathrm{D}^{+}\left(Y_{0} \text {-qcoh }\right) \longrightarrow \mathrm{D}^{+}\left(X_{0} \text {-qcoh }\right), \\
\mathbb{R} f_{*}: \mathrm{D}^{\mathrm{co}}\left(\left(Y, f^{*} \mathcal{L}, f^{*} w\right)-\mathrm{qcoh}\right) \longrightarrow \mathrm{D}^{\mathrm{co}}((X, \mathcal{L}, w) \text {-qcoh })
\end{gathered}
$$

form a commutative diagram with the functors $\widetilde{\Upsilon}_{X}$ and $\widetilde{\Upsilon}_{Y}$. Indeed, the "periodicity summations" of bounded-below complexes of quasicoherent sheaves on $Y_{0}$ and $X_{0}$, taking injective resolutions to injective resolutions, obviously commute with the derived direct images with respect to $f^{\prime}$, as the direct image preserves infinite direct sums. Furthermore, the derived direct images of quasicoherent matrix factorizations are compatible with the compositions of morphisms of schemes (see Remark 1.8), and hence also commute with each other. It follows that the functors $\mathbb{R} f_{*}$ and $f_{\circ}$ agree as they should. (Alternatively, one can prove this in the way similar to the proof of Proposition 3.6 below.)

3.6. Push-forwards for morphisms of finite flat dimension. Let $f: Y \rightarrow X$ be a morphism of finite flat dimension between separated Noetherian schemes with enough vector bundles, $\mathcal{L}$ be a line bundle on $X$, and $w \in \mathcal{L}(X)$ be a section. As 
in Section 3.5, we have a natural morphism of CDG-algebras $\mathcal{B}_{X}=(X, \mathcal{L}, w) \rightarrow$ $\mathcal{B}_{Y}=\left(Y, f^{*} \mathcal{L}, f^{*} w\right)$ compatible with the morphism of schemes $Y \rightarrow X$.

The quasicoherent graded algebra $\mathcal{B}_{Y}$ has finite flat dimension over $\mathcal{B}_{X}$. Therefore, according to Section 1.9, there are derived inverse image functors

$$
\begin{aligned}
& \mathbb{L} f^{*}: \mathrm{D}^{\mathrm{co}}((X, \mathcal{L}, w) \text {-qcoh }) \longrightarrow \mathrm{D}^{\mathrm{co}}\left(\left(Y, f^{*} \mathcal{L}, f^{*} w\right) \text {-qcoh }\right) \\
& \mathbb{L} f^{*}: \mathrm{D}^{\mathrm{abs}}((X, \mathcal{L}, w) \text {-coh }) \longrightarrow \mathrm{D}^{\mathrm{abs}}\left(\left(Y, f^{*} \mathcal{L}, f^{*} w\right) \text {-coh }\right),
\end{aligned}
$$

the former of which is left adjoint to the functor

$$
\mathbb{R} f_{*}: \mathrm{D}^{\mathrm{co}}\left(\left(Y, f^{*} \mathcal{L}, f^{*} w\right) \text {-qcoh }\right) \longrightarrow \mathrm{D}^{\mathrm{co}}((X, \mathcal{L}, w) \text {-qcoh })
$$

from Section 3.5.

Furthermore, according to Proposition 1.9, there is a derived direct image functor $\mathbb{R} f_{*}: \mathrm{D}^{\mathrm{co}}\left(\left(Y, f^{*} \mathcal{L}, f^{*} w\right)-\right.$ qcoh $\left._{\mathrm{ffd}}\right) \simeq \mathrm{D}^{\mathrm{co}}\left(\left(Y, f^{*} \mathcal{L}, f^{*} w\right)-\mathrm{qcoh}_{\mathrm{fl}}\right)$

$$
\longrightarrow \mathrm{D}^{\mathrm{co}}((X, \mathcal{L}, w)-\mathrm{qcoh} \text { ffd }) \simeq \mathrm{D}^{\mathrm{co}}\left((X, \mathcal{L}, w)-\mathrm{qcoh}_{\mathrm{fl}}\right),
$$

which is right adjoint to the functor

$$
\mathbb{L} f^{*}: \mathrm{D}^{\mathrm{co}}\left((X, \mathcal{L}, w)-\text { qcoh }_{\mathrm{ffd}}\right) \longrightarrow \mathrm{D}^{\mathrm{co}}\left(\left(Y, f^{*} \mathcal{L}, f^{*} w\right)-\text { qcoh }_{\mathrm{ffd}}\right)
$$

from Section 3.5.

Now assume that $X$ and $Y$ have finite Krull dimensions. Recall that the natural triangulated functors $\mathrm{D}^{\mathrm{abs}}\left((X, \mathcal{L}, w)-\operatorname{coh}_{\mathrm{If}}\right) \rightarrow \mathrm{D}^{\mathrm{co}}\left((X, \mathcal{L}, w)\right.$-qcoh $\left.\mathrm{cos}_{\mathrm{fl}}\right)$ and $\mathrm{D}^{\text {abs }}\left(\left(Y, f^{*} \mathcal{L}, f^{*} w\right)\right.$-coh If $) \rightarrow \mathrm{D}^{\mathrm{co}}\left(\left(Y, f^{*} \mathcal{L}, f^{*} w\right)\right.$-qcoh fl $)$ are fully faithful by Corollary 2.3(e) and (j).

As in the second half of Section 3.5, assume that both morphisms of sheaves $w: \mathcal{O}_{X} \rightarrow \mathcal{L}$ and $f^{*} w: \mathcal{O}_{Y} \rightarrow f^{*} \mathcal{L}$ are injective, and denote by $f_{0}: Y_{0} \rightarrow X_{0}$ the induced morphism between the zero loci schemes of $f^{*} w$ and $w$. Since the morphism $f$ has finite flat dimension, so does the morphism $f_{0}$.

Proposition 3.6. (a) Whenever the morphism $f_{0}$ is proper of finite type, the functor

$$
\mathbb{R} f_{*}: \mathrm{D}^{\mathrm{co}}\left(\left(Y, f^{*} \mathcal{L}, f^{*} w\right)-\mathrm{qcoh} \mathrm{fl}_{\mathrm{fl}}\right) \longrightarrow \mathrm{D}^{\mathrm{co}}\left((X, \mathcal{L}, w)-\mathrm{qcoh}_{\mathrm{fl}}\right)
$$

takes the full subcategory $\mathrm{D}^{\mathrm{abs}}\left(\left(Y, f^{*} \mathcal{L}, f^{*} w\right)\right.$-coh $\left.\operatorname{cof}_{\mathrm{ff}}\right) \subset \mathrm{D}^{\mathrm{co}}\left(\left(Y, f^{*} \mathcal{L}, f^{*} w\right)\right.$-qcoh $\left.\cos _{\mathrm{fl}}\right)$ into the full subcategory $\mathrm{D}^{\mathrm{abs}}\left((X, \mathcal{L}, w)-\operatorname{coh}_{\mathrm{If}}\right) \subset \mathrm{D}^{\mathrm{co}}\left((X, \mathcal{L}, w)-\mathrm{qcoh}_{\mathrm{fl}}\right)$. Besides, the functor

$$
f_{0 \circ}: \mathrm{D}_{\text {Sing }}^{\mathrm{b}}\left(Y_{0}\right) \longrightarrow \mathrm{D}_{\text {Sing }}^{\mathrm{b}}\left(X_{0}\right)
$$

takes the full subcategory $\mathrm{D}^{\text {abs }}\left(\left(Y, f^{*} \mathcal{L}, f^{*} w\right)\right.$-coh $\left.\mathrm{If}_{\mathrm{f}}\right) \subset \mathrm{D}_{\text {Sing }}^{\mathrm{b}}\left(Y_{0}\right)$ into the full subcategory $\mathrm{D}^{\mathrm{abs}}\left((X, \mathcal{L}, w)-\mathrm{coh}_{\mathrm{If}}\right) \subset \mathrm{D}_{\text {Sing }}^{\mathrm{b}}\left(X_{0}\right)$. Both restrictions define the same triangulated functor

$$
\mathbb{R} f_{*}: \mathrm{D}^{\mathrm{abs}}\left(\left(Y, f^{*} \mathcal{L}, f^{*} w\right)-\mathrm{coh}_{\mathrm{lf}}\right) \longrightarrow \mathrm{D}^{\mathrm{abs}}\left((X, \mathcal{L}, w)-\mathrm{coh}_{\mathrm{ff}}\right) .
$$


(b) For any closed subset $T \subset Y_{0}$ such that (for a closed subscheme structure on $T$ ) the morphism $\left.f_{0}\right|_{T}: T \rightarrow X_{0}$ is proper of finite type, the functor

$$
\mathbb{R} f_{*}: \mathrm{D}^{\mathrm{co}}\left(\left(Y, f^{*} \mathcal{L}, f^{*} w\right)-\mathrm{qcoh} \mathrm{fl}_{\mathrm{fl}}\right) \longrightarrow \mathrm{D}^{\mathrm{co}}\left((X, \mathcal{L}, w)-\mathrm{qcoh}_{\mathrm{fl}}\right)
$$

takes the full subcategory $\mathrm{D}_{T}^{\text {abs }}\left(\left(Y, f^{*} \mathcal{L}, f^{*} w\right)\right.$-coh $\left.\operatorname{cof}_{\mathrm{ff}}\right) \subset \mathrm{D}^{\mathrm{co}}\left(\left(Y, f^{*} \mathcal{L}, f^{*} w\right)\right.$-qcoh $\left.\mathrm{ql}_{\mathrm{fl}}\right)$ into the thick envelope of the full subcategory

$$
\mathrm{D}^{\text {abs }}\left((X, \mathcal{L}, w)-\operatorname{coh}_{\mid \mathrm{f}}\right) \subset \mathrm{D}^{\mathrm{co}}\left((X, \mathcal{L}, w)-\mathrm{qcoh}_{\mathrm{fl}}\right) .
$$

Besides, the triangulated functor

$$
\overline{f_{0 \circ}}: \overline{\mathrm{D}_{\text {Sing }}^{\mathrm{b}}\left(Y_{0}, T\right)} \longrightarrow \overline{\mathrm{D}_{\text {Sing }}^{\mathrm{b}}\left(X_{0}\right)}
$$

takes the full subcategory $\mathrm{D}_{T}^{\mathrm{abs}}\left(\left(Y, f^{*} \mathcal{L}, f^{*} w\right)\right.$-coh $\left.\mathrm{cof}_{\mathrm{f}}\right) \subset \overline{\mathrm{D}_{\text {Sing }}^{\mathrm{b}}\left(Y_{0}, T\right)}$ into the thick envelope of the full subcategory

$$
\mathrm{D}^{\mathrm{abs}}\left((X, \mathcal{L}, w)-\operatorname{coh}_{\mathrm{If}}\right) \subset \overline{\mathrm{D}_{\text {Sing }}^{\mathrm{b}}\left(X_{0}\right)} .
$$

Both restrictions define the same triangulated functor

$$
\overline{\mathbb{R} f_{*}}: \overline{\mathrm{D}_{T}^{\text {abs }}\left(\left(Y, f^{*} \mathcal{L}, f^{*} w\right)-\mathrm{coh}_{\text {If }}\right)} \longrightarrow \overline{\mathrm{D}^{\text {abs }}\left((X, \mathcal{L}, w)-\text { coh }_{\text {If }}\right)} .
$$

Proof. Both categories $\mathrm{D}^{\mathrm{co}}\left((X, \mathcal{L}, w)\right.$-qcoh $\left.\mathrm{fl}_{\mathrm{fl}}\right)$ and $\mathrm{D}_{\text {Sing }}^{\mathrm{b}}\left(X_{0}\right)$ are full triangulated subcategories of the triangulated category $\mathrm{D}_{\text {Sing }}^{\prime}\left(X_{0}\right)$ (see Proposition 2.8 and [Orlov 2004, Proposition 1.13]). According to the proof of Corollary 3.2, the intersection of $\mathrm{D}^{\mathrm{co}}\left((X, \mathcal{L}, w)\right.$-qcoh $\left.{ }_{\mathrm{fl}}\right)$ with (the thick envelope of) $\mathrm{D}_{\text {Sing }}^{\mathrm{b}}\left(X_{0}\right)$ in $\mathrm{D}_{\text {Sing }}^{\prime}\left(X_{0}\right)$ (is the thick envelope of) the subcategory $\mathrm{D}^{\text {abs }}\left((X, \mathcal{L}, w)\right.$-coh If $\left.^{\prime}\right) \subset \mathrm{D}_{\text {Sing }}^{\prime}\left(X_{0}\right)$.

Thus it suffices to show that the direct image functor

$$
\mathbb{R} f_{*}: \mathrm{D}^{\mathrm{co}}((X, \mathcal{L}, w)-\mathrm{qcoh} f \mathrm{fl}) \longrightarrow \mathrm{D}^{\mathrm{co}}\left((X, \mathcal{L}, w)-\mathrm{qcoh}_{\mathrm{fl}}\right)
$$

agrees with the direct image functor $f_{0 \circ}: \mathrm{D}_{\text {Sing }}^{\prime}\left(Y_{0}\right) \rightarrow \mathrm{D}_{\text {Sing }}^{\prime}\left(X_{0}\right)$. The latter assertion does not depend on any properness assumptions.

Recall that the derived functor $\mathbb{R} f_{*}$ was constructed in the proof of Proposition 1.9 in terms of the Čech complex whose terms are direct sums of the CDG-modules $\left.\left.f\right|_{V * \mathcal{M}} \mathcal{M}\right|_{V}$, where $\mathcal{M} \in \mathrm{D}^{\mathrm{co}}\left(\left(Y, f^{*} \mathcal{L}, f^{*} w\right)\right.$-q $\left.\operatorname{coh}_{\text {ffd }}\right)$ and $V \subset Y$. The derived direct image $\mathbb{R} f_{0 *}: \mathrm{D}^{\mathrm{b}}\left(Y_{0}\right.$-qcoh $) \rightarrow \mathrm{D}^{\mathrm{b}}\left(X_{0}\right.$-qcoh $)$ can be constructed in the similar way; moreover, one can use for this purpose the restriction to $Y_{0}$ of an affine open covering $U_{\alpha}$ of the scheme $Y$.

We will make use of the flat dimension analogue of Corollary 2.6(d). Let $\widetilde{\Sigma}_{X}^{\prime}$ and $\tilde{\Sigma}_{Y}^{\prime}$ denote the obvious extensions of the functors $\Sigma^{\prime}$ from $(X, \mathcal{L}, w)$-qcoh ${ }_{\text {lf }}$ to the category of $w$-flat matrix factorizations of finite flat dimension $(X, \mathcal{L}, w)$-qcoh $w$-flnffd and from $\left(Y, f^{*} \mathcal{L}, f^{*} w\right)$-qcoh If $_{\text {If }}\left(Y, f^{*} \mathcal{L}, f^{*} w\right)$-qcoh $f^{*} w$-fl $\cap$ ffd (see the proofs of Proposition 2.8 and Theorem 2.7). Notice that the direct image functors $\left.f\right|_{V *}$ 
take $f^{*} w$-flat sheaves to $w$-flat sheaves and $\left(V,\left.f^{*} \mathcal{L}\right|_{V},\left.f^{*} w\right|_{V}\right)$-qcoh $f^{*} w$-fl $\cap$ ffd to $(X, \mathcal{L}, w)$-qcoh $w$-finffd .

Let $\mathcal{N}$ be a matrix factorization from $\left(Y, f^{*} \mathcal{L}, f^{*} w\right)$-qcoh $f^{*} w$-f। $\cap$ ffd. Since the open subschemes $V$ are presumed to be affine, there are natural isomorphisms

$$
\left.\left.\widetilde{\Sigma}_{X}^{\prime}\left(\left.\left.f\right|_{V * \mathcal{N}}\right|_{V}\right) \simeq f_{0}\right|_{V \cap Y_{0} *} \widetilde{\Sigma}_{Y}^{\prime}(\mathcal{N})\right|_{V \cap Y_{0}}
$$

of quasicoherent sheaves on $X_{0}$. Now it remains to use the next lemma.

Lemma 3.6. Let $\mathcal{M}^{-n} \rightarrow \cdots \rightarrow \mathcal{M}^{N}$ be a finite complex of matrix factorizations from $(X, \mathcal{L}, w)$-qcoh $w$-finffd and $\mathcal{M}$ be its totalization. Then the complex $\widetilde{\Sigma}^{\prime}\left(\mathcal{M}^{-n}\right) \rightarrow \cdots \rightarrow \widetilde{\Sigma}^{\prime}\left(\mathcal{M}^{N}\right)$ and the quasicoherent sheaf $\widetilde{\Sigma}^{\prime}(\mathcal{M})$ on $X_{0}$ represent naturally isomorphic objects in the triangulated category of singularities $\mathrm{D}_{\text {Sing }}^{\prime}\left(X_{0}\right)$. The same applies to a finite complex of matrix factorizations from $(X, \mathcal{L}, w)$-qcoh ${ }_{w-\mathrm{fl}}$, the functor $\Xi$, and the triangulated category of relative singularities $\mathrm{D}_{\text {Sing }}^{\prime \prime}\left(X_{0} / X\right)$.

Proof. For each $-n \leq p \leq N$, the restriction of the matrix factorization $\mathcal{M}^{p}$ to the closed subscheme $X_{0} \subset X$ is an unbounded complex of quasicoherent sheaves $i^{*} \mathcal{M}^{p, \bullet}$. By [Polishchuk and Vaintrob 2011, Lemma 1.5], this complex is acyclic.

The complex $\widetilde{\Sigma}^{\prime}\left(\mathcal{M}^{-n}\right) \rightarrow \cdots \rightarrow \widetilde{\Sigma}^{\prime}\left(\mathcal{M}^{N}\right)$ of quasicoherent sheaves on $X_{0}$ is quasi-isomorphic to the total complex of the bicomplex $\mathcal{K}^{\bullet}, \bullet$ with the terms $\mathcal{K}^{p, 0}=i^{*} \mathcal{M}^{p, 0}, \mathcal{K}^{p,-1}=i^{*} \mathcal{M}^{p,-1}, \mathcal{K}^{p,-2}=\operatorname{ker}\left(i^{*} \mathcal{M}^{p,-1} \rightarrow i^{*} \mathcal{M}^{p, 0}\right)$, and $\mathcal{K}^{p, q}=0$ for $q \neq 0,-1,-2$. Similarly, the quasicoherent sheaf $\widetilde{\Sigma}^{\prime}(\mathcal{M})$ on $X_{0}$ is quasi-isomorphic to the total complex of the bicomplex $\mathcal{E}^{\bullet, \bullet}$ with the terms $\mathcal{E}^{p, p}=i^{*} \mathcal{M}^{p, p}, \mathcal{E}^{p, p-1}=i^{*} \mathcal{M}^{p, p-1}, \mathcal{E}^{p, p-2}=\operatorname{ker}\left(i^{*} \mathcal{M}^{p, p-1} \rightarrow i^{*} \mathcal{M}^{p, p}\right)$, and $\mathcal{E}^{p, q}=0$ for $q-p \neq 0,-1,-2$.

We can assume that $N, n \geq 0$. Consider the bicomplex $\mathcal{F}^{\bullet}, \bullet$ with the terms $\mathcal{F}^{p, q}=i^{*} \mathcal{M}^{p, q}$ for $-n-1 \leq q \leq N, \mathcal{F}^{p,-n-2}=\operatorname{ker}\left(i^{*} \mathcal{M}^{p,-n-1} \rightarrow i^{*} \mathcal{M}^{p,-n}\right)$, and $\mathcal{F}^{p, q}=0$ for $q<-n-2$ or $q>N$. Then there are natural surjective morphisms of bicomplexes $\mathcal{F}^{\bullet, \bullet} \rightarrow \mathcal{K}^{\bullet, \bullet}$ and $\mathcal{F}^{\bullet, \bullet} \rightarrow \mathcal{E}^{\bullet} \bullet$. The kernels of both morphisms are the direct sums of a finite bicomplex of quasicoherent sheaves of finite flat dimension on $X_{0}$ and a finite bicomplex of quasicoherent sheaves on $X_{0}$ with acyclic columns. Thus both morphisms become isomorphisms in $\mathrm{D}_{\text {Sing }}^{\prime}\left(X_{0}\right)$.

Remark 3.6. One would like to have a theory of set-theoretic supports for locally free matrix factorizations of finite rank that would allow us to prove Proposition 3.6 in the way similar to the proof of Lemma 3.5. However, we do not know how to do this. In particular, we do not know whether every locally free matrix factorization of finite rank with category-theoretic support in $T$ is isomorphic in the absolute derived category to a direct summand of an object represented by a coherent matrix factorization of finite flat dimension with set-theoretic support in $T$ (cf. Corollary 1.10 and Section 3.3). 
Another alternative approach to proving Proposition 3.6 would be to show that the intersection of the full subcategories $\mathrm{D}^{\text {abs }}((X, \mathcal{L}, w)$-coh $)$ and $\mathrm{D}^{\text {abs }}\left((X, \mathcal{L}, w)\right.$-qcoh If $\left._{\text {If }}\right)$ in the absolute derived category $\mathrm{D}^{\mathrm{abs}}((X, \mathcal{L}, w)$-qcoh) coincides with the full subcategory $\mathrm{D}^{\text {abs }}\left((X, \mathcal{L}, w)-\right.$ coh $\left._{\text {If }}\right)$. We do not know whether this is true.

3.7. Duality and push-forwards. In the following two sections we discuss the compatibility properties of the derived direct and inverse image functors for matrix factorizations with the Serre-Grothendieck duality functors from Section 2.5.

Let $X$ be a separated Noetherian scheme with a dualizing complex $\mathcal{D}_{X}^{\bullet}$, and let $f: Y \rightarrow X$ be a separated morphism of finite type. As usually, we set $\mathcal{D}_{Y}^{\bullet}=f^{+} \mathcal{D}_{X}^{\bullet}$, where $f^{+}$is the functor denoted by $f^{!}$in [Hartshorne 1966] (right adjoint to $\mathbb{R} f_{*}$ for proper morphisms $f$ and left adjoint to $\mathbb{R} f_{*}$ for open embeddings $f$; see [Neeman 1996, Example 4.2] and [Hartshorne 1966, Remark before Proposition V.8.5 and Deligne's Appendix]). This formula defines the dualizing complex $\mathcal{D}_{Y}^{\bullet}$ up to a natural quasi-isomorphism only, and we presume this derived category object (as well as $\mathcal{D}_{X}^{\bullet}$ ) to be represented by a finite complex of injective quasicoherent sheaves.

Proposition 3.7. Let $T \subset Y_{0}$ be a closed subset such that (for some closed subscheme structure on $T$ ) the morphism $\left.f\right|_{T}: T \rightarrow X_{0}$ is proper. Then the derived direct image functor

$$
\overline{\mathbb{R} f_{*}}: \overline{\mathrm{D}_{T}^{\text {abs }}\left(\left(Y, f^{*} \mathcal{L}, f^{*} w\right)-\operatorname{coh}\right)} \longrightarrow \overline{\mathrm{D}^{\text {abs }}((X, \mathcal{L}, w)-\text { coh })}
$$

and the similar functor for the potential $-w$ form a commutative diagram with the Serre duality functors

$$
\begin{gathered}
\mathcal{H o m}_{X \text {-qc }}\left(-, \mathcal{D}_{X}^{\bullet}\right): \mathrm{D}^{\mathrm{abs}}((X, \mathcal{L},-w) \text {-coh })^{\mathrm{op}} \longrightarrow \mathrm{D}^{\mathrm{abs}}((X, \mathcal{L}, w) \text {-coh }), \\
\mathcal{H o m}_{Y \text {-qc }}\left(-, \mathcal{D}_{Y}^{\bullet}\right): \mathrm{D}_{T}^{\mathrm{abs}}\left(\left(Y, f^{*} \mathcal{L},-f^{*} w\right) \text {-coh }\right)^{\mathrm{op}} \longrightarrow \mathrm{D}_{T}^{\mathrm{abs}}\left(\left(Y, f^{*} \mathcal{L}, f^{*} w\right) \text {-coh }\right) .
\end{gathered}
$$

Two proofs of Proposition 3.7 are given below. One of them is based on the theory of set-theoretic supports of coherent CDG-modules developed in Section 1.10 and the arguments similar to the proof of Lemma 3.5. It does not depend on the assumption about $w$ and $f^{*} w$ being local nonzero-divisors and does not mention the zero loci. The other proof is based on the passage to the triangulated categories of relative singularities and uses Proposition 3.5(c).

First proof. First of all, the duality functor

$\mathcal{H}^{o m} m_{Y \text {-qc }}\left(-, \mathcal{D}_{Y}^{\bullet}\right): \mathrm{D}^{\text {abs }}\left(\left(Y, f^{*} \mathcal{L},-f^{*} w\right) \text {-qcoh }\right)^{\mathrm{op}} \longrightarrow \mathrm{D}^{\mathrm{abs}}\left(\left(Y, f^{*} \mathcal{L}, f^{*} w\right)\right.$-qcoh $)$ obviously takes the full subcategory $\mathrm{D}^{\text {abs }}\left(\left(Y, f^{*} \mathcal{L},-f^{*} w\right)-\operatorname{coh}_{T}\right)^{\text {op }}$ into

$$
\mathrm{D}^{\mathrm{abs}}\left(\left(Y, f^{*} \mathcal{L}, f^{*} w\right)-\operatorname{coh}_{T}\right)
$$

and vice versa. Furthermore, for any quasicoherent sheaf $\mathcal{K}$ on $Y$ denote by $\Gamma_{T} \mathcal{K} \subset \mathcal{K}$ the maximal quasicoherent subsheaf with set-theoretic support in $T$. 
Then for any matrix factorization $\mathcal{M} \in \mathrm{D}^{\mathrm{abs}}\left(\left(Y, f^{*} \mathcal{L},-f^{*} w\right)-\operatorname{coh}_{T}\right)$, the natural morphism $\mathcal{H o m}_{Y \text {-qc }}\left(\mathcal{M}, \Gamma_{T} \mathcal{D}_{Y}^{\bullet}\right) \rightarrow \mathcal{H o m}_{Y \text {-qc }}\left(\mathcal{M}, \mathcal{D}_{Y}^{\bullet}\right)$ is an isomorphism in $\mathrm{D}^{\text {abs }}\left(\left(Y, f^{*} \mathcal{L}, f^{*} w\right)-\operatorname{coh}_{T}\right)$.

As in the proof of Lemma 3.5, we will use the construction of the functor

$$
\mathbb{R} f_{*}: \mathrm{D}^{\mathrm{abs}}\left(\left(Y, f^{*} \mathcal{L}, f^{*} w\right) \text {-qcoh }\right) \longrightarrow \mathrm{D}^{\mathrm{abs}}((X, \mathcal{L}, w) \text {-qcoh })
$$

similar to the one from the proof of Proposition 1.9 (see Remarks 1.8 and 1.9). Let $\left\{U_{\alpha}\right\}$ and $\left\{V_{\beta}\right\}$ be two affine open coverings of the scheme $Y$. For any matrix factorization $\mathcal{N} \in\left(Y, f^{*} \mathcal{L},-f^{*} w\right)$-qcoh, there is a natural morphism of bicomplexes of matrix factorizations

$$
f_{*} C_{\left\{U_{\alpha}\right\}}^{\bullet} \mathcal{H o m}_{Y \text {-qc }}\left(\mathcal{N}, \Gamma_{T} \mathcal{D}_{Y}^{\bullet}\right) \longrightarrow \mathcal{H o m}_{X \text {-qc }}\left(f_{*} \mathcal{N}, f_{*} C_{\left\{U_{\alpha}\right\}}^{\bullet} \Gamma_{T} \mathcal{D}_{Y}^{\bullet}\right)
$$

Passing to the total complexes and taking the composition with the adjunction morphism $\left.f_{*} C_{\left\{U_{\alpha}\right\}}\right\}^{\bullet} \Gamma_{T} \mathcal{D}_{Y}^{\bullet}=\mathbb{R} f_{*}\left(\Gamma_{T} \mathcal{D}_{Y}^{\bullet}\right) \rightarrow \mathcal{D}_{X}^{\bullet}$, we obtain a natural morphism of complexes of matrix factorizations

$$
f_{*} C_{\left\{U_{\alpha}\right\}}^{\bullet} \mathcal{H o m}_{Y-\mathrm{qc}}\left(\mathcal{N}, \Gamma_{T} \mathcal{D}_{Y}^{\bullet}\right) \longrightarrow \mathcal{H o m}_{X \text {-qc }}\left(f_{*} \mathcal{N}, \mathcal{D}_{X}^{\bullet}\right)
$$

(cf. [Neeman 1996, beginning of Section 6]).

Substituting $\mathcal{N}=C_{\left\{V_{\beta}\right\}} \mathcal{M}$ for some $\mathcal{M} \in\left(Y, f^{*} \mathcal{L},-f^{*} w\right)$-qcoh, we get a natural morphism of bicomplexes of matrix factorizations

$$
f_{*} C_{\left\{U_{\alpha}\right\}}^{\bullet} \mathcal{H o m}_{Y-\mathrm{qc}}\left(C_{\left\{V_{\beta}\right\}}^{\bullet} \mathcal{M}, \Gamma_{T} \mathcal{D}_{Y}^{\bullet}\right) \longrightarrow \mathcal{H o m}_{X \text {-qc }}\left(f_{*} C_{\left\{V_{\beta}\right\}}^{\bullet} \mathcal{M}, \mathcal{D}_{X}^{\bullet}\right)
$$

When $\mathcal{M}$ is a coherent matrix factorization supported set-theoretically in $T$, the induced morphism of the total complexes is a quasi-isomorphism of complexes of matrix factorizations by the conventional Serre-Grothendieck duality theorem for bounded derived categories of coherent sheaves and proper morphisms of schemes (see [Hartshorne 1966, Theorem VII.3.3] or [Neeman 1996, Section 6]). Hence the induced morphism of the total matrix factorizations is an isomorphism in $\mathrm{D}^{\text {abs }}((X, \mathcal{L}, w)$-qcoh $)$, and consequently also in $\mathrm{D}^{\text {abs }}((X, \mathcal{L}, w)$-coh).

Second proof. Assume that $w$ and $f^{*} w$ are locally nonzero-dividing sections of the respective line bundles. Let $i: X_{0} \rightarrow X$ be the zero locus of $w$ and $i^{\prime}: Y_{0} \rightarrow Y$ be the zero locus of $f^{*} w$. As above, we set $\mathcal{D}_{X_{0}}^{\bullet}=\mathbb{R} i^{!} \mathcal{D}_{X}^{\bullet}$ and $\mathcal{D}_{Y_{0}}^{\bullet}=\mathbb{R} i^{\prime !} \mathcal{D}_{Y}^{\bullet}$ [Hartshorne 1966, Proposition V.2.4], and presume all these dualizing complexes to be finite complexes of injective quasicoherent sheaves.

The duality functor

$$
\mathcal{H} m_{Y \text {-qc }}\left(-, \mathcal{D}_{Y}^{\bullet}\right): \mathrm{D}^{\mathrm{abs}}\left(\left(Y, f^{*} \mathcal{L},-f^{*} w\right) \text {-coh }\right)^{\mathrm{op}} \longrightarrow \mathrm{D}^{\mathrm{abs}}\left(\left(Y, f^{*} \mathcal{L}, f^{*} w\right) \text {-coh }\right)
$$

is compatible with the restrictions to the open subscheme $Y \backslash T$ and thus identifies the full subcategories $\mathrm{D}_{T}^{\mathrm{abs}}\left(\left(Y, f^{*} \mathcal{L},-f^{*} w\right) \text {-coh }\right)^{\mathrm{op}}$ and $\mathrm{D}_{T}^{\mathrm{abs}}\left(\left(Y, f^{*} \mathcal{L}, f^{*} w\right)\right.$-coh $)$. To prove the proposition, we will define the Serre duality functors on the triangulated 
categories of relative singularities $\mathrm{D}_{\text {Sing }}^{\mathrm{b}}\left(Y_{0} / Y\right)$ and $\mathrm{D}_{\text {Sing }}^{\mathrm{b}}\left(X / X_{0}\right)$, then check that the equivalences of triangulated categories $\mathbb{E} \Xi=\Upsilon^{-1}$ commute with the dualities, and finally reduce to the conventional Serre-Grothendieck duality theorem for bounded complexes of coherent sheaves.

The duality functor $\mathcal{H o m}_{X_{0} \text {-qc }}\left(-, \mathcal{D}_{X_{0}}^{\bullet}\right): \mathrm{D}^{\mathrm{b}}\left(X_{0} \text {-coh }\right)^{\mathrm{op}} \rightarrow \mathrm{D}^{\mathrm{b}}\left(X_{0}\right.$-coh $)$ takes objects of the form $\mathbb{L} i^{*} \mathcal{K}^{\bullet}$, where $\mathcal{K}^{\bullet} \in \mathrm{D}^{\mathrm{b}}(X$-coh $)$, to similar objects. Indeed, one has

$$
\mathcal{H o m}_{X_{0} \text {-qc }}\left(\mathbb{L} i^{*} \mathcal{K}^{\bullet}, \mathcal{D}_{X_{0}}^{\bullet}\right) \simeq \mathbb{R} i^{!} \mathcal{H} \operatorname{Hom}_{X \text {-qc }}\left(\mathcal{K}^{\bullet}, \mathcal{D}_{X}^{\bullet}\right)
$$

[loc. cit., Proposition V.8.5] and $\left.\mathbb{R} i ! \simeq \mathcal{L}\right|_{X_{0}}[-1] \otimes_{\mathcal{O}_{X_{0}}} \mathbb{L} i^{*}$ (see the proof of Theorem 2.7). Therefore, we have the induced duality functor

$$
\mathcal{H}_{\text {om }} X_{0^{-q c}}\left(-, \mathcal{D}_{X_{0}}^{\bullet}\right): \mathrm{D}_{\text {Sing }}^{\mathrm{b}}\left(X_{0} / X\right)^{\mathrm{op}} \longrightarrow \mathrm{D}_{\text {Sing }}^{\mathrm{b}}\left(X_{0} / X\right) .
$$

Similarly, the duality functor

$$
\mathcal{H} \operatorname{Hom}_{Y_{0} \text {-qc }}\left(-, \mathcal{D}_{Y_{0}}^{\bullet}\right): \mathrm{D}^{\mathrm{b}}\left(Y_{0^{-}} \text {coh }\right)^{\mathrm{op}} \longrightarrow \mathrm{D}^{\mathrm{b}}\left(Y_{0} \text {-coh }\right)
$$

takes the full subcategory $\mathrm{D}^{\mathrm{b}}\left(Y_{0}-\operatorname{coh}_{T}\right)^{\mathrm{op}}$ into $\mathrm{D}^{\mathrm{b}}\left(Y_{0}\right.$-coh $\left._{T}\right)$ and $\operatorname{Perf}_{T}\left(Y_{0} / Y\right)^{\mathrm{op}}$ into $\operatorname{Perf}_{T}\left(Y_{0} / Y\right)$. Hence the induced duality functor

$$
\mathcal{H}_{\text {om }_{Y_{0}-\mathrm{qc}}}\left(-, \mathcal{D}_{Y_{0}}^{\bullet}\right): \mathrm{D}_{\text {Sing }}^{\mathrm{b}}\left(Y_{0} / Y, T\right)^{\mathrm{op}} \longrightarrow \mathrm{D}_{\text {Sing }}^{\mathrm{b}}\left(Y_{0} / Y, T\right) .
$$

Checking that the equivalence of categories $\mathrm{D}^{\text {abs }}((X, \mathcal{L}, w)$-coh $) \simeq \mathrm{D}_{\text {Sing }}^{\mathrm{b}}\left(X_{0} / X\right)$ commutes with the dualities is easily done using the functor $\Upsilon$. It suffices to notice the functorial quasi-isomorphism $\mathcal{H o m}_{X \text {-qc }}\left(i_{*} \mathcal{F}^{\bullet}, \mathcal{D}_{X}^{\bullet}\right) \simeq i_{*} \mathcal{H o m}_{X_{0} \text {-qc }}\left(\mathcal{F}^{\bullet}, \mathcal{D}_{X_{0}}^{\bullet}\right)$ for any complex $\mathcal{F}^{\bullet} \in \mathrm{D}^{\mathrm{b}}\left(X_{0}\right.$-coh) [loc. cit., Theorem III.6.7]. The same applies to the equivalence of categories

$$
\overline{\mathrm{D}_{T}^{\text {abs }}\left(\left(Y, f^{*} \mathcal{L}, f^{*} w\right)-\operatorname{coh}\right)} \simeq \overline{\mathrm{D}_{\text {Sing }}^{\mathrm{b}}\left(Y_{0} / Y, T\right)} .
$$

Furthermore, by Proposition 3.5(c), the equivalences of categories $\mathbb{\Xi}=\Upsilon^{-1}$ transform the derived direct image functor

$$
\overline{\mathbb{R} f_{*}}: \overline{\mathrm{D}_{T}^{\text {abs }}\left(\left(Y, f^{*} \mathcal{L}, f^{*} w\right)-\operatorname{coh}\right)} \longrightarrow \overline{\mathrm{D}^{\text {abs }}((X, \mathcal{L}, w)-\operatorname{coh})}
$$

into (the idempotent closure of) the direct image functor $f_{\circ}: \mathrm{D}_{\text {Sing }}^{\mathrm{b}}\left(Y_{0} / Y, T\right) \rightarrow$ $\mathrm{D}_{\text {Sing }}^{\mathrm{b}}\left(X_{0} / X\right)$.

Finally, the direct image functor $f_{\circ}: \mathrm{D}_{\text {Sing }}^{\mathrm{b}}\left(Y_{0} / Y, T\right) \rightarrow \mathrm{D}_{\text {Sing }}^{\mathrm{b}}\left(X_{0} / X\right)$ commutes with the Serre duality functors since so do the derived direct image functors $\left.\mathbb{R} f\right|_{T *}: \mathrm{D}^{\mathrm{b}}(\tilde{T}$-coh $) \rightarrow \mathrm{D}^{\mathrm{b}}\left(X_{0}\right.$-coh $)$ for all the closed subscheme structures $\tilde{T} \subset Y_{0}$ on the closed subset $T$ and the similar functors related to the closed embeddings $\widetilde{T}^{\prime} \rightarrow \widetilde{T}^{\prime \prime}$ of various such subscheme structures into each other. This is the conventional Serre-Grothendieck duality theorem for proper morphisms of schemes. 
3.8. Duality and pull-backs. Let $X$ be a separated Noetherian scheme with a dualizing complex $\mathcal{D}_{X}^{\bullet}$ and $f: Y \rightarrow X$ be a separated morphism of finite type; set $\mathcal{D}_{Y}^{\bullet}=f^{+} \mathcal{D}_{X}^{\bullet}$. Let $\mathcal{L}$ be a line bundle on $X$ and $w \in \mathcal{L}(X)$ be a section.

Let us first suppose that the morphism $f$ is smooth of relative dimension $n$. Then the functor $f^{+}: \mathrm{D}^{+}(X$-qcoh $) \rightarrow \mathrm{D}^{+}(Y$-qcoh $)$ is naturally isomorphic to $\omega_{Y / X}[n] \otimes_{\mathcal{O}_{Y}} f^{*}$, where $\omega_{Y / X}$ is the line bundle of relative top forms.

In particular, $\mathcal{D}_{Y}^{\bullet} \simeq \omega_{Y / X}[n] \otimes_{\mathcal{O}_{Y}} f^{*} \mathcal{D}_{X}^{\bullet}$ (where $f^{*} \mathcal{D}_{X}^{\bullet}$ is also presumed to have been replaced by a complex of injectives). Then it is clear that the equivalences of categories

$$
\begin{aligned}
& \mathcal{D}_{X}^{\bullet} \otimes_{\mathcal{O}_{X}}-: \mathrm{D}^{\mathrm{co}}\left((X, \mathcal{L}, w)-\mathrm{qcoh}_{\mathrm{fl}}\right) \longrightarrow \mathrm{D}^{\mathrm{co}}((X, \mathcal{L}, w) \text {-qcoh }), \\
& f^{*} \mathcal{D}_{X}^{\bullet} \otimes_{\mathcal{O}_{Y}}-: \mathrm{D}^{\mathrm{co}}\left(\left(Y, f^{*} \mathcal{L}, f^{*} w\right)-\mathrm{qcoh}{ }_{\mathrm{fl}}\right) \longrightarrow \mathrm{D}^{\mathrm{co}}\left(\left(Y, f^{*} \mathcal{L}, f^{*} w\right) \text {-qcoh }\right)
\end{aligned}
$$

from Section 2.5 transform the inverse image functor for flat matrix factorizations $f^{*}: \mathrm{D}^{\mathrm{co}}\left((X, \mathcal{L}, w)\right.$-qcoh $\left.{ }_{\mathrm{fl}}\right) \rightarrow \mathrm{D}^{\mathrm{co}}\left(\left(Y, f^{*} \mathcal{L}, f^{*} w\right)\right.$-qcoh $\left.{ }_{\mathrm{fl}}\right)$ into the (underived, as the morphism $f$ is flat) inverse image functor for quasicoherent matrix factorizations $f^{*}: \mathrm{D}^{\mathrm{co}}((X, \mathcal{L}, w)$-qcoh $) \rightarrow \mathrm{D}^{\mathrm{co}}\left(\left(Y, f^{*} \mathcal{L}, f^{*} w\right)\right.$-qcoh $)$.

Furthermore, for any quasicoherent matrix factorization $\mathcal{M}$ on $X$ there is a natural morphism of finite complexes of matrix factorizations $f^{*} \mathcal{H}$ om $_{X \text {-qc }}\left(\mathcal{M}, \mathcal{D}_{X}^{\bullet}\right) \rightarrow$ $\mathcal{H o m}_{Y \text {-qc }}\left(f^{*} \mathcal{M}, f^{*} \mathcal{D}_{X}^{\bullet}\right)$ on $Y$. When $\mathcal{M}$ is a coherent matrix factorization, this is a quasi-isomorphism of complexes of matrix factorizations (since the similar assertion holds for coherent sheaves [Hartshorne 1966, Proposition II.5.8]), so the related morphism of total matrix factorizations has an absolutely acyclic cone. Thus the antiequivalences of categories

$$
\mathcal{H} m_{X \text {-qc }}\left(-, \mathcal{D}_{X}^{\bullet}\right): \mathrm{D}^{\text {abs }}((X, \mathcal{L},-w) \text {-coh })^{\mathrm{op}} \longrightarrow \mathrm{D}^{\mathrm{abs}}((X, \mathcal{L}, w) \text {-coh }),
$$

$\mathcal{H o m}_{Y \text {-qc }}\left(-, f^{*} \mathcal{D}_{X}^{\bullet}\right): \mathrm{D}^{\mathrm{abs}}\left(\left(Y, f^{*} \mathcal{L},-f^{*} w\right) \text {-coh }\right)^{\mathrm{op}} \longrightarrow \mathrm{D}^{\mathrm{abs}}\left(\left(Y, f^{*} \mathcal{L}, f^{*} w\right)\right.$-coh $)$

form a commutative diagram with the inverse image functors $f^{*}$ for coherent matrix factorizations.

Now suppose that $f$ is a proper morphism of finite type. The following theorem describes the compatibility property of the covariant Serre-Grothendieck duality with the inverse images of matrix factorizations (cf. [Positselski 2012, Theorem 5.15.3], where a similar result is proven for complexes of quasicoherent sheaves).

Theorem 3.8. The equivalences of categories

$$
\begin{gathered}
\mathcal{D}_{X}^{\bullet} \otimes_{\mathcal{O}_{X}}-: \mathrm{D}^{\mathrm{abs}}\left((X, \mathcal{L}, w)-\text { qcoh }_{\mathrm{fl}}\right) \longrightarrow \mathrm{D}^{\mathrm{co}}((X, \mathcal{L}, w)-\mathrm{qcoh}), \\
\mathcal{D}_{Y}^{\bullet} \otimes_{\mathcal{O}_{Y}}-: \mathrm{D}^{\mathrm{abs}}\left(\left(Y, f^{*} \mathcal{L}, f^{*} w\right)-\mathrm{qcoh}_{\mathrm{fl}}\right) \longrightarrow \mathrm{D}^{\mathrm{co}}\left(\left(Y, f^{*} \mathcal{L}, f^{*} w\right) \text {-qcoh }\right)
\end{gathered}
$$

transform the inverse image functor

$$
f^{*}: \mathrm{D}^{\mathrm{abs}}\left((X, \mathcal{L}, w)-\mathrm{qcoh}_{\mathrm{fl}}\right) \longrightarrow \mathrm{D}^{\mathrm{abs}}\left(\left(Y, f^{*} \mathcal{L}, f^{*} w\right)-\mathrm{qcoh}_{\mathrm{fl}}\right)
$$


into the functor $f^{!}: \mathrm{D}^{\mathrm{co}}((X, \mathcal{L}, w)$-qcoh $) \rightarrow \mathrm{D}^{\mathrm{co}}\left(\left(Y, f^{*} \mathcal{L}, f^{*} w\right)\right.$-qcoh $)$ right adjoint to the direct image functor

$$
\mathbb{R} f_{*}: \mathrm{D}^{\mathrm{co}}\left(\left(Y, f^{*} \mathcal{L}, f^{*} w\right) \text {-qcoh }\right) \longrightarrow \mathrm{D}^{\mathrm{co}}((X, \mathcal{L}, w) \text {-qcoh })
$$

(see the end of Section 1.8).

Proof. For any quasicoherent matrix factorization $\mathcal{N}$ on $Y$ and any flat quasicoherent matrix factorization $\mathcal{E}$ on $X$, we have to construct an isomorphism

$$
\begin{aligned}
\operatorname{Hom}_{D^{c o}((X, \mathcal{L}, w) \text {-qcoh })}\left(\mathbb{R} f_{*} \mathcal{N}, \mathcal{D}_{X}^{\bullet} \otimes_{\mathcal{O}_{X}} \mathcal{E}\right) \\
\simeq \operatorname{Hom}_{D^{c o}\left(\left(Y, f^{*} \mathcal{L}, f^{*} w\right) \text {-qcoh }\right)}\left(\mathcal{N}, \mathcal{D}_{Y}^{\bullet} \otimes_{\mathcal{O}_{Y}} f^{*} \mathcal{E}\right) .
\end{aligned}
$$

The composition

$$
\begin{aligned}
\operatorname{Hom}_{Y}\left(\mathcal{N}, \mathcal{D}_{Y}^{\bullet}\right. & \left.\otimes_{\mathcal{O}_{Y}} f^{*} \mathcal{E}\right) \longrightarrow \operatorname{Hom}_{X}\left(\mathbb{R} f_{*} \mathcal{N}, \mathbb{R} f_{*}\left(\mathcal{D}_{Y}^{\bullet} \otimes_{\mathcal{O}_{Y}} f^{*} \mathcal{E}\right)\right) \\
& \simeq \operatorname{Hom}_{X}\left(\mathbb{R} f_{*} \mathcal{N}, f_{*} \mathcal{D}_{Y}^{\bullet} \otimes_{\mathcal{O}_{X}} \mathcal{E}\right) \longrightarrow \operatorname{Hom}_{X}\left(\mathbb{R} f_{*} \mathcal{N}, \mathcal{D}_{X}^{\bullet} \otimes_{\mathcal{O}_{X}} \mathcal{E}\right)
\end{aligned}
$$

provides a morphism from the right-hand to the left-hand side. Here all the Hom functors are taken in the coderived categories of quasicoherent matrix factorizations on $Y$ and $X$; the middle isomorphism holds since $\mathcal{D}_{Y}^{\bullet} \otimes_{\mathcal{O}_{Y}} f^{*} \mathcal{E}$ is an injective matrix factorization on $Y$ (so the derived direct image can be computed for it by applying the underived direct image functor $f_{*}$ termwise) and by the projection formula; the last morphism is induced by the adjunction $f_{*} \mathcal{D}_{Y}^{\bullet} \rightarrow \mathcal{D}_{X}^{\bullet}$.

Furthermore, on both sides of the desired isomorphism we have injective matrix factorizations in the second arguments of the Hom functors; hence the Hom can be computed in the homotopy category of matrix factorizations instead of the coderived category in both cases. Finally, one can assume $\mathcal{N}$ to be an injective matrix factorization, too, and compute $\mathbb{R} f_{*} \mathcal{N}=f_{*} \mathcal{N}$ termwise (alternatively, one could use the Čech construction). Similarly, the tensor products in the second arguments are totalizations of termwise tensor products.

Now one can fix the components involved for both matrix factorizations $\mathcal{N}$ and $\mathcal{E}$, obtaining a morphism of finite complexes of abelian groups of the same kind as above, but related to (one-term) complexes of quasicoherent sheaves rather than matrix factorizations. The latter is an isomorphism by [Positselski 2012, Theorem 5.15.3]. It remains to notice that the totalization of an acyclic finite complex of (unbounded) complexes of abelian groups is acyclic.

The next corollary is a matrix factorization version of the main result of Deligne's appendix to [Hartshorne 1966] (see also [Positselski 2012, Section 5.16]).

Corollary 3.8. For any morphism of finite type between separated Noetherian schemes with dualizing complexes $f: Y \rightarrow X$, a line bundle $\mathcal{L}$ on $X$, and a section 
$w \in \mathcal{L}(X)$, one can define a triangulated functor

$$
\left.f^{+}: \mathrm{D}^{\mathrm{co}}((X, \mathcal{L}, w) \text {-qcoh }) \longrightarrow \mathrm{D}^{\mathrm{co}}\left(\left(Y, f^{*} \mathcal{L}, f^{*} w\right)\right) \text {-qcoh }\right)
$$

in such a way that

(i) for an open embedding $f$, one has $f^{+}=f^{*}$, and more generally, for a smooth morphism $f$ of relative dimension $n$ one has $f^{+}=\omega_{Y / X}[n] \otimes_{\mathcal{O}_{Y}} f^{*}$;

(ii) for a proper morphism $f$, the functor $f^{+}=f^{!}$is right adjoint to $\mathbb{R} f_{*}$;

(iii) the construction is compatible with the compositions of the morphisms $f$.

Proof. It suffices to define $f^{+}: \mathrm{D}^{\mathrm{co}}((X, \mathcal{L}, w)$-qcoh $) \rightarrow \mathrm{D}^{\mathrm{co}}\left(\left(Y, f^{*} \mathcal{L}, f^{*} w\right)\right.$-qcoh $)$ as the functor corresponding to the inverse image of flat quasicoherent matrix factorizations $f^{*}: \mathrm{D}^{\text {abs }}\left((X, \mathcal{L}, w)\right.$-qcoh $\left.{ }_{\mathrm{fl}}\right) \rightarrow \mathrm{D}^{\text {abs }}\left(\left(Y, f^{*} \mathcal{L}, f^{*} w\right)\right.$-qcoh fl $\left._{\mathrm{fl}}\right)$ under the identifications of categories

$$
\begin{aligned}
\mathcal{D}_{X}^{\bullet} \otimes_{\mathcal{O}_{X}}-: \mathrm{D}^{\mathrm{abs}}\left((X, \mathcal{L}, w)-\mathrm{qcoh}_{\mathrm{fl}}\right) & \longrightarrow \mathrm{D}^{\mathrm{co}}((X, \mathcal{L}, w) \text {-qcoh }), \\
\mathcal{D}_{Y}^{\bullet} \otimes_{\mathcal{O}_{Y}}-: \mathrm{D}^{\mathrm{abs}}\left(\left(Y, f^{*} \mathcal{L}, f^{*} w\right)-\text { qcoh }_{\mathrm{fl}}\right) & \longrightarrow \mathrm{D}^{\mathrm{co}}\left(\left(Y, f^{*} \mathcal{L}, f^{*} w\right) \text {-qcoh }\right),
\end{aligned}
$$

where $\mathcal{D}_{X}^{\bullet}$ is any dualizing complex on $X$ and $\mathcal{D}_{Y}^{\bullet}=f^{+} \mathcal{D}_{X}^{\bullet}$.

\section{Appendix A. Quasicoherent graded modules}

A.1. Flat quasicoherent sheaves. I am grateful to A. Neeman for suggesting that a result of the following kind can be proven without much difficulty.

Lemma A.1. On any quasicompact semiseparated scheme, any quasicoherent sheaf is the quotient sheaf of a flat quasicoherent sheaf.

Proof. Let $X$ be our scheme. Assume that a quasicoherent sheaf $\mathcal{M}$ over $X$ is flat over an open subscheme $V \subset X$; given an affine open subscheme $U \subset X$, we will construct a surjective morphism $\mathcal{N} \rightarrow \mathcal{M}$ onto $\mathcal{M}$ from a quasicoherent sheaf $\mathcal{N}$ over $X$ that is flat over $U \cup V$. Let $j$ denote the embedding $U \rightarrow X$. There exists a surjective morphism onto $j^{*} \mathcal{M}$ from a flat quasicoherent sheaf $\mathcal{F}$ over $U$; let $\mathcal{K}$ denote the kernel of this morphism of sheaves.

Since the morphism $j: U \rightarrow X$ is affine and flat, the functor $j_{*}$ is exact and preserves flatness. Consider the pull-back of the exact triple $j_{*} \mathcal{K} \rightarrow j_{*} \mathcal{F} \rightarrow j_{*} j^{*} \mathcal{M}$ with respect to the morphism $\mathcal{M} \rightarrow j_{*} j^{*} \mathcal{M}$; denote the middle term of the resulting exact triple by $\mathcal{N}$. One has $\left.\mathcal{N}\right|_{U}=\left.\mathcal{F}\right|_{U}$, so $\mathcal{N}$ is flat over $U$. Furthermore, the sheaf $j^{*} \mathcal{M}$ is flat over $V \cap U$; hence, so is the sheaf $\mathcal{K}$. The embedding $U \cap V \rightarrow V$ is an affine flat morphism, so the sheaf $j_{*} \mathcal{K}$ is flat over $V$. From the exact triple $j_{*} \mathcal{K} \rightarrow \mathcal{N} \rightarrow \mathcal{M}$, we conclude that $\mathcal{N}$ is flat over $V$.

It follows immediately that any quasicoherent graded module over a quasicoherent graded algebra $\mathcal{B}$ over $X$ is a quotient module of a flat quasicoherent graded module. 
A.2. Locally projective quasicoherent graded modules. The following result is essentially due to Raynaud and Gruson [1971] (for a discussion, see [Drinfeld 2006, Section 2]); here we just briefly explain how to deduce the formulation that interests us from their assertions.

Theorem A.2. Let $X$ be an affine scheme and $\left\{U_{\alpha}\right\}$ be its finite affine covering. Let $\mathcal{B}$ be a quasicoherent graded algebra over $X$ and $\mathcal{P}$ be a quasicoherent graded module over $\mathcal{B}$. Then the graded $\mathcal{B}(X)$-module $\mathcal{P}(X)$ is projective if and only if the graded $\mathcal{B}\left(U_{\alpha}\right)$-module $\mathcal{P}\left(U_{\alpha}\right)$ is projective for every $\alpha$.

Proof. First of all, a graded module $P$ over a graded ring $B$ is projective if and only if it is projective as an ungraded module. Indeed, if $P$ is graded projective, then it is a homogeneous direct summand of a free graded module; hence $P$ is also ungraded projective. Conversely, pick a homogeneous (of degree 0 ) surjective homomorphism $F \rightarrow P$ onto a given graded module $P$ from a free graded module $F$. If $P$ is ungraded projective, this homomorphism has a (perhaps nonhomogeneous) section $s$, and the homogeneous component of $s$ of degree 0 provides a homogeneous section. Hence it suffices to consider ungraded modules over an ungraded quasicoherent algebra $\mathcal{B}$.

It is clear that if $\mathcal{P}(X)$ is a projective $\mathcal{B}(X)$-module, then $\mathcal{P}(V)$ is a projective $\mathcal{B}(V)$-module for any affine open subscheme $V \subset X$. Conversely, assume that the $\mathcal{B}\left(U_{\alpha}\right)$-module $\mathcal{P}\left(U_{\alpha}\right)$ is projective for every $\alpha$. Then by the result of [Kaplansky 1958], the $\mathcal{B}\left(U_{\alpha}\right)$-modules $\mathcal{P}\left(U_{\alpha}\right)$ are direct sums of countably generated modules, and it follows easily that so is the $\mathcal{B}(X)$-module $\mathcal{P}(X)$ (essentially, since a connected graph with an at most countable set of edges at each vertex has a countable number of vertices). Hence we can assume the $\mathcal{B}(X)$-module $\mathcal{P}(X)$ to be countably generated.

Besides, the $\mathcal{B}\left(U_{\alpha}\right)$-modules $\mathcal{P}\left(U_{\alpha}\right)$ are flat; hence so is the $\mathcal{B}(X)$-module $\mathcal{P}(X)$. By [Raynaud and Gruson 1971, Corollaire II.2.2.2], it remains to show that the $\mathcal{B}(X)$-module $\mathcal{P}(X)$ satisfies the Mittag-Leffler condition; this can be easily deduced from the similar property of the $\mathcal{B}\left(U_{\alpha}\right)$-modules $\mathcal{P}\left(U_{\alpha}\right)$ using the formulation of this condition given in Proposition II.2.1.4(iii) or Propositions II.2.1.4(ii) and II.2.1.1(i) of [Raynaud and Gruson 1971] (cf. Sections II.2.5 and II.3.1 of the same paper).

A.3. Injective quasicoherent graded modules. The following result is a noncommutative generalization of a theorem of Hartshorne [1966, Theorem II.7.18] about injective quasicoherent sheaves on Noetherian schemes. Our proof method, based on the Artin-Rees lemma, is different from the one in [loc. cit.].

Theorem A.3. Let $\mathcal{B}$ be a Noetherian quasicoherent graded algebra over a Noetherian scheme $X$. Then any injective object in the category of quasicoherent graded left modules over $\mathcal{B}$ is also an injective object of the category of arbitrary sheaves of graded $\mathcal{B}$-modules over $X$. 
Consequently, the restriction $\left.\mathcal{J}\right|_{U}$ of an injective quasicoherent graded module $\mathcal{J}$ over $\mathcal{B}$ to an open subscheme $U \subset X$ is an injective quasicoherent graded module over $\left.\mathcal{B}\right|_{U}$. Conversely, if $U_{\alpha}$ is an open covering of $X$ and the quasicoherent graded $\left.\mathcal{B}\right|_{U_{\alpha}}$-modules $\left.\mathcal{J}\right|_{U_{\alpha}}$ are injective, then a quasicoherent graded $\mathcal{B}$-module $\mathcal{J}$ is injective. Besides, the underlying sheaf of graded abelian groups of any injective quasicoherent graded $\mathcal{B}$-module $\mathcal{J}$ is flabby.

Proof. First of all, notice that the abelian category $\mathcal{B}$-qcoh of quasicoherent graded modules over $\mathcal{B}$ is a locally Noetherian Grothendieck category with coherent graded modules forming the subcategory of Noetherian generators [Hartshorne 1977, Exercise II.5.15]; so, in particular, $\mathcal{B}$-qcoh has enough injectives and the assertions of Theorem A.3 are not vacuous. The category of sheaves of graded $\mathcal{B}$-modules $\mathcal{B}$-mod has similar properties, with the extensions by zero of the restrictions of $\mathcal{B}$ to (small) open subschemes of $X$ forming a set of Noetherian generators [Hartshorne 1966, Theorem II.7.8].

Secondly, let us check that the main result in the first paragraph implies the assertions in the second one. Indeed, injective sheaves of graded $\mathcal{B}$-modules have all the properties we are interested in. They remain injective after being restricted to an open subscheme since the extension by zero from an open subscheme is an exact functor. They are flabby since given two open subschemes $U \subset V \subset X$ and $j_{U}, j_{V}$ being their identity embeddings $U, V \rightarrow X$, the morphism of sheaves of graded $\mathcal{B}$-modules $\left.\left.j_{U !} \mathcal{B}\right|_{U} \rightarrow j_{V !} \mathcal{B}\right|_{V}$ is injective. And their property is local [loc. cit., Lemma II.7.16] because sheaves of graded $\mathcal{B}$-modules supported inside one of the subschemes $U_{\alpha}$ form a set of generators of the category $\mathcal{B}$-mod.

Now let $\mathcal{J}$ be an injective quasicoherent graded module over $\mathcal{B}$. To prove the main assertion, we have to show that for any open subscheme $U \subset X$ and a subsheaf of graded $\mathcal{B}$-modules $\left.\mathcal{G} \subset j_{U} ! \mathcal{B}\right|_{U}$, any homogeneous morphism of sheaves of graded $\mathcal{B}$-modules $\mathcal{G} \rightarrow \mathcal{J}$ can be extended to a similar morphism $\left.j_{U !} \mathcal{B}\right|_{U} \rightarrow \mathcal{J}$. Indeed, $\mathcal{G}$ is a subsheaf of graded $\mathcal{B}$-modules in the coherent graded $\mathcal{B}$-module $\mathcal{B}$; hence according to the following proposition, there exists a quasicoherent graded $\mathcal{B}$-module $\mathcal{G} \subset \mathcal{F} \subset \mathcal{B}$ such that the morphism $\mathcal{G} \rightarrow \mathcal{J}$ can be extended to a homogeneous morphism of quasicoherent graded $\mathcal{B}$-modules $\mathcal{F} \rightarrow \mathcal{J}$.

Since $\mathcal{J}$ is injective in $\mathcal{B}$-qcoh, the latter morphism can in turn be extended to a similar morphism $\mathcal{B} \rightarrow \mathcal{J}$. Restricting to $\left.j_{U !} \mathcal{B}\right|_{U}$, we obtain the desired morphism of sheaves of graded $\mathcal{B}$-modules $\left.j_{U} ! \mathcal{B}\right|_{U} \rightarrow \mathcal{J}$.

Proposition A.3. In the assumptions of Theorem A.3, let $\mathcal{E}$ be a coherent graded left $\mathcal{B}$-module, $\mathcal{G} \subset \mathcal{E}$ be a subsheaf of graded $\mathcal{B}$-modules, $\mathcal{M}$ be a quasicoherent graded $\mathcal{B}$-module, and $\phi: \mathcal{G} \rightarrow \mathcal{M}$ be a morphism of sheaves of graded $\mathcal{B}$-modules. Then there exists a coherent graded $\mathcal{B}$-module $\mathcal{G} \subset \mathcal{F} \subset \mathcal{E}$ such that the morphism $\phi$ can be extended to $\mathcal{F}$. 
Proof. Before proving Proposition A.3, let us reformulate its conclusion as follows. In the same setting, there exists a quasicoherent graded $\mathcal{B}$-module $\mathcal{K}$ together with an injective morphism $\mathcal{M} \rightarrow \mathcal{K}$ and a morphism $\mathcal{E} \rightarrow \mathcal{K}$ forming a commutative diagram with the embedding $\mathcal{G} \rightarrow \mathcal{E}$ and the morphism $\phi: \mathcal{G} \rightarrow \mathcal{M}$. Indeed, if a coherent $\mathcal{B}$-module $\mathcal{F}$ exists, one can take $\mathcal{K}$ to be the fibered coproduct of $\mathcal{E}$ and $\mathcal{M}$ over $\mathcal{F}$; conversely, if a quasicoherent $\mathcal{B}$-module $\mathcal{K}$ exists, one can take $\mathcal{F}$ to be the full preimage of $\mathcal{M} \subset \mathcal{K}$ under the morphism $\mathcal{E} \rightarrow \mathcal{K}$. Notice also that one can always replace $\mathcal{M}$ with its sufficiently big coherent graded $\mathcal{B}$-submodule.

Now let us state the version of Artin-Rees lemma that we will use.

Lemma A.3. In the assumptions of Theorem A.3, let $\mathcal{M}$ be a coherent graded $\mathcal{B}$-module, $\mathcal{N} \subset \mathcal{M}$ a coherent graded $\mathcal{B}$-submodule, and $Z \subset X$ a closed subscheme with the sheaf of ideals $\mathcal{I}_{Z} \subset \mathcal{O}_{X}$. Then for any $n \geq 0$, there exists $m \geq 0$ such that the intersection $\mathcal{I}_{Z}^{m} \mathcal{M} \cap \mathcal{N}$ is contained in $\mathcal{I}_{Z}^{n} \mathcal{N}$.

Proof. Clearly, the question is local, so it suffices to consider the case of an affine scheme $X$. Then (the graded version of) the Artin-Rees lemma for ideals generated by central elements in noncommutative Noetherian rings [Goodearl and Warfield 1989, Theorem 13.3] applies.

Being a Noetherian object, the sheaf of graded $\mathcal{B}$-modules $\mathcal{G}$ is generated by a finite number of homogeneous sections $s_{n} \in \mathcal{G}\left(U_{n}\right)$, where $U_{n} \subset X$ are some open subschemes. If all of these subschemes coincide with $X$, the sheaf $\mathcal{G}$, being a subsheaf of a coherent sheaf generated by global sections, is itself coherent, so there is nothing to prove. In the general case, we will argue by induction on the number of open subschemes $U_{n}$ that are not equal to $X$.

Let $U=U_{1} \subsetneq X$ be one such open subscheme, and $T=X \backslash U$ be its closed complement. We can assume that $\mathcal{M}$ is a coherent graded $\mathcal{B}$-module. Let $\mathcal{N}$ denote its maximal coherent graded $\mathcal{B}$-submodule supported set-theoretically in $T$. Applying Lemma A.3 to $\mathcal{N} \subset \mathcal{M}$, we conclude that there is a closed subscheme structure $i: Z \rightarrow X$ on $T$ such that the morphism $\mathcal{N} \rightarrow i_{*} i^{*} \mathcal{M}$ is injective. Consequently, so is the morphism $\mathcal{M} \rightarrow i_{*} i^{*} \mathcal{M} \oplus j_{*} j^{*} \mathcal{M}$, where $j$ denotes the open embedding $U \rightarrow X$.

Let us show that there is a thicker closed subscheme structure $i^{\prime}: Z^{\prime} \rightarrow X$ on $T$ such that the kernel of the morphism of sheaves $i_{*}^{\prime} i^{\prime *} \mathcal{G} \rightarrow i_{*}^{\prime} i^{\prime *} \mathcal{E}$ is contained in the kernel of the morphism of sheaves $i_{*}^{\prime} i^{\prime *} \mathcal{G} \rightarrow i_{*} i^{*} \mathcal{G}$. Indeed, there exists a finite collection of subsheaves of graded $\mathcal{B}$-modules in $\mathcal{G}$, each of them an extension by zero of a coherent graded $\left.\mathcal{B}\right|_{V}$-module from some open subscheme $V \subset X$ such that the stalk of $\mathcal{G}$ at each point of $X$ coincides with the stalk of one of these subsheaves. So the assertion reduces to the case when $\mathcal{G}$ is a coherent graded $\mathcal{B}$-submodule in $\mathcal{E}$ when it is an equivalent reformulation of Lemma A.3. 
Let $\mathcal{H} \subset i^{\prime *} \mathcal{E}$ denote the image of the morphism of sheaves of graded $i^{\prime *} \mathcal{B}$-modules $i^{\prime *} \mathcal{G} \rightarrow i^{*} \mathcal{E}$ over the scheme $Z^{\prime}$. Let $\iota: Z \rightarrow Z^{\prime}$ be the natural closed embedding. Then, according to the above, the morphism of sheaves of graded $i^{\prime *} \mathcal{B}$-modules $i^{\prime *} \mathcal{G} \rightarrow \iota_{*} i^{*} \mathcal{G}$ induces a morphism $\mathcal{H} \rightarrow \iota_{*} i^{*} \mathcal{G}$.

The sheaf of graded $i^{\prime *} \mathcal{B}$-modules $\mathcal{H}$ is generated by the images of the restrictions of the sections $s_{n}, n \geq 2$, to the closed subschemes $Z^{\prime} \cap U_{n} \subset U_{n}$. Hence the induction assumption is applicable to $\mathcal{H}$, and we can conclude that there exists a quasicoherent graded $i^{\prime *} \mathcal{B}$-module $\mathcal{K}$ on the scheme $Z^{\prime}$ together with an injective morphism $\iota_{*} i^{*} \mathcal{M} \rightarrow \mathcal{K}$ and a morphism $i^{\prime *} \mathcal{E} \rightarrow \mathcal{K}$ forming a commutative diagram with the embedding $\mathcal{H} \rightarrow i^{\prime *} \mathcal{E}$ and the composition $\mathcal{H} \rightarrow \iota_{*} i^{*} \mathcal{G} \rightarrow \iota_{*} i^{*} \mathcal{M}$.

Similarly, the sheaf of graded $\left.\mathcal{B}\right|_{U}$-modules $j^{*} \mathcal{G}$ is generated by the restrictions of the sections $s_{n}$ to the open subschemes $U_{1} \cap U_{n} \subset U_{n}$, among which the (restriction of) the section $s_{1}$ is a global section over $U=U_{1}$. Hence the induction assumption is applicable to $j^{*} \mathcal{G}$, and there exists a quasicoherent graded $\left.\mathcal{B}\right|_{U}$-module $\mathcal{L}$ together with an injective morphism $j^{*} \mathcal{M} \rightarrow \mathcal{L}$ and a morphism $j^{*} \mathcal{E} \rightarrow \mathcal{L}$ forming a commutative diagram with the embedding $j^{*} \mathcal{G} \rightarrow j^{*} \mathcal{E}$ and the morphism $j^{*} \mathcal{G} \rightarrow j^{*} \mathcal{M}$.

Now the injective morphism $\mathcal{M} \rightarrow i_{*}^{\prime} \mathcal{K} \oplus j_{*} \mathcal{L}$ (whose first component is the composition $\left.\mathcal{M} \rightarrow i_{*} i^{*} \mathcal{M} \simeq i_{*}^{\prime} \iota_{*} i^{*} \mathcal{M} \rightarrow i_{*}^{\prime} \mathcal{K}\right)$ and the morphism $\mathcal{E} \rightarrow i_{*}^{\prime} \mathcal{K} \oplus j_{*} \mathcal{L}$ provide the desired commutative diagram of morphisms of sheaves of graded $\mathcal{B}$-modules over $X$.

\section{Appendix B. Hochschild (co)homology of matrix factorizations}

This appendix complements the paper [Polishchuk and Positselski 2012] in two ways. Section B.1 contains some modifications and improvements of the main results of [loc. cit.] generally, and as applied to locally free matrix factorizations of finite rank in particular. The main thrust consists of replacing the finite homological dimension conditions in [loc. cit.] with the Noetherianness conditions to the (limited) extent possible.

Section B.2, on the other hand, presents an elementary approach to the computation of Hochschild (co)homology of coherent matrix factorizations, entirely unrelated to that in [loc. cit.] and not based on any notion of Hochschild (co)homology of the second kind, but rather on the Serre-Grothendieck duality theory.

B.1. Locally free matrix factorizations of finite rank. In Sections B.1.1-B.1.4, we start with a bit of categorical nonsense, following the lines of [Polishchuk and Positselski 2012, Sections 3.3-3.5], but with the additional coherence/Noetherianness conditions imposed from the very beginning. We use the notation from [loc. cit.] rather than that of the main body of this paper. Then in Section B.1.5, we turn to locally free matrix factorizations of finite rank over certain possibly singular, affine algebraic varieties. Finally, Section B.1.6 presents an improvement over 
the discussion of matrix factorizations over smooth affine varieties in [loc. cit., Section 4.8]. An example of an application of our techniques to nonaffine varieties can be found in the preprint [Efimov 2012].

B.1.1. Coherent and Noetherian $C D G$-categories. Let $(\Gamma, \sigma, \mathbf{1})$ be a grading group data [Polishchuk and Positselski 2012, Section 1.1] and $B^{\#}$ be a small $\Gamma$-graded preadditive category [Positselski 2011a, Section A.1]. Both left and right $\Gamma$-graded $B^{\#}$-modules form abelian categories.

A $\Gamma$-graded $B^{\#}$-module is said to be finitely generated (respectively, finitely presented) if it is a quotient module of a finitely generated free $\Gamma$-graded $B^{\#}$-module [Polishchuk and Positselski 2012, Section 1.5] (respectively, the cokernel of a morphism of finitely generated free $\Gamma$-graded $B^{\#}$-modules).

A $\Gamma$-graded preadditive category $B^{\#}$ is called left Noetherian if any submodule of a finitely generated $\Gamma$-graded left $B^{\#}$-module is finitely generated, or equivalently, if the abelian category of $\Gamma$-graded left $B^{\#}$-modules is locally Noetherian. A $\Gamma$-graded preadditive category $B^{\#}$ is called left coherent if any submodule of a finitely presented $\Gamma$-graded left $B^{\#}$-module is finitely presented.

Let $B$ be a small ( $\Gamma$-graded) CDG-category [loc. cit., Section 1.2] and $B^{\#}$ be its underlying $\Gamma$-graded preadditive category. Following [loc. cit.], we denote the DG-categories of left and right CDG-modules over $B$ by $B$-mod ${ }^{\text {cdg }}$ and $\bmod ^{\mathrm{cdg}}-B$. The DG-subcategories of left CDG-modules whose underlying $\Gamma$-graded $B^{\#}$-modules are flat or injective are denoted by $B-\bmod _{\mathrm{fl}}^{\text {cdg }}$ and $B-\bmod _{\mathrm{inj}}^{\text {cdg }} C$ $B$-mod ${ }^{\text {cdg }}$. Similarly, the DG-subcategories of left and right CDG-modules over $B$ whose underlying $\Gamma$-graded $B^{\#}$-modules are projective and finitely generated are denoted by $B-\bmod _{\mathrm{fgp}}^{\mathrm{cdg}}$ and $\bmod _{\mathrm{fgp}}^{\mathrm{cdg}}-B$.

Assuming that the $\Gamma$-graded category $B^{\#}$ is left Noetherian, the DG-subcategory of left CDG-modules whose underlying $\Gamma$-graded $B^{\#}$-modules are finitely generated is denoted by $B$-mod fg $_{\mathrm{fg}}^{\mathrm{cdg}} \subset B$-mod ${ }^{\text {cdg }}$. Assuming that the $\Gamma$-graded category $B^{\#}$ is right coherent, the DG-subcategory of right CDG-modules whose underlying $\Gamma$-graded $B^{\#}$-modules are finitely presented is denoted by $\bmod _{\mathrm{fp}}^{\mathrm{cdg}}-B$.

The coderived and contraderived categories of left CDG-modules over $B$ are denoted by $\mathrm{D}^{\mathrm{co}}\left(B-\mathrm{mod}^{\mathrm{cdg}}\right)$ and $\mathrm{D}^{\mathrm{ctr}}\left(B-\right.$ mod $\left.^{\mathrm{cdg}}\right)$, respectively [loc. cit., Section 3.2]. Assuming that the $\Gamma$-graded category $B^{\#}$ is right coherent, the class of flat $\Gamma$-graded left $B$-modules [loc. cit., Section 2.2] is closed under infinite products, so the contraderived category $\mathrm{D}^{\mathrm{ctr}}\left(B-\bmod _{\mathrm{fl}}^{\mathrm{cdg}}\right)$ is well-defined. The homotopy category of the DG-category $B$-mod ${ }_{\mathrm{inj}}^{\mathrm{cdg}}$ is denoted, as usually, by $H^{0}\left(B-\bmod _{\mathrm{inj}}^{\mathrm{cdg}}\right)$.

In the respective assumptions of left Noetherianness or right coherence of the $\Gamma$-graded category $B^{\#}$, the absolute derived categories of CDG-modules with finitely generated or finitely presented underlying $\Gamma$-graded $B^{\#}$-modules are denoted by $\mathrm{D}^{\mathrm{abs}}\left(B-\mathrm{mod}_{\mathrm{fg}}^{\mathrm{cdg}}\right)$ and $\mathrm{D}^{\mathrm{abs}}\left(\bmod _{\mathrm{fp}}^{\mathrm{cdg}}-B\right)$, respectively. 
B.1.2. Derived functors of the second kind. Let $k$ be a commutative ring and $B$ be a small $k$-linear CDG-category. Assume that the $\Gamma$-graded category $B^{\#}$ is left Noetherian. Let $L$ and $M$ be left CDG-modules over $B$; suppose that the $\Gamma$-graded left $B^{\#}$-module $L^{\#}$ underlying the CDG-module $L$ over $B$ is finitely generated.

As in [Polishchuk and Positselski 2012, §§ 2.1-2], we denote by $Z^{0}\left(B-\bmod ^{\text {cdg }}\right)$ and $Z^{0}\left(\bmod ^{\mathrm{cdg}}-B\right)$ the abelian categories of left and right CDG-modules over $B$. Let $Z^{0}\left(B-\bmod _{\mathrm{fg}}^{\mathrm{cdg}}\right) \subset Z^{0}\left(B-\bmod ^{\mathrm{cdg}}\right)$ and $H^{0}\left(B-\bmod _{\mathrm{fg}}^{\mathrm{cdg}}\right) \subset H^{0}\left(B-\bmod ^{\text {cdg }}\right)$ denote the abelian and homotopy categories of left CDG-modules over $B$ with finitely generated underlying $\Gamma$-graded $B^{\#}$-modules, and $Z^{0}\left(\bmod _{\mathrm{fp}}^{\mathrm{cdg}}-B\right) \subset Z^{0}\left(\bmod ^{\mathrm{cdg}}-B\right)$ and $H^{0}\left(\bmod _{\mathrm{fp}}^{\mathrm{cdg}}-B\right) \subset H^{0}\left(\bmod ^{\mathrm{cdg}}-B\right)$ be the similar categories of right CDG-modules with finitely presented underlying $\Gamma$-graded modules.

Let $J^{\bullet}$ be a right resolution of $M$ in $Z^{0}\left(B\right.$-mod $\left.{ }^{\text {cdg }}\right)$ such that the $\Gamma$-graded left $B^{\#}$-modules $J^{i \#}$ are injective, and let $J$ be the total CDG-module of the complex of CDG-modules $J^{\bullet}$ constructed by taking infinite direct sums along the diagonals. Then the complex $\operatorname{Tot}^{\oplus} \operatorname{Hom}^{B}\left(L, J^{\bullet}\right)$ computing $\operatorname{Ext}_{B}^{I I}(L, M)$ [loc. cit., Section 2.2] is isomorphic to the complex $\operatorname{Hom}^{B}(L, J)$ [loc. cit., formula (6)], which computes the $k$-modules of morphisms from $L$ into $M[*]$ in the coderived category $\mathrm{D}^{\mathrm{co}}\left(B\right.$-mod $\left.{ }^{\mathrm{cdg}}\right)$ [Positselski 2011b, Theorems 3.5(a) and 3.7]. Thus,

$$
H^{*} \operatorname{Ext}_{B}^{I I}(L, M) \simeq \operatorname{Hom}_{\mathrm{D}^{\mathrm{co}}\left(B-\bmod ^{\mathrm{cdg}}\right)}(L, M[*]) .
$$

Just as in [Polishchuk and Positselski 2012, Section 3.3], one can lift this isomorphism from the level of cohomology modules to that of the derived category $\mathrm{D}(k$-mod $)$ in the following way. Consider the functor

$$
\operatorname{Hom}^{B}: H^{0}\left(B-\bmod ^{\text {cdg }}\right)^{\text {op }} \times H^{0}\left(B-\bmod ^{\text {cdg }}\right) \longrightarrow \mathrm{D}(k-\bmod ),
$$

and restrict it to the full subcategory $H^{0}\left(B-\bmod _{\mathrm{inj}}^{\mathrm{cdg}}\right)$ in the second argument. This restriction factorizes through the coderived category $\mathrm{D}^{\mathrm{co}}\left(B\right.$-mod $\left.{ }^{\mathrm{cdg}}\right)$ in the first argument. Taking into account [Positselski 2011b, Theorem 3.7], we obtain a right derived functor

$$
\mathrm{D}^{\mathrm{co}}\left(B-\bmod ^{\mathrm{cdg}}\right)^{\mathrm{op}} \times \mathrm{D}^{\mathrm{co}}\left(B-\bmod ^{\mathrm{cdg}}\right) \longrightarrow \mathrm{D}(k-\bmod ) .
$$

Restricting to the full subcategory $\mathrm{D}^{\mathrm{abs}}\left(B-\bmod _{\mathrm{fg}}^{\mathrm{cdg}}\right)^{\mathrm{op}} \subset \mathrm{D}^{\mathrm{co}}\left(B-\bmod ^{\mathrm{cdg}}\right)^{\mathrm{op}}$ [loc. cit., Theorem 3.11.1] in the first argument, we have the derived functor

$$
\mathrm{D}^{\mathrm{abs}}\left(B-\bmod _{\mathrm{fg}}^{\mathrm{cdg}}\right)^{\mathrm{op}} \times \mathrm{D}^{\mathrm{co}}\left(B-\bmod ^{\mathrm{cdg}}\right) \longrightarrow \mathrm{D}(k-\bmod ) .
$$

The composition of this functor with the localization functors

$$
Z^{0}\left(B-\bmod _{\mathrm{fg}}^{\mathrm{cdg}}\right) \longrightarrow \mathrm{D}^{\mathrm{abs}}\left(B-\bmod _{\mathrm{fg}}^{\mathrm{cdg}}\right) \quad \text { and } \quad Z^{0}\left(B-\bmod ^{\mathrm{cdg}}\right) \longrightarrow \mathrm{D}^{\mathrm{co}}\left(B-\bmod ^{\mathrm{cdg}}\right)
$$

agrees with the derived functor $\operatorname{Ext}_{B}^{I I}$ where the former is defined. 
Now assume that the $\Gamma$-graded category $B^{\#}$ is right coherent. Consider the functor [Polishchuk and Positselski 2012, formula (5)]

$$
\otimes_{B}: H^{0}\left(\bmod ^{\mathrm{cdg}}-B\right) \times H^{0}\left(B-\bmod ^{\mathrm{cdg}}\right) \longrightarrow \mathrm{D}(k-\bmod )
$$

and restrict it to the Cartesian product of full subcategories

$$
H^{0}\left(\bmod _{\mathrm{fp}}^{\mathrm{cdg}}-B\right) \times H^{0}\left(B-\bmod _{\mathrm{fl}}^{\mathrm{cdg}}\right) \subset H^{0}\left(\bmod ^{\mathrm{cdg}}-B\right) \times H^{0}\left(B-\bmod ^{\mathrm{cdg}}\right) .
$$

Since the tensor product with a finitely presented $\Gamma$-graded right $B^{\#}$-module commutes with infinite products of $\Gamma$-graded left $B^{\#}$-modules, this restriction factorizes through the contraderived category $\mathrm{D}^{\mathrm{ctr}}\left(B-\bmod _{\mathrm{fl}}^{\mathrm{cdg}}\right)$ in the second argument. Clearly, it also factorizes through the absolute derived category $\mathrm{D}^{\mathrm{abs}}\left(\bmod _{\mathrm{fp}}^{\mathrm{cdg}}-B\right)$ in the first argument.

By Remark 1.5 of the main body of this paper (see also [Positselski 2012, Proposition A.3.1(b)]), the natural functor $\mathrm{D}^{\mathrm{ctr}}\left(B-\bmod _{\mathrm{fl}}^{\mathrm{cdg}}\right) \rightarrow \mathrm{D}^{\mathrm{ctr}}\left(B-\bmod ^{\mathrm{cdg}}\right)$ is an equivalence of triangulated categories. Hence we obtain the left derived functor

$$
\mathrm{D}^{\mathrm{abs}}\left(\bmod _{\mathrm{fp}}^{\mathrm{cdg}}-B\right) \times \mathrm{D}^{\mathrm{ctr}}\left(B-\bmod ^{\mathrm{cdg}}\right) \longrightarrow \mathrm{D}(k-\bmod ) .
$$

Up to composing with the localization functors $Z^{0}\left(\bmod _{\mathrm{fp}}^{\mathrm{cdg}}-B\right) \rightarrow \mathrm{D}^{\mathrm{abs}}\left(\bmod _{\mathrm{fp}}^{\mathrm{cdg}}-B\right)$ and $Z^{0}\left(B-\bmod ^{\text {cdg }}\right) \rightarrow \mathrm{D}^{\text {ctr }}\left(B\right.$-mod $\left.{ }^{\text {cdg }}\right)$, this functor agrees with the derived functor $\operatorname{Tor}^{B, I I}$ from [Polishchuk and Positselski 2012, Section 2.2] where the former is defined.

Indeed, let $N$ be an object of $Z^{0}\left(\bmod _{\mathrm{fp}}^{\mathrm{cdg}}-B\right)$. Let $P$. be a left resolution of an object $M \in Z^{0}\left(B\right.$-mod $\left.{ }^{\text {cdg }}\right)$ by left CDG-modules over $B$ with flat underlying $\Gamma$-graded $B^{\#}$-modules, and let $P$ be the total CDG-module of the complex $P$. constructed by taking infinite products along the diagonals. Then the complex $\operatorname{Tot}^{\Pi}\left(N \otimes_{B} P_{\bullet}\right)$ computing $\operatorname{Tor}^{B, I I}(N, M)$ is isomorphic to the complex $N \otimes_{B} P$ computing the derived functor (5) on the objects $N$ and $M$.

B.1.3. Comparison of the two theories. Let $C$ be a small $k$-linear ( $\Gamma$-graded) DG-category. The above constructions applicable to CDG-categories and CDG-modules over them can be also applied to DG-categories and DG-modules as a particular case. Following [Polishchuk and Positselski 2012], we denote the DG-categories of left and right DG-modules over $C$ by $C$-mod ${ }^{\mathrm{dg}}$ and $\bmod ^{\mathrm{dg}}-C$, and generally use the upper index "dg" instead of "cdg" in the notation related to DG-modules.

As in [loc. cit., Sections $2.1,3.1$ and 3.4], we denote by $H^{0}\left(C-\text { mod }^{\mathrm{dg}}\right)_{\text {inj }}$ and $H^{0}\left(C \text {-mod }{ }^{\mathrm{dg}}\right)_{\mathrm{fl}}$ the homotopy categories of h-injective and h-flat left DG-modules over $C$. The notation $H^{0}\left(C-\bmod _{\text {inj }}^{\mathrm{dg}}\right)_{\text {inj }}$ and $H^{0}\left(C-\bmod _{\mathrm{fl}}^{\mathrm{dg}}\right)_{\mathrm{fl}}$ stands for the full triangulated subcategories in $H^{0}\left(C\right.$-mod $\left.{ }^{\mathrm{dg}}\right)$ formed by h-injective DG-modules over $C$ whose underlying $\Gamma$-graded $C^{\#}$-modules are injective, or h-flat DG-modules whose underlying $\Gamma$-graded $C^{\#}$-modules are flat, respectively. Finally, we let 
$H^{0}\left(C-\bmod _{\mathrm{fgp}}^{\mathrm{dg}}\right)_{\mathrm{prj}} \subset H^{0}\left(C-\bmod ^{\mathrm{dg}}\right)$ and $H^{0}\left(\bmod _{\mathrm{fgp}}^{\mathrm{dg}}-C\right)_{\mathrm{fl}} \subset H^{0}\left(\bmod ^{\mathrm{dg}}-C\right)$ denote the full triangulated subcategories of h-projective left and h-flat right DG-modules whose underlying $\Gamma$-graded $C^{\#}$-modules are projective and finitely generated.

Assume that the $\Gamma$-graded category $C^{\#}$ is left Noetherian. Let $L$ be an object of $Z^{0}\left(C-\bmod _{\mathrm{fg}}^{\mathrm{dg}}\right)$. Given a left DG-module $M$ over $C$, pick its injective resolution $J^{\bullet}$ in the exact category $Z^{0}\left(C\right.$-mod $\left.{ }^{\mathrm{dg}}\right)$ [loc. cit., Section 2.1]. Let $\operatorname{Tot}^{\oplus}\left(J^{\bullet}\right) \rightarrow$ $\operatorname{Tot}^{\square}\left(J^{\bullet}\right)$ be the natural closed morphism between the total DG-modules of the complex $J^{\bullet}$ constructed by taking infinite direct sums and infinite products along the diagonals. Then the induced morphism of complexes of $k$-modules

$$
\operatorname{Hom}^{C}\left(L, \operatorname{Tot}^{\oplus}\left(J^{\bullet}\right)\right) \longrightarrow \operatorname{Hom}^{C}\left(L, \operatorname{Tot}^{\sqcap}\left(J^{\bullet}\right)\right)
$$

represents the comparison morphism $\operatorname{Ext}_{C}^{I I}(L, M) \rightarrow \operatorname{Ext}_{C}(L, M)$ [loc. cit., formula (10)] in $\mathrm{D}(k$-mod) between the two kinds of Ext objects for the DG-modules $L$ and $M$.

Similarly, assume that the $\Gamma$-graded category $C^{\#}$ is right coherent. Let $N$ be an object of $Z^{0}\left(\bmod _{\mathrm{fp}}^{\mathrm{dg}}-C\right)$. Given a left DG-module $M$ over $C$, pick its projective resolution $P_{\bullet}$ in the exact category $Z^{0}\left(C\right.$-mod $\left.{ }^{\mathrm{dg}}\right)$. Let $\operatorname{Tot}^{\oplus}\left(P_{\bullet}\right) \rightarrow \operatorname{Tot}^{\square}\left(P_{\bullet}\right)$ be the natural closed morphism between the total DG-modules of the complex $P$. constructed by taking infinite direct sums and infinite products along the diagonals. Then the induced morphism of complexes of $k$-modules

$$
N \otimes_{C} \operatorname{Tot}^{\oplus}\left(P_{\bullet}\right) \longrightarrow N \otimes_{C} \operatorname{Tot}^{\sqcap}\left(P_{\bullet}\right)
$$

represents the comparison morphism $\operatorname{Tor}^{C}(N, M) \rightarrow \operatorname{Tor}^{C, I I}(N, M)$ [loc. cit., formula (9)] in $\mathrm{D}(k$-mod) between the two kinds of Tor objects for the DG-modules $N$ and $M$.

Proposition A. Assume that the $\Gamma$-graded category $C^{\#}$ is left Noetherian. Let $L$ be a left DG-module over $C$ whose underlying $\Gamma$-graded left $C^{\#}$-module is finitely generated, and let $M$ be a left DG-module over $C$. Then the natural morphism $\operatorname{Ext}_{C}^{I I}(L, M) \rightarrow \operatorname{Ext}_{C}(L, M)$ is an isomorphism provided that either

(i) the object $M \in \mathrm{D}^{\mathrm{co}}\left(C\right.$-mod $\left.{ }^{\mathrm{dg}}\right)$ belongs to the image of the fully faithful functor $H^{0}\left(C-\bmod _{\text {inj }}^{\mathrm{dg}}\right)_{\mathrm{inj}} \rightarrow \mathrm{D}^{\mathrm{co}}\left(C-\bmod ^{\mathrm{dg}}\right) ;$ or

(ii) the object $L \in \mathrm{D}^{\mathrm{abs}}\left(C\right.$-mod $\left.{ }^{\mathrm{dg}}\right)$ belongs to the image of the fully faithful functor $H^{0}\left(C-\bmod _{\mathrm{fgp}}^{\mathrm{dg}}\right)_{\mathrm{prj}} \rightarrow \mathrm{D}^{\mathrm{abs}}\left(C-\bmod _{\mathrm{fg}}^{\mathrm{dg}}\right)$.

Proof. Let $J^{\bullet}$ be an injective resolution of the DG-module $M$ in the exact category $Z^{0}\left(C\right.$-mod $\left.{ }^{\mathrm{dg}}\right)$. Then the natural morphism $M \rightarrow \operatorname{Tot}^{\oplus}\left(J^{\bullet}\right)$ is always an isomorphism in $\mathrm{D}^{\mathrm{co}}\left(C\right.$-mod $\left.{ }^{\mathrm{dg}}\right)$ [Positselski 2011b, proof of Theorem 3.7], while the morphism $M \rightarrow \operatorname{Tot}^{\square}\left(J^{\bullet}\right)$ is an isomorphism in the conventional derived category $\mathrm{D}\left(C-\bmod ^{\mathrm{dg}}\right)$ [loc. cit., proofs of Theorems 1.4-5]. Furthermore, one has $\operatorname{Tot}^{\oplus}\left(J^{\bullet}\right) \in H^{0}\left(C-\bmod _{\text {inj }}^{\mathrm{dg}}\right)$ and $\operatorname{Tot}^{\sqcap}\left(J^{\bullet}\right) \in H^{0}\left(C-\bmod _{\text {inj }}^{\mathrm{dg}}\right)_{\text {inj }}$. 
Part (i): The functor is fully faithful by [loc. cit., Theorem 3.5(a) and Lemma 1.3]. According to formula (4) from Section B.1.2 and [Polishchuk and Positselski 2012, Section 3.1], both kinds of Ext involved are well-defined as functors of the argument $M \in \mathrm{D}^{\mathrm{co}}\left(C\right.$-mod $\left.{ }^{\mathrm{dg}}\right)$. Hence one can assume $M \in H^{0}\left(C \text { - } \bmod _{\mathrm{inj}}^{\mathrm{dg}}\right)_{\mathrm{inj}}$. Then both morphisms $M \rightarrow \operatorname{Tot}^{\oplus}\left(J^{\bullet}\right)$ and $M \rightarrow \operatorname{Tot}^{\Pi}\left(J^{\bullet}\right)$ are homotopy equivalences by semiorthogonality; hence so is the morphism $\operatorname{Tot}^{\oplus}\left(J^{\bullet}\right) \rightarrow \operatorname{Tot}^{\square}\left(J^{\bullet}\right)$ and the assertion follows.

Part (ii): In view of the first paragraph of this proof, a cone $K$ of the morphism $\operatorname{Tot}^{\oplus}\left(J^{\bullet}\right) \rightarrow \operatorname{Tot}^{\sqcap}\left(J^{\bullet}\right)$ in $H^{0}\left(C\right.$-mod $\left.{ }^{\text {dg }}\right)$ is an acyclic DG-module over $C$ whose underlying $\Gamma$-graded $C^{\#}$-module is injective. Hence the complex of morphisms $\operatorname{Hom}^{C}(-, K)$ is a well-defined functor $\mathrm{D}^{\mathrm{abs}}\left(C-\bmod _{\mathrm{fg}}^{\mathrm{dg}}\right)^{\mathrm{op}} \rightarrow \mathrm{D}(k$-mod $)$ annihilating $H^{0}\left(C-\bmod _{\mathrm{fgp}}^{\mathrm{dg}}\right)_{\mathrm{prj}}$.

Proposition B. Assume that the $\Gamma$-graded category $C^{\#}$ is right coherent. Let $N$ be a right DG-module over $C$ whose underlying $\Gamma$-graded right $C^{\#-m o d u l e ~ i s ~ f i n i t e l y ~}$ presented, and let $M$ be a left DG-module over $C$. Then the natural morphism $\operatorname{Tor}^{C}(N, M) \rightarrow \operatorname{Tor}^{C, I I}(N, M)$ is an isomorphism provided that either

(i) there exists a closed morphism $P \rightarrow M$ into $M$ from a $D G$-module $P \in$ $H^{0}\left(C-\bmod _{\mathrm{fl}}^{\mathrm{dg}}\right)_{\mathrm{fl}}$ with a cone contraacyclic with respect to $C-\bmod ^{\mathrm{dg}}$ or completely acyclic with respect to $C-\bmod _{\mathrm{fl}}^{\mathrm{dg}}$ (see [Polishchuk and Positselski 2012, Sections 3.2 and 4.7]); or

(ii) the object $N \in \mathrm{D}^{\mathrm{abs}}$ (mod $\operatorname{mo}_{-} \mathrm{C}$ ) belongs to the image of the fully faithful functor $H^{0}\left(\bmod _{\mathrm{fgp}}^{\mathrm{dg}}-C\right)_{\mathrm{fl}} \rightarrow \mathrm{D}^{\mathrm{abs}}\left(\bmod _{\mathrm{fp}}^{\mathrm{dg}}-C\right)$.

Proof. Let $P_{\bullet}$ be a projective resolution of the DG-module $M$ in the exact category $Z^{0}\left(C\right.$-mod $\left.{ }^{\mathrm{dg}}\right)$. Then the natural morphism $\operatorname{Tot}^{\square}\left(P_{\bullet}\right) \rightarrow M$ is always an isomorphism in $\mathrm{D}^{\mathrm{ctr}}\left(C-\right.$ mod $\left.^{\mathrm{dg}}\right)$ [Positselski 2011b, proof of Theorem 3.8], while the morphism $\operatorname{Tot}^{\oplus}\left(P_{\bullet}\right) \rightarrow M$ is an isomorphism in $\mathrm{D}\left(C\right.$-mod $\left.{ }^{\mathrm{dg}}\right)$ [loc. cit., proof of Theorem 1.4]. Furthermore, one has $\operatorname{Tot}^{\sqcap}\left(P_{\bullet}\right) \in H^{0}\left(C-\bmod _{\mathrm{fl}}^{\mathrm{dg}}\right)$ and $\operatorname{Tot}^{\oplus}\left(P_{\bullet}\right) \in H^{0}\left(C-\bmod _{\mathrm{fl}}^{\mathrm{dg}}\right)_{\mathrm{fl}}$.

Part (i): Acyclic DG-modules in the second argument are annihilated by the functor Tor ${ }^{C}$ by [Polishchuk and Positselski 2012, Section 3.1], while contraacyclic DG-modules in the second argument are annihilated by the functor Tor ${ }^{C, I I}(N,-)$ according to the formula (5). The latter also applies to DG-modules completely acyclic with respect to $C$ - $\bmod _{\mathrm{fl}}^{\mathrm{dg}}$ since the functor of a tensor product with a finitely presented DG-module preserves infinite direct sums and products. So one can replace $M$ with $P$ and assume that $M \in H^{0}\left(C-\bmod _{\mathrm{fl}}^{\mathrm{dg}}\right)_{\mathrm{fl}}$.

Then a cone of the morphism $\operatorname{Tot}^{\Pi}\left(P_{\bullet}\right) \rightarrow M$ is contraacyclic with respect to $C$-mod ${ }^{\mathrm{dg}}$ with a flat underlying $\Gamma$-graded $C^{\#}$-module, and hence also contraacyclic 
with respect to $C$ - $\bmod _{\mathrm{fl}}^{\mathrm{dg}}$. On the other hand, a cone of the morphism $\operatorname{Tot}^{\oplus}\left(P_{\bullet}\right) \rightarrow M$ is acyclic and h-flat. It follows that the functor $N \otimes_{C}-$ transforms both of these morphisms, and therefore also the morphism $\operatorname{Tot}^{\oplus}\left(P_{\bullet}\right) \rightarrow \operatorname{Tot}^{\square}\left(P_{\bullet}\right)$, into quasiisomorphisms of complexes of $k$-modules.

Part (ii): A cone $K$ of the morphism $\operatorname{Tot}^{\oplus}\left(P_{\bullet}\right) \rightarrow \operatorname{Tot}^{\sqcap}\left(P_{\bullet}\right)$ in $H^{0}\left(C\right.$-mod $\left.{ }^{\mathrm{dg}}\right)$ is an acyclic DG-module over $C$ whose underlying $\Gamma$-graded $C^{\#}$-module is flat. Hence the tensor product $-\otimes_{C} K$ is a well-defined functor $\mathrm{D}^{\mathrm{abs}}\left(\bmod _{\mathrm{fp}}^{\mathrm{dg}}-C\right) \rightarrow \mathrm{D}(k-\mathrm{mod})$ annihilating $H^{0}\left(\bmod _{\mathrm{fgp}}^{\mathrm{dg}}-C\right)_{\mathrm{fl}}$.

In particular, assuming that the category $C^{\#}$ is left Noetherian, the natural morphism $\operatorname{Ext}_{C}^{I I}(L, M) \rightarrow \operatorname{Ext}_{C}(L, M)$ is an isomorphism for all $L \in C-\bmod _{\mathrm{fg}}^{\mathrm{dg}}$ and $M \in C$-mod ${ }^{\mathrm{dg}}$ provided that the Verdier localization functor $\mathrm{D}^{\mathrm{co}}\left(C\right.$-mod $\left.{ }^{\mathrm{dg}}\right) \rightarrow$ $\mathrm{D}\left(C\right.$-mod $\left.{ }^{\mathrm{dg}}\right)$ is an equivalence of triangulated categories. Assuming that the category $C^{\#}$ is right coherent, the natural morphism $\operatorname{Tor}^{C}(N, M) \rightarrow \operatorname{Tor}^{C, I I}(N, M)$ is an isomorphism for all $N \in \bmod _{\mathrm{fp}}^{\mathrm{dg}}-C$ and $M \in C$-mod ${ }^{\mathrm{dg}}$ provided that the Verdier localization functor $\mathrm{D}^{\mathrm{ctr}}\left(C\right.$-mod $\left.{ }^{\mathrm{dg}}\right) \rightarrow \mathrm{D}\left(C-\right.$ mod $\left.^{\mathrm{dg}}\right)$ is an equivalence of categories, or alternatively, that any acyclic DG-module from $C-\bmod _{\mathrm{fl}}^{\mathrm{dg}}$ is completely acyclic with respect to $C-\bmod _{\mathrm{fl}}^{\mathrm{dg}}$.

B.1.4. Comparison for the DG-category of $C D G$-modules. Let $B$ be a $k$-linear CDG-category and $C=\bmod _{\mathrm{fgp}}^{\mathrm{cdg}}-B$ be the DG-category of right CDG-modules over $B$ whose underlying $\Gamma$-graded $B^{\#}$-modules are projective and finitely generated. The DG-categories of (left or right) CDG-modules over $B$ and DG-modules over $C$ are naturally equivalent [Polishchuk and Positselski 2012, Sections 1.5 and 2.6] (as are the categories of $\Gamma$-graded modules over $B^{\#}$ and $C^{\#}$ ).

Following [loc. cit., Section 3.5], we denote by $M_{C}$ the DG-module over $C$ corresponding to a CDG-module $M$ over $B$.

Let $k^{\vee}$ be an injective cogenerator of the abelian category of $k$-modules. Introduce the notation $B$-mod ${ }_{\mathrm{prj}}^{\mathrm{cdg}} \subset B$-mod ${ }^{\text {cdg }}$ for the DG-category of left CDG-modules over $B$ with projective underlying $\Gamma$-graded $B^{\#}$-modules. The results below in this section are to be compared with those from [loc. cit., Sections 3.5 and 4.7].

Proposition $\mathbf{A}^{\prime}$. Assume that the $\Gamma$-graded category $B^{\#}$ is left Noetherian. Let $L$ be a left $C D G$-module over $B$ whose underlying $\Gamma$-graded left $B^{\#}$-module $L^{\#}$ is finitely generated, and let $M$ be a left $C D G$-module over $B$. Then the natural morphism $\operatorname{Ext}_{C}^{I I}\left(L_{C}, M_{C}\right) \rightarrow \operatorname{Ext}_{C}\left(L_{C}, M_{C}\right)$ is an isomorphism provided that either

(i) the object $M$ belongs to the minimal triangulated subcategory of $\mathrm{D}^{\mathrm{co}}\left(B-\bmod ^{\mathrm{cdg}}\right)$ containing the objects $\operatorname{Hom}_{k}\left(F, k^{\vee}\right)$ for all $F \in H^{0}\left(\bmod _{\mathrm{fgp}}^{\mathrm{cdg}}-B\right)$ and closed under infinite products; or

(ii) the object L belongs to the minimal thick subcategory of $\mathrm{D}^{\mathrm{abs}}\left(B-\bmod _{\mathrm{fg}}^{\mathrm{cdg}}\right)$ containing the image of $H^{0}\left(B-\bmod _{\mathrm{fgp}}^{\mathrm{cdg}}\right)$. 
Proof. Part (i): The equivalence of categories

$$
H^{0}\left(C-\bmod _{\mathrm{inj}}^{\mathrm{dg}}\right)_{\mathrm{inj}} \simeq \mathrm{D}\left(C-\bmod ^{\mathrm{dg}}\right)
$$

makes the embedding functor $H^{0}\left(C-\bmod _{\text {inj }}^{\mathrm{dg}}\right)_{\text {inj }} \rightarrow \mathrm{D}^{\mathrm{co}}\left(C-\bmod ^{\mathrm{dg}}\right)$ right adjoint to the localization functor $\mathrm{D}^{\mathrm{co}}\left(C-\mathrm{mod}^{\mathrm{dg}}\right) \rightarrow \mathrm{D}\left(C-\right.$ mod $\left.^{\mathrm{dg}}\right)$. It follows that the functor $H^{0}\left(C-\bmod _{\text {inj }}^{\mathrm{dg}}\right)_{\text {inj }} \rightarrow \mathrm{D}^{\mathrm{co}}\left(C\right.$-mod $\left.{ }^{\mathrm{dg}}\right)$ preserves infinite products (also, all infinite products exist in the coderived category since it is compactly generated [Positselski $2011 \mathrm{~b}$, Theorem 3.11.2]). Since the category $H^{0}\left(C-\bmod _{\mathrm{inj}}^{\mathrm{dg}}\right)_{\mathrm{inj}}$ is the minimal triangulated subcategory of $H^{0}\left(C\right.$-mod $\left.{ }^{\mathrm{dg}}\right)$ containing the objects $\operatorname{Hom}_{k}\left(F_{C}, k^{\vee}\right)$ and closed under infinite products [loc. cit., Theorem 1.5], the assertion follows from Proposition A(i).

Part (ii): The equivalence of absolute derived categories

$$
\mathrm{D}^{\mathrm{abs}}\left(B-\bmod _{\mathrm{fg}}^{\mathrm{cdg}}\right) \simeq \mathrm{D}^{\mathrm{abs}}\left(C-\bmod _{\mathrm{fg}}^{\mathrm{dg}}\right)
$$

takes objects of the full subcategory $H^{0}\left(B-\bmod _{\mathrm{fgp}}^{\mathrm{cdg}}\right) \subset \mathrm{D}^{\mathrm{abs}}\left(B-\bmod _{\mathrm{fg}}^{\mathrm{cdg}}\right)$ to representable (and, consequently, perfect and h-projective) DG-modules in

$$
H^{0}\left(C-\bmod _{\mathrm{fgp}}^{\mathrm{dg}}\right) \subset \mathrm{D}^{\mathrm{abs}}\left(C-\bmod _{\mathrm{fg}}^{\mathrm{dg}}\right),
$$

so it remains to apply Proposition A(ii).

Proposition $\mathbf{B}^{\prime}$. Assume that the $\Gamma$-graded category $B^{\#}$ is right coherent. Let $N$ be a right $C D G$-module over $B$ whose underlying $\Gamma$-graded right $B^{\#}$-module $N^{\#}$ is finitely presented, and let $M$ be a left $C D G$-module over $B$. Then the natural morphism $\operatorname{Tor}^{C}\left(N_{C}, M_{C}\right) \rightarrow \operatorname{Tor}^{C, I I}\left(N_{C}, M_{C}\right)$ is an isomorphism provided that either

(i) the object $M$ belongs to the minimal triangulated subcategory of

$$
H^{0}\left(B-\bmod _{\mathrm{prj}}^{\mathrm{cdg}}\right) \subset \mathrm{D}^{\mathrm{ctr}}\left(B-\bmod ^{\mathrm{cdg}}\right)
$$

containing the image of $H^{0}\left(B-\bmod _{\mathrm{fgp}}^{\mathrm{cdg}}\right)$ and closed under infinite direct sums; or

(ii) the object $N$ belongs to the minimal thick subcategory of $\mathrm{D}^{\mathrm{abs}}\left(\bmod _{\mathrm{fp}}^{\mathrm{cdg}}-B\right)$ containing the image of $H^{0}\left(\bmod _{\mathrm{fgp}}^{\mathrm{cdg}}-B\right)$.

Proof. Similar to that of Proposition $\mathrm{A}^{\prime}$ and based on Proposition B.

Now assume that the commutative ring $k$ has finite weak homological dimension and all the $\Gamma$-graded $k$-modules of morphisms in the category $B^{\#}$ are flat. Clearly, the DG-categories of left and right CDG-modules over the CDG-category $B \otimes_{k} B^{\text {op }}$ are naturally equivalent, as are the DG-categories of left and right DG-modules over the DG-category $C \otimes_{k} C^{\text {op }}$. The DG-category of CDG-modules over $B \otimes_{k} B^{\text {op }}$ is also naturally equivalent to the DG-category of DG-modules over $C \otimes_{k} C^{\text {op }}$ 
[Polishchuk and Positselski 2012, Section 2.6]. As above, we denote by $M_{C}$ the DG-module over $C \otimes_{k} C^{\text {op }}$ corresponding to a CDG-module $M$ over $B \otimes_{k} B^{\text {op }}$.

To any left CDG-module $G$ and right CDG-module $F$ over $B$, one can assign the left CDG-module $G \otimes_{k} F$ and the right CDG-module $F \otimes_{k} G$ (corresponding to each other under the above equivalence) over the CDG-category $B \otimes_{k} B^{\text {op }}$. There are also the natural diagonal CDG-module $B$ over $B \otimes_{k} B^{\text {op }}$ and DG-module $C$ over $C \otimes_{k} C^{\text {op }}$ [loc. cit., Section 2.4]; these also correspond to each other with respect to the above equivalence of DG-categories.

For any DG-module $M_{C}$ over $C \otimes_{k} C^{\text {op }}$, we are interested in the comparison morphisms between the two kinds of Hochschild cohomology $H^{I I, *}\left(C, M_{C}\right) \rightarrow$ $H H^{*}\left(C, M_{C}\right)$ and Hochschild homology $H H_{*}\left(C, M_{C}\right) \rightarrow H H_{*}^{I I}\left(C, M_{C}\right)$ [loc. cit., formula (23)].

Proposition C. Assume that the $\Gamma$-graded category $B^{\#} \otimes_{k} B^{\# \text { op }}$ is Noetherian and the diagonal $\Gamma$-graded module $B^{\#}$ over it is finitely generated. Let $M$ be a $C D G$-module over $B \otimes_{k} B^{\text {op }}$. Then the natural morphism $H^{I I, *}\left(C, M_{C}\right) \rightarrow$ $H H^{*}\left(C, M_{C}\right)$ is an isomorphism provided that either

(i) the object $M$ belongs to the minimal triangulated subcategory of

$$
\mathrm{D}^{\mathrm{co}}\left(B \otimes_{k} B^{\mathrm{op}}-\bmod ^{\mathrm{cdg}}\right)
$$

containing the CDG-modules $\operatorname{Hom}_{k}\left(F \otimes_{k} G, k^{\vee}\right)$ for all $F \in H^{0}\left(\bmod _{\mathrm{fgp}}^{\mathrm{cdg}}-B\right)$ and $G \in H^{0}\left(B-\bmod _{\mathrm{fgp}}^{\mathrm{cdg}}\right)$ and closed under infinite products; or

(ii) the diagonal $C D G$-module $B$ over $B \otimes_{k} B^{\text {op }}$ belongs to the minimal thick subcategory of

$$
\mathrm{D}^{\mathrm{abs}}\left(B \otimes_{k} B^{\mathrm{op}}-\bmod _{\mathrm{fg}}^{\mathrm{cdg}}\right)
$$

containing the CDG-modules $G \otimes_{k} F$ for all $F \in H^{0}\left(\bmod _{\mathrm{fgp}}^{\mathrm{cdg}}-B\right)$ and $G \in$ $H^{0}\left(B-\bmod _{\mathrm{fgp}}^{\mathrm{cdg}}\right)$.

Proposition D. Assume that the $\Gamma$-graded category $B^{\#} \otimes_{k} B^{\# \text { op }}$ is coherent and the diagonal $\Gamma$-graded module $B^{\#}$ over it is finitely presented. Let $M$ be a $C D G$-module

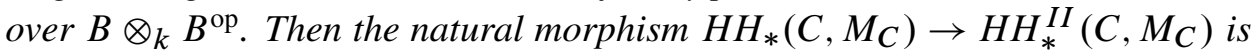
an isomorphism provided that either

(i) the object $M$ belongs to the minimal triangulated subcategory of

$$
H^{0}\left(B-\bmod _{\mathrm{prj}}^{\mathrm{cdg}}\right) \subset \mathrm{D}^{\mathrm{ctr}}\left(B \otimes_{k} B^{\mathrm{op}}-\bmod ^{\mathrm{cdg}}\right)
$$

containing the $C D G$-modules $G \otimes_{k} F$ for all $F \in H^{0}\left(\bmod _{\mathrm{fgp}}^{\mathrm{cdg}}-B\right)$ and $G \in$ $H^{0}\left(B-\bmod _{\mathrm{fgp}}^{\mathrm{cdg}}\right)$ and closed under infinite direct sums; or 
(ii) the diagonal $C D G$-module $B$ over $B \otimes_{k} B^{\text {op }}$ belongs to the minimal thick subcategory of $\mathrm{D}^{\mathrm{abs}}\left(B \otimes_{k} B^{\mathrm{op}}-\bmod _{\mathrm{fg}}^{\mathrm{cdg}}\right)$ containing the $C D G$-modules $G \otimes_{k} F$ for all $F \in H^{0}\left(\bmod _{\mathrm{fgp}}^{\mathrm{cdg}}-B\right)$ and $G \in H^{0}\left(B-\bmod _{\mathrm{fgp}}^{\mathrm{cdg}}\right)$.

Proofs of Propositions $C$ and D. Similar to the proofs of Propositions $\mathrm{A}^{\prime}$ and $\mathrm{B}^{\prime}$.

In particular, assume that the $\Gamma$-graded category $B^{\#} \otimes_{k} B^{\# \text { op }}$ is Noetherian and the diagonal $\Gamma$-graded module $B^{\#}$ over it is finitely generated. Suppose that the diagonal CDG-module $B$ over $B \otimes_{k} B^{\text {op }}$ belongs to the minimal thick subcategory of $\mathrm{D}^{\mathrm{abs}}\left(B \otimes_{k} B^{\mathrm{op}}-\bmod _{\mathrm{fg}}^{\mathrm{cdg}}\right)$ containing the CDG-modules $G \otimes_{k} F$ for all $F \in$ $H^{0}\left(\bmod _{\mathrm{fgp}}^{\mathrm{cdg}}-B\right)$ and $G \stackrel{\mathrm{f}}{\in} H^{0}\left(B-\bmod _{\mathrm{fgp}}^{\mathrm{cdg}}\right)$. Then, according to [Polishchuk and Positselski 2012, formulas (44-45) in Section 2.6] and parts (ii) of Propositions C and $\mathrm{D}$, there are natural isomorphisms

$$
\begin{gathered}
H H^{*}\left(C, M_{C}\right) \simeq H H^{I I, *}\left(C, M_{C}\right) \simeq H H^{I I, *}(B, M), \\
H H_{*}\left(C, M_{C}\right) \simeq H H_{*}^{I I}\left(C, M_{C}\right) \simeq H H_{*}^{I I}(B, M)
\end{gathered}
$$

for any CDG-module $M$ over $B \otimes_{k} B^{\text {op }}$. Specializing to the case of the diagonal CDG-module $M=B$ and DG-module $M_{C}=C$, we obtain

$$
\begin{aligned}
H H^{*}(C) & \simeq H H^{I I, *}(C) \simeq H H^{I I, *}(B), \\
H H_{*}(C) \simeq H H_{*}^{I I}(C) & \simeq H H_{*}^{I I}(B) .
\end{aligned}
$$

B.1.5. Locally free matrix factorizations. Let $k$ be a regular commutative Noetherian ring of finite Krull dimension and $X$ be an affine scheme of finite type over Spec $k$. Let $w \in \mathcal{O}(X)$ be a global regular function on $X$. Consider the $\mathbb{Z} / 2$-graded CDG-algebra $B$ over $k$ with $B^{0}=\mathcal{O}(X), B^{1}=0, d=0$, and $h=-w \in B^{0}$. We will find it convenient to denote the CDG-algebra $B$ simply by $(X, h)=(X,-w)$ (cf. Section 2.2 of the main body of this paper).

Then $C=\bmod _{\mathrm{fgp}}^{\mathrm{cdg}}-B$ is the $\mathbb{Z} / 2$-graded DG-category of locally free matrix factorizations of finite rank of the potential $w$ on $X$. Furthermore, one has $B \otimes_{k} B^{\text {op }}=$ $\left(X \times_{k} X, w_{2}-w_{1}\right)$, where $w_{i}=p_{i}^{*} w \in \mathcal{O}\left(X \times_{k} X\right), i=1,2$, and $p_{i}: X \times_{k} X \rightarrow X$ denote the coordinate projections. Let $\Delta: X \rightarrow X \times_{k} X$ be the diagonal embedding and $\Delta_{*} \mathcal{O}_{X}$ be the corresponding coherent sheaf on $X \times_{k} X$.

Consider the coherent matrix factorization of the potential $w_{2}-w_{1}$ on $X \times X$ whose even-degree component is the sheaf $\Delta_{*} \mathcal{O}_{X}$, while the odd-degree component vanishes. We will denote this "diagonal" matrix factorization simply by $\Delta_{*} \mathcal{O}_{X} \in H^{0}\left(\left(X \times_{k} X, w_{2}-w_{1}\right)-\bmod _{\mathrm{fg}}^{\mathrm{cdg}}\right)$. Applying the machinery of the previous sections leads to the following result (cf. [Polishchuk and Positselski 2012, Sections 4.8-4.10]). 
Corollary B.1.5. Suppose that the diagonal matrix factorization $\Delta_{*} \mathcal{O}_{X}$ belongs to the minimal thick subcategory of $\mathrm{D}^{\mathrm{abs}}\left(\left(X \times_{k} X, w_{2}-w_{1}\right)-\bmod _{\mathrm{fg}}^{\mathrm{cdg}}\right)$ containing the external tensor products of locally free matrix factorizations of finite rank $p_{1}^{*} G \otimes_{k} p_{2}^{*} F$ for all $G \in H^{0}\left((X,-w)\right.$ - $\left.\bmod _{\mathrm{fgp}}^{\mathrm{cdg}}\right)$ and $F \in H^{0}\left((X, w)-\bmod _{\mathrm{fgp}}^{\mathrm{cdg}}\right)$. Then the natural isomorphisms (8) hold for the CDG-algebra $B=(X, w)$ and the $D G$-category of locally free matrix factorizations $C=\bmod _{\mathrm{fgp}}^{\mathrm{cdg}}-B$.

Notice that the condition under which the conclusion of Corollary B.1.5 has been proven is a rather strong one, particularly when $X$ is not assumed to be a regular scheme. Then it is not even clear when or why the diagonal matrix factorization $\Delta_{*} \mathcal{O}_{X}$ should belong to the thick envelope of the full triangulated subcategory of locally free matrix factorizations

$$
H^{0}\left(\left(X \times_{k} X, w_{2}-w_{1}\right)-\bmod _{\mathrm{fgp}}^{\mathrm{cdg}}\right) \subset \mathrm{D}^{\mathrm{abs}}\left(\left(X \times_{k} X, w_{2}-w_{1}\right)-\bmod _{\mathrm{fg}}^{\mathrm{cdg}}\right)
$$

on $X \times_{k} X$, let alone to the thick subcategory generated by external tensor products of locally free matrix factorizations from the two copies of $X$.

B.1.6. Smooth stratifications. A scheme $X$ of finite type over a field $k$ is said to admit a smooth stratification [Efimov 2013] if it can be presented as a disjoint union of its locally closed subsets $X=\bigsqcup_{\alpha} S_{\alpha}$ so that each $S_{\alpha}$, when endowed with the structure of a reduced locally closed subscheme in $X$, becomes a smooth scheme over $k$. In particular, every scheme of finite type over a perfect field $k$ admits a smooth stratification, as any regular scheme of finite type over a perfect field is smooth over it [Grothendieck 1967, Corollaires 17.15.2 and 17.15.13]. Notice that a scheme of finite type over a field admits a smooth stratification if and only if its maximal reduced closed subscheme does.

The definition of a regular stratification of a Noetherian scheme is similar, except that the strata $S_{\alpha}$ are only required to be regular schemes in their reduced locally closed subscheme structures. Any scheme of finite type over a field admits a regular stratification [Grothendieck 1965, Scholie 7.8.3(iii)-(iv) and Proposition 7.8.6(i)].

Let $X$ be a smooth affine scheme over a field $k$ and $w \in \mathcal{O}(X)$ be a regular function on $X$. Set $X_{0}=\{w=0\} \subset X$ to be the zero locus of $w$. The following result is a slight generalization of [Polishchuk and Positselski 2012, Corollary 4.8.A] based on the above definitions.

Corollary B.1.6. Assume that there exists a closed subscheme $Z \subset X$ such that $w: X \backslash Z \rightarrow \mathbb{A}_{k}^{1}$ is a smooth morphism, $\left.w\right|_{Z}=0$, and the scheme $Z$ admits a smooth stratification over $k$. Then the conditions of Corollary B.1.5 are satisfied, so its conclusions apply.

Proof. According to the argument in [Polishchuk and Positselski 2012, Section 4.8], it suffices to show that the bounded derived category of coherent sheaves on $Z \times Z$ 
is generated by external tensor products of coherent sheaves on the two Cartesian factors. This is a particular case of the following lemma.

Lemma B.1.6. Let $Z^{\prime}$ and $Z^{\prime \prime}$ be schemes of finite type over a field $k$. Assume that the scheme $Z^{\prime}$ admits a smooth stratification. Then the bounded derived category of coherent sheaves $\mathrm{D}^{\mathrm{b}}\left(\left(Z^{\prime} \times Z^{\prime \prime}\right)\right.$-coh) on the Cartesian product $Z^{\prime} \times{ }_{k} Z^{\prime \prime}$ coincides with its minimal thick subcategory containing the external tensor products $\mathcal{K}^{\prime} \otimes_{k} \mathcal{K}^{\prime \prime}$ of coherent sheaves on $\mathcal{K}^{\prime}$ on $Z^{\prime}$ and $\mathcal{K}^{\prime \prime}$ on $Z^{\prime \prime}$.

Proof. One proceeds by induction on the total number of strata in a smooth stratification of $Z^{\prime}$ and a regular stratification of $Z^{\prime \prime}$. Clearly, one can replace $Z^{\prime}$ and $Z^{\prime \prime}$ with their maximal reduced closed subschemes. Now if $S_{\alpha_{0}}$ is an open stratum in $Z^{\prime}$ and $T_{\beta_{0}}$ is an open stratum in $Z^{\prime \prime}$, then $S_{\alpha_{0}}$ is smooth as an open subscheme in $Z^{\prime}$ and $T_{\beta_{0}}$ is regular as an open subscheme in $Z^{\prime \prime}$, while the induction assumption applies to $\left(Z^{\prime} \backslash S_{\alpha_{0}}\right) \times_{k} Z^{\prime \prime}$ and $Z^{\prime} \times_{k}\left(Z^{\prime \prime} \backslash T_{\beta_{0}}\right)$. The scheme $S_{\alpha_{0}} \times_{k} T_{\beta_{0}}$ is regular since it is smooth over a regular scheme. The rest of the argument is based on [Orlov 2011, Proposition 2.7] and follows the lines of [Lin and Pomerleano 2013, proof of Theorem 3.7].

B.2. Coherent matrix factorizations. In this section, we return to the notation system typical for the main body of this paper. The notion of a critical value of a regular function on a singular variety is defined in Section B.2.1. In Section B.2.2 we show that the external tensor product of coherent matrix factorizations is a fully faithful functor between the absolute derived categories and provide a sufficient condition for the pretriangulated extension of its DG-category version to be a quasiequivalence. The Hochschild cohomology of the DG-category corresponding to the absolute derived category of coherent matrix factorizations of a potential having no critical values but zero is computed in Section B.2.4.

The notion of cotensor product of complexes of quasicoherent sheaves and quasicoherent matrix factorizations is discussed in Sections B.2.5-B.2.6 and used in order to compute the Hochschild homology of the (same) DG-category of coherent matrix factorizations in Section B.2.7. The direct sum formula for the Hochschild (co)homology of the DG-categories of coherent matrix factorizations of a potential with several critical values is established in Section B.2.8.

In some sense, the results of this section (as compared to those of Section B.1) suggest that the DG-category corresponding to the absolute derived category of coherent matrix factorizations on a singular variety may be better behaved than the similar category of locally free matrix factorizations of finite rank. Other (and in some way related) arguments in support of the same conclusion are provided by the results of the papers [Lunts 2010; Efimov 2013] showing that the DG-category corresponding to the absolute derived category of coherent matrix factorizations is 
smooth (and even homotopically finitely presented), under suitable conditions on the field $k$. (Cf. the counterexample in Section 3.3.)

B.2.1. Noncritical functions. Let $k$ be a field and $X$ be a scheme of finite type over Spec $k$. Let $f \in \mathcal{O}(X)$ be a global regular function on $X$.

Let $Y$ be a scheme of finite type over Spec $k$ and $g \in \mathcal{O}(Y)$ be a global regular function. Let $p_{1}: X \times_{k} Y \rightarrow X$ and $p_{2}: X \times_{k} Y \rightarrow Y$ be the natural projections. Consider the regular function $f_{1}+g_{2}=p_{1}^{*} f+p_{2}^{*} g$ on $X \times_{k} Y$.

Suppose that $f: X \rightarrow \mathbb{A}_{k}^{1}$ is a flat morphism from $X$ to the affine line (when $k$ is algebraically closed, this means that the function $f-c$ is a local nonzero-divisor on $X$ for every $c \in k$ ). Then the morphism $f_{1}+g_{2}: X \times_{k} Y \rightarrow \mathbb{A}_{k}^{1}$ is also flat as it is the composition of two flat morphisms

$$
X \times_{k} Y \longrightarrow \mathbb{A}_{k}^{1} \times_{k} Y \longrightarrow \mathbb{A}_{k}^{1}
$$

(the former morphism being flat since the morphism $f: X \rightarrow \mathbb{A}_{k}^{1}$ is and the latter one because the polynomial $x+g$ does not divide zero in $B[x]$ for any commutative ring $B$ and element $g \in B$ ). In particular, it follows that the function $f_{1}+g_{2}$ is a local nonzero-divisor on the Cartesian product $X \times_{k} Y$.

A function $f \in \mathcal{O}(X)$ is said to be noncritical (or to have no critical values) if for any regular function $g \in \mathcal{O}(Y)$ on a scheme $Y$ of finite type over Spec $k$ the absolute derived category of coherent matrix factorizations $\mathrm{D}^{\mathrm{abs}}\left(\left(X \times_{k} Y, \mathcal{O}, f_{1}+g_{2}\right)\right.$-coh $)$ vanishes (i.e., is equivalent to the zero category). According to Remark 1.3 and Theorem 1.10(b), this condition is local in both $X$ and $Y$.

Therefore, given a scheme $X$ of finite type over Spec $k$ and a regular function $f \in \mathcal{O}(X)$, there is a unique maximal open subscheme $X_{f}^{\prime} \subset X$ where the function $f$ is noncritical. We will see below that the open subscheme $X_{f}^{\prime}$ is always dense in $X$ if the morphism $f: X \rightarrow \mathbb{A}_{k}^{1}$ is flat and the field $k$ has zero characteristic.

Similarly, there is a unique maximal open subscheme $\mathbb{A}_{k, f}^{1} \subset \mathbb{A}_{k}^{1}$ such that the restriction of $f$ to its full preimage in $X$ is noncritical. The scheme $\mathbb{A}_{k, f}^{1}$ is always nonempty if the field $k$ has zero characteristic. The points in the complement $\mathbb{A}_{k}^{1} \backslash \mathbb{A}_{k, f}^{1}$ are called the critical values of $f$. In particular, one says that $f$ has no critical values but zero if the restriction of $f$ to $f^{-1}\left(\mathbb{A}_{k}^{1} \backslash\{0\}\right) \subset X$ is noncritical.

Notice that when the schemes $X$ and $Y$ are separated and the morphism of schemes $f: X \rightarrow \mathbb{A}_{k}^{1}$ is flat, the category $\mathrm{D}^{\text {abs }}\left(\left(X \times_{k} Y, \mathcal{O}, f_{1}+g_{2}\right)\right.$-coh $)$ is equivalent to the triangulated category $\mathrm{D}_{\text {Sing }}^{\mathrm{b}}\left(\left\{f_{1}+g_{2}=0\right\} / X \times_{k} Y\right)$ of relative singularities of the zero locus of the function $f_{1}+g_{2}$ on $X \times_{k} Y$ (see Theorem 2.7).

Remark B.2.1. It would be interesting to have a geometric characterization of noncriticality of functions on singular schemes. For example, how does our definition of noncriticality relate to the condition that the differential of $f$ at every closed point $x \in X$ be a nonzero element of the Zariski cotangent space $T_{x}^{*} X$ ? We do not know this; cf. the smooth stratification approach below. 
Lemma B.2.1. Let $X=\bigsqcup_{\alpha} S_{\alpha}$ be a scheme of finite type over Spec $k$ presented as a disjoint union of its locally closed subsets, endowed with their reduced locally closed subscheme structures. Let $\mathcal{L}$ be a line bundle on $X$ and $w \in$ $\mathcal{L}(X)$ be its global section. In this setting, if the absolute derived categories $\mathrm{D}^{\text {abs }}\left(\left(S_{\alpha},\left.\mathcal{L}\right|_{S_{\alpha}},\left.w\right|_{S_{\alpha}}\right)\right.$-coh) vanish for all $\alpha$, then so does the absolute derived category $\mathrm{D}^{\mathrm{abs}}((X, \mathcal{L}, w)$-coh).

Proof. Proceeding by induction on the number of strata in the stratification $S_{\alpha}$, it suffices to consider the case when there are only two of them, namely, a closed subset $S \subset X$ and its open complement $X \backslash S$. One can also replace $X$ with its maximal reduced closed subscheme. Then the desired assertion follows from Theorem 1.10(b) since the triangulated category $\mathrm{D}^{\text {abs }}\left((X, \mathcal{L}, w)\right.$-coh $\left.{ }_{S}\right)$ is generated by the image of the natural functor $\mathrm{D}^{\text {abs }}\left(\left(S,\left.\mathcal{L}\right|_{S},\left.w\right|_{S}\right)\right.$-coh $) \rightarrow \mathrm{D}^{\text {abs }}((X, \mathcal{L}, w)$-coh $S)$.

Proposition B.2.1. Let $X$ be a scheme of finite type over Spec $k$ and $f \in \mathcal{O}(X)$ be a regular function on $X$. Let $X=\bigsqcup_{\alpha} S_{\alpha}$ be a smooth stratification of the scheme $X$ over $k$ (see Section B.1.6) such that the morphisms of schemes $\left.f\right|_{S_{\alpha}}: S_{\alpha} \rightarrow \mathbb{A}_{k}^{1}$ are smooth for all $\alpha$. Then the function $f$ is noncritical on $X$.

Proof. Let $Y$ be a scheme of finite type over Spec $k$ and $g \in \mathcal{O}(Y)$ be a regular function. We have to show that the triangulated category $\mathrm{D}^{\text {abs }}\left(\left(X \times_{k} Y, \mathcal{O}, f_{1}+g_{2}\right)\right.$-coh $)$ vanishes. Choosing a stratification of $Y$ by regular locally closed subschemes and applying Lemma B.2.1, one can assume that $X$ is smooth over $k$ and $Y$ is regular.

Then the scheme $X \times_{k} Y$ is also regular, the derivative of the function $f_{1}+g_{2} \in$ $\mathcal{O}\left(X \times{ }_{k} Y\right)$, viewed as an element of the Zariski cotangent space, does not vanish at any points where the function itself does (and, in a sense, at any other closed points, too), and it follows that the zero locus of $f_{1}+g_{2}$ in $X \times_{k} Y$ is also a regular scheme. It remains to use Theorem 2.7 (or [Orlov 2012, Theorem 3.5] and Corollary 2.4(c)).

It follows from Proposition B.2.1 that for any scheme of finite type $X$ with a smooth stratification $X=\bigsqcup_{\alpha} S_{\alpha}$ over Spec $k$ and any regular function $f \in \mathcal{O}(X)$, the set of critical values of the function $f$ on $X$ is contained in the union of the sets of critical values of the functions $f \mid S_{\alpha}$. In particular, if the characteristic of $k$ is zero, then all of these sets are finite.

B.2.2. External tensor products. Let $X^{\prime}$ and $X^{\prime \prime}$ be separated schemes of finite type over a field $k$, and let $w^{\prime} \in \mathcal{O}\left(X^{\prime}\right)$ and $w^{\prime \prime} \in \mathcal{O}\left(X^{\prime \prime}\right)$ be regular functions. Let $X^{\prime} \times_{k} X^{\prime \prime}$ be the Cartesian product, $p_{1}$ and $p_{2}$ be its natural projections onto the factors $X^{\prime}$ and $X^{\prime \prime}$, and $w_{1}^{\prime}+w_{2}^{\prime \prime}=p_{1}^{*} w^{\prime}+p_{2}^{*} w^{\prime \prime}$ be the related regular function 
on $X^{\prime} \times_{k} X^{\prime \prime}$. Then there is the external tensor product functor

$$
\begin{aligned}
\otimes_{k}: \mathrm{D}^{\mathrm{co}}\left(\left(X^{\prime}, \mathcal{O}, w^{\prime}\right)-\mathrm{qcoh}\right) \times \mathrm{D}^{\mathrm{co}}( & \left.\left(X^{\prime \prime}, \mathcal{O}, w^{\prime \prime}\right)-\mathrm{qcoh}\right) \\
& \longrightarrow \mathrm{D}^{\mathrm{co}}\left(\left(X^{\prime} \times_{k} X^{\prime \prime}, \mathcal{O}, w_{1}^{\prime}+w_{2}^{\prime \prime}\right)-\mathrm{qcoh}\right),
\end{aligned}
$$

which restricts to the similar functor

$$
\begin{aligned}
\otimes_{k}: \mathrm{D}^{\mathrm{abs}}\left(\left(X^{\prime}, \mathcal{O}, w^{\prime}\right)-\mathrm{coh}\right) \times \mathrm{D}^{\mathrm{abs}} & \left(\left(X^{\prime \prime}, \mathcal{O}, w^{\prime \prime}\right)-\mathrm{coh}\right) \\
& \longrightarrow \mathrm{D}^{\mathrm{abs}}\left(\left(X^{\prime} \times_{k} X^{\prime \prime}, \mathcal{O}, w_{1}^{\prime}+w_{2}^{\prime \prime}\right)-\mathrm{coh}\right)
\end{aligned}
$$

on coherent matrix factorizations.

Proposition B.2.2. Let $\mathcal{K}^{\prime}$ and $\mathcal{M}^{\prime}$ be coherent matrix factorizations of the potential $w^{\prime}$ on the scheme $X^{\prime}$, and let $\mathcal{K}^{\prime \prime}$ and $\mathcal{M}^{\prime \prime}$ be coherent matrix factorizations of the potential $w^{\prime \prime}$ on the scheme $X^{\prime \prime}$. Then the natural map of $\mathbb{Z} / 2$-graded $k$-vector spaces of morphisms

$$
\begin{aligned}
\operatorname{Hom}_{D^{\text {abs }}\left(\left(X^{\prime}, \mathcal{O}, w^{\prime}\right) \text {-coh }\right)}\left(\mathcal{K}^{\prime}, \mathcal{M}^{\prime}[*]\right) \otimes_{k} \operatorname{Hom}_{D^{a b s}\left(\left(X^{\prime \prime}, \mathcal{O}, w^{\prime \prime}\right)-\text { coh }\right)}\left(\mathcal{K}^{\prime \prime}, \mathcal{M}^{\prime \prime}[*]\right) \\
\longrightarrow \operatorname{Hom}_{\mathrm{D}^{\text {abs }}\left(\left(X^{\prime} \times_{k} X^{\prime \prime}, \mathcal{O}, w_{1}^{\prime}+w_{2}^{\prime \prime}\right)-\text { coh }\right)}\left(\mathcal{K}^{\prime} \otimes_{k} \mathcal{K}^{\prime \prime}, \mathcal{M}^{\prime} \otimes_{k} \mathcal{M}^{\prime \prime}[*]\right)
\end{aligned}
$$

induced by the additive functor of two arguments (10) is an isomorphism.

Proof. By Proposition 1.5(d), it suffices to show that the natural map

$$
\begin{array}{r}
\operatorname{Hom}_{\mathrm{D}^{\mathrm{co}}\left(\left(X^{\prime}, \mathcal{O}, w^{\prime}\right)-\mathrm{qcoh}\right)}\left(\mathcal{K}^{\prime}, \mathcal{M}^{\prime}[*]\right) \otimes_{k} \operatorname{Hom}_{\mathrm{D}^{\mathrm{co}}\left(\left(X^{\prime \prime}, \mathcal{O}, w^{\prime \prime}\right)-\mathrm{qcoh}\right)}\left(\mathcal{K}^{\prime \prime}, \mathcal{M}^{\prime \prime}[*]\right) \\
\longrightarrow \operatorname{Hom}_{\mathrm{D}^{\mathrm{co}}\left(\left(X^{\prime} \times_{k} X^{\prime \prime}, \mathcal{O}, w_{1}^{\prime}+w_{2}^{\prime \prime}\right)-\mathrm{qcoh}\right)}\left(\mathcal{K}^{\prime} \otimes_{k} \mathcal{K}^{\prime \prime}, \mathcal{M}^{\prime} \otimes_{k} \mathcal{M}^{\prime \prime}[*]\right)
\end{array}
$$

induced by the functor (9) is an isomorphism for any coherent matrix factorizations $\mathcal{K}^{\prime}, \mathcal{K}^{\prime \prime}$ and quasicoherent matrix factorizations $\mathcal{M}^{\prime}, \mathcal{M}^{\prime \prime}$ of the potentials $w^{\prime}$ and $w^{\prime \prime}$. One easily checks that the desired assertion holds for the Hom spaces in the homotopy categories of matrix factorizations (since it holds for morphisms between the external tensor products of coherent and quasicoherent sheaves).

Furthermore, one can assume the quasicoherent matrix factorizations $\mathcal{M}^{\prime}$ and $\mathcal{M}^{\prime \prime}$ to be injective. Then the Hom spaces in the left-hand side of the map (12) coincide with the similar Hom spaces computed in the homotopy categories of matrix factorizations. Let $\mathcal{I} \bullet$ be a right resolution of $\mathcal{M}^{\prime} \otimes_{k} \mathcal{M}^{\prime \prime}$ in the abelian category of quasicoherent matrix factorizations (and closed morphisms between them) consisting of injective matrix factorizations, and let $\mathcal{J}$ be the total matrix factorization of the complex $\mathcal{I}^{\bullet}$ constructed by taking infinite direct sums along the diagonals. Then the $k$-vector spaces of morphisms from $\mathcal{K}^{\prime} \otimes_{k} \mathcal{K}^{\prime \prime}$ into $\mathcal{J}$ in the homotopy category of matrix factorizations are isomorphic to the right-hand side of (12) [Positselski 2011b, Theorem 3.7]. 
It remains to show that the spaces of morphisms from $\mathcal{K}^{\prime} \otimes_{k} \mathcal{K}^{\prime \prime}$ to $\mathcal{M}^{\prime} \otimes_{k} \mathcal{M}^{\prime \prime}$ in the homotopy category of matrix factorizations are isomorphic to the similar spaces of morphisms from $\mathcal{K}^{\prime} \otimes_{k} \mathcal{K}^{\prime \prime}$ to $\mathcal{J}$. Indeed, taking the termwise Hom from $\mathcal{K}^{\prime} \otimes_{k} \mathcal{K}^{\prime \prime}$ preserves exactness of the sequence $0 \rightarrow \mathcal{M}^{\prime} \otimes_{k} \mathcal{M}^{\prime \prime} \rightarrow \mathcal{I}^{\bullet}$ since the higher Ext spaces from the components of $\mathcal{K}^{\prime} \otimes_{k} \mathcal{K}^{\prime \prime}$ into those of $\mathcal{M}^{\prime} \otimes_{k} \mathcal{M}^{\prime \prime}$ in the abelian category of quasicoherent sheaves on $X^{\prime} \otimes_{k} X^{\prime \prime}$ vanish. The latter assertion can be checked for affine schemes $X^{\prime}, X^{\prime \prime}$ using projective resolutions and then globally for the cohomology of quasicoherent sheaves using, e.g., the Čech approach.

Theorem B.2.2. Assume that the morphisms of schemes

$$
w^{\prime}: X^{\prime} \rightarrow \mathbb{A}_{k}^{1} \quad \text { and } \quad w^{\prime \prime}: X^{\prime \prime} \rightarrow \mathbb{A}_{k}^{1}
$$

are flat. Suppose that there exist closed subschemes $Z^{\prime} \subset X^{\prime}$ and $Z^{\prime \prime} \subset X^{\prime \prime}$ such that $\left.w^{\prime}\right|_{Z^{\prime}}=0=w_{Z^{\prime \prime}}^{\prime \prime}$, the functions $w^{\prime}$ and $w^{\prime \prime}$ are noncritical on $X^{\prime} \backslash Z^{\prime}$ and $X^{\prime \prime} \backslash Z^{\prime \prime}$, and the scheme $Z^{\prime}$ admits a smooth stratification over $k$. Then the absolute derived category $\mathrm{D}^{\mathrm{abs}}\left(\left(X^{\prime} \times_{k} X^{\prime \prime}, \mathcal{O}, w_{1}^{\prime}+w_{2}^{\prime \prime}\right)\right.$-coh) coincides with its minimal thick subcategory containing the image of the functor (10).

Proof. By the definition of noncriticality, one has

$$
\begin{aligned}
\mathrm{D}^{\mathrm{abs}}\left(\left(\left(\left(X^{\prime} \backslash Z^{\prime}\right) \times_{k} X^{\prime \prime}\right), \mathcal{O}, w_{1}^{\prime}\right.\right. & \left.\left.+w_{2}^{\prime \prime}\right)-\mathrm{coh}\right)=0 \\
& =\mathrm{D}^{\mathrm{abs}}\left(\left(\left(X^{\prime} \times_{k}\left(X^{\prime \prime} \backslash Z^{\prime \prime}\right)\right), \mathcal{O}, w_{1}^{\prime}+w_{2}^{\prime \prime}\right)-\mathrm{coh}\right) .
\end{aligned}
$$

Therefore, any coherent matrix factorization of the potential $w_{1}^{\prime}+w_{2}^{\prime \prime}$ on $X^{\prime} \times_{k} X^{\prime \prime}$ has its category-theoretic support inside $Z^{\prime} \times{ }_{k} Z^{\prime \prime}$, and is consequently isomorphic in $\mathrm{D}^{\text {abs }}\left(\left(X^{\prime} \times_{k} X^{\prime \prime}, \mathcal{O}, w_{1}^{\prime}+w_{2}^{\prime \prime}\right)\right.$-coh $)$ to a direct summand of an object represented by a coherent matrix factorization supported set-theoretically inside $Z^{\prime} \times_{k} Z^{\prime \prime}$ (see Corollary 1.10(b)). It follows that the triangulated category

$$
\mathrm{D}^{\mathrm{abs}}\left(\left(X^{\prime} \times_{k} X^{\prime \prime}, O, w_{1}^{\prime}+w_{2}^{\prime \prime}\right)-\mathrm{coh}\right)
$$

is generated by the direct images of coherent matrix factorizations of the zero potential from the closed embedding $Z^{\prime} \times_{k} Z^{\prime \prime} \rightarrow X^{\prime} \times_{k} X^{\prime \prime}$.

Furthermore, let $X_{0}^{\prime}, X_{0}^{\prime \prime}$, and $Y_{0}$ denote the zero loci of the functions $w^{\prime}, w^{\prime \prime}$, and $w_{1}^{\prime}+w_{2}^{\prime \prime}$ on $X^{\prime}, X^{\prime \prime}$, and $X^{\prime} \times_{k} X^{\prime \prime}$, respectively. Denote the natural closed embeddings by $i^{\prime}: X_{0}^{\prime} \rightarrow X^{\prime}, i^{\prime \prime}: X_{0}^{\prime \prime} \rightarrow X^{\prime \prime}, \iota: X_{0}^{\prime} \times X_{0}^{\prime \prime} \rightarrow Y_{0}$, and $h: Y_{0} \rightarrow$ $X^{\prime} \times X^{\prime \prime}$. The external tensor product functor (cf. [Polishchuk and Positselski 2012, Lemma 4.8.B])

$$
\otimes_{k}: \mathrm{D}_{\text {Sing }}^{\mathrm{b}}\left(X_{0}^{\prime} / X^{\prime}\right) \times \mathrm{D}_{\text {Sing }}^{\mathrm{b}}\left(X_{0}^{\prime \prime} / X^{\prime \prime}\right) \longrightarrow \mathrm{D}_{\text {Sing }}^{\mathrm{b}}\left(Y_{0} /\left(X^{\prime} \times_{k} X^{\prime \prime}\right)\right)
$$

is well-defined since for any bounded complexes of coherent sheaves $\mathcal{F}^{\bullet}$ on $X^{\prime}$ and $\mathcal{K}^{\bullet}$ on $X_{0}^{\prime \prime}$ one has $\iota_{*}\left(\mathbb{L} i^{\prime *} \mathcal{F}^{\bullet} \otimes_{k} \mathcal{K}^{\bullet}\right) \simeq \mathbb{L} h^{*}\left(\left(\operatorname{id}_{X^{\prime}} \times i^{\prime \prime}\right)_{*}\left(\mathcal{F}^{\bullet} \otimes_{k} \mathcal{K}^{\bullet}\right)\right)$. Indeed, the 
square diagram of closed embeddings

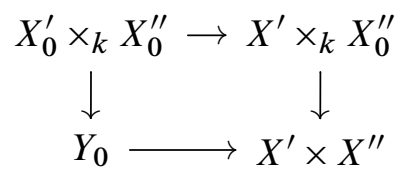

is Cartesian and the higher derived tensor products related to the construction of this relative Cartesian product of schemes all vanish.

The functor $\Upsilon: \mathrm{D}_{\text {Sing }}^{\mathrm{b}}\left(Y_{0} /\left(X^{\prime} \times_{k} X^{\prime \prime}\right)\right) \rightarrow \mathrm{D}^{\mathrm{abs}}\left(\left(X^{\prime} \times_{k} X^{\prime \prime}, \mathcal{O}, w_{1}^{\prime}+w_{2}^{\prime \prime}\right)\right.$-coh) (see Section 2.7) and the similar functors for the potentials $w^{\prime}$ and $w^{\prime \prime}$ on $X^{\prime}$ and $X^{\prime \prime}$ transform the external product functor (10) into the external tensor product functor (13). By the assumption, one has $Z^{\prime} \subset X_{0}^{\prime}$ and $Z^{\prime \prime} \subset X_{0}^{\prime \prime}$. It remains to apply Lemma B.1.6 in order to finish the proof of the theorem.

B.2.3. Internal Hom of matrix factorizations. Let $X$ be a separated Noetherian scheme. Let $\mathcal{L}$ be a line bundle on $X$ and $w^{\prime}, w^{\prime \prime} \in \mathcal{L}(X)$ be its global sections. Then given a matrix factorization $\mathcal{U}^{0} \rightarrow \mathcal{U}^{1} \otimes \mathcal{L}^{\otimes 1 / 2} \rightarrow \mathcal{U}^{0} \otimes \mathcal{O}_{X} \mathcal{L}$ of the potential $w^{\prime}$ and a matrix factorization $\mathcal{V}^{0} \rightarrow \mathcal{V}^{1} \otimes \mathcal{L}^{\otimes 1 / 2} \rightarrow \mathcal{V}^{0} \otimes_{\mathcal{O}_{X}} \mathcal{L}$ of the potential $w^{\prime \prime}$ on the scheme $X$ (in the symbolic notation of Section 2.2), one can construct the matrix factorization

$$
\begin{aligned}
\mathcal{U}^{0} \otimes_{\mathcal{O}_{X}} \mathcal{V}^{0} \oplus \mathcal{U}^{1} \otimes_{\mathcal{O}_{X}} \mathcal{V}^{1} & \\
\longrightarrow & \mathcal{U}^{1} \otimes \mathcal{L}^{\otimes 1 / 2} \otimes_{\mathcal{O}_{X}} \mathcal{V}^{0} \oplus \mathcal{U}^{0} \otimes_{\mathcal{O}_{X}} \mathcal{V}^{1} \otimes \mathcal{L}^{\otimes 1 / 2} \\
& \longrightarrow \mathcal{U}^{0} \otimes_{\mathcal{O}_{X}} \mathcal{V}^{0} \otimes_{\mathcal{O}_{X}} \mathcal{L} \oplus \mathcal{U}^{1} \otimes_{\mathcal{O}_{X}} \mathcal{V}^{1} \otimes_{\mathcal{O}_{X}} \mathcal{L}
\end{aligned}
$$

of the potential $w^{\prime}+w^{\prime \prime}$ on $X$. Here the tensor product $\mathcal{U}^{1} \otimes_{\mathcal{O}_{X}} \mathcal{V}^{1}$ is defined as the sheaf $\left(\mathcal{U}^{1} \otimes \mathcal{L}^{\otimes 1 / 2}\right) \otimes_{\mathcal{O}_{X}}\left(\mathcal{V}^{1} \otimes \mathcal{L}^{\otimes 1 / 2}\right) \otimes_{\mathcal{O}_{X}} \mathcal{L}^{\otimes-1}$ on $X$, while the differential on the tensor product of matrix factorizations is given by the conventional rule $d(u \otimes v)=d(u) \otimes v+(-1)^{|u|} u \otimes d(v)$.

We denote the matrix factorization so obtained by $\mathcal{U} \otimes_{\mathcal{O}_{X}} \mathcal{V}$ and call it the tensor product of two matrix factorizations $\mathcal{U}$ and $\mathcal{V}$ of two sections $w^{\prime}$ and $w^{\prime \prime}$ of the same line bundle $\mathcal{L}$ on a scheme $X$. Restricting to the cases when one or both matrix factorizations are flat, and passing to the coderived categories, one obtains the induced tensor product functors

$$
\begin{aligned}
\otimes_{\mathcal{O}_{X}}: \mathrm{D}^{\mathrm{co}}\left(\left(X, \mathcal{L}, w^{\prime}\right)-\mathrm{q} \operatorname{coh}_{\mathrm{fl}}\right) \times \mathrm{D}^{\mathrm{co}}\left(\left(X, \mathcal{L}, w^{\prime \prime}\right)-\mathrm{qcoh}_{\mathrm{fl}}\right) \\
\longrightarrow \mathrm{D}^{\mathrm{co}}\left(\left(X, \mathcal{L}, w^{\prime}+w^{\prime \prime}\right)-\mathrm{qcoh}_{\mathrm{fl}}\right)
\end{aligned}
$$

and

$\otimes_{\mathcal{O}_{X}}: \mathrm{D}^{\mathrm{co}}\left(\left(X, \mathcal{L}, w^{\prime}\right)-\mathrm{qcoh} \mathrm{fl}_{\mathrm{fl}}\right) \times \mathrm{D}^{\mathrm{co}}\left(\left(X, \mathcal{L}, w^{\prime \prime}\right)\right.$-qcoh $)$

$$
\longrightarrow \mathrm{D}^{\mathrm{co}}\left(\left(X, \mathcal{L}, w^{\prime}+w^{\prime \prime}\right) \text {-qcoh }\right) .
$$


The functors (14) and (15) are well-defined since the tensor product with a flat (quasicoherent) matrix factorization takes a short exact sequence of flat matrix factorizations to a short exact sequence of flat matrix factorizations, the tensor product with a flat matrix factorization takes a short exact sequence of quasicoherent matrix factorizations to a short exact sequence of quasicoherent matrix factorizations, and the tensor product with a quasicoherent matrix factorization takes a short exact sequence of flat matrix factorizations to a short exact sequence of quasicoherent matrix factorizations. Also, the tensor product functor preserves infinite direct sums.

Given a quasicoherent matrix factorization $\mathcal{U}^{0} \rightarrow \mathcal{U}^{1} \otimes \mathcal{L}^{\otimes 1 / 2} \rightarrow \mathcal{U}^{0} \otimes_{\mathcal{O}_{X}} \mathcal{L}$ of a potential $w^{\prime} \in \mathcal{L}(X)$ and a quasicoherent matrix factorization $\mathcal{V}^{0} \rightarrow \mathcal{V}^{1} \otimes \mathcal{L}^{\otimes 1 / 2} \rightarrow$ $\mathcal{V}^{1} \otimes_{\mathcal{O}_{X}} \mathcal{L}$ of a potential $w^{\prime \prime} \in \mathcal{L}(X)$ on the scheme $X$, one can construct the quasicoherent matrix factorization

$$
\begin{aligned}
\mathcal{H o m}_{X \text {-qc }}\left(\mathcal{U}^{0}, \mathcal{V}^{0}\right) \oplus \mathcal{H o m}_{X \text {-qc }}\left(\mathcal{U}^{1}, \mathcal{V}^{1}\right) \\
\longrightarrow \mathcal{H o m}_{X \text {-qc }}\left(\mathcal{U}^{0}, \mathcal{V}^{1} \otimes \mathcal{L}^{\otimes 1 / 2}\right) \oplus \mathcal{H o m}_{X \text {-qc }}\left(\mathcal{U}^{1}, \mathcal{V}^{0}\right) \otimes \mathcal{L}^{\otimes 1 / 2} \\
\longrightarrow \mathcal{H o m}_{X \text {-qc }}\left(\mathcal{U}^{0}, \mathcal{V}^{0}\right) \otimes_{\mathcal{O}_{X}} \mathcal{L} \oplus \mathcal{H} o m_{X \text {-qc }}\left(\mathcal{U}^{1}, \mathcal{V}^{1}\right) \otimes_{\mathcal{O}_{X}} \mathcal{L}
\end{aligned}
$$

of the potential $w^{\prime \prime}-w^{\prime}$ on $X$. Here the sheaf $\mathcal{H o m}_{X \text {-qc }}\left(\mathcal{U}^{1}, \mathcal{V}^{0}\right) \otimes \mathcal{L}^{\otimes 1 / 2}$ is defined as the tensor product $\mathcal{H}^{\circ} m_{X \text {-qc }}\left(\mathcal{U}^{1} \otimes \mathcal{L}^{\otimes 1 / 2}, \mathcal{V}^{0}\right) \otimes_{\mathcal{O}_{X}} \mathcal{L}$, while the differential on the internal Hom is given by the conventional rule $d(g)(u)=d(g(u))-(-1)^{|g|} g(d(u))$.

We denote the matrix factorization so obtained by $\mathcal{H o m}_{X \text {-qc }}(\mathcal{U}, \mathcal{V})$ and call it the matrix factorization of quasicoherent internal Hom between the quasicoherent matrix factorizations $\mathcal{U}$ and $\mathcal{V}$ of two sections $w^{\prime}$ and $w^{\prime \prime}$ of the same line bundle $\mathcal{L}$ on a scheme $X$. Restricting to the case when the matrix factorization in the second argument is injective, one obtains the induced internal Hom functor

$$
\begin{aligned}
\mathcal{H o m}_{X \text {-qc }}: \mathrm{D}^{\mathrm{abs}}\left(\left(X, \mathcal{L}, w^{\prime}\right)-\mathrm{qcoh}\right)^{\mathrm{op}} \times H^{0}\left(\left(X, \mathcal{L}, w^{\prime \prime}\right)-\text { qcoh }_{\text {inj }}\right) \\
\longrightarrow \mathrm{D}^{\mathrm{abs}}\left(\left(X, \mathcal{L}, w^{\prime \prime}-w^{\prime}\right) \text {-qcoh }\right),
\end{aligned}
$$

which can be also viewed as the right derived internal Hom functor

$$
\begin{aligned}
\mathbb{R} \text { Hom }_{X \text {-qc }}: D^{\text {abs }}\left(\left(X, \mathcal{L}, w^{\prime}\right) \text {-qcoh }\right)^{\mathrm{op}} \times \mathrm{D}^{\mathrm{co}}\left(\left(X, \mathcal{L}, w^{\prime \prime}\right) \text {-qcoh }\right) \\
\longrightarrow \mathrm{D}^{\mathrm{abs}}\left(\left(X, \mathcal{L}, w^{\prime \prime}-w^{\prime}\right) \text {-qcoh }\right) .
\end{aligned}
$$

Remark B.2.3. Alternatively, one could restrict the quasicoherent internal Hom functor to pairs of quasicoherent matrix factorizations which are both injective, obtaining the triangulated functor

$\mathcal{H o m}_{X-\mathrm{qc}}: H^{0}\left(\left(X, \mathcal{L}, w^{\prime}\right)-\mathrm{qcoh}{ }_{\text {inj }}\right)^{\mathrm{op}} \times H^{0}\left(\left(X, \mathcal{L}, w^{\prime \prime}\right)-\mathrm{qcoh}_{\text {inj }}\right)$

$$
\longrightarrow H^{0}\left(\left(X, \mathcal{L}, w^{\prime \prime}-w^{\prime}\right)-\mathrm{q} \operatorname{coh}_{\mathrm{fl}}\right),
$$


which can be also viewed as a derived internal Hom functor

$\mathbb{L} \mathbb{R} \mathcal{H o m}_{X \text {-qc }}: \mathrm{D}^{\mathrm{co}}\left(\left(X, \mathcal{L}, w^{\prime}\right)-\mathrm{qcoh}\right)^{\mathrm{op}} \times \mathrm{D}^{\mathrm{co}}\left(\left(X, \mathcal{L}, w^{\prime \prime}\right)-\mathrm{qcoh}\right)$

$$
\longrightarrow \mathrm{D}^{\mathrm{abs}}\left(\left(X, \mathcal{L}, w^{\prime \prime}-w^{\prime}\right)-\mathrm{qcoh}_{\mathrm{fl}}\right)
$$

that is a left derived functor in its first argument and a right derived functor in the second one. Notice that the derived functor so obtained does not agree with the right derived functor defined above; i.e., the composition of the functor (19) with the natural fully faithful functor $\mathrm{D}^{\mathrm{abs}}\left(\left(X, \mathcal{L}, w^{\prime \prime}-w^{\prime}\right)\right.$-qcoh $\left.\mathrm{qfl}_{\mathrm{fl}}\right) \rightarrow \mathrm{D}^{\mathrm{abs}}\left(\left(X, \mathcal{L}, w^{\prime \prime}-w^{\prime}\right)\right.$-qcoh $)$ and the Verdier localization functor $\mathrm{D}^{\mathrm{abs}}\left(\left(X, \mathcal{L}, w^{\prime}\right)\right.$-qcoh $) \rightarrow \mathrm{D}^{\mathrm{co}}\left(\left(X, \mathcal{L}, w^{\prime}\right)\right.$-qcoh $)$ is not isomorphic to the functor (17).

In particular, when $w^{\prime}=w^{\prime \prime}$, the functors (16) and (17) take values in the absolute derived category of quasicoherent matrix factorizations of the zero potential $0 \in \mathcal{L}(X)$. The objects of this category are simply complexes of quasicoherent sheaves $\mathcal{M}^{\bullet}$ on $X$ endowed with a 2-periodicity isomorphism $\mathcal{M}^{\bullet}[2] \simeq \mathcal{M}^{\bullet} \otimes_{\mathcal{O}_{X}} \mathcal{L}$. So there is a natural forgetful functor

$$
\mathrm{D}^{\mathrm{co}}((X, \mathcal{L}, 0) \text {-qcoh }) \longrightarrow \mathrm{D}^{\mathrm{co}}(X \text {-qcoh })
$$

and the similar functors acting on the homotopy, absolute derived, etc. categories of flat, coherent, locally free, etc. matrix factorizations.

Furthermore, there is the derived global sections functor

$$
\mathbb{R} \Gamma(X,-): \mathrm{D}^{\mathrm{co}}(X \text {-qcoh }) \longrightarrow \mathrm{D}(\mathbb{Z} \text {-mod })
$$

taking values in the derived category of abelian groups and defined using either the injective resolutions or the Čech construction (see Sections 1.8-1.9). In fact, the functor (21) factorizes through the conventional derived category $\mathrm{D}(X$-qcoh).

Composing the forgetful functor with the functor of underived global sections of complexes of quasicoherent sheaves, one obtains a triangulated functor

$$
\Gamma(X,-): H^{0}((X, \mathcal{L}, 0) \text {-qcoh }) \longrightarrow \mathrm{D}(\mathbb{Z} \text {-mod }) .
$$

Alternatively, the functor (22) can be defined as the functor $\operatorname{Hom}_{(X, \mathcal{L}, 0) \text {-qcoh }}\left(\mathcal{O}_{X},-\right)$, where the structure sheaf $\mathcal{O}_{X}$ is viewed as a matrix factorization $\left(\mathcal{U}^{0}, \mathcal{U}^{1}\right)$ of the potential $0 \in \mathcal{L}(X)$ with the components $\mathcal{U}^{0}=\mathcal{O}_{X}$ and $\mathcal{U}^{1} \otimes \mathcal{L}^{\otimes 1 / 2}=0$.

Similarly, composing the functors (20) and (21), one obtains a triangulated functor

$$
\mathbb{R} \Gamma(X,-): \mathrm{D}^{\mathrm{co}}((X, \mathcal{L}, 0) \text {-qcoh }) \longrightarrow \mathrm{D}(\mathbb{Z} \text {-mod }),
$$

which can be alternatively described as the functor $\operatorname{Hom}_{D^{c o}((X, \mathcal{L}, 0) \text {-qcoh })}\left(\mathcal{O}_{X},-[*]\right)$. In the case when $\mathcal{L}=\mathcal{O}_{X}$, the functors (22) and (23) can be viewed as taking values in the derived category of $\mathbb{Z} / 2$-graded (2-periodic) complexes of abelian groups. 
For any quasicoherent matrix factorizations $\mathcal{K}$ and $\mathcal{M}$ of a potential $w \in \mathcal{L}(X)$ on the scheme $X$ there is a natural isomorphism of complexes of abelian groups

$$
\operatorname{Hom}_{(X, \mathcal{L}, w)-\mathrm{qcoh}}(\mathcal{K}, \mathcal{M}) \simeq \Gamma\left(X, \mathcal{H o m}_{X \text {-qc }}(\mathcal{K}, \mathcal{M})\right),
$$

and more generally, for any quasicoherent matrix factorizations $\mathcal{K}$ and $\mathcal{E}$ of potentials $w^{\prime}$ and $w^{\prime \prime} \in \mathcal{L}(X)$ and a quasicoherent matrix factorization $\mathcal{M}$ of the potential $w^{\prime}+w^{\prime \prime}$ on the scheme $X$ there is a natural isomorphism of complexes

$$
\operatorname{Hom}_{\left(X, \mathcal{L}, w^{\prime}+w^{\prime \prime}\right)-\mathrm{qcoh}}\left(\mathcal{K} \otimes_{\mathcal{O}_{X}} \mathcal{E}, \mathcal{M}\right) \simeq \operatorname{Hom}_{\left(X, \mathcal{L}, w^{\prime \prime}\right)-\mathrm{qcoh}}\left(\mathcal{E}, \mathcal{H} \mathrm{H}_{X-\mathrm{qc}}(\mathcal{K}, \mathcal{M})\right) .
$$

Lemma B.2.3. Let $\mathcal{K}$ be a quasicoherent matrix factorization and $\mathcal{M}$ be an injective quasicoherent matrix factorization of a potential $w \in \mathcal{L}(X)$. Let

$$
\mathcal{H o m}_{X \text {-qc }}(\mathcal{K}, \mathcal{M}) \longrightarrow \mathcal{J}
$$

be a closed morphism with a coacyclic cone between quasicoherent matrix factorizations of the potential $0 \in \mathcal{L}(X)$ from the matrix factorization of quasicoherent internal Hom into an injective matrix factorization $\mathcal{J}$. Then the induced morphism

$$
\Gamma\left(X, \mathcal{H o m}_{X \text {-qc }}(\mathcal{K}, \mathcal{M})\right) \longrightarrow \Gamma(X, \mathcal{J})
$$

is a quasi-isomorphism of complexes of abelian groups.

Proof. Let $0 \rightarrow \mathcal{H o m}_{X \text {-qc }}(\mathcal{K}, \mathcal{M}) \rightarrow \mathcal{I}^{\bullet}$ be a right resolution of the matrix factorization $\mathcal{H o m}_{X \text {-qc }}(\mathcal{K}, \mathcal{M})$ by injective matrix factorization $\mathcal{I}^{i}$. Then one can take $\mathcal{J}$ to be the total matrix factorization of the complex $\mathcal{I}^{\bullet}$ constructed by passing to the infinite direct sums along the diagonals. Notice that the functor of global sections of quasicoherent sheaves on $X$ commutes with the infinite direct sums. It remains to show that the functor $\Gamma(X,-)=\operatorname{Hom}_{\left(X, \mathcal{L}, w^{\prime \prime}\right) \text {-qcoh }}\left(\mathcal{O}_{X},-\right)$ preserves the exactness of the sequence $0 \rightarrow \mathcal{H o m}_{X \text {-qc }}(\mathcal{K}, \mathcal{M}) \rightarrow \mathcal{I}^{\bullet}$ (cf. the proof of Proposition B.2.2).

In fact, we claim that the Ext groups from flat quasicoherent sheaves to the components of $\mathcal{H o m}_{X \text {-qc }}(\mathcal{K}, \mathcal{M})$ vanish in the abelian category $X$-qcoh. This assertion follows from the results of [Positselski 2012, Lemma 2.5.3(c) and Corollary 4.1.9(b)] (the argument is based essentially on the above Lemma A.1).

We recall the constructions of the (underived and derived) direct and inverse image functors for matrix factorizations from Sections 1.8-1.9 and 3.5-3.6. In addition to the conventional adjunction of the (underived) direct and inverse image functors $f_{*}$ and $f^{*}$ (as mentioned in Section 1.8), there is also the "internal Hom adjunction", formulated as follows.

Let $f: Z \rightarrow Y$ be a morphism of separated Noetherian schemes, $\mathcal{L}$ be a line bundle on $Y$, and $w^{\prime}, w^{\prime \prime} \in \mathcal{L}(Y)$ be two global sections. Let $\mathcal{K} \in H^{0}\left(\left(Y, \mathcal{L}, w^{\prime}\right)\right.$-qcoh $)$ 
and $\mathcal{M} \in H^{0}\left(\left(Z, f^{*} \mathcal{L}, f^{*} w^{\prime \prime}\right)\right.$-qcoh) be quasicoherent matrix factorizations on $Y$ and $Z$. Then there is a natural isomorphism

$$
f_{*} \mathcal{H o m}_{Z-\mathrm{qc}}\left(f^{*} \mathcal{K}, \mathcal{M}\right) \simeq \mathcal{H o m}_{Y-\mathrm{qc}}\left(\mathcal{K}, f_{*} \mathcal{M}\right)
$$

of quasicoherent matrix factorizations of the potential $w^{\prime \prime}-w^{\prime}$ on $Y$.

Now let $X$ be a separated scheme of finite type over a field $k$, and let $w^{\prime}, w^{\prime \prime} \in$ $\mathcal{O}(X)$ be two global regular functions on $X$. Denote by $p_{1}$ and $p_{2}$ the natural projections $X \times_{k} X \rightrightarrows X$, and consider the regular function $w_{1}^{\prime}+w_{2}^{\prime \prime}=p_{1}^{*} w^{\prime}+p_{2}^{*} w^{\prime \prime}$ on $X \times_{k} X$. Let $\Delta: X \rightarrow X \times_{k} X$ denote the diagonal map.

Let $\mathcal{N}$ and $\mathcal{K}$ be quasicoherent matrix factorizations of the potentials $w^{\prime}$ and $w^{\prime \prime}$ on $X$. Then there is a natural isomorphism $\mathcal{N} \otimes_{\mathcal{O}_{X}} \mathcal{K} \simeq \Delta^{*}\left(\mathcal{N} \otimes_{k} \mathcal{K}\right)$ of matrix factorizations of the potential $w^{\prime}+w^{\prime \prime}$ on $X$. Therefore, given a quasicoherent matrix factorization $\mathcal{M}$ of the potential $w^{\prime}+w^{\prime \prime} \in \mathcal{O}(X)$, one has a natural isomorphism of $\mathbb{Z} / 2$-graded complexes of abelian groups

$$
\begin{aligned}
& \operatorname{Hom}_{\left(X, \mathcal{O}, w^{\prime \prime}\right)-\mathrm{qcoh}}\left(\mathcal{K}, \mathcal{H o m}_{X-\mathrm{qc}}(\mathcal{N}, \mathcal{M})\right) \\
& \simeq \operatorname{Hom}_{\left(X \times_{k} X, \mathcal{O}, w_{1}^{\prime}+w_{2}^{\prime \prime}\right) \text {-qcoh }}\left(\mathcal{N} \otimes_{k} \mathcal{K}, \Delta_{*} \mathcal{M}\right) .
\end{aligned}
$$

Proposition B.2.3. (a) Assume that the matrix factorization $\mathcal{N}$ is coherent and the matrix factorization $\mathcal{M}$ is injective. Let $\Delta_{*} \mathcal{M} \rightarrow \mathcal{J}$ be a closed morphism with a coacyclic cone between quasicoherent matrix factorizations of the potential $w_{1}^{\prime}+w_{2}^{\prime \prime}$ on $X \times_{k} X$ from the direct image $\Delta_{*} \mathcal{M}$ into an injective matrix factorization $\mathcal{J}$. Then there is a natural closed morphism with a coacyclic cone $\mathcal{H}_{\text {om }}$-qc $(\mathcal{N}, \mathcal{M}) \rightarrow$ $p_{2 *} \mathcal{H o m}_{X \times_{k} X \text {-qc }}\left(p_{1}^{*} \mathcal{N}, \mathcal{J}\right)$ of quasicoherent matrix factorizations of the potential $w^{\prime \prime}$ on $X$, and the matrix factorization $p_{2 *} \operatorname{Hom}_{X \times_{k} X \text {-qc }}\left(p_{1}^{*} \mathcal{N}, \mathcal{J}\right)$ is injective.

(b) There is a natural isomorphism of $\mathbb{Z} / 2$-graded complexes of abelian groups

$$
\begin{aligned}
\operatorname{Hom}_{\left(X, \mathcal{O}, w^{\prime \prime}\right) \text {-qcoh }}\left(\mathcal{K}, p_{2 *} \mathcal{H o m}_{X \times_{k}} X \text {-qc }\left(p_{1}^{*} \mathcal{N}, \mathcal{J}\right)\right) \\
\simeq \operatorname{Hom}_{\left(X \times_{k} X, \mathcal{O}, w_{1}^{\prime}+w_{2}^{\prime \prime}\right) \text {-qcoh }}\left(\mathcal{N} \otimes_{k} \mathcal{K}, \mathcal{J}\right) .
\end{aligned}
$$

Proof. Part (a): The desired closed morphism is provided by the composition

$$
\begin{aligned}
\mathcal{H o m}_{X \text {-qc }}(\mathcal{N}, \mathcal{M}) \simeq p_{2 *} \Delta_{*} \mathcal{H o m}_{X \text {-qc }}\left(\Delta^{*} p_{1}^{*} \mathcal{N}, \mathcal{M}\right) \\
\quad \simeq p_{2 *} \mathcal{H o m}_{X \times_{k} X \text {-qc }}\left(p_{1}^{*} \mathcal{N}, \Delta_{*} \mathcal{M}\right) \longrightarrow p_{2 *} \mathcal{H o m}_{X \times_{k} X \text {-qc }}\left(p_{1}^{*} \mathcal{N}, \mathcal{J}\right) .
\end{aligned}
$$

To prove that this morphism has a coacyclic cone, pick a right resolution $\mathcal{I}^{\bullet}$ of the matrix factorization $\Delta_{*} \mathcal{M}$ on $X \times_{k} X$ by injective matrix factorizations, and take $\mathcal{J}$ to be the totalization of the complex of matrix factorizations $\mathcal{I} \cdot$ constructed by passing to the infinite direct sums along the diagonals.

Then the complex of matrix factorizations $0 \rightarrow \mathcal{H o m}_{X \times_{k} X \text {-qc }}\left(p_{1}^{*} \mathcal{N}, \Delta_{*} \mathcal{M}\right) \rightarrow$ $\mathcal{H o m}_{X \times_{k} X \text {-qc }}\left(p_{1}^{*} \mathcal{N}, \mathcal{I}^{\bullet}\right)$ is acyclic since for any affine open subscheme $U \subset X$ the 
higher Ext spaces between the components of the restrictions of $p_{1}^{*} \mathcal{N}$ and $\Delta_{*} \mathcal{M}$ to $U \times_{k} U$ vanish. The latter assertion follows from the adjunction of derived functors $\mathbb{L} \Delta^{*}$ and $\Delta_{*}=\mathbb{R} \Delta_{*}$ or $p_{1}^{*}=\mathbb{L} p_{1}^{*}$ and $\mathbb{R} p_{1 *}$ together with the agreement of the derived direct/inverse images of (complexes of) quasicoherent sheaves with the compositions of morphisms of separated Noetherian schemes.

It remains to show that our complex will stay acyclic after applying the direct image functor $p_{2 *}$. According to the argument in the proof of Lemma B.2.3, the components of the matrix factorizations $\mathcal{H o m}_{X \times_{k} X \text {-qc }}\left(p_{1}^{*} \mathcal{N}, \mathcal{I}^{i}\right)$ are acyclic for the direct image; so are the components of the matrix factorization $\mathcal{H o m}_{X \times{ }_{k} X \text {-qc }}\left(p_{1}^{*} \mathcal{N}, \Delta_{*} \mathcal{M}\right)$, in view of the above local argument and since for any affine open subscheme $U \subset X$, the higher Ext spaces between the components of the restrictions of $p_{1}^{*} \mathcal{N}$ and $\Delta_{*} \mathcal{M}$ to $X \times_{k} U$ vanish. The latter assertion is checked in the same way as above.

Finally, the claim that the matrix factorization in question is injective follows from the computation in part (b), which shows that the left-hand side is an exact functor of the argument $\mathcal{K}$, because the right-hand side is.

Part (b) is straightforward:

$$
\begin{aligned}
\operatorname{Hom}_{\left(X, \mathcal{O}, w^{\prime \prime}\right)-\mathrm{qcoh}}\left(\mathcal{K}, p_{2 *} \mathcal{H o m} \times_{\times_{k} X-\mathrm{qc}}\left(p_{1}^{*} \mathcal{N}, \mathcal{J}\right)\right) \\
\simeq \operatorname{Hom}_{\left(X \times_{k} X, \mathcal{O}, w_{2}^{\prime \prime}\right)-\mathrm{qcoh}}\left(p_{2}^{*} \mathcal{K}, \mathcal{H o m} \times_{\times_{k} X-\mathrm{qc}}\left(p_{1}^{*} \mathcal{N}, \mathcal{J}\right)\right) \\
\simeq \operatorname{Hom}_{\left(X \times_{k} X, \mathcal{O}, w_{1}^{\prime}+w_{2}^{\prime \prime}\right)-\mathrm{qcoh}}\left(p_{1}^{*} \mathcal{N} \otimes_{\mathcal{O}_{X \times} X} p_{2}^{*} \mathcal{K}, \mathcal{J}\right) \\
\quad \simeq \operatorname{Hom}_{\left(X \times_{k} X, \mathcal{O}, w_{1}^{\prime}+w_{2}^{\prime \prime}\right)-\mathrm{qcoh}}\left(\mathcal{N} \otimes_{k} \mathcal{K}, \mathcal{J}\right)
\end{aligned}
$$

B.2.4. Hochschild cohomology. Our goal in the rest of this appendix is to compute the Hochschild (co)homology of the DG-category $\mathrm{DG}^{\text {abs }}((X, \mathcal{O}, w)$-coh) corresponding to the triangulated category $\mathrm{D}^{\mathrm{abs}}((X, \mathcal{O}, w)$-coh). The word "corresponding" here means, first of all, that there is a natural equivalence of (triangulated) categories $H^{0} \mathrm{DG}^{\text {abs }}((X, \mathcal{O}, w)$-coh $) \simeq \mathrm{D}^{\text {abs }}((X, \mathcal{O}, w)$-coh) (see [Positselski 2011b, Section 1.2]).

As the absolute derived category is constructed from the homotopy category of matrix factorizations using the Verdier localization procedure, so the DG-category $\operatorname{DG}^{\text {abs }}((X, \mathcal{O}, w)$-coh $)$ is obtained by applying a DG-version of localization to the DG-category of coherent matrix factorizations $(X, \mathcal{O}, w)$-coh of the potential $w$ on the scheme $X$ (see Section 1.2). Several such localization procedures are known, leading to naturally quasiequivalent DG-categories. As the Hochschild (co)homology of DG-categories are preserved by quasiequivalences [Polishchuk and Positselski 2012, Sections 2.1 and 2.4], it is not very important which localization procedure to choose. To be specific, let us say that we prefer Drinfeld's localization [Drinfeld 2004]. Similarly one localizes the DG-category $(X, \mathcal{O}, w)$-qcoh and 
obtains a DG-category $\mathrm{DG}^{\mathrm{co}}((X, \mathcal{O}, w)$-qcoh) "corresponding" to the coderived category $\mathrm{D}^{\mathrm{co}}((X, \mathcal{O}, w)$-qcoh $)$.

Our method will naturally allow us to compute the Hochschild cohomology $H H^{*}\left(\mathrm{DG}^{\text {abs }}((X, \mathcal{O}, w)\right.$-coh $\left.)\right)$ together with its structure of an associative (in fact, supercommutative, but we will neither prove nor use this fact) $\mathbb{Z} / 2$-graded algebra over $k$. Similarly, the Hochschild homology $H_{*}\left(\mathrm{DG}^{\text {abs }}((X, \mathcal{O}, w)\right.$-coh $\left.)\right)$ will be computed together with its structure of a $\mathbb{Z} / 2$-graded module over the $\mathbb{Z} / 2$-graded associative algebra $H H^{*}\left(\mathrm{DG}^{\mathrm{abs}}((X, \mathcal{O}, w)\right.$-coh $\left.)\right)$.

Let $X$ be a separated scheme of finite type over a field $k$ and $w \in \mathcal{O}(X)$ be a global regular function. Assume that the morphism of schemes $w: X \rightarrow \mathbb{A}_{k}^{1}$ is flat. Consider the Cartesian square $X \times_{k} X$ and endow it with the potential (global function) $w_{2}-w_{1}=p_{2}^{*}(w)-p_{1}^{*}(w)$.

Any $\mathbb{Z} / 2$-graded complex of quasicoherent sheaves $\mathcal{K}^{\bullet}$ on $X$ can be viewed as a matrix factorization of the potential $0 \in \mathcal{O}(X)$. Furthermore, one can take its direct image $\Delta_{*} \mathcal{K} \bullet$ with respect to the diagonal embedding $\Delta: X \rightarrow X \times_{k} X$ and consider it as a quasicoherent matrix factorization of the potential $w_{2}-w_{1}$ on $X \times_{k} X$. Given a bounded $\mathbb{Z}$-graded complex of quasicoherent sheaves $\mathcal{K} \bullet$, one can associate a $\mathbb{Z} / 2$-complex with it (by taking direct sums of all terms with the same parity) and then apply the above constructions.

Theorem B.2.4. Assume that there exists a closed subscheme $Z \subset X$ such that $\left.w\right|_{Z}=0$, the function $w$ is noncritical on $X \backslash Z$, and the scheme $Z$ admits a smooth stratification over $k$. In particular, if the field $k$ is perfect, it suffices to require that the function $w$ on $X$ have no critical values but zero (and take $Z=\{w=0\}$ ). Then there is a natural isomorphism between the Hochschild cohomology algebra $H H^{*}\left(\mathrm{DG}^{\mathrm{abs}}((X, \mathcal{O}, w)\right.$-coh $\left.)\right)$ and the Ext algebra

$$
\operatorname{Hom}_{D^{c o}\left(\left(X \times_{k} X, \mathcal{O}, w_{2}-w_{1}\right)-\text { qcoh }\right)}\left(\Delta_{*} \mathcal{D}_{X}^{\bullet}, \Delta_{*} \mathcal{D}_{X}^{\bullet}[*]\right),
$$

where $\mathcal{D}_{X}^{\bullet}$ denotes a dualizing complex on $X$.

Proof. By the definition, the Hochschild cohomology algebra of a $\mathbb{Z} / 2$-graded DG-category $D G$ is the $\mathbb{Z} / 2$-graded algebra $\operatorname{Hom}_{\mathrm{D}\left(\mathrm{DG} \otimes_{k} \mathrm{DG}^{\text {op }} \text {-mod }\right)}(\mathrm{DG}, \mathrm{DG}[*])$, where the Hom is taken in the conventional derived category $D\left(D G \otimes_{k} D^{\circ{ }^{o p}}\right.$-mod) of DG-bimodules over DG (or DG-modules over DG $\otimes_{k}$ DG ${ }^{\circ}$ ) between two copies of the diagonal DG-bimodule DG over DG [Polishchuk and Positselski 2012, Sections 2.4 and 3.1].

Specializing to the case of the DG-category $D_{w}=D^{\text {abs }}((X, \mathcal{O}, w)$-coh $)$, we notice, first of all, that the contravariant Serre-Grothendieck duality functor $\mathcal{M} \longmapsto \mathcal{H o m}_{\mathcal{O}_{X}}\left(\mathcal{M}, \mathcal{D}_{X}^{\bullet}\right)$ (see Section 2.5) provides a quasiequivalence between the DG-categories $\mathrm{DG}^{\mathrm{abs}}((X, \mathcal{O}, w) \text {-coh })^{\mathrm{op}}$ and $\mathrm{DG}^{\mathrm{abs}}((X, \mathcal{O},-w)$-coh). Furthermore, 
the external tensor product is a DG-functor

$$
\begin{aligned}
\operatorname{DG}^{\text {abs }}((X, \mathcal{O},-w)-\mathrm{coh}) \otimes_{k} \mathrm{DG}^{\mathrm{abs}} & ((X, \mathcal{O}, w)-\mathrm{coh}) \\
& \longrightarrow \mathrm{DG}^{\mathrm{abs}}\left(\left(X \times_{k} X, \mathcal{O}, w_{2}-w_{1}\right)-\mathrm{coh}\right),
\end{aligned}
$$

which, according to Proposition B.2.2 and Theorem B.2.2, induces an equivalence between the derived categories of (left or right) DG-modules over the two DG-categories in the left-hand and right-hand sides. Composing the SerreGrothendieck duality with the external tensor product, we obtain (perhaps, after replacing our DG-categories with naturally quasiequivalent ones) a DG-functor

$$
\begin{aligned}
\operatorname{DG}^{\text {abs }}((X, \mathcal{O}, w)-c o h)^{\mathrm{op}} \otimes_{k} \mathrm{DG}^{\text {abs }}( & (X, \mathcal{O}, w) \text {-coh }) \\
& \longrightarrow \mathrm{DG}^{\text {abs }}\left(\left(X \times_{k} X, \mathcal{O}, w_{2}-w_{1}\right)-\mathrm{coh}\right)
\end{aligned}
$$

having the same property with respect to the derived categories of DG-modules over the left-hand and right-hand sides as the DG-functor (28).

We are interested specifically in the diagonal right DG-module over $D G_{w}^{o p} \otimes_{k} D G_{w}$, that is, the contravariant functor from $\mathrm{DG}_{w}^{o p} \otimes_{k} \mathrm{DG}_{w}$ to the DG-category of $\mathbb{Z} / 2$ graded complexes of $k$-vector spaces taking an object $\left(\mathcal{M}^{\text {op }}, \mathcal{K}\right)$ to the complex $\operatorname{Hom}_{D G}(\mathcal{K}, \mathcal{M})$. It is claimed that the diagonal DG-module is naturally quasiisomorphic to the DG-module obtained by composing the DG-functor (29) with the right DG-module over the right-hand side represented by the object

$$
\Delta_{*} \mathcal{D}_{X}^{\bullet} \in \mathrm{DG}^{\mathrm{abs}}\left(\left(X \times_{k} X, \mathcal{O}, w_{2}-w_{1}\right)-\mathrm{coh}\right) \subset \mathrm{DG}^{\mathrm{co}}\left(\left(X \times_{k} X, \mathcal{O}, w_{2}-w_{1}\right) \text {-qcoh }\right) \text {. }
$$

Indeed, for any quasicoherent matrix factorizations $\mathcal{K}$ and $\mathcal{N}$ of the potentials $w$ and $-w$ on $X$ there is a natural isomorphism of $\mathbb{Z} / 2$-graded complexes of abelian groups (see (27))

$$
\begin{aligned}
\operatorname{Hom}_{(X, \mathcal{O}, w)-q \operatorname{coh}}\left(\mathcal{K}, \mathcal{H o m}_{\mathcal{O}_{X}}(\mathcal{N},\right. & \left.\left.\mathcal{D}_{X}^{\bullet}\right)\right) \\
& \simeq \operatorname{Hom}_{\left(X \times_{k} X, \mathcal{O}, w_{2}-w_{1}\right) \text {-qcoh }}\left(\mathcal{N} \otimes_{k} \mathcal{K}, \Delta_{*} \mathcal{D}_{X}^{\bullet}\right) .
\end{aligned}
$$

Proposition B.2.3 shows how one can pass from this isomorphism to a quasi-isomorphism of the similar complexes of morphisms in the DG-categories $\operatorname{DG}^{\mathrm{co}}((X, \mathcal{O}, w)$ -qcoh) and $\mathrm{DG}^{\mathrm{co}}\left(\left(X \times_{k} X, \mathcal{O}, w_{2}-w_{1}\right)\right.$-qcoh $)$.

Now morphisms between representable DG-modules in the derived category of DG-modules over a DG-category DG are computed by the complex of morphisms in DG between the representing objects, so our proof is finished.

Remark B.2.4. Given a separated scheme $X$ of finite type over a field $k$, let $\mathcal{D}_{X}^{\bullet}$ be a dualizing complex on $X$ and $\mathcal{D}_{X \times_{k} X}^{\bullet}$ be a dualizing complex on $X \times_{k} X$ such 
that $\mathcal{D}_{X}^{\bullet} \simeq \mathbb{R} \Delta^{!}\left(\mathcal{D}_{X \times{ }_{k} X}^{\bullet}\right)$. Then the antiequivalence of absolute derived categories

$$
\begin{aligned}
\mathcal{H o m}_{X \times_{k} X \text {-qc }}\left(-, \mathcal{D}_{X \times_{k} X}^{\bullet}\right): \mathrm{D}^{\mathrm{abs}}\left(\left(X \times_{k} X,\right.\right. & \left.\left.\mathcal{O}, w_{1}-w_{2}\right) \text {-coh }\right)^{\text {op }} \\
& \simeq \mathrm{D}^{\mathrm{abs}}\left(\left(X \times_{k} X, \mathcal{O}, w_{2}-w_{1}\right) \text {-coh }\right)
\end{aligned}
$$

from Proposition 2.5 transforms the object $\Delta_{*} \mathcal{O}_{X} \in \mathrm{D}^{\mathrm{abs}}\left(\left(X \times_{k} X, \mathcal{O}, w_{1}-w_{2}\right)\right.$-coh $)$ into the object $\Delta_{*} \mathcal{D}_{X}^{\bullet} \in \mathrm{D}^{\mathrm{abs}}\left(\left(X \times_{k} X, \mathcal{O}, w_{2}-w_{1}\right)\right.$-coh) (see Proposition 3.7). Therefore, in the assumptions of Theorem B.2.4, the Hochschild cohomology algebra $H H^{*}\left(\mathrm{D}^{\text {abs }}((X, \mathcal{O}, w)\right.$-coh $\left.)\right)$ of the DG-category $\mathrm{DG}^{\text {abs }}((X, \mathcal{O}, w)$-coh) can be also identified with the Ext algebra

$$
\operatorname{Hom}_{\mathrm{D}^{\mathrm{abs}}\left(\left(X \times_{k} X, \mathcal{O}, w_{1}-w_{2}\right) \text {-coh }\right)}\left(\Delta_{*} \mathcal{O}_{X}, \Delta_{*} \mathcal{O}_{X}[*]\right)^{\mathrm{op}}
$$

(cf. Remark B.2.7 below).

B.2.5. Cotensor product of complexes of quasicoherent sheaves. Let $X$ be a separated Noetherian scheme. Then there is a tensor product functor on the coderived category of (ZZ-graded complexes of) flat quasicoherent sheaves on $X$ (cf. [Murfet 2007, Chapter 6])

$$
\otimes_{\mathcal{O}_{X}}: \mathrm{D}^{\mathrm{co}}\left(X-\mathrm{q} \operatorname{coh}_{\mathrm{fl}}\right) \times \mathrm{D}^{\mathrm{co}}\left(X-\mathrm{q} \operatorname{coh}_{\mathrm{fl}}\right) \longrightarrow \mathrm{D}^{\mathrm{co}}\left(X-\mathrm{q} \operatorname{coh}_{\mathrm{fl}}\right),
$$

and a similar functor of the tensor product on the coderived categories of flat and arbitrary quasicoherent sheaves (see [Positselski 2012, Section 4.12])

$$
\otimes_{\mathcal{O}_{X}}: \mathrm{D}^{\mathrm{co}}\left(X \text {-qcoh }_{\mathrm{fl}}\right) \times \mathrm{D}^{\mathrm{co}}(X \text {-qcoh }) \longrightarrow \mathrm{D}^{\mathrm{co}}(X \text {-qcoh }) .
$$

Now let $\mathcal{D}_{X}^{\bullet}$ be a dualizing complex on $X$ (viewed, as usual, as a finite complex of injective quasicoherent sheaves). Then the equivalence of triangulated categories $\mathrm{D}^{\mathrm{co}}\left(X\right.$-qcoh $\left._{\mathrm{fl}}\right) \simeq \mathrm{D}^{\mathrm{co}}(X$-qcoh $)$ constructed using the dualizing complex $\mathcal{D}_{X}^{\bullet}$ (see [Murfet 2007, Chapter 8]) transforms the tensor product functor (30) into the tensor product functor (31). One can use the same equivalence of categories to define a tensor triangulated category structure with the unit object $\mathcal{D}_{X}^{\bullet}$ on the coderived category $\mathrm{D}^{\mathrm{co}}(X$-qcoh). We call this operation the cotensor product of complexes of quasicoherent sheaves on $X$ and denote it by

$$
\square_{\mathcal{D}_{X}^{\bullet}}: \mathrm{D}^{\mathrm{co}}(X \text {-qcoh }) \times \mathrm{D}^{\mathrm{co}}(X \text {-qcoh }) \longrightarrow \mathrm{D}^{\mathrm{co}}(X \text {-qcoh }) .
$$

Explicitly, $\mathcal{N}^{\bullet} \square_{\mathcal{D}_{X}^{\bullet}} \mathcal{M}^{\bullet}=\mathcal{D}_{X}^{\bullet} \otimes_{\mathcal{O}_{X}} \mathcal{H o m}{ }_{X \text {-qc }}\left(\mathcal{D}_{X}^{\bullet}, \mathcal{N}^{\bullet}\right) \otimes_{\mathcal{O}_{X}} \mathcal{H o m}{ }_{X \text {-qc }}\left(\mathcal{D}_{X}^{\bullet}, \mathcal{M}^{\bullet}\right)$ for any complexes of injective quasicoherent sheaves $\mathcal{N}^{\bullet}$ and $\mathcal{M}^{\bullet}$ on $X$ (cf. Lemma 1.7(b)) and also $\mathcal{N}^{\bullet} \square_{\mathcal{D}_{X}^{\bullet}} \mathcal{M}^{\bullet}=\mathcal{H o m}_{X \text {-qc }}\left(\mathcal{D}_{X}^{\bullet}, \mathcal{N}^{\bullet}\right) \otimes_{\mathcal{O}_{X}} \mathcal{M}^{\bullet}$ for any complex of injective quasicoherent sheaves $\mathcal{N}^{\bullet}$ and any complex of quasicoherent sheaves $\mathcal{M}^{\bullet}$ on $X$.

Recall that the full triangulated subcategory of bounded-below complexes in $\mathrm{D}^{\mathrm{co}}\left(X\right.$-qcoh) is equivalent to $\mathrm{D}^{+}(X$-qcoh) (see [Positselski 2010, Lemma 2.1 and 
Remark 4.1] or [Positselski 2012, Lemma A.1.2]). Denote by $\mathrm{D}_{\text {coh }}^{+}(X$-qcoh) the full triangulated subcategory in $\mathrm{D}^{+}(X$-qcoh) consisting of complexes with coherent cohomology sheaves; then the category $\mathrm{D}_{\text {coh }}^{+}(X$-qcoh $)$ can be also viewed as a full triangulated subcategory in $\mathrm{D}^{\mathrm{co}}(X$-qcoh $)$.

For any complexes of quasicoherent sheaves $\mathcal{N}^{\bullet}$ and $\mathcal{M}^{\bullet}$ on $X$ there is a natural morphism of complexes of quasicoherent sheaves

$$
\begin{aligned}
\mathcal{D}_{X}^{\bullet} \otimes_{\mathcal{O}_{X}} & \mathcal{H o m}_{X \text {-qc }}\left(\mathcal{D}_{X}^{\bullet}, \mathcal{N}^{\bullet}\right) \otimes_{\mathcal{O}_{X}} \mathcal{H o m}_{X \text {-qc }}\left(\mathcal{D}_{X}^{\bullet}, \mathcal{M}^{\bullet}\right) \\
& \longrightarrow \mathcal{H o m}_{X \text {-qc }}\left(\mathcal{H o m}{ }_{X \text {-qc }}\left(\mathcal{N}^{\bullet}, \mathcal{D}_{X}^{\bullet}\right) \otimes_{\mathcal{O}_{X}} \mathcal{H o m}_{X \text {-qc }}\left(\mathcal{M}^{\bullet}, \mathcal{D}_{X}^{\bullet}\right), \mathcal{D}_{X}^{\bullet}\right)
\end{aligned}
$$

on $X$ defined in terms of the composition morphisms

$$
\mathcal{H o m}_{X \text {-qc }}\left(\mathcal{D}_{X}^{\bullet}, \mathcal{K}^{\bullet}\right) \otimes_{\mathcal{O}_{X}} \mathcal{H o m}_{X \text {-qc }}\left(\mathcal{K}^{\bullet}, \mathcal{D}_{X}^{\bullet}\right) \longrightarrow \mathcal{H o m}_{X \text {-qc }}\left(\mathcal{D}_{X}^{\bullet}, \mathcal{D}_{X}^{\bullet}\right)
$$

for complexes of quasicoherent sheaves $\mathcal{K}^{\bullet}$ on $X$ and the natural quasi-isomorphism

$$
\mathcal{D}_{X}^{\bullet} \otimes_{\mathcal{O}_{X}} \operatorname{Hom}_{X \text {-qc }}\left(\mathcal{D}_{X}^{\bullet}, \mathcal{D}_{X}^{\bullet}\right) \otimes_{\mathcal{O}_{X}} \mathcal{H o m}{ }_{X \text {-qc }}\left(\mathcal{D}_{X}^{\bullet}, \mathcal{D}_{X}^{\bullet}\right) \longrightarrow \mathcal{D}_{X}^{\bullet} .
$$

Theorem B.2.5. For any bounded-below complexes of injective quasicoherent sheaves $\mathcal{N}^{\bullet}$ and $\mathcal{M}^{\bullet}$ with coherent cohomology sheaves on a separated Noetherian scheme $X$ with a dualizing complex $\mathcal{D}_{X}^{\bullet}$, the natural morphism (33) is a homotopy equivalence of bounded-below complexes of injective quasicoherent sheaves on $X$ with coherent cohomology sheaves.

Proof. By Lemma 2.5(b) and (c), both sides of (33) are bounded-below complexes of injective quasicoherent sheaves. Since the functor

$$
\mathcal{H o m}_{X \text {-qc }}\left(-, \mathcal{D}_{X}^{\bullet}\right): \mathrm{D}(X \text {-qcoh }) \longrightarrow \mathrm{D}(X \text {-qcoh })
$$

takes $\mathrm{D}_{\text {coh }}^{+}(X$-qcoh $) \subset \mathrm{D}^{+}(X$-qcoh $)$ into $\mathrm{D}^{-}(X$-coh $) \subset \mathrm{D}^{-}(X$-qcoh $)$ and vice versa, while the derived tensor product functor

$$
\otimes_{X}^{\llbracket}: \mathrm{D}^{-}(X \text {-qcoh }) \times \mathrm{D}^{-}(X \text {-qcoh }) \longrightarrow \mathrm{D}^{-}(X \text {-qcoh })
$$

takes $\mathrm{D}^{-}(X$-coh $) \times \mathrm{D}^{-}(X$-coh $)$ into $\mathrm{D}^{-}(X$-coh $)$, the right-hand side has coherent cohomology sheaves.

It remains to prove the homotopy equivalence claim. Since the homotopy category of bounded-below complexes of injectives is equivalent to $\mathrm{D}^{+}(X$-qcoh), one only has to check that the map is a quasi-isomorphism. Let us first show that it suffices to do so for complexes of sheaves on affine open subschemes $U \subset X$.

Indeed, for any quasicoherent sheaves $\mathcal{E}$ and $\mathcal{K}$ on $X$ there is a natural morphism of quasicoherent sheaves $\left.\mathcal{H o m}_{X \text {-qc }}(\mathcal{E}, \mathcal{K})\right|_{U} \rightarrow \mathcal{H o m}_{U \text {-qc }}\left(\left.\mathcal{E}\right|_{U},\left.\mathcal{K}\right|_{U}\right)$ on $U$. The morphism of complexes of quasicoherent sheaves $\left.\mathcal{H o m}_{X \text {-qc }}\left(\mathcal{E}^{\bullet}, \mathcal{K}^{\bullet}\right)\right|_{U} \rightarrow$ 
$\mathcal{H o m}_{U \text {-qc }}\left(\left.\mathcal{E}^{\bullet}\right|_{U},\left.\mathcal{K}^{\bullet}\right|_{U}\right)$ is a quasi-isomorphism whenever the complex $\mathcal{E}^{\bullet}$ has coherent cohomology, $\mathcal{K}^{\bullet}$ is a complex of injective quasicoherent sheaves, and one of the complexes $\mathcal{E}^{\bullet}$ and $\mathcal{K}^{\bullet}$ is finite.

Finally, the tensor product in the right-hand side preserves quasi-isomorphisms of bounded-above complexes of flat quasicoherent sheaves, while the one in the left-hand side is well-defined on the coderived category of (complexes of) flat quasicoherent sheaves. It remains to notice that the functor $\operatorname{Hom}_{X \text {-qc }}\left(\mathcal{D}_{X}^{\bullet},-\right)$ in the equivalence of categories in Theorem 2.5 agrees with the restrictions to open subschemes since so does its inverse functor $\mathcal{D}_{X}^{\bullet} \otimes_{\mathcal{O}_{X}}-$.

Now that we are on an affine scheme $U$, pick bounded-above complexes of vector bundles ' $\mathcal{N} \bullet$ and ' $\mathcal{M} \bullet$ isomorphic to $\mathcal{H o m}_{U \text {-qc }}\left(\mathcal{N}^{\bullet}, \mathcal{D}_{U}^{\bullet}\right)$ and $\mathcal{H o m}_{U \text {-qc }}\left(\mathcal{M}^{\bullet}, \mathcal{D}_{U}^{\bullet}\right)$, respectively, in $\mathrm{D}^{-}(U$-coh $)$. Given the isomorphisms

$$
\begin{aligned}
\mathcal{H o m}_{U \text {-qc }}\left(\mathcal{D}_{U}^{\bullet}, \mathcal{H o m}_{U \text {-qc }}\left({ }^{\prime} \mathcal{N}^{\bullet}, \mathcal{D}_{U}^{\bullet}\right)\right) \simeq \mathcal{H o m}_{U-\mathrm{qc}}\left({ }^{\prime} \mathcal{N}^{\bullet}, \mathcal{H o m}_{U \text {-qc }}\left(\mathcal{D}_{U}^{\bullet}, \mathcal{D}_{U}^{\bullet}\right)\right) \\
\simeq \mathcal{H} o m_{U-\mathrm{qc}}\left({ }^{\prime} \mathcal{N}^{\bullet}, \mathcal{O}_{U}\right),
\end{aligned}
$$

and similar isomorphisms for $\mathcal{M}^{\bullet}$ in $\mathrm{D}^{\mathrm{co}}\left(U-\mathrm{q} \mathrm{coh}_{\mathrm{fl}}\right)$, the assertion reduces to the obvious isomorphism of complexes

$$
\begin{aligned}
\mathcal{D}_{X}^{\bullet} \otimes_{\mathcal{O}_{X}} \mathcal{H o m}_{U-\mathrm{qc}}\left({ }^{\prime} \mathcal{N}^{\bullet}, \mathcal{O}_{U}\right) \otimes_{\mathcal{O}_{U}} \mathcal{H o m}_{U-\mathrm{qc}}\left({ }^{\prime} \mathcal{M}^{\bullet}, \mathcal{O}_{U}\right) \\
\simeq \operatorname{Hom}_{U-\mathrm{qc}}\left({ }^{\prime} \mathcal{N}^{\bullet} \otimes_{\mathcal{O}_{X}}{ }^{\prime} \mathcal{M}^{\bullet}, \mathcal{D}_{U}^{\bullet}\right) .
\end{aligned}
$$

For any complexes of quasicoherent sheaves $\mathcal{K}^{\bullet}$ and $\mathcal{M}^{\bullet}$ on $X$ we denote by $\mathcal{H o m} \underset{X \text {-qc }}{\oplus}\left(\mathcal{K}^{\bullet}, \mathcal{M}^{\bullet}\right)$ the complex of quasicoherent sheaves on $X$ obtained by totalizing the bicomplex of quasicoherent internal Hom sheaves $\mathcal{H o m}_{X \text {-qc }}\left(\mathcal{K}^{i}, \mathcal{M}^{j}\right)$ by taking infinite direct sums along the diagonals. Assuming that $\mathcal{M}^{\bullet}$ is a complex of injective quasicoherent sheaves, the complex $\mathcal{H o m}_{X \text {-qc }}^{\oplus}\left(\mathcal{K}^{\bullet}, \mathcal{M}^{\bullet}\right)$ is absolutely acyclic with respect to $X$-qcoh whenever the complex $\mathcal{K}^{\bullet}$ is (see Lemma 2.5(a)).

In the same assumption, the complex $\mathcal{H o m}_{X \text {-qc }}^{\oplus}\left(\mathcal{K}^{\bullet}, \mathcal{M}^{\bullet}\right)$ is also coacyclic with respect to $X$-qcoh whenever the complex of quasicoherent sheaves $\mathcal{K}^{\bullet}$ is acyclic and bounded from above [Positselski 2010, Lemma 2.1]. Therefore, representing the second argument of $\mathcal{H o m}_{X \text {-qc }}^{\oplus}$ by complexes of injectives, one can construct the right derived functors

$$
\mathbb{R} \mathcal{H} o m_{X \text {-qc }}^{\oplus}: D^{\text {abs }}(X \text {-qcoh })^{o p} \times D^{c o}(X \text {-qcoh }) \longrightarrow D^{\text {abs }}(X \text {-qcoh })
$$

and

$$
\mathbb{R} \mathcal{H} \text { om }{ }_{X \text {-qc }}^{\oplus}: \mathrm{D}^{-}(X \text {-qcoh })^{\mathrm{op}} \times \mathrm{D}^{\mathrm{co}}(X \text {-qcoh }) \longrightarrow \mathrm{D}^{\mathrm{co}}(X \text {-qcoh })
$$

of the functor $\mathcal{H o m} \underset{X \text {-qc }}{\oplus}$. 
For any complexes of quasicoherent sheaves $\mathcal{N}^{\bullet}$ and $\mathcal{M}^{\bullet}$ on $X$ there is a natural morphism of complexes of quasicoherent sheaves

$$
\mathcal{N}^{\bullet} \otimes_{\mathcal{O}_{X}} \mathcal{H o m}_{X \text {-qc }}\left(\mathcal{D}_{X}^{\bullet}, \mathcal{M}^{\bullet}\right) \longrightarrow \mathcal{H o m}_{X \text {-qc }}^{\oplus}\left(\mathcal{H o m}_{X \text {-qc }}\left(\mathcal{N}^{\bullet}, \mathcal{D}_{X}^{\bullet}\right), \mathcal{M}^{\bullet}\right)
$$

on $X$ defined in terms of the composition/evaluation morphism

$$
\mathcal{N}^{\bullet} \otimes_{\mathcal{O}_{X}} \mathcal{H o m}_{X \text {-qc }}\left(\mathcal{N}^{\bullet}, \mathcal{D}_{X}^{\bullet}\right) \otimes_{\mathcal{O}_{X}} \mathcal{H o m}_{X \text {-qc }}\left(\mathcal{D}_{X}^{\bullet}, \mathcal{M}^{\bullet}\right) \longrightarrow \mathcal{M}^{\bullet}
$$

Proposition B.2.5. For any bounded-below complex of injective quasicoherent sheaves $\mathcal{N}^{\bullet}$ with coherent cohomology sheaves and any complex of injective quasicoherent sheaves $\mathcal{M}^{\bullet}$ on $X$, the natural morphism (36) is a homotopy equivalence of complexes of injective quasicoherent sheaves on $X$.

Proof. It suffices to check that the morphism (36) is an isomorphism in $\mathrm{D}^{\mathrm{co}}(X$-qcoh). Since both sides of the desired isomorphism are well-defined as functors of the argument $\mathcal{N}^{\bullet} \in \mathrm{D}^{+}(X$-qcoh $)$ taking values in $\mathrm{D}^{\text {co }}(X$-qcoh $)$, one can freely replace $\mathcal{N}^{\bullet}$ with any quasi-isomorphic bounded-below complex of quasicoherent sheaves. The same applies to the bounded-above complex $\mathcal{H o m}_{X \text {-qc }}\left(\mathcal{N}^{\bullet}, \mathcal{D}_{X}^{\bullet}\right)$ in the right-hand side of (36).

Since all the functors involved are local in $X$ up to isomorphism in the relevant triangulated categories, it suffices to consider complexes of sheaves over affine open subschemes $U \subset X$ (see Remark 1.3). Representing the object $\mathcal{H o m}_{U \text {-qc }}\left(\mathcal{N}^{\bullet}, \mathcal{D}_{U}^{\bullet}\right) \in$ $\mathrm{D}^{-}(U$-coh $) \subset \mathrm{D}^{-}(U$-qcoh $)$ by a bounded-above complex of vector bundles ${ }^{\prime} \mathcal{N}^{\bullet}$, it remains to notice the isomorphism of complexes

$$
\mathcal{H o m}_{U \text {-qc }}\left({ }^{\prime} \mathcal{N}^{\bullet}, \mathcal{D}_{U}^{\bullet}\right) \otimes_{\mathcal{O}_{U}} \mathcal{F}^{\bullet} \simeq \mathcal{H o m}_{U \text {-qc }}^{\oplus}\left(\mathcal{N}^{\bullet}, \mathcal{D}_{U}^{\bullet} \otimes_{\mathcal{O}_{U}} \mathcal{F}^{\bullet}\right)
$$

for any complex of quasicoherent sheaves $\mathcal{F}^{\bullet}$ on $U$ and point out that the functor $\mathcal{H o m}_{U \text {-qc }}^{\oplus}\left({ }^{\prime} \mathcal{N}^{\bullet},-\right)$ takes a homotopy equivalence

$$
\mathcal{D}_{U}^{\bullet} \otimes_{\mathcal{O}_{U}} \mathcal{H o m}_{U-\mathrm{qc}}\left(\mathcal{D}_{U}^{\bullet}, \mathcal{M}^{\bullet}\right) \longrightarrow \mathcal{M}^{\bullet}
$$

to a homotopy equivalence.

In the particular cases when either $\mathcal{N}^{\bullet}$ is a finite complex of quasicoherent sheaves with coherent cohomology sheaves, or $\mathcal{N}^{\bullet}$ is a bounded-below complex of quasicoherent sheaves with coherent cohomology sheaves and $\mathcal{M}^{\bullet}$ is a boundedbelow complex of quasicoherent sheaves, the direct sum totalization of the bicomplex $\mathcal{H o m}_{X \text {-qc }}$ in the right-hand side of the isomorphism (36) in the coderived category $\mathrm{D}^{\mathrm{co}}(X$-qcoh $)$ is no different from the conventional direct product totalization.

Finally, let $X$ be a separated scheme of finite type over a field $k$ and $\pi: X \rightarrow \operatorname{Spec} k$ be its structure morphism. Then $\mathcal{D}_{X}^{\bullet} \simeq \pi^{+} \mathcal{O}_{\text {Spec } k}$ (see Section 3.7) is a natural choice of the dualizing complex on $X$. Let $\pi \times_{k} \pi: X \times_{k} X \rightarrow$ Spec $k$ be the structure morphism of the Cartesian square of $X$ over $k$. Then the dualizing complex 
$\mathcal{D}_{X \times_{k} X}^{\bullet}=\left(\pi \times_{k} \pi\right)^{+} \mathcal{O}_{\text {Spec } k}$ on $X \times_{k} X$ is quasi-isomorphic to the external tensor product $\mathcal{D}_{X}^{\bullet} \otimes_{k} \mathcal{D}_{X}^{\bullet}$, and one has $\mathcal{D}_{X}^{\bullet} \simeq \Delta^{+}\left(\mathcal{D}_{X \times_{k} X}^{\bullet}\right) \simeq \mathbb{R} \Delta^{!}\left(\mathcal{D}_{X}^{\bullet} \otimes_{k} \mathcal{D}_{X}^{\bullet}\right)$, where $\Delta: X \rightarrow X \times_{k} X$ denotes the diagonal map.

The equivalence of categories $\mathrm{D}^{\mathrm{co}}\left(X \times_{k} X\right.$-qcoh $\left.{ }_{\mathrm{fl}}\right) \simeq \mathrm{D}^{\mathrm{co}}\left(X \times_{k} X\right.$-qcoh $)$ constructed using the dualizing complex $\mathcal{D}_{X \times_{k} X}^{\bullet}$ and the similar equivalence $\mathrm{D}^{\mathrm{co}}\left(X\right.$-qcoh $\left.\mathrm{fl}_{\mathrm{fl}}\right) \simeq$ $\mathrm{D}^{\mathrm{co}}(X$-qcoh $)$ constructed using the dualizing complex $\mathcal{D}_{X}^{\bullet}$ transform the external tensor product functor

$$
\otimes_{k}: \mathrm{D}^{\mathrm{co}}\left(X-\mathrm{qcoh}_{\mathrm{fl}}\right) \times \mathrm{D}^{\mathrm{co}}\left(X-\mathrm{qcoh} \mathrm{fl}_{\mathrm{fl}}\right) \longrightarrow \mathrm{D}^{\mathrm{co}}\left(X \times_{k} X-\mathrm{qcoh}_{\mathrm{fl}}\right)
$$

into the external tensor product functor

$$
\otimes_{k}: \mathrm{D}^{\mathrm{co}}(X \text {-qcoh }) \times \mathrm{D}^{\mathrm{co}}(X \text {-qcoh }) \longrightarrow \mathrm{D}^{\mathrm{co}}\left(X \times_{k} X \text {-qcoh }\right)
$$

since so do the functors $\mathcal{D}_{X}^{\bullet} \otimes_{\mathcal{O}_{X}}-$ and $\mathcal{D}_{X \times_{k} X}^{\bullet} \otimes_{\mathcal{O}_{X \times k} X}-$.

Let $\mathcal{N}^{\bullet}$ and $\mathcal{M}^{\bullet}$ be two complexes of injective quasicoherent sheaves on $X$, and let $\mathcal{J}^{\bullet}$ be a complex of injective quasicoherent sheaves on $X \times_{k} X$ isomorphic to $\mathcal{N}^{\bullet} \otimes_{k} \mathcal{M}^{\bullet}$ in $\mathrm{D}^{\mathrm{co}}\left(X \times_{k} X\right.$-qcoh $)$. Then in the coderived categories of quasicoherent sheaves one has

$$
\begin{aligned}
\mathcal{N}^{\bullet} \square_{\mathcal{D}_{X}^{\bullet}} \mathcal{M}^{\bullet}=\mathcal{D}_{X}^{\bullet} \otimes_{\mathcal{O}_{X}} \Delta^{*}\left(\mathcal{H o m}_{\mathcal{O}_{X}}\left(\mathcal{D}_{X}^{\bullet}, \mathcal{N}^{\bullet}\right) \otimes_{k} \mathcal{H o m}_{\mathcal{O}_{X}}\left(\mathcal{D}_{X}^{\bullet}, \mathcal{M}^{\bullet}\right)\right) \\
\simeq \mathcal{D}_{X}^{\bullet} \otimes_{\mathcal{O}_{X}} \Delta^{*} \mathcal{H o m}{\mathcal{O}_{X}}\left(\mathcal{D}_{X \times_{k} X}^{\bullet}, \mathcal{J}^{\bullet}\right) \simeq \mathbb{R} \Delta^{!}\left(\mathcal{N}^{\bullet} \otimes_{k} \mathcal{M}^{\bullet}\right)
\end{aligned}
$$

by [Positselski 2012, Theorem 5.15.3] applied to the proper morphism (actually, closed embedding) $\Delta$. We have obtained the formula

$$
\mathcal{N}^{\bullet} \square_{\pi}+\mathcal{O}_{\text {Spec } k} \mathcal{M}^{\bullet} \simeq \mathbb{R} \Delta^{!}\left(\mathcal{N}^{\bullet} \otimes_{k} \mathcal{M}^{\bullet}\right)
$$

for the cotensor product of complexes of quasicoherent sheaves on the scheme $X$ (see the end of Section 1.8 for the notation $\mathbb{R} f !$ as applied to objects of the coderived category of quasicoherent sheaves).

B.2.6. Cotensor product of matrix factorizations. The equivalences of triangulated categories

$$
\begin{aligned}
\mathrm{D}^{\mathrm{co}}\left(\left(X, \mathcal{L}, w^{\prime \prime}\right)-\mathrm{qcoh}_{\mathrm{fl}}\right) & \simeq \mathrm{D}^{\mathrm{co}}\left(\left(X, \mathcal{L}, w^{\prime \prime}\right)-\mathrm{qcoh}\right) \\
\mathrm{D}^{\mathrm{co}}\left(\left(X, \mathcal{L}, w^{\prime}+w^{\prime \prime}\right)-\mathrm{qcoh}_{\mathrm{fl}}\right) & \simeq \mathrm{D}^{\mathrm{co}}\left(\left(X, \mathcal{L}, w^{\prime}+w^{\prime \prime}\right)-\mathrm{qcoh}\right)
\end{aligned}
$$

constructed using a dualizing complex $\mathcal{D}_{X}^{\bullet}$ (see Section 2.5) transform the tensor product functor (14) into the tensor product functor (15). So one can use the same equivalences of categories together with the similar equivalence

$$
\mathrm{D}^{\mathrm{co}}\left(\left(X, \mathcal{L}, w^{\prime}\right)-\mathrm{qcoh}{ }_{\mathrm{fl}}\right) \simeq \mathrm{D}^{\mathrm{co}}\left(\left(X, \mathcal{L}, w^{\prime}\right)-\mathrm{qcoh}\right)
$$


(constructed using the same dualizing complex $\mathcal{D}_{X}^{\bullet}$ ) in order to define a triangulated functor of two arguments

$$
\begin{aligned}
\square_{\mathcal{D}_{X}^{\bullet}}: \mathrm{D}^{\mathrm{co}}\left(\left(X, \mathcal{L}, w^{\prime}\right)-\mathrm{qcoh}\right) \times \mathrm{D}^{\mathrm{co}}\left(\left(X, \mathcal{L}, w^{\prime \prime}\right) \text {-qcoh }\right) & \\
\longrightarrow & \mathrm{D}^{\mathrm{co}}\left(\left(X, \mathcal{L}, w^{\prime}+w^{\prime \prime}\right)-\mathrm{qcoh}\right),
\end{aligned}
$$

which we call the cotensor product of matrix factorizations.

As in the case of complexes of quasicoherent sheaves, one explicitly has

$$
\mathcal{N} \square_{\mathcal{D}_{X}^{\bullet}} \mathcal{M}=\mathcal{D}_{X}^{\bullet} \otimes_{\mathcal{O}_{X}} \mathcal{H o m}_{X \text {-qc }}\left(\mathcal{D}_{X}^{\bullet}, \mathcal{N}\right) \otimes_{\mathcal{O}_{X}} \mathcal{H o m}_{X \text {-qc }}\left(\mathcal{D}_{X}^{\bullet}, \mathcal{M}\right)
$$

for any injective quasicoherent matrix factorizations $\mathcal{N}$ and $\mathcal{M}$ on $X$, and also

$$
\mathcal{N} \square_{\mathcal{D}_{X}^{\bullet}} \mathcal{M}=\mathcal{H o m}_{X \text {-qc }}\left(\mathcal{D}_{X}^{\bullet}, \mathcal{N}\right) \otimes_{\mathcal{O}_{X}} \mathcal{M}
$$

for any injective quasicoherent matrix factorization $\mathcal{N}$ and any quasicoherent matrix factorization $\mathcal{M}$ on $X$. As in Section B.2.3, $\mathcal{N}$ and $\mathcal{M}$ must be matrix factorizations of two sections $w^{\prime}$ and $w^{\prime \prime}$ of the same line bundle $\mathcal{L}$ on a scheme $X$; then the cotensor product $\mathcal{N} \square_{\mathcal{D}_{X}^{\bullet}} \mathcal{M}$ is a matrix factorization of the section $w^{\prime}+w^{\prime \prime}$ of the line bundle $\mathcal{L}$.

Remark B.2.6. While a matrix factorization version of Proposition B.2.5 is presented below, Remark B.2.3 explains the reason why a matrix factorization version of Theorem B.2.5 cannot be formulated in the way similar to the version for complexes of quasicoherent sheaves above. Still, let $\mathcal{N}$ and $\mathcal{M}$ be coherent matrix factorizations of sections $w^{\prime}$ and $w^{\prime \prime}$ of the same line bundle $\mathcal{L}$ on a separated Noetherian scheme $X$ with enough vector bundles. Let $\mathcal{P}$ and $\mathcal{Q}$ be coherent matrix factorizations of the potentials $-w^{\prime}$ and $-w^{\prime \prime} \in \mathcal{L}(X)$ isomorphic to $\mathcal{H}_{\text {om }}{ }_{X \text {-qc }}\left(\mathcal{N}, \mathcal{D}_{X}^{\bullet}\right)$ and $\mathcal{H} m_{X \text {-qc }}\left(\mathcal{M}, \mathcal{D}_{X}^{\bullet}\right)$ in the respected coderived categories.

Let $\mathcal{E}_{\bullet}$ and $\mathcal{F}_{\bullet}$ be left resolutions of the matrix factorizations $\mathcal{P}$ and $\mathcal{Q}$ by locally free matrix factorizations of finite rank (of the respected potentials). Then the totalizations of the bounded-below complexes of matrix factorizations $\mathcal{H}_{\text {om }}{ }_{X \mathrm{cc}}\left(\mathcal{E}_{\bullet}, \mathcal{D}_{X}^{\bullet}\right)$, $\mathcal{H o m}_{X \text {-qc }}\left(\mathcal{F}_{\bullet}, \mathcal{D}_{X}^{\bullet}\right)$, and $\mathcal{H o m}_{X \text {-qc }}\left(\mathcal{E}_{\bullet} \otimes_{\mathcal{O}_{X}} \mathcal{F}_{\bullet}, \mathcal{D}_{X}^{\bullet}\right)$ represent objects naturally isomorphic to $\mathcal{N}, \mathcal{M}$ and $\mathcal{N} \square_{\mathcal{D}_{X}^{\bullet}} \mathcal{M}$ in the coderived categories of matrix factorizations of the potentials $w^{\prime}, w^{\prime \prime}$, and $w^{\prime}+w^{\prime \prime}$ (cf. Corollary 2.5).

For any quasicoherent matrix factorizations $\mathcal{M}$ and $\mathcal{N}$ of sections $w^{\prime}$ and $w^{\prime \prime}$ of the same line bundle $\mathcal{L}$ on the scheme $X$, there is a natural morphism of quasicoherent matrix factorizations of the section $w^{\prime}+w^{\prime \prime}$ of the line bundle $\mathcal{L}$ on $X$

$$
\mathcal{N} \otimes_{\mathcal{O}_{X}} \mathcal{H o m}_{X \text {-qc }}\left(\mathcal{D}_{X}^{\bullet}, \mathcal{M}\right) \longrightarrow \mathcal{H o m}_{X \text {-qc }}\left(\mathcal{H o m}{ }_{X \text {-qc }}\left(\mathcal{N}, \mathcal{D}_{X}^{\bullet}\right), \mathcal{M}\right)
$$

constructed in the same way as it was done for complexes of quasicoherent sheaves in Section B.2.5. 
Proposition B.2.6. For any coherent matrix factorization $\mathcal{N}$ and injective quasicoherent matrix factorization $\mathcal{M}$ of sections $w^{\prime}$ and $w^{\prime \prime}$ of the same line bundle $\mathcal{L}$ on a separated Noetherian scheme $X$, the natural morphism (39) is an isomorphism in the coderived category of quasicoherent matrix factorizations of the potential $w^{\prime}+w^{\prime \prime} \in \mathcal{L}(X)$.

Proof. The argument follows the lines of the proof of Proposition B.2.5. The lefthand side of the desired isomorphism is well-defined as a functor of the argument $\mathcal{N} \in \mathrm{D}^{\mathrm{co}}\left(\left(X, \mathcal{L}, w^{\prime \prime}\right)\right.$-qcoh) taking values in $\mathrm{D}^{\mathrm{co}}\left(\left(X, \mathcal{L}, w^{\prime}+w^{\prime \prime}\right)\right.$-qcoh $)$, while the right-hand side is well-defined as a functor of the argument $\mathcal{N} \in \mathrm{D}^{\text {abs }}\left(\left(X, \mathcal{L}, w^{\prime \prime}\right)\right.$-coh $)$ taking values, say, in the same coderived category. Besides, the right-hand side, viewed as an object of the coderived category, only depends on the matrix factorization $\mathcal{H o m}_{X \text {-qc }}\left(\mathcal{N}, \mathcal{D}_{X}^{\bullet}\right)$ viewed as an object of the absolute derived category.

Furthermore, the contravariant Serre-Grothendieck duality $\mathcal{H o m}_{X \text {-qc }}\left(-, \mathcal{D}_{X}^{\bullet}\right)$ is well-defined as a functor $\mathrm{D}^{\text {abs }}\left(\left(X, \mathcal{L}, w^{\prime \prime}\right)\right.$-qcoh $) \rightarrow \mathrm{D}^{\text {abs }}\left(\left(X, \mathcal{L},-w^{\prime \prime}\right)\right.$-qcoh $)$ and takes $\mathrm{D}^{\text {abs }}\left(\left(X, \mathcal{L}, w^{\prime \prime}\right)\right.$-coh $) \subset \mathrm{D}^{\text {abs }}\left(\left(X, \mathcal{L}, w^{\prime \prime}\right)\right.$-qcoh $)$ into $\mathrm{D}^{\text {abs }}\left(\left(X, \mathcal{L},-w^{\prime \prime}\right)\right.$-coh $) \subset$ $\mathrm{D}^{\text {abs }}\left(\left(X, \mathcal{L},-w^{\prime \prime}\right)\right.$-coh $)$, inducing an equivalence between these two subcategories (see Proposition 2.5). In particular, one can conclude that all the functors involved are local in $X$, and it suffices to prove the desired assertion for matrix factorizations over affine open subschemes $U \subset X$.

Now let $\mathcal{K}$ be a coherent matrix factorization of the potential $-w^{\prime \prime}$ isomorphic to $\mathcal{H} m_{U \text {-qc }}\left(\mathcal{N}, \mathcal{D}_{U}^{\bullet}\right)$ in $\mathrm{D}^{\text {abs }}\left(\left(U, \mathcal{L},-w^{\prime \prime}\right)\right.$-qcoh), and let $\mathcal{E}_{\bullet}$ be its left resolution by locally free matrix factorizations of the same potential $-w^{\prime \prime} \in \mathcal{L}(U)$. Then the matrix factorization $\operatorname{Hom}_{X \text {-qc }}(\mathcal{K}, \mathcal{M})$ is isomorphic in $\mathrm{D}^{\mathrm{co}}\left(\left(X, \mathcal{L}, w^{\prime}+w^{\prime \prime}\right)\right.$-qcoh) to the totalization of the complex of matrix factorizations $\mathcal{H o m}_{X \text {-qc }}\left(\mathcal{E}_{\bullet}, \mathcal{M}\right)$ constructed by taking infinite direct sums along the diagonals; and the matrix factorization $\mathcal{N} \simeq \mathcal{H o m}_{X \text {-qc }}\left(\mathcal{K}, \mathcal{D}_{X}^{\bullet}\right)$ can be described similarly (cf. the proof of Corollary 2.5).

It remains to notice that the functor of tensoring with $\mathcal{H o m}_{X \text {-qc }}\left(\mathcal{E}_{\bullet}, \mathcal{O}_{X}\right)$ and totalizing by taking infinite direct sums along the diagonals takes the homotopy equivalence

$$
\mathcal{D}_{X}^{\bullet} \otimes_{\mathcal{O}_{X}} \mathcal{H o m}_{X \text {-qc }}\left(\mathcal{D}_{X}^{\bullet}, \mathcal{M}\right) \longrightarrow \mathcal{M}
$$

to a homotopy equivalence of matrix factorizations.

As in Section B.2.5, we finish by discussing the case of a separated scheme $X$ of finite type over a field $k$. From now on we also assume that $\mathcal{L}=\mathcal{O}_{X}$. So let $w^{\prime}, w^{\prime \prime} \in \mathcal{O}(X)$ be two global regular functions on $X$; as in Section B.2.2, we consider the regular function $w_{1}^{\prime}+w_{2}^{\prime \prime}=p_{1}^{*} w^{\prime}+p_{2}^{*} w^{\prime \prime}$ on $X \times_{k} X$. We use the dualizing complexes $\mathcal{D}_{X}^{\bullet}=\pi^{+} \mathcal{O}_{\text {Spec } k}$ and $\mathcal{D}_{X \times_{k} X}^{\bullet}=\left(\pi \times{ }_{k} \pi\right)^{+} \mathcal{O}_{\text {Spec } k}$.

The equivalence of categories

$$
\mathrm{D}^{\mathrm{co}}\left(\left(X \times_{k} X, \mathcal{O}, w_{1}^{\prime}+w_{2}^{\prime \prime}\right)-\mathrm{qcoh} \mathrm{fl}_{\mathrm{fl}}\right) \simeq \mathrm{D}^{\mathrm{co}}\left(\left(X \times_{k} X, \mathcal{O}, w_{1}^{\prime}+w_{2}^{\prime \prime}\right)-\mathrm{qcoh}\right)
$$


constructed using the dualizing complex $\mathcal{D}_{X \times_{k} X}^{\bullet}$ and the similar equivalences of coderived categories of matrix factorizations of the potentials $w^{\prime}$ and $w^{\prime \prime}$ on $X$ constructed using the dualizing complex $\mathcal{D}_{X}^{\bullet}$ transform the external tensor product functor (cf. (9))

$\otimes_{k}: \mathrm{D}^{\mathrm{co}}\left(\left(X, \mathcal{O}, w^{\prime}\right)-\mathrm{q} \operatorname{coh}_{\mathrm{fl}}\right) \times \mathrm{D}^{\mathrm{co}}\left(\left(X, \mathcal{O}, w^{\prime \prime}\right)-\mathrm{qcoh}_{\mathrm{fl}}\right)$

$\longrightarrow \mathrm{D}^{\mathrm{co}}\left(\left(X \times_{k} X, \mathcal{O}, w_{1}^{\prime}+w_{2}^{\prime \prime}\right)-\mathrm{q} \operatorname{coh}_{\mathrm{fl}}\right)$

into the external tensor product functor

$\otimes_{k}: \mathrm{D}^{\mathrm{co}}\left(\left(X, \mathcal{O}, w^{\prime}\right)-\mathrm{qcoh}\right) \times \mathrm{D}^{\mathrm{co}}\left(\left(X, \mathcal{O}, w^{\prime \prime}\right)-\mathrm{qcoh}\right)$

$$
\longrightarrow \mathrm{D}^{\mathrm{co}}\left(\left(X \times_{k} X, \mathcal{O}, w_{1}^{\prime}+w_{2}^{\prime \prime}\right)-\mathrm{qcoh}\right)
$$

since so do the functors $\mathcal{D}_{X}^{\bullet} \otimes_{\mathcal{O}_{X}}-$ and $\mathcal{D}_{X \times_{k} X}^{\bullet} \otimes_{\mathcal{O}_{X \times{ }_{k} X}}-$.

Let $\mathcal{N}$ and $\mathcal{M}$ be injective quasicoherent matrix factorizations of the potentials $w^{\prime}$ and $w^{\prime \prime}$ on $X$, and let $\mathcal{J}$ be an injective quasicoherent matrix factorization of the potential $w_{1}^{\prime}+w_{2}^{\prime \prime}$ on $X \times_{k} X$ isomorphic to $\mathcal{N} \otimes_{k} \mathcal{M}$ in

$$
\mathrm{D}^{\mathrm{co}}\left(\left(X \times_{k} X, \mathcal{O}, w_{1}^{\prime}+w_{2}^{\prime \prime}\right)-\mathrm{qcoh}\right) .
$$

Then in the coderived categories of quasicoherent matrix factorizations one has

$$
\begin{aligned}
\mathcal{N} \square_{\mathcal{D}_{X}^{\bullet}} \mathcal{M}=\mathcal{D}_{X}^{\bullet} \otimes_{\mathcal{O}_{X}} \Delta^{*} & \left(\mathcal{H o m}_{\mathcal{O}_{X}}\left(\mathcal{D}_{X}^{\bullet}, \mathcal{N}\right) \otimes_{k} \mathcal{H o m}_{\mathcal{O}_{X}}\left(\mathcal{D}_{X}^{\bullet}, \mathcal{M}\right)\right) \\
& \simeq \mathcal{D}_{X}^{\bullet} \otimes_{\mathcal{O}_{X}} \Delta^{*} \mathcal{H o m}_{\mathcal{O}_{X}}\left(\mathcal{D}_{X \times_{k} X}^{\bullet}, \mathcal{J}\right) \simeq \mathbb{R} \Delta^{!}\left(\mathcal{N} \otimes_{k} \mathcal{M}\right)
\end{aligned}
$$

by the result of Theorem 3.8 applied to the proper morphism $\Delta$. We have obtained the formula

$$
\mathcal{N} \square_{\pi}+\mathcal{O}_{\text {Spec } k} \mathcal{M} \simeq \mathbb{R} \Delta^{!}\left(\mathcal{N} \otimes_{k} \mathcal{M}\right)
$$

for the cotensor product of quasicoherent matrix factorizations on the scheme $X$.

B.2.7. Hochschild homology. Let $X$ be a separated scheme of finite type over a field $k$ and $\pi: X \rightarrow$ Spec $k$ be its structure morphism. Let $w \in \mathcal{O}(X)$ be a global regular function; as in Section B.2.4, we assume that the morphism of schemes $w: X \rightarrow \mathbb{A}_{k}^{1}$ is flat. Consider the scheme $X \times_{k} X$ and endow it with the potential $w_{2}-w_{1}=p_{2}^{*}(w)-p_{1}^{*}(w)$. Let $\Delta: X \rightarrow X \times_{k} X$ denote the diagonal morphism.

Theorem B.2.7. In the assumptions of Theorem B.2.4, there is a natural isomorphism between the Hochschild homology module $H_{*}\left(\mathrm{DG}^{\text {abs }}((X, \mathcal{O}, w)\right.$-coh $\left.)\right)$ over the algebra $H H^{*}\left(\mathrm{DG}^{\mathrm{abs}}((X, \mathcal{O}, w)\right.$-coh $\left.)\right)$ and the Ext module

$$
\operatorname{Hom}_{D^{c o}\left(\left(X \times_{k} X, \mathcal{O}, w_{2}-w_{1}\right)-q c o h\right)}\left(\Delta_{*} \mathcal{O}_{X}, \Delta_{*} \mathcal{D}_{X}^{\bullet}[*]\right)
$$


over the algebra

$$
\operatorname{Hom}_{\mathrm{D}^{\mathrm{co}}\left(\left(X \times_{k} X, \mathcal{O}, w_{2}-w_{1}\right) \text {-qcoh }\right)}\left(\Delta_{*} \mathcal{D}_{X}^{\bullet}, \Delta_{*} \mathcal{D}_{X}^{\bullet}[*]\right) .
$$

Here $\mathcal{D}_{X}^{\bullet}$ denotes the dualizing complex $\pi^{+} \mathcal{O}_{\text {Spec } k}$ on $X$.

Proof. By the definition, the Hochschild homology of a $\mathbb{Z} / 2$-graded DG-category $D G$ is the $\mathbb{Z} / 2$-graded vector space $\operatorname{Tor}_{*}^{\mathrm{DG} \otimes_{k} \mathrm{DG} \text { (D) }}$ (DG, DG) for the diagonal right and left DG-modules DG over the DG-category DG $\otimes_{k}$ DG ${ }^{\circ}$ [Polishchuk and Positselski 2012, Sections 2.4 and 3.1]. This is the conventional derived tensor product ("of the first kind") of a left and a right DG-module over a small DG-category. The Hochschild cohomology algebra $\operatorname{Hom}_{\mathrm{D}\left(\mathrm{DG} \otimes_{k} \mathrm{DG}\right.}{ }^{\text {op }}(\mathrm{DG}, \mathrm{DG}[*])$ acts on the Hochschild homology space via its action on, say, the first argument of the Tor.

As in the proof of Theorem B.2.4, we set $\mathrm{DG}_{w}=\mathrm{DG}^{\text {abs }}((X, \mathcal{O}, w)$-coh); accordingly, $\mathrm{DG}_{-w}=\mathrm{DG}^{\mathrm{abs}}((X, \mathcal{O},-w)$-coh $)$ and

$$
\mathrm{DG}_{w_{2}-w_{1}}=\mathrm{DG}^{\mathrm{abs}}\left(\left(X \times_{k} X, \mathcal{O}, w_{2}-w_{1}\right)-\mathrm{coh}\right) .
$$

The DG-functor $\mathrm{DG}_{w}^{\mathrm{op}} \otimes_{k} \mathrm{DG}_{w} \rightarrow \mathrm{DG}_{w_{2}-w_{1}}$ (29) induces a fully faithful functor between the homotopy categories $H^{0}\left(\mathrm{DG}_{w}\right)^{\mathrm{op}} \otimes_{k} H^{0}\left(\mathrm{DG}_{w}\right) \rightarrow H^{0}\left(\mathrm{DG}_{w_{2}-w_{1}}\right)$ such that every object in the target category can be obtained from objects in the image using the operations of a cone and the passage to a direct summand.

Let $\mathrm{DG}\left(\bmod -\mathrm{DG}_{w}^{\mathrm{op}} \otimes_{k} \mathrm{DG}_{w}\right)$ denote the DG-category version of the (conventional) derived category of right DG-modules over the DG-category $\mathrm{DG}_{w}^{\text {op }} \otimes_{k} \mathrm{DG}$ (i.e., contravariant DG-functors from $D G_{w}^{o p} \otimes_{k} D G$ into the DG-category $D G(k$-vect) of $\mathbb{Z} / 2$-graded complexes of $k$-vector spaces $)$. Let $D G\left(\bmod -D G_{w}^{o p} \otimes_{k} D_{w}\right)^{0} \subset$ $\mathrm{DG}\left(\bmod -\mathrm{DG}_{w}^{\mathrm{op}} \otimes_{k} \mathrm{DG}_{w}\right)$ denote the full DG-subcategory of DG-modules corresponding to compact objects of the derived category $D\left(\bmod -D G_{w}^{o p} \otimes_{k} D G_{w}\right)$ of right DG-modules.

The derived tensor product with the left DG-module $\mathrm{DG}_{w}$ over $\mathrm{DG}_{w}^{\mathrm{op}} \otimes_{k} \mathrm{DG}_{w}$ can be viewed as a covariant DG-functor $\mathrm{DG}\left(\bmod -\mathrm{DG}_{w}^{\mathrm{op}} \otimes_{k} \mathrm{DG}_{w}\right) \rightarrow \mathrm{DG}(k$-vect $)$. We are interested in the restriction of this DG-functor to the DG-subcategory $\mathrm{DG}\left(\bmod -\mathrm{DG}_{w}^{\mathrm{op}} \otimes_{k} \mathrm{DG}_{w}\right)^{0}$; let us denote it by

$$
F: \mathrm{DG}\left(\bmod -\mathrm{DG}_{w}^{\mathrm{op}} \otimes_{k} \mathrm{DG}_{w}\right)^{0} \longrightarrow \mathrm{D}(k \text {-vect }) .
$$

There is a natural DG-functor $\mathrm{DG}_{w}^{\mathrm{op}} \otimes_{k} \mathrm{DG}_{w} \rightarrow \mathrm{DG}\left(\bmod -\mathrm{DG}_{w}^{\mathrm{op}} \otimes_{k} \mathrm{DG}_{w}\right)^{0}$ assigning to any object of $D G_{w}^{o p} \otimes_{k} D G_{w}$ the contravariant DG-functor represented by it. Similarly one constructs a DG-functor $\mathrm{DG}_{w_{2}-w_{1}} \rightarrow \mathrm{DG}\left(\bmod -\mathrm{DG}_{w}^{\mathrm{op}} \otimes_{k} \mathrm{DG}_{w}\right)^{0}$ whose composition with the DG-functor $D G_{w}^{o p} \otimes_{k} D G_{w} \rightarrow D G_{w_{2}-w_{1}}$ is naturally quasi-isomorphic to the DG-functor $\mathrm{DG}_{w}^{\mathrm{op}} \otimes_{k} \mathrm{DG}_{w} \rightarrow \mathrm{DG}\left(\bmod -\mathrm{DG}_{w}^{\mathrm{op}} \otimes_{k} \mathrm{DG}_{w}\right)^{0}$.

It is claimed that the composition of the DG-functor

$$
\mathrm{DG}_{w_{2}-w_{1}} \longrightarrow \mathrm{DG}\left(\bmod -\mathrm{DG}_{w}^{\mathrm{op}} \otimes_{k} \mathrm{DG}_{w}\right)^{0}
$$


with the DG-functor $F: \mathrm{DG}\left(\bmod -\mathrm{DG}_{w}^{\mathrm{op}} \otimes_{k} \mathrm{DG}_{w}\right)^{0} \rightarrow \mathrm{D}(k$-vect $)$ is naturally quasiisomorphic to the DG-functor $\operatorname{Hom}_{\mathrm{DG}_{w_{2}-w_{1}}}\left(\Delta_{*} \mathcal{O}_{X},-\right)$. Since the derived categories of left DG-modules over $\mathrm{DG}_{w_{1}-w_{2}}$ and $\mathrm{DG} \mathrm{G}_{w}^{\mathrm{op}} \otimes_{k} \mathrm{DG} w$ are equivalent, it suffices to construct a quasi-isomorphism between the compositions of the two DG-functors in question with the DG-functor $\mathrm{DG}_{w}^{\mathrm{op}} \otimes_{k} \mathrm{DG}_{w} \rightarrow \mathrm{DG}_{w_{2}-w_{1}}$.

Indeed, let $\left(\mathcal{K}^{\mathrm{op}}, \mathcal{M}\right)$ be an object of $D G_{w}^{\text {op }} \otimes_{k} D G_{w}$. Then the functor of the (derived or underived) tensor product with the diagonal left DG-module $D_{w}$ takes the right DG-module over $D G_{w}^{o p} \otimes_{k} D G_{w}$ represented by $\left(\mathcal{K}^{\mathrm{op}}, \mathcal{M}\right)$ to the complex of $k$-vector spaces $\operatorname{Hom}_{\mathrm{DG}_{w}}(\mathcal{K}, \mathcal{M})$. Substituting $\mathcal{K}=\mathcal{H o m}_{X \text {-qc }}\left(\mathcal{N}, \mathcal{D}_{X}^{\bullet}\right)$ with $\mathcal{N} \in \mathrm{DG}_{-w}$ and assuming $\mathcal{M}$ to be represented by an injective matrix factorization isomorphic to the given coherent one in $\mathrm{DG}^{\mathrm{co}}((X, \mathcal{O}, w)$-qcoh), we have to compute the complex of $k$-vector spaces $\operatorname{Hom}_{(X, \mathcal{O}, w) \text {-qcoh }}\left(\mathcal{H o m}_{X \text {-qc }}\left(\mathcal{N}, \mathcal{D}_{X}^{\bullet}\right), \mathcal{M}\right)$.

Now the formula (24) together with Lemma B.2.3 allow us to interpret this complex as $\mathbb{R} \Gamma\left(X, \mathcal{H o m}_{X \text {-qc }}\left(\mathcal{H}_{\text {om }}\right.\right.$-qc $\left.\left._{\text {-q }}\left(\mathcal{N}, \mathcal{D}_{X}^{\bullet}\right), \mathcal{M}\right)\right)$. According to Proposition B.2.6 together with the formula (40), this is the same as $\mathbb{R} \Gamma\left(X, \mathbb{R} \Delta^{!}\left(\mathcal{N} \otimes_{k} \mathcal{M}\right)\right)$, or, in other notation, $\operatorname{Hom}_{\mathrm{DG}}{ }^{\mathrm{co}}((X, \mathcal{O}, 0)$-qcoh $)\left(\mathcal{O}_{X}, \mathbb{R} \Delta^{!}\left(\mathcal{N} \otimes_{k} \mathcal{M}\right)\right)$. Finally, the adjunction of $\Delta_{*}$ and $\mathbb{R} \Delta^{!}$allows us to rewrite the complex in question as

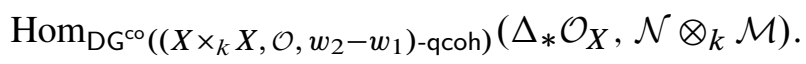

The desired quasi-isomorphism of DG-functors is obtained.

It remains to recall that, according to the proof of Theorem B.2.4, the diagonal right DG-module $\mathrm{DG}_{w}$ over $\mathrm{DG}_{w}^{\mathrm{op}} \otimes_{k} \mathrm{DG}_{w}$ is represented by the object $\Delta_{*} \mathcal{D}_{X}^{\bullet} \in$ $\mathrm{DG}_{w_{2}-w_{1}}$, in order to finish our proof here.

Remark B.2.7. The Hochschild homology module $H H_{*}\left(\left(\mathrm{DG}^{\mathrm{abs}}(X, \mathcal{O}, w)\right.\right.$-coh $\left.)\right)$ over the Hochschild cohomology algebra $H H^{*}\left(\left(\mathrm{DG}^{\text {abs }}(X, \mathcal{O}, w)\right.\right.$-coh $\left.)\right)$ can be also computed as the Ext module

$$
\operatorname{Hom}_{D^{c o}\left(\left(X \times_{k} X, \mathcal{O}, w_{1}-w_{2}\right)-q c o h\right)}\left(\Delta_{*} \mathcal{O}_{X}, \Delta_{*} \mathcal{D}_{X}^{\bullet}[*]\right)
$$

over the Ext algebra

$$
\operatorname{Hom}_{\mathrm{D}^{\mathrm{abs}}\left(\left(X \times_{k} X, \mathcal{O}, w_{1}-w_{2}\right)-\mathrm{coh}\right)}\left(\Delta_{*} \mathcal{O}_{X}, \Delta_{*} \mathcal{O}_{X}[*]\right)^{\mathrm{op}},
$$

according to Remark B.2.4. Moreover, the contravariant Serre duality for matrix factorizations over $X \times_{k} X$ can be used in order to obtain an alternative proof of our Hochschild homology computation. Indeed, for any coherent matrix factorizations 
$\mathcal{N}$ and $\mathcal{M}$ of the potentials $-w$ and $w$ on $X$ there are natural quasi-isomorphisms

$$
\begin{aligned}
& \operatorname{Hom}_{\mathrm{DG}^{\text {abs }}((X, \mathcal{O}, w) \text {-coh })}\left(\mathcal{H o m}_{X \text {-qc }}\left(\mathcal{N}, \mathcal{D}_{X}^{\bullet}\right), \mathcal{M}\right) \\
& \simeq \operatorname{Hom}_{\mathrm{DG}^{\mathrm{abs}}((X, \mathcal{O}, w) \text {-coh) }}\left(\mathcal{H o m}_{X \text {-qc }}\left(\mathcal{N}, \mathcal{D}_{X}^{\bullet}\right), \mathcal{H} \mathrm{m}_{X \text {-qc }}\left(\mathcal{H o m}_{X \text {-qc }}\left(\mathcal{M}, \mathcal{D}_{X}^{\bullet}\right), \mathcal{D}_{X}^{\bullet}\right)\right) \\
& \simeq \operatorname{Hom}_{\mathrm{DG}^{\mathrm{abs}}\left(\left(X \times_{k} X, \mathcal{O}, w_{1}-w_{2}\right)\right. \text {-coh) }}\left(\mathcal{H o m}_{X \text {-qc }}\left(\mathcal{N}, \mathcal{D}_{X}^{\bullet}\right) \otimes_{k} \mathcal{H} \operatorname{Hom}_{X \text {-qc }}\left(\mathcal{M}, \mathcal{D}_{X}^{\bullet}\right), \Delta_{*} \mathcal{D}_{X}^{\bullet}\right) \\
& \simeq \operatorname{Hom}_{\mathrm{DG}^{\mathrm{abs}}\left(\left(X \times_{k} X, \mathcal{O}, w_{1}-w_{2}\right) \text {-coh }\right)}\left(\mathcal{H} o m_{X \times{ }_{k} X \text {-qc }}\left(\mathcal{N} \otimes_{k} \mathcal{M}, \mathcal{D}_{X \times_{k} X}^{\bullet}\right), \Delta_{*} \mathcal{D}_{X}^{\bullet}\right) \\
& \simeq \operatorname{Hom}_{\mathrm{DG}^{\mathrm{abs}}\left(\left(X \times_{k} X, \mathcal{O}, w_{2}-w_{1}\right) \text {-coh }\right)}\left(\Delta_{*} \mathcal{O}_{X}, \mathcal{N} \otimes_{k} \mathcal{M}\right)
\end{aligned}
$$

by Proposition 2.5 and the proof of Theorem B.2.4. In other words, while the right diagonal DG-module $\mathrm{DG}_{w}$ over $\mathrm{DG}_{w}^{\mathrm{op}} \otimes_{k} \mathrm{DG}_{w}$ is represented by the object

$$
\Delta_{*} \mathcal{D}_{X}^{\bullet} \in \mathrm{DG}^{\mathrm{abs}}\left(\left(X \times_{k} X, \mathcal{O}, w_{2}-w_{1}\right)-\mathrm{coh}\right)
$$

as a contravariant DG-functor on $\mathrm{DG}_{w}^{\mathrm{op}} \otimes_{k} \mathrm{DG}_{w} \subset \mathrm{DG}_{w_{2}-w_{1}}$, the left diagonal DG-bimodule $\mathrm{DG}_{w}$ over $\mathrm{DG}_{w}^{\mathrm{op}} \otimes_{k} \mathrm{DG}_{w}$ is represented by the object

$$
\Delta_{*} \mathcal{O}_{X}^{\bullet} \in \mathrm{DG}^{\mathrm{abs}}\left(\left(X \times_{k} X, \mathcal{O}, w_{2}-w_{1}\right)-\mathrm{coh}\right)
$$

as a covariant DG-functor on

$$
\mathrm{DG}_{w}^{\mathrm{op}} \otimes_{k} \mathrm{DG}_{w} \subset \mathrm{DG}_{w_{2}-w_{1}}=\mathrm{DG}^{\text {abs }}\left(\left(X \times_{k} X, \mathcal{O}, w_{2}-w_{1}\right) \text {-coh }\right) .
$$

B.2.8. Direct sum over the critical values. Let $X$ be a separated scheme of finite type over a field $k$ and $\pi: X \rightarrow$ Spec $k$ be its structure morphism. As in Sections B.2.5-B.2.7 (see also Section 3.7), we choose the dualizing complex $\mathcal{D}_{X}^{\bullet} \simeq \pi^{+} \mathcal{O}_{\text {Spec } k}$ on $X$. Let $w \in \mathcal{O}(X)$ be a global regular function on $X$ such that the morphism of schemes $w: X \rightarrow \mathbb{A}_{k}^{1}$ is flat (cf. [Orlov 2004; 2012]).

Let $c_{1}, \ldots, c_{n} \in k$ be a finite number of different elements of the ground field. Assume that there exist closed subschemes $Z_{i} \subset X$ such that the function $w$ is noncritical on $X \backslash\left(Z_{1} \cup \cdots \cup Z_{n}\right)$, the restriction of $w$ to $Z_{i}$ is equal to the constant $c_{i}$, and the schemes $Z_{i}$ admit smooth stratifications over $k$.

In particular, if the field $k$ is perfect, it suffices to require that the function $w$ has only a finite number of critical values $c_{1}, \ldots, c_{n} \in \mathbb{A}_{k}^{1}$ (i.e., the open subscheme $\mathbb{A}_{k, f}^{1} \subset \mathbb{A}_{k}^{1}$ is nonempty; see Section B.2.1), and all of these values belong to the field $k$ (rather than its algebraic closure). When the field $k$ has zero characteristic, the former condition holds automatically. Then one simply takes $Z_{i}$ to be the zero locus of the function $w_{i}-c_{i}$ on $X$.

Consider the Cartesian square $X \times_{k} X$ with the global function $w_{2}-w_{1}=$ $p_{2}^{*}(w)-p_{1}^{*}(w)$ on it. Let $\Delta: X \rightarrow X \times_{k} X$ denote the diagonal morphism. The following result is to be compared with [Polishchuk and Positselski 2012, Corollary 4.10]. 
Corollary B.2.8. There are natural isomorphisms of $\mathbb{Z} / 2$-graded $k$-algebras

$$
\begin{aligned}
\bigoplus_{i=1}^{n} H H^{*}\left(\mathrm{DG}^{\mathrm{abs}}\right. & \left.\left(\left(X, \mathcal{O}, w-c_{i}\right)-\mathrm{coh}\right)\right) \\
& \simeq \operatorname{Hom}_{\mathrm{D}^{\mathrm{co}}\left(\left(X \times_{k} X, \mathcal{O}, w_{2}-w_{1}\right) \text {-qcoh }\right)}\left(\Delta_{*} \mathcal{D}_{X}^{\bullet}, \Delta_{*} \mathcal{D}_{X}^{\bullet}[*]\right) \\
& \simeq \operatorname{Hom}_{\mathrm{D}^{\mathrm{abs}}\left(\left(X \times_{k} X, \mathcal{O}, w_{1}-w_{2}\right)-\mathrm{coh}\right)}\left(\Delta_{*} \mathcal{O}_{X}, \Delta_{*} \mathcal{O}_{X}[*]\right)^{\mathrm{op}} .
\end{aligned}
$$

There are also natural isomorphisms of $\mathbb{Z} / 2$-graded $k$-modules

$$
\begin{aligned}
& \bigoplus_{i=1}^{n} H H_{*}\left(\mathrm{DG}^{\mathrm{abs}}\left(\left(X, \mathcal{O}, w-c_{i}\right)-\mathrm{coh}\right)\right) \\
& \simeq \operatorname{Hom}_{\mathrm{D}^{\mathrm{co}}\left(\left(X \times_{k} X, \mathcal{O}, w_{2}-w_{1}\right) \text {-qcoh }\right)}\left(\Delta_{*} \mathcal{O}_{X}, \Delta_{*} \mathcal{D}_{X}^{\bullet}[*]\right) \\
& \simeq \operatorname{Hom}_{\mathrm{D}^{\mathrm{co}}\left(\left(X \times_{k} X, \mathcal{O}, w_{1}-w_{2}\right) \text {-qcoh }\right)}\left(\Delta_{*} \mathcal{O}_{X}, \Delta_{*} \mathcal{D}_{X}^{\bullet}[*]\right)
\end{aligned}
$$

over the $\mathbb{Z} / 2$-graded $k$-algebra (41).

Proof. For each $i=1, \ldots, n$, let $Y_{i}$ denote the open subscheme $X \backslash \bigcup_{j \neq i} Z_{i} \subset X$. Let $w_{i} \in \mathcal{O}\left(Y_{i}\right)$ denote the restriction of the regular function $w-c_{i}$ to $Y_{i}$. The argument is based on the results of Sections B.2.4 and B.2.7 applied to the schemes $Y_{i}$ (or their open subschemes) endowed with the potentials $w_{i}$.

The restriction of morphisms (in the coderived categories) of quasicoherent matrix factorizations to the open subschemes $Y_{i} \subset X$ defines a $\mathbb{Z} / 2$-graded $k$-algebra morphism from the (middle or) right-hand side to the left-hand side of (41), and a $\mathbb{Z} / 2$-graded $k$-module morphism from the (middle or) right-hand side to the left-hand side of (42). It remains to show that these morphisms are isomorphisms.

For this purpose, one can start with replacing $\Delta_{*} \mathcal{D}_{X}^{\bullet}$ or $\Delta_{*} \mathcal{O}_{X}$ in the second argument of the Hom spaces in the middle or right-hand sides of (41) and (42) with an injective matrix factorization $\mathcal{J}$ on $X \times_{k} X$ representing the same object in the coderived category. Then one notices that the restriction from $X \times_{k} X$ to its open subscheme $V=\bigcup_{i=1}^{n} Y_{i} \times_{k} Y_{i}$ does not change the Hom spaces in the right-hand sides, as the image of $\Delta$ is contained in $V$.

Finally, one writes down the Čech resolution of the matrix factorization $\left.\mathcal{J}\right|_{V}$ corresponding to the covering of the scheme $V$ by its open subschemes $Y_{i} \times_{k} Y_{i}$. This is a finite acyclic complex of injective matrix factorizations, so applying the functor $\operatorname{Hom}_{\left(V, \mathcal{O},\left.\left(w_{2}-w_{1}\right)\right|_{V}\right) \text {-qcoh }}(\mathcal{K},-)$ from any quasicoherent matrix factorization $\mathcal{K}$ preserves its acyclicity. Since the Hom spaces on any intersection of at least two different open subschemes in the covering are zero by Theorems B.2.4- B.2.7 (as $w$ is noncritical on $Y_{i} \cap Y_{j}$ for any $i \neq j$ ), the desired isomorphisms follow.

Remark B.2.8. The Hochschild cohomology algebra and the Hochschild homology module of the DG-category version $\mathrm{DG}^{\mathrm{b}}(X$-coh) of the bounded derived category $\mathrm{D}^{\mathrm{b}}(X$-coh) of (complexes of) coherent sheaves on a separated scheme $X$ of finite type over a field $k$ can be computed in the way similar to (but simpler than) the 
above. The answers are the same as in Theorems B.2.4 and B.2.7:

$$
\begin{aligned}
H H^{*}\left(\mathrm{DG}^{\mathrm{b}}(X-\mathrm{coh})\right) \simeq \operatorname{Hom}_{\mathrm{D}(} & \left.X \times_{k} X \text {-qcoh }\right)\left(\Delta_{*} \mathcal{D}_{X}^{\bullet}, \Delta_{*} \mathcal{D}_{X}^{\bullet}[*]\right) \\
& \simeq \operatorname{Hom}_{\mathrm{D}^{\mathrm{b}}\left(X \times_{k} X \text {-coh }\right)}\left(\Delta_{*} \mathcal{O}_{X}, \Delta_{*} \mathcal{O}_{X}[*]\right)^{\mathrm{op}}
\end{aligned}
$$

and

$$
H H_{*}\left(\mathrm{DG}^{\mathrm{b}}(X \text {-coh })\right) \simeq \operatorname{Hom}_{\mathrm{D}\left(X \times_{k} X \text {-qcoh }\right)}\left(\Delta_{*} \mathcal{O}_{X}, \Delta_{*} \mathcal{D}_{X}^{\bullet}[*]\right),
$$

the only difference being that $\mathrm{DG}^{\mathrm{b}}(X$-coh) is a $\mathbb{Z}$-graded DG-category and the righthand sides describe the Hochschild (co)homology as a $\mathbb{Z}$-graded algebra and module. The only assumption is that the scheme $X$ should admit a smooth stratification over $k$ (i.e., it suffices that the field $k$ be perfect).

\section{Acknowledgments}

Positselski is grateful to Daniel Pomerleano and Kevin Lin for sending him a preliminary version of their manuscript [Lin and Pomerleano 2013], which inspired the present work. We also wish to thank Roman Bezrukavnikov, Alexander Kuznetsov, and Amnon Neeman for helpful conversations, Alexander Polishchuk and Henning Krause for stimulating questions, and Dmitri Orlov for his insightful remarks on an early version of this manuscript. Finally, Positselski would like to thank an anonymous referee of an early version for detailed suggestions on the improvement of the exposition. It was initially my attempt to implement those suggestions that eventually resulted in the growth of the size of this paper by a factor of almost seven. Efimov was partially supported by RFBR grant 2998.2014.1 and RFBR research project 15-51-50045. Positselski was supported in part by a Simons Foundation grant and RFBR grants in Moscow and by a fellowship from the Lady Davis Foundation at the Technion. The paper was prepared within the framework of a subsidy granted to the HSE by the Government of the Russian Federation for the implementation of the Global Competitiveness Program.

\section{References}

[Balmer and Schlichting 2001] P. Balmer and M. Schlichting, "Idempotent completion of triangulated categories”, J. Algebra 236:2 (2001), 819-834. MR 2002a:18013 Zbl 0977.18009

[Bass 1963] H. Bass, "Big projective modules are free", Illinois J. Math. 7 (1963), 24-31. MR 26 \#1341 Zbl 0115.26003

[Becker 2014] H. Becker, "Models for singularity categories", Adv. Math. 254 (2014), 187-232. MR 3161097 Zbl 06284998

[Bourbaki 1980] N. Bourbaki, Algèbre homologique: Chapitre 10, Masson, Paris, 1980. MR 82j:18022 Zbl 0455.18010

[Buchweitz 1986] R.-O. Buchweitz, "Maximal Cohen-Macaulay modules and Tate-cohomology over Gorenstein rings", preprint, 1986, available at http://hdl.handle.net/1807/16682. 
[Cartan and Eilenberg 1956] H. Cartan and S. Eilenberg, Homological algebra, Princeton University Press, 1956. MR 17,1040e Zbl 0075.24305

[Chen 2010] X.-W. Chen, "Unifying two results of Orlov on singularity categories", Abh. Math. Semin. Univ. Hambg. 80:2 (2010), 207-212. MR 2011j:14036 Zbl 1214.18013

[Drinfeld 2004] V. Drinfeld, "DG quotients of DG categories", J. Algebra 272:2 (2004), 643-691. MR 2006e:18018 Zbl 1064.18009

[Drinfeld 2006] V. Drinfeld, "Infinite-dimensional vector bundles in algebraic geometry: an introduction", pp. 263-304 in The unity of mathematics, edited by P. Etingof et al., Progr. Math. 244, Birkhäuser, Boston, 2006. MR 2007d:14038 Zbl 1108.14012

[Dyckerhoff and Murfet 2013] T. Dyckerhoff and D. Murfet, "Pushing forward matrix factorizations", Duke Math. J. 162:7 (2013), 1249-1311. MR 3079249 Zbl 1273.14014

[Efimov 2012] A. I. Efimov, "Cyclic homology of categories of matrix factorizations", preprint, 2012. arXiv 1212.2859

[Efimov 2013] A. I. Efimov, "Homotopy finiteness of some DG categories from algebraic geometry", preprint, 2013. arXiv 1308.0135

[Eisenbud 1980] D. Eisenbud, "Homological algebra on a complete intersection, with an application to group representations", Trans. Amer. Math. Soc. 260:1 (1980), 35-64. MR 82d:13013 Zbl 0444.13006

[Getzler and Jones 1990] E. Getzler and J. D. S. Jones, " $A_{\infty}$-algebras and the cyclic bar complex", Illinois J. Math. 34:2 (1990), 256-283. MR 91e:19001 Zbl 0701.55009

[Goodearl and Warfield 1989] K. R. Goodearl and R. B. Warfield, Jr., An introduction to noncommutative Noetherian rings, London Mathematical Society Student Texts 16, Cambridge University Press, 1989. MR 91c:16001 Zbl 0679.16001

[Grothendieck 1961] A. Grothendieck, "Éléments de géométrie algébrique, III: Étude cohomologique des faisceaux cohérents I”, Inst. Hautes Études Sci. Publ. Math. 11 (1961), 167. MR 29 \#1209

[Grothendieck 1965] A. Grothendieck, "Éléments de géométrie algébrique, IV; Étude locale des schémas et des morphismes de schémas II", Inst. Hautes Études Sci. Publ. Math. 24 (1965), 231. MR 33 \#7330 Zbl 0135.39701

[Grothendieck 1967] A. Grothendieck, "Éléments de géométrie algébrique, IV: Étude locale des schémas et des morphismes de schémas IV", Inst. Hautes Études Sci. Publ. Math. 32 (1967), 361. MR 39 \#220 Zbl 0153.22301

[Hartshorne 1966] R. Hartshorne, Residues and duality, Lecture Notes in Mathematics 20, Springer, Berlin, 1966. MR 36 \#5145 Zbl 0212.26101

[Hartshorne 1977] R. Hartshorne, Algebraic geometry, Graduate Texts in Mathematics 52, Springer, New York, 1977. MR 57 \#3116 Zbl 0531.14001

[Iyengar and Krause 2006] S. Iyengar and H. Krause, "Acyclicity versus total acyclicity for complexes over Noetherian rings”, Doc. Math. 11 (2006), 207-240. MR 2007h:16013 Zbl 1119.13014

[Jørgensen 2005] P. Jørgensen, “The homotopy category of complexes of projective modules”, Adv. Math. 193:1 (2005), 223-232. MR 2005m:18013 Zbl 1068.18012

[Kaplansky 1958] I. Kaplansky, "Projective modules", Ann. of Math (2) 68 (1958), 372-377. MR 20 \#6453 Zbl 0083.25802

[Kapustin and Li 2003] A. Kapustin and Y. Li, "D-branes in Landau-Ginzburg models and algebraic geometry”, J. High Energy Phys. 12 (2003), 005, 44 pp. MR 2005b:81179b

[Krause 2005] H. Krause, "The stable derived category of a Noetherian scheme", Compos. Math. 141:5 (2005), 1128-1162. MR 2006e:18019 Zbl 1090.18006 
[Lin and Pomerleano 2013] K. H. Lin and D. Pomerleano, "Global matrix factorizations", Math. Res. Lett. 20:1 (2013), 91-106. MR 3126725 Zbl 1285.14019

[Lunts 2010] V. A. Lunts, "Categorical resolution of singularities", J. Algebra 323:10 (2010), 29773003. MR 2011d:18019 Zbl 1202.18006

[Murfet 2007] D. Murfet, The mock homotopy category of projectives and Grothendieck duality, Ph.D. thesis, Australian National University, 2007, available at http://www.therisingsea.org/thesis.pdf.

[Murfet 2013] D. Murfet, "Residues and duality for singularity categories of isolated Gorenstein singularities”, Compos. Math. 149:12 (2013), 2071-2100. MR 3143706 Zbl 06250163

[Neeman 1992] A. Neeman, "The connection between the $K$-theory localization theorem of Thomason, Trobaugh and Yao and the smashing subcategories of Bousfield and Ravenel", Ann. Sci. École Norm. Sup. (4) 25:5 (1992), 547-566. MR 93k:18015 Zbl 0868.19001

[Neeman 1996] A. Neeman, "The Grothendieck duality theorem via Bousfield's techniques and Brown representability”, J. Amer. Math. Soc. 9:1 (1996), 205-236. MR 96c:18006 Zbl 0864.14008

[Neeman 2001] A. Neeman, Triangulated categories, Annals of Mathematics Studies 148, Princeton University Press, 2001. MR 2001k:18010 Zbl 0974.18008

[Neeman 2008] A. Neeman, "The homotopy category of flat modules, and Grothendieck duality", Invent. Math. 174:2 (2008), 255-308. MR 2009h:16008 Zbl 1184.18008

[Orlov 2004] D. O. Orlov, "Triangulated categories of singularities and D-branes in Landau-Ginzburg models", Tr. Mat. Inst. Steklova 246 (2004), 240-262. In Russian; translated in Proc. Steklov Math. Inst. 246,(2004), 227-248. MR 2006i:81173 Zbl 1101.81093

[Orlov 2006] D. O. Orlov, "Triangulated categories of singularities, and equivalences between Landau-Ginzburg models”, Mat. Sb. 197:12 (2006), 1827-1840. MR 2009g:14013 Zbl 1161.14301

[Orlov 2011] D. Orlov, "Formal completions and idempotent completions of triangulated categories of singularities", Adv. Math. 226:1 (2011), 206-217. MR 2012c:14035 Zbl 1216.18012

[Orlov 2012] D. Orlov, "Matrix factorizations for nonaffine LG-models", Math. Ann. 353:1 (2012), 95-108. MR 2910782 Zbl 1243.81178

[Polishchuk and Positselski 2012] A. Polishchuk and L. Positselski, "Hochschild (co)homology of the second kind I”, Trans. Amer. Math. Soc. 364:10 (2012), 5311-5368. MR 2931331 Zbl 1285.16005

[Polishchuk and Vaintrob 2011] A. Polishchuk and A. Vaintrob, "Matrix factorizations and singularity categories for stacks", Ann. Inst. Fourier (Grenoble) 61:7 (2011), 2609-2642. MR 3112502 Zbl 1278.13014

[Polishchuk and Vaintrob 2014] A. Polishchuk and A. Vaintrob, "Matrix factorizations and cohomological field theories", Journ. für die reine und angewandte Math. (online publication April 2014).

[Positselski 2010] L. Positselski, Homological algebra of semimodules and semicontramodules, Instytut Matematyczny Polskiej Akademii Nauk. Monografie Matematyczne (New Series) 70, Birkhäuser/Springer Basel AG, 2010. MR 2012c:16030 Zbl 1202.18001

[Positselski 2011a] L. Positselski, "Mixed Artin-Tate motives with finite coefficients", Mosc. Math. J. 11:2 (2011), 317-402, 407-408. MR 2859239 Zbl 1273.12004

[Positselski 2011b] L. Positselski, "Two kinds of derived categories, Koszul duality, and comodulecontramodule correspondence", pp. vi+133 Mem. Amer. Math. Soc. 996, Amer. Math. Soc., Providence, RI, 2011. MR 2012f:16023

[Positselski 2012] L. Positselski, "Contraherent cosheaves", preprint, 2012. arXiv 1209.2995

[Positsel'skiǔ 1993] L. E. Positsel'skiǔ, "Nonhomogeneous quadratic duality and curvature”, Funktsional. Anal. i Prilozhen. 27:3 (1993), 57-66. In Russian; translated in Funct. Anal. Appl. 27:3 (1993), 197-283. MR 95h:16041 Zbl 0826.16041 
[Raynaud and Gruson 1971] M. Raynaud and L. Gruson, "Critères de platitude et de projectivité: Techniques de "platification" d'un module", Invent. Math. 13 (1971), 1-89. MR 46 \#7219 Zbl 0227.14010

[Thomason 1997] R. W. Thomason, "The classification of triangulated subcategories", Compositio Math. 105:1 (1997), 1-27. MR 98b:18017 Zbl 0873.18003

[Thomason and Trobaugh 1990] R. W. Thomason and T. Trobaugh, "Higher algebraic $K$-theory of schemes and of derived categories", pp. 247-435 in The Grothendieck Festschrift, Vol. III, edited by P. Cartier et al., Progr. Math. 88, Birkhäuser, Boston, 1990. MR 92f:19001 Zbl 0731.14001

Communicated by Michel Van den Bergh

Received 2014-12-26

Accepted 2015-03-27

efimov@mccme.ru

Department of Algebraic Geometry, Steklov Mathematical Institute of the Russian Academy of Sciences, Gubkina str., 8, Moscow, 119991, Russia, and Laboratory of Algebraic Geometry, Higher School of Economics, 7 Vavilova str., Moscow, 117312, Russia

posic@mccme.ru

Mathematics Department, Technion - Israel Institute of Technology, 32000 Haifa, Israel, and Sector of Algebra and Number Theory, Institute for Information Transmission Problems, Moscow, 127994, Russia, and Laboratory of Algebraic Geometry, National Research University Higher School of Economics, Moscow, 117312, Russia 


\section{Algebra \& Number Theory}

msp.org/ant

\section{EDITORS}

MANAGING EDITOR

Bjorn Poonen

Massachusetts Institute of Technology

Cambridge, USA

\author{
EDITORIAL BOARD CHAIR \\ David Eisenbud \\ University of California \\ Berkeley, USA
}

BOARD OF EDITORS

Georgia Benkart

Dave Benson

Richard E. Borcherds

John H. Coates

J-L. Colliot-Thélène

Brian D. Conrad

Hélène Esnault

Hubert Flenner

Sergey Fomin

Edward Frenkel

Andrew Granville

Joseph Gubeladze

Roger Heath-Brown

Craig Huneke

Kiran S. Kedlaya

János Kollár

Yuri Manin

Philippe Michel
University of Wisconsin, Madison, USA

University of Aberdeen, Scotland

University of California, Berkeley, USA

University of Cambridge, UK

CNRS, Université Paris-Sud, France

Stanford University, USA

Freie Universität Berlin, Germany

Ruhr-Universität, Germany

University of Michigan, USA

University of California, Berkeley, USA

Université de Montréal, Canada

San Francisco State University, USA

Oxford University, UK

University of Virginia, USA

Univ. of California, San Diego, USA

Princeton University, USA

Northwestern University, USA

École Polytechnique Fédérale de Lausanne
Susan Montgomery

Shigefumi Mori

Raman Parimala

Jonathan Pila

Anand Pillay

Victor Reiner

Peter Sarnak

Joseph H. Silverman

Michael Singer

Vasudevan Srinivas

J. Toby Stafford

Ravi Vakil

Michel van den Bergh

Marie-France Vignéras

Kei-Ichi Watanabe

Efim Zelmanov

Shou-Wu Zhang
University of Southern California, USA

RIMS, Kyoto University, Japan

Emory University, USA

University of Oxford, UK

University of Notre Dame, USA

University of Minnesota, USA

Princeton University, USA

Brown University, USA

North Carolina State University, USA

Tata Inst. of Fund. Research, India

University of Michigan, USA

Stanford University, USA

Hasselt University, Belgium

Université Paris VII, France

Nihon University, Japan

University of California, San Diego, USA

Princeton University, USA

\section{PRODUCTION}

production@msp.org

Silvio Levy, Scientific Editor

See inside back cover or msp.org/ant for submission instructions.

The subscription price for 2015 is US $\$ 255 /$ year for the electronic version, and $\$ 440 /$ year ( $+\$ 55$, if shipping outside the US) for print and electronic. Subscriptions, requests for back issues and changes of subscribers address should be sent to MSP.

Algebra \& Number Theory (ISSN 1944-7833 electronic, 1937-0652 printed) at Mathematical Sciences Publishers, 798 Evans Hall \#3840, c/o University of California, Berkeley, CA 94720-3840 is published continuously online. Periodical rate postage paid at Berkeley, CA 94704, and additional mailing offices.

ANT peer review and production are managed by EditFLOW ${ }^{\circledR}$ from MSP.

\section{PUBLISHED BY}

- mathematical sciences publishers

nonprofit scientific publishing

http://msp.org/

(C) 2015 Mathematical Sciences Publishers 


\section{Algebra \& Number Theory}

\section{Volume $9 \quad$ No. $5 \quad 2015$}

$p$-adic Hodge-theoretic properties of étale cohomology with mod $p$ coefficients, and the 1035 cohomology of Shimura varieties

MATTHEW EMERTON and Toby GeE

Homotopy exact sequences and orbifolds

KENTARO Mitsui

Factorially closed subrings of commutative rings

SAGNIK CHAKRABORTY, RAJENDRA VASANT GURJAR and MASAYOSHI MIYANISHI

Coherent analogues of matrix factorizations and relative singularity categories 